\title{
Structurally Diverse Synthesis of Five-, Six- and Seven-membered Benzosultams through Electrochemical Cyclization
}

Aiyun Liu, ${ }^{\text {a,b }}$ Tiantian Guo, ${ }^{\mathrm{a}, \mathrm{b}}$ Shuangshuang Zhang, ${ }^{\mathrm{a}, \mathrm{b}}$ Han Yang, ${ }^{\mathrm{a}}$ Qi Zhang, ${ }^{\text {a** }}$ Yonghai Chai ${ }^{\mathrm{a}, \mathrm{b} *}$ and Shengyong Zhang ${ }^{\mathrm{a}, \mathrm{b} *}$

a.Key Laboratory of Applied Surface and Colloid Chemistry, Ministry of Education, Xi'an, Shaanxi 710119, P. R. China.

${ }^{b}$ School of Chemistry and Chemical Engineering, Shaanxi Normal University, Xi'an, Shaanxi 710119, P. R. China.

\section{Table of Contents}

1. General information

2. Synthetic Procedures

2.1) Synthesis of $N$-alkyl-substituted benzene-sulfonamide derivatives

2.2) Synthesis of biarylsulfonamides

2.3) Synthesis of six and seven-membered benzoslutams

2.4) Synthesis of $\mathbf{2 u}$ from $2 \mathbf{s}$

2.5) Synthesis of 2-benzyl-benzenesulfonamide derivatives

2.6) Synthesis of five-membered benzoslutams

2.7) Large-scale synthesis of $7 \mathbf{a}$

4. X-Ray Crystal Structure and Data of $\mathbf{2 a}, \mathbf{2 q}$ and $\mathbf{2 r} \quad 46-50$

5 References 


\section{General information}

Dry acetonitrile $(\mathrm{MeCN})$ was obtained by refluxing with $\mathrm{CaH}_{2}$ under argon for more than $2 \mathrm{~h}$. Methanol (MeOH) is analytical grade, and hexafluoroisopropanol (HFIP) is commercially available. Analytical thin-layer chromatography (TLC) was carried out with plates of Silica GF254 (0.2 mm, Qingdao, China). Carbon rods are commercially available from Beijing Jing long Special Carbon Technology Co., Ltd. Platinum electrodes are commercially available from Wuhan GaoshiRuilian Technology Co., Ltd. Detection of compounds was achieved by UV absorption (254 nm). Column chromatography was performed using 300 mesh silica gel. Nuclear magnetic resonance spectra were afforded with Bruker Ascend ${ }^{\mathrm{TM}} 400 \mathrm{MHz}$ or $600 \mathrm{MHz}$ for ${ }^{1} \mathrm{H}$ NMR and $100 \mathrm{MHz}$ or $150 \mathrm{MHz}$ for ${ }^{13} \mathrm{C}$ NMR. Chemical shifts $(\delta)$ were reported in parts per million (ppm). Coupling constants $(J)$ were reported in Hertz. Multiplicity reported using the following abbreviations: s (singlet), $\mathrm{d}$ (doublet), $\mathrm{t}$ (triplet), $\mathrm{q}$ (quartet), $\mathrm{m}$ (multiplet), dd (doublet of doublet), dt (doublet of triplet), td (triplet of doublet). High resolution mass spectra were acquired by ESI ionization sources using Bruker mass spectrometer Maxis, X-ray data was obtained from Bruker D8 VENTURE, ${ }^{1} \mathrm{H}$ NMR spectra were referenced to $7.26\left(\mathrm{CDCl}_{3}\right), 2.05\left(\mathrm{CD}_{3} \mathrm{COCD}_{3}-\mathrm{d}_{6}\right), 2.50$ (DMSO-d $\left.\mathrm{d}_{6}\right) .{ }^{13} \mathrm{C}$ NMR spectra were referenced to $77.00\left(\mathrm{CDCl}_{3}\right), 30.20\left(\mathrm{CD}_{3} \mathrm{COCD}_{3}-\mathrm{d}_{6}\right), 39.50$ (DMSO$\left.\mathrm{d}_{6}\right)$.
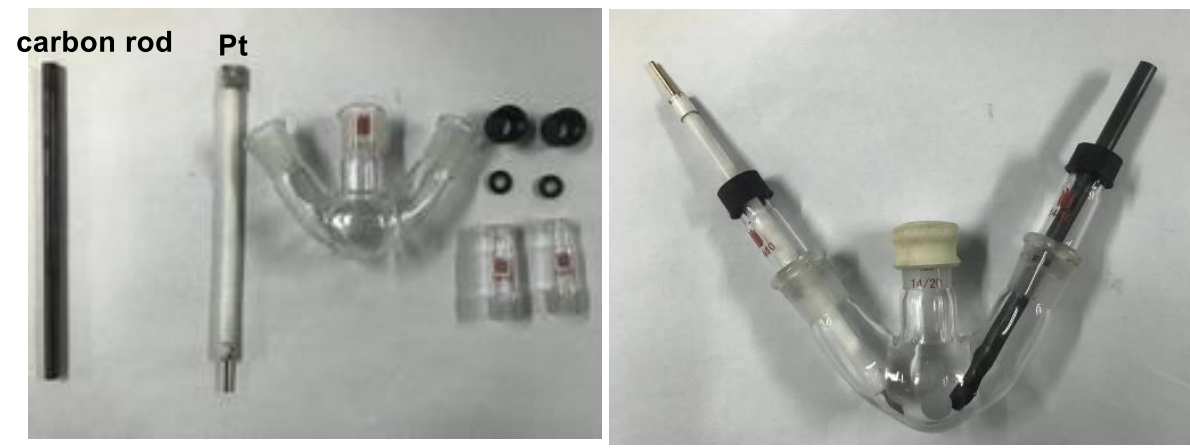

Figure S1. Electrolysis setup 


\section{Synthetic Procedures}

\section{1) Synthesis of $N$-alkyl-substituted benzene-sulfonamide derivatives ${ }^{1}$}

\section{General procedure:}

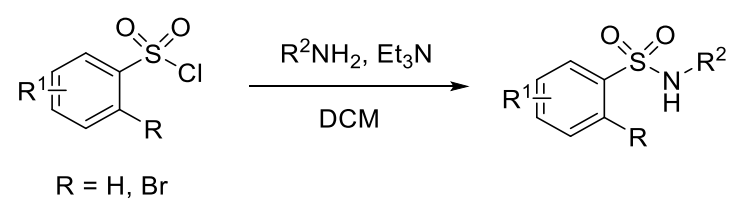

Primary amine (18 mmol,1.2 equiv) and $\mathrm{Et}_{3} \mathrm{~N}$ ( $30 \mathrm{mmol}, 2$ equiv) were added to round bottom flask, then benzenesulfonyl chloride (15 mmol, 1 equiv) in dichloromethane was injected into it at $0{ }^{\circ} \mathrm{C}$. After the mixture was stirred until TLC showed that benzenesulfonyl chloride was totally consumed at room temperature. Water was added to the reaction mixture and extracted with dichloromethane $(3 \mathrm{x})$. The combined organic layers were dried over $\mathrm{Na}_{2} \mathrm{SO}_{4}$, concentrated under reduced pressure. The residue was purified by column chromatography (ethyl acetate : petroleum ether v/v) on silica gel or recrystallized from ethyl acetate/petroleum ether to give the corresponding products. 2-bromo- $N$-methylbenzenesulfonamide $(\mathrm{S} 1)^{2}$<smiles>CNS(=O)(=O)c1ccccc1Br</smiles>

White solid; $3.34 \mathrm{~g}, 89 \%$ yield; m.p. $112.5-113.2{ }^{\circ} \mathrm{C}$; the product is purified by crystallization from EtOAc/ petroleum ether (1:6, $14 \mathrm{~mL}) ;{ }^{1} \mathrm{H} \mathrm{NMR}\left(400 \mathrm{MHz}, \mathrm{CDCl}_{3}\right)$ $\delta 8.15(\mathrm{~d}, J=7.6 \mathrm{~Hz}, 1 \mathrm{H}), 7.74(\mathrm{~d}, J=7.6 \mathrm{~Hz}, 1 \mathrm{H}), 7.48(\mathrm{t}, J=7.6 \mathrm{~Hz}, 1 \mathrm{H}), 7.42(\mathrm{t}, J$ $=7.6 \mathrm{~Hz}, 1 \mathrm{H}), 5.06(\mathrm{~s}, 1 \mathrm{H}), 2.62(\mathrm{~d}, J=4.8 \mathrm{~Hz}, 3 \mathrm{H}) ;{ }^{13} \mathrm{C} \mathrm{NMR}\left(100 \mathrm{MHz}, \mathrm{CDCl}_{3}\right) \delta$ 137.7, 135.0, 133.8, 132.1, 127.8, 119.6, 29.2; HRMS (ESI-TOF) m/z: [M+Na $]^{+}$Calcd for $\mathrm{C}_{7} \mathrm{H}_{8} \mathrm{BrNO}_{2} \mathrm{SNa} 271.9351$; Found 271.9360.

2-bromo- $N$-(1-methylethyl)benzenesulfonamide (S2)<smiles>CC(C)NS(=O)(=O)c1ccccc1</smiles>

White solid; $3.62 \mathrm{~g}, 87 \%$ yield; m.p. $100-100.7{ }^{\circ} \mathrm{C}$; the product is purified by crystallization from EtOAc/ petroleum ether (1:6, $14 \mathrm{~mL}) ;{ }^{1} \mathrm{H} \mathrm{NMR}\left(600 \mathrm{MHz}, \mathrm{CDCl}_{3}\right)$ $\delta 8.16(\mathrm{dd}, J=7.8,1.8 \mathrm{~Hz}, 1 \mathrm{H}), 7.73(\mathrm{dd}, J=7.8,1.2 \mathrm{~Hz}, 1 \mathrm{H}), 7.47(\mathrm{td}, J=7.8,1.2 \mathrm{~Hz}$, 
1H), $7.41(\mathrm{td}, J=7.8,1.8 \mathrm{~Hz}, 1 \mathrm{H}), 4.98(\mathrm{~d}, J=7.2 \mathrm{~Hz}, 1 \mathrm{H}), 3.46-3.39$ (m, 1H), 1.09 $(\mathrm{d}, J=6.6 \mathrm{~Hz}, 6 \mathrm{H}) ;{ }^{13} \mathrm{C} \mathrm{NMR}\left(150 \mathrm{MHz}, \mathrm{CDCl}_{3}\right) \delta 139.9,135.0,133.5,131.4,127.8$, 119.7, 46.6, 23.4; HRMS (ESI-TOF) m/z: $[\mathrm{M}+\mathrm{Na}]^{+}$Calcd for $\mathrm{C}_{9} \mathrm{H}_{12} \mathrm{BrNO}_{2} \mathrm{SNa}$ 299.9664; Found 299.9673.

2-bromo- $N$-(1,1-dimethylethyl)benzenesulfonamide $(\mathrm{S} 3)^{3}$<smiles>CC(C)(C)NS(=O)(=O)c1ccccc1Br</smiles>

White solid; $2.76 \mathrm{~g}, 63 \%$ yield; m.p. $164-164.5^{\circ} \mathrm{C}$; the product is purified by crystallization from EtOAc/ petroleum ether (1:6, $14 \mathrm{~mL}) ;{ }^{1} \mathrm{H} \mathrm{NMR}\left(400 \mathrm{MHz}, \mathrm{CDCl}_{3}\right)$ $\delta 8.17(\mathrm{~d}, J=7.6 \mathrm{~Hz}, 1 \mathrm{H}), 7.71(\mathrm{~d}, J=7.6 \mathrm{~Hz}, 1 \mathrm{H}), 7.45(\mathrm{t}, J=7.6 \mathrm{~Hz}, 1 \mathrm{H}), 7.38(\mathrm{t}, J=$ $7.6 \mathrm{~Hz}, 1 \mathrm{H}), 5.08(\mathrm{~s}, 1 \mathrm{H}), 1.22(\mathrm{~s}, 9 \mathrm{H}) ;{ }^{13} \mathrm{C} \mathrm{NMR}\left(150 \mathrm{MHz}, \mathrm{CDCl}_{3}\right) \delta 142.3,134.9$, 133.1, 130.7, 127.9, 119.7, 54.9, 30.0; HRMS (ESI-TOF) $\mathrm{m} / \mathrm{z}:[\mathrm{M}+\mathrm{Na}]^{+}$calcd for $\mathrm{C}_{10} \mathrm{H}_{14} \mathrm{BrNO}_{2} \mathrm{SNa}$ 313.9821; Found 313.9831.

$\mathrm{N}$-methylbenzenesulfonamide (S4)<smiles>CNS(=O)(=O)c1ccccc1</smiles>

Oil; $2.44 \mathrm{~g}, 95 \%$ yield; the product was obtained using EtOAc / petroleum ether (1:6) as the eluent; ${ }^{1} \mathrm{H} \mathrm{NMR}\left(600 \mathrm{MHz}, \mathrm{CDCl}_{3}\right) \delta 7.87(\mathrm{~d}, J=7.8 \mathrm{~Hz}, 2 \mathrm{H}), 7.60(\mathrm{t}, J=7.8$ $\mathrm{Hz}, 1 \mathrm{H}), 7.53(\mathrm{t}, J=7.8 \mathrm{~Hz}, 2 \mathrm{H}), 4.41(\mathrm{brs}, 1 \mathrm{H}), 2.67(\mathrm{~d}, J=5.4 \mathrm{~Hz}, 3 \mathrm{H}) ;{ }^{13} \mathrm{C} \mathrm{NMR}$ $\left(150 \mathrm{MHz}, \mathrm{CDCl}_{3}\right) \delta 138.8,132.7,129.1,127.2,29.4 ; \mathrm{HRMS}(\mathrm{ESI}-\mathrm{TOF}) \mathrm{m} / \mathrm{z}:[\mathrm{M}+\mathrm{Na}]^{+}$ Calcd for $\mathrm{C}_{7} \mathrm{H}_{9} \mathrm{NO}_{2} \mathrm{SNa}$ 194.0246; Found 194.0251.

$\mathrm{N}$-(1-methylethyl)benzenesulfonamide (S5) ${ }^{5}$

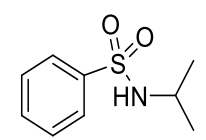

Oil; $2.93 \mathrm{~g}, 98 \%$ yield; the product was obtained using EtOAc / petroleum ether (1:6) as the eluent; ${ }^{1} \mathrm{H}$ NMR $\left(400 \mathrm{MHz}, \mathrm{CDCl}_{3}\right) \delta 7.91-7.88(\mathrm{~m}, 2 \mathrm{H}), 7.59-7.54(\mathrm{~m}, 1 \mathrm{H})$, $7.53-7.48(\mathrm{~m}, 2 \mathrm{H}), 4.57$ (brs, 1H), $3.53-3.41(\mathrm{~m}, 1 \mathrm{H}), 1.07$ (d, $J=6.4 \mathrm{~Hz}, 6 \mathrm{H})$; ${ }^{13} \mathrm{C}$ NMR $\left(100 \mathrm{MHz}, \mathrm{CDCl}_{3}\right) \delta 141.1,132.4,129.0,126.9,46.1,23.7$; HRMS (ESITOF) m/z: $[\mathrm{M}+\mathrm{Na}]^{+}$Calcd for $\mathrm{C}_{9} \mathrm{H}_{13} \mathrm{NO}_{2} \mathrm{SNa} 222.0559$; Found 222.0563. 


\section{$N$-(1,1-dimethylethyl)benzenesulfonamide (S6)}

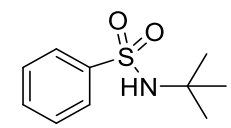

White solid; $3.07 \mathrm{~g}, 96 \%$ yield; m.p. $76.2-77.0{ }^{\circ} \mathrm{C}$; the product was obtained using EtOAc / petroleum ether (1:6) as the eluent; ${ }^{1} \mathrm{H}$ NMR (400 MHz, $\left.\mathrm{CDCl}_{3}\right) \delta 7.92-7.89$ (m, 2H), $7.56-7.52(\mathrm{~m}, 1 \mathrm{H}), 7.51-7.46(\mathrm{~m}, 2 \mathrm{H}), 4.72$ (brs, $1 \mathrm{H}), 1.22(\mathrm{~s}, 9 \mathrm{H}) ;{ }^{13} \mathrm{C}$ NMR (100 MHz, $\left.\mathrm{CDCl}_{3}\right) \delta 143.4,132.1,128.8,126.9,54.7,30.1$; HRMS (ESI-TOF) m/z: $[\mathrm{M}+\mathrm{Na}]^{+}$Calcd for $\mathrm{C}_{10} \mathrm{H}_{15} \mathrm{NO}_{2} \mathrm{SNa} 236.0716$; Found 236.0719.

\section{4-methyl- $N$-(1-methylethyl)benzenesulfonamide (S7) ${ }^{6}$}

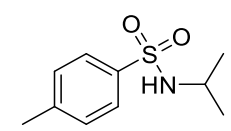

Oil; $2.91 \mathrm{~g}, 91 \%$ yield; the product was obtained using EtOAc / petroleum ether (1:6,) as the eluent; ${ }^{1} \mathrm{H}$ NMR $\left(600 \mathrm{MHz}, \mathrm{CDCl}_{3}\right) \delta 7.76(\mathrm{~d}, J=8.2 \mathrm{~Hz}, 1 \mathrm{H}), 7.29(\mathrm{~d}, J=8.2$ $\mathrm{Hz}, 1 \mathrm{H}), 4.50(\mathrm{~d}, J=7.2 \mathrm{~Hz}, 1 \mathrm{H}), 3.48-3.40(\mathrm{~m}, 1 \mathrm{H}), 2.42(\mathrm{~s}, 3 \mathrm{H}), 1.07(\mathrm{~d}, J=6.6 \mathrm{~Hz}$, $6 \mathrm{H}) ;{ }^{13} \mathrm{C} \mathrm{NMR}\left(150 \mathrm{MHz}, \mathrm{CDCl}_{3}\right) \delta 143.2,138.1,129.6,127.0,46.0,23.7,21.5 . \mathrm{HRMS}$ (ESI-TOF) m/z: $[\mathrm{M}+\mathrm{Na}]^{+}$Calcd for $\mathrm{C}_{10} \mathrm{H}_{15} \mathrm{NO}_{2} \mathrm{SNa} 236.0716$; Found 236.0720. 3-methyl- $N$-(1-methylethyl)benzenesulfonamide (S8)<smiles>Cc1ccc(S(=O)(=O)NC(C)C)cc1I</smiles>

White solid; $2.27 \mathrm{~g}, 71 \%$ yield; m.p. $53.2-53.8{ }^{\circ} \mathrm{C}$; the product was obtained using EtOAc / petroleum ether (1:6) as the eluent; ${ }^{1} \mathrm{H}$ NMR (400 MHz, $\left.\mathrm{CDCl}_{3}\right) \delta 7.69-7.66$ (m, 2H), $7.41-7.35(\mathrm{~m}, 2 \mathrm{H}), 4.36$ (brs, 1H), $3.50-3.45(\mathrm{~m}, 1 \mathrm{H}), 2.42(\mathrm{~s}, 3 \mathrm{H}), 1.08(\mathrm{~d}$, $J=6.6 \mathrm{~Hz}, 6 \mathrm{H}) ;{ }^{13} \mathrm{C} \mathrm{NMR}\left(100 \mathrm{MHz}, \mathrm{CDCl}_{3}\right) \delta 140.9,139.2,133.2,128.9,127.3$, 124.1, 46.1, 23.8, 21.3. HRMS (ESI-TOF) m/z: $[\mathrm{M}+\mathrm{Na}]^{+}$Calcd for $\mathrm{C}_{10} \mathrm{H}_{15} \mathrm{NO}_{2} \mathrm{SNa}$ 236.0716; Found 236.0725.

\section{2-chloro- $N$-(1-methylethyl)benzenesulfonamide (S9)}<smiles>CC(C)NS(=O)(=O)c1ccccc1Cl</smiles>

White solid; $2.87 \mathrm{~g}, 82 \%$ yield; m.p. $91.2-91.9{ }^{\circ} \mathrm{C}$; the product is purified by 
crystallization from EtOAc/ petroleum ether (1:6, $14 \mathrm{~mL}) ;{ }^{1} \mathrm{H} \mathrm{NMR}\left(600 \mathrm{MHz}, \mathrm{CDCl}_{3}\right)$ $\delta 8.12(\mathrm{dd}, J=7.8,1.2 \mathrm{~Hz}, 1 \mathrm{H}), 7.53-7.40(\mathrm{~m}, 2 \mathrm{H}), 7.42-7.40(\mathrm{~m}, 1 \mathrm{H}), 4.85(\mathrm{~d}, J=$ $6.6 \mathrm{~Hz}, 1 \mathrm{H}), 3.48-3.40(\mathrm{~m}, 1 \mathrm{H}), 1.09(\mathrm{~d}, J=6.6 \mathrm{~Hz}, 6 \mathrm{H}) ;{ }^{13} \mathrm{C} \mathrm{NMR}\left(100 \mathrm{MHz}, \mathrm{CDCl}_{3}\right)$ $\delta 138.2,133.5,131.5,131.3,131.0,127.2,46.5,23.4$. HRMS (ESI-TOF) m/z: $[\mathrm{M}+\mathrm{Na}]^{+}$ Calcd for $\mathrm{C}_{9} \mathrm{H}_{12} \mathrm{ClNO}_{2} \mathrm{SNa} 256.0169$; Found 256.0180.

\section{3-chloro-N-(1-methylethyl)benzenesulfonamide (S10)}<smiles>CC(C)NS(=O)(=O)c1cccc(Cl)c1</smiles>

White solid; $2.98 \mathrm{~g}, 85 \%$ yield; m.p. $37.1-38.2{ }^{\circ} \mathrm{C}$; the product is purified by crystallization from EtOAc/ petroleum ether $(1: 6,14 \mathrm{~mL}) ;{ }^{1} \mathrm{H} \mathrm{NMR}\left(600 \mathrm{MHz}, \mathrm{CDCl}_{3}\right)$ $\delta 7.88-7.87(\mathrm{~m}, 1 \mathrm{H}), 7.77-7.76(\mathrm{~m}, 1 \mathrm{H}), 7.55-7.53(\mathrm{~m}, 1 \mathrm{H}), 7.45(\mathrm{t}, J=7.8 \mathrm{~Hz}, 1 \mathrm{H})$, $4.45(\mathrm{~d}, J=7.8 \mathrm{~Hz}, 1 \mathrm{H}), 3.54-3.47(\mathrm{~m}, 1 \mathrm{H}), 1.05(\mathrm{~d}, J=6.6 \mathrm{~Hz}, 6 \mathrm{H}) ;{ }^{13} \mathrm{C}$ NMR $(150$ $\left.\mathrm{MHz}, \mathrm{CDCl}_{3}\right) \delta 143.0,135.2,132.6,130.4,127.1,125.0,46.3,23.7 ;$ HRMS (ESI-TOF) $\mathrm{m} / \mathrm{z}:[\mathrm{M}+\mathrm{Na}]^{+}$Calcd for $\mathrm{C}_{9} \mathrm{H}_{12} \mathrm{ClNO}_{2} \mathrm{SNa} 256.0169$; Found 256.0177.

\section{4-chloro- $N$-(1-methylethyl)benzenesulfonamide $(\mathrm{S} 11)^{7}$}

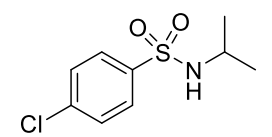

White solid; $2.94 \mathrm{~g}, 84 \%$ yield; m.p. $83.2-83.7{ }^{\circ} \mathrm{C}$; the product is purified by crystallization from EtOAc/ petroleum ether (1:6, $14 \mathrm{~mL}) ;{ }^{1} \mathrm{H} \mathrm{NMR}\left(600 \mathrm{MHz}, \mathrm{CDCl}_{3}\right)$ $\delta 7.82(\mathrm{~d}, J=8.4 \mathrm{~Hz}, 2 \mathrm{H}), 7.48(\mathrm{~d}, J=8.4 \mathrm{~Hz}, 2 \mathrm{H}), 4.77(\mathrm{~d}, J=7.8 \mathrm{~Hz}, 1 \mathrm{H}), 3.50-$ $3.43(\mathrm{~m}, 1 \mathrm{H}), 1.08(\mathrm{~d}, J=6.6 \mathrm{~Hz}, 6 \mathrm{H}) ;{ }^{13} \mathrm{C} \mathrm{NMR}\left(150 \mathrm{MHz}, \mathrm{CDCl}_{3}\right) \delta=139.7,138.9$, 129.3, 128.4, 46.2, 23.7; HRMS (ESI-TOF) m/z: $[\mathrm{M}+\mathrm{Na}]^{+}$Calcd for $\mathrm{C}_{9} \mathrm{H}_{12} \mathrm{ClNO}_{2} \mathrm{SNa}$ 256.0169; Found 256.0178. 


\section{2) Synthesis of biarylsulfonamides ${ }^{8}$}

\section{General procedure:}

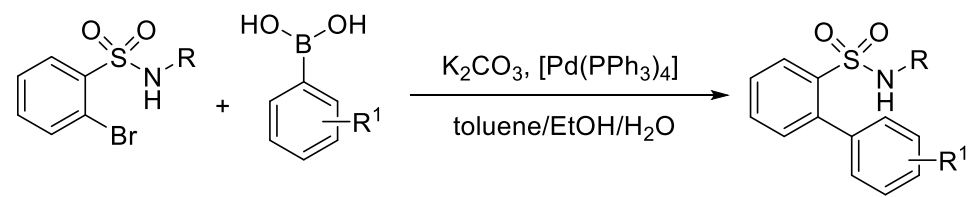

The bromide ( $2.0 \mathrm{mmol}, 1.0$ equiv), the corresponding boronic acid (3.0 mmol, 1.5 equiv) and $\mathrm{K}_{2} \mathrm{CO}_{3}$ (8.0 mmol, 4.0 equiv) are suspended in a mixture of toluene, $\mathrm{EtOH}$ and $\mathrm{H}_{2} \mathrm{O}(0.1 \mathrm{M}, 20 \mathrm{~mL}, 5: 2: 1, \mathrm{v} / \mathrm{v} / \mathrm{v})$ and heated to reflux in oil bath for $30 \mathrm{~min}$. Under argon, $\left[\mathrm{Pd}\left(\mathrm{PPh}_{3}\right)_{4}\right](58 \mathrm{mg}, 2.5 \mathrm{~mol} \%)$ is added and the resulting dark suspension is heated to reflux. Upon completion, the mixture is cooled, $\mathrm{H}_{2} \mathrm{O}$ and EtOAc $(1: 1,30 \mathrm{~mL})$ are added and the phases separated. The aqueous phases are extracted with EtOAc. The combined organic phase was washed with brine, dried over $\mathrm{Na}_{2} \mathrm{SO}_{4}$, filtered and concentrated, purified by flash silica gel chromatography (EtOAc/petroleum ether v/v). $N$-isopropyl-[1,1'-biphenyl]-2-sulfonamide (1a)<smiles>CC(C)NS(=O)(=O)c1ccccc1-c1ccccc1</smiles>

White solid; 0.53 g, $96 \%$ yield; m.p. $67.5-68.1{ }^{\circ} \mathrm{C}$, eluent: EA/PE $=1: 10 ;{ }^{1} \mathrm{H}$ NMR $(400$ $\left.\mathrm{MHz}, \mathrm{CDCl}_{3}\right) \delta 8.17(\mathrm{dd}, J=8.0,1.2 \mathrm{~Hz}, 1 \mathrm{H}), 7.59(\mathrm{td}, J=7.6,1.6 \mathrm{~Hz}, 1 \mathrm{H}), 7.52-$ $7.43(\mathrm{~m}, 6 \mathrm{H}), 7.34(\mathrm{dd}, J=7.6,1.2 \mathrm{~Hz}, 1 \mathrm{H}), 3.41(\mathrm{~d}, J=6.0 \mathrm{~Hz}, 1 \mathrm{H}), 3.32-3.20(\mathrm{~m}$, 1H), $0.89(\mathrm{~d}, J=6.4 \mathrm{~Hz}, 6 \mathrm{H}) ;{ }^{13} \mathrm{C} \mathrm{NMR}\left(150 \mathrm{MHz}, \mathrm{CDCl}_{3}\right) \delta 140.2,139.7,139.0,132.4$, 132.0, 129.9, 128.9, 128.4, 128.0, 127.7, 46.2, 23.3; HRMS (ESI) m/z: [M+Na] $]^{+}$Calcd for $\mathrm{C}_{15} \mathrm{H}_{17} \mathrm{NO}_{2} \mathrm{SNa} 298.0872$; Found 298.0873.

\section{$N$-(tert-butyl)-[1,1'-biphenyl]-2-sulfonamide (1b)}<smiles>CN(C(=O)c1ccccc1-c1ccccc1S(=O)(=O)O)C(C)(C)C</smiles>

White solid; 0.53 g, $92 \%$ yield; m.p. $121.4-121.8{ }^{\circ} \mathrm{C}$, eluent: $\mathrm{EA} / \mathrm{PE}=1: 10 ;{ }^{1} \mathrm{H}$ NMR $\left(400 \mathrm{MHz}, \mathrm{CDCl}_{3}\right) \delta 8.18(\mathrm{dd}, J=8.0,1.2 \mathrm{~Hz}, 1 \mathrm{H}), 7.58-7.52(\mathrm{~m}, 3 \mathrm{H}), 7.50-7.41(\mathrm{~m}$, 4H), $7.32(\mathrm{~d}, J=7.6 \mathrm{~Hz}, 1 \mathrm{H}), 3.50(\mathrm{~s}, 1 \mathrm{H}), 0.99(\mathrm{~s}, 9 \mathrm{H}) ;{ }^{13} \mathrm{C} \mathrm{NMR}\left(100 \mathrm{MHz}, \mathrm{CDCl}_{3}\right)$ 
$\delta 142.2,139.9,139.5,132.2,131.7,129.9,128.3,128.2,128.1,127.8,54.3,29.7 ;$ HRMS

(ESI-TOF) m/z: [M+Na] ${ }^{+}$Calcd for $\mathrm{C}_{16} \mathrm{H}_{19} \mathrm{NO}_{2} \mathrm{SNa} 312.1029$; Found 312.1020 .

$N$-methyl-[1, 1'-biphenyl]-2-sulfonamide (1c) ${ }^{8}$

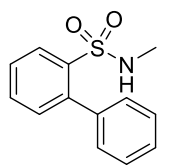

White solid; 0.43 g, $87 \%$ yield; m.p. $119.0-119.3{ }^{\circ} \mathrm{C}$, eluent: EA/PE $=1: 10 ;{ }^{1} \mathrm{H}$ NMR $\left(400 \mathrm{MHz}, \mathrm{CDCl}_{3}\right) \delta 8.16(\mathrm{~d}, J=7.6 \mathrm{~Hz}, 1 \mathrm{H}), 7.61(\mathrm{t}, J=7.6 \mathrm{~Hz}, 1 \mathrm{H}), 7.53(\mathrm{t}, J=7.6$ Hz, 1H), $7.50-7.45$ (m, 5H), 7.34 (d, $J=7.6 \mathrm{~Hz}, 1 \mathrm{H}), 3.31$ (brs, 1H), 2.34 (d, $J=5.2$ $\mathrm{Hz}, 3 \mathrm{H}) ;{ }^{13} \mathrm{C} \mathrm{NMR}\left(150 \mathrm{MHz}, \mathrm{CDCl}_{3}\right) \delta 140.1,138.8,136.9,132.3,132.0,129.8,129.0$, 128.6, 128.3, 128.0, 29.0; HRMS (ESI-TOF) m/z: [M+Na $]^{+}$Calcd for $\mathrm{C}_{13} \mathrm{H}_{13} \mathrm{NO}_{2} \mathrm{SNa}$ 270.0559; Found 270.0559.

4'-(tert-butyl)- $N$-isopropyl-[1,1'-biphenyl]-2-sulfonamide (1d)

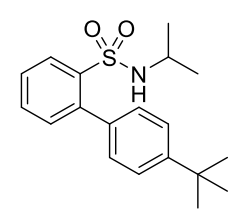

White solid; 0.63 g, 95\% yield; m.p. $111.1-111.7{ }^{\circ} \mathrm{C}$, eluent: $\mathrm{EA} / \mathrm{PE}=1: 10 ;{ }^{1} \mathrm{H}$ NMR $\left(400 \mathrm{MHz}, \mathrm{CDCl}_{3}\right) \delta 8.16(\mathrm{dd}, J=8.0,1.2 \mathrm{~Hz}, 1 \mathrm{H}), 7.57(\mathrm{td}, J=7.6,1.2 \mathrm{~Hz}, 1 \mathrm{H}), 7.50$ $-7.44(\mathrm{~m}, 5 \mathrm{H}), 7.35(\mathrm{dd}, J=7.6,1.2 \mathrm{~Hz}, 1 \mathrm{H}), 3.40(\mathrm{~d}, J=6.0 \mathrm{~Hz}, 1 \mathrm{H}), 3.29-3.16(\mathrm{~m}$, 1H), 1.37 (s, 9H), $0.85(\mathrm{~d}, J=6.8 \mathrm{~Hz}, 6 \mathrm{H}) ;{ }^{13} \mathrm{C} \mathrm{NMR}\left(100 \mathrm{MHz}, \mathrm{CDCl}_{3}\right) \delta 151.6,140.1$, 139.7, 135.9, 132.5, 132.0, 129.6, 128.8, 127.6, 125.0, 46.1, 34.7, 31.3, 23.1; HRMS (ESI-TOF) m/z: [M+Na] ${ }^{+}$Calcd for $\mathrm{C}_{19} \mathrm{H}_{25} \mathrm{NO}_{2} \mathrm{SNa}$ 354.1498; Found 354.1500.

\section{$N$-isopropyl-4'-methyl-[1,1'-biphenyl]-2-sulfonamide (1e)}

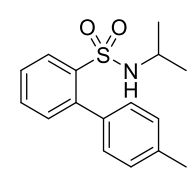

White solid; 0.57 g, $98 \%$ yield; m.p. $72.7-73.2{ }^{\circ} \mathrm{C}$, eluent: $\mathrm{EA} / \mathrm{PE}=1: 10 ;{ }^{1} \mathrm{H}$ NMR $(600$ MHz, Acetone-d 6$) \delta 8.10(\mathrm{dd}, J=7.8,1.2 \mathrm{~Hz}, 1 \mathrm{H}), 7.66(\mathrm{td}, J=7.2,1.2 \mathrm{~Hz}, 1 \mathrm{H}), 7.56$ $(\mathrm{td}, J=7.8,1.2 \mathrm{~Hz}, 1 \mathrm{H}), 7.36-7.34(\mathrm{~m}, 3 \mathrm{H}), 7.24-7.23(\mathrm{~m}, 2 \mathrm{H}), 5.01(\mathrm{~d}, J=6.0 \mathrm{~Hz}$, 1H), $3.26-3.18(\mathrm{~m}, 1 \mathrm{H}), 2.39(\mathrm{~s}, 3 \mathrm{H}), 0.95(\mathrm{~d}, J=6.6 \mathrm{~Hz}, 6 \mathrm{H}) ;{ }^{13} \mathrm{C} \mathrm{NMR}(150 \mathrm{MHz}$, $\left.\mathrm{CDCl}_{3}\right) \delta 140.3,139.6,138.3,136.0,132.5,132.0,129.8,128.9,128.7,127.5,46.2$, 
23.3, 21.2; HRMS (ESI-TOF) m/z: $[\mathrm{M}+\mathrm{Na}]^{+}$Calcd for $\mathrm{C}_{16} \mathrm{H}_{19} \mathrm{NO}_{2} \mathrm{SNa} 312.1029$; Found 312.1027.

\section{$N$-isopropyl-4'-propyl-[1,1'-biphenyl]-2-sulfonamide (1f)}

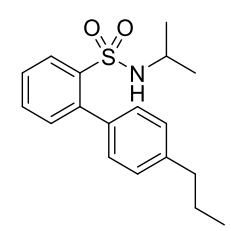

White solid; 0.60 g, $95 \%$ yield; m.p. 80.0-80.5 ${ }^{\circ} \mathrm{C}$, eluent: EA/PE $=1: 10 ;{ }^{1} \mathrm{H}$ NMR $(600$ MHz, Acetone-d $\left.\mathrm{d}_{6}\right) \delta 8.11(\mathrm{dd}, J=7.8,1.2 \mathrm{~Hz}, 1 \mathrm{H}), 7.66(\mathrm{td}, J=7.2,1.2 \mathrm{~Hz}, 1 \mathrm{H}), 7.57$ $(\mathrm{td}, J=7.8,1.2 \mathrm{~Hz}, 1 \mathrm{H}), 7.40-7.39$ (m, 2H), 7.37 (dd, $J=7.8,1.2 \mathrm{~Hz}, 1 \mathrm{H}), 7.27-7.26$ $(\mathrm{m}, 2 \mathrm{H}), 4.91(\mathrm{~d}, J=6.6 \mathrm{~Hz}, 1 \mathrm{H}), 3.23-3.15(\mathrm{~m}, 1 \mathrm{H}), 2.66(\mathrm{t}, J=7.8 \mathrm{~Hz} 2 \mathrm{H}), 1.72-$ $1.66(\mathrm{~m}, 2 \mathrm{H}), 0.96(\mathrm{t}, J=7.2 \mathrm{~Hz}, 3 \mathrm{H}), 0.94(\mathrm{~d}, J=6.6 \mathrm{~Hz}, 6 \mathrm{H}) ;{ }^{13} \mathrm{C} \mathrm{NMR}(100 \mathrm{MHz}$, $\left.\mathrm{CDCl}_{3}\right) \delta 143.1,140.2,139.7,136.2,132.5,132.0,129.8,128.8,128.2,127.5,46.1$, 37.7, 24.4, 23.2, 13.8; HRMS (ESI-TOF) m/z: $[\mathrm{M}+\mathrm{Na}]^{+}$Calcd for $\mathrm{C}_{18} \mathrm{H}_{23} \mathrm{NO}_{2} \mathrm{SNa}$ 340.1342; Found 340.1346.

\section{$N$-isopropyl-4' -methoxy-[1,1'-biphenyl]-2-sulfonamide (1g)}

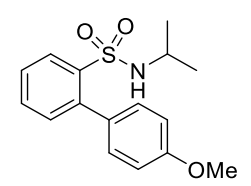

White solid; 0.57 g, $93 \%$ yield; m.p. $109.1-109.7{ }^{\circ} \mathrm{C}$, eluent: $\mathrm{EA} / \mathrm{PE}=1: 10 ;{ }^{1} \mathrm{H}$ NMR $\left(400 \mathrm{MHz}, \mathrm{CDCl}_{3}\right) \delta 8.16(\mathrm{dd}, J=8.0,1.2 \mathrm{~Hz}, 1 \mathrm{H}), 7.57(\mathrm{td}, J=7.6,1.2 \mathrm{~Hz}, 1 \mathrm{H}), 7.48$ $(\mathrm{dd}, J=7.6,1.2 \mathrm{~Hz}, 1 \mathrm{H}), 7.47-7.42(\mathrm{~m}, 2 \mathrm{H}), 7.32(\mathrm{dd}, J=7.6,1.2 \mathrm{~Hz}, 1 \mathrm{H}), 7.00-$ $6.94(\mathrm{~m}, 2 \mathrm{H}), 3.87(\mathrm{~s}, 3 \mathrm{H}), 3.49(\mathrm{~d}, J=6.4 \mathrm{~Hz}, 1 \mathrm{H}), 3.30-3.19(\mathrm{~m}, 1 \mathrm{H}), 0.89$ (d, $J=$ $6.8 \mathrm{~Hz}, 6 \mathrm{H}) ;{ }^{13} \mathrm{C} \mathrm{NMR}\left(100 \mathrm{MHz}, \mathrm{CDCl}_{3}\right) \delta 159.6,140.0,139.7,132.7,132.0,131.2$, 131.0, 128.9, 127.4, 113.4, 55.3, 46.2, 23.3; HRMS (ESI-TOF) m/z: [M+Na ${ }^{+}$Calcd for $\mathrm{C}_{16} \mathrm{H}_{19} \mathrm{NO}_{3} \mathrm{SNa} 328.0978$; Found 328.0976.

\section{$N$-isopropyl-[1,1': 4',1"'-terphenyl]-2-sulfonamide (1h)}<smiles>CC(C)NS(=O)(=O)c1ccccc1-c1ccc(-c2ccccc2)cc1</smiles> 
White solid; 0.58 g, $82 \%$ yield; m.p. 81.6-82.2 ${ }^{\circ} \mathrm{C}$, eluent: EA/PE $=1: 10 ;{ }^{1} \mathrm{H}$ NMR $(600$ $\left.\mathrm{MHz}, \mathrm{CDCl}_{3}\right) \delta 8.19(\mathrm{dd}, J=8.0,1.2 \mathrm{~Hz}, 1 \mathrm{H}), 7.71-7.69(\mathrm{~m}, 2 \mathrm{H}), 7.67-7.66(\mathrm{~m}, 2 \mathrm{H})$, $7.62-7.58(\mathrm{~m}, 3 \mathrm{H}), 7.52(\mathrm{td}, J=7.8,1.2 \mathrm{~Hz}, 1 \mathrm{H}), 7.50-7.47(\mathrm{~m}, 2 \mathrm{H}), 7.41-7.38(\mathrm{~m}$, $2 \mathrm{H}), 3.54(\mathrm{~d}, J=6.6 \mathrm{~Hz}, 1 \mathrm{H}), 3.32-3.25(\mathrm{~m}, 1 \mathrm{H}), 0.91(\mathrm{~d}, J=6.6 \mathrm{~Hz}, 6 \mathrm{H}) ;{ }^{13} \mathrm{C} \mathrm{NMR}$ $\left(150 \mathrm{MHz}, \mathrm{CDCl}_{3}\right) \delta 141.1,140.2,139.9,139.6,137.9,132.5,132.1,130.4,129.0$, 128.9, 127.8, 127.7, 127.1, 126.6, 46.2, 23.3; HRMS (ESI-TOF) m/z: [M+Na] $]^{+}$Calcd for $\mathrm{C}_{21} \mathrm{H}_{21} \mathrm{NO}_{2} \mathrm{SNa}$ 374.1185; Found 374.1182.

\section{4'-chloro- $N$-isopropyl-[1,1'-biphenyl]-2-sulfonamide (1i)}

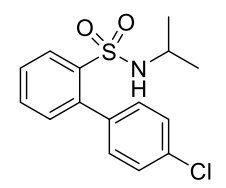

White solid; 0.58 g, $94 \%$ yield; m.p. $93.8-94.4{ }^{\circ} \mathrm{C}$, eluent: $\mathrm{EA} / \mathrm{PE}=1: 10 ;{ }^{1} \mathrm{H}$ NMR $(400$ $\left.\mathrm{MHz}, \mathrm{CDCl}_{3}\right) \delta 8.15(\mathrm{dd}, J=8.0,1.2 \mathrm{~Hz}, 1 \mathrm{H}), 7.59(\mathrm{td}, J=7.6,1.2 \mathrm{~Hz}, 1 \mathrm{H}), 7.51(\mathrm{td}$, $J=7.6,1.2 \mathrm{~Hz}, 1 \mathrm{H}), 7.44-7.40(\mathrm{~m}, 4 \mathrm{H}), 7.29(\mathrm{dd}, J=7.6,1.2 \mathrm{~Hz}, 1 \mathrm{H}), 3.54(\mathrm{~d}, J=6.8$ $\mathrm{Hz}, 1 \mathrm{H}), 3.31-3.26(\mathrm{~m}, 1 \mathrm{H}), 0.93(\mathrm{~d}, J=6.8 \mathrm{~Hz}, 6 \mathrm{H}) ;{ }^{13} \mathrm{C} \mathrm{NMR}\left(100 \mathrm{MHz}, \mathrm{CDCl}_{3}\right) \delta$ 139.6, 139.2, 137.5, 134.5, 132.4, 132.2, 131.2, 129.1, 128.1, 128.0, 46.2, 23.4; HRMS (ESI-TOF) m/z: [M+Na] ${ }^{+}$Calcd for $\mathrm{C}_{15} \mathrm{H}_{16} \mathrm{ClNO}_{2} \mathrm{SNa} 332.0482$; Found 332.0486.

\section{4'-fluoro- $N$-isopropyl-[1,1'-biphenyl]-2-sulfonamide (1j)}

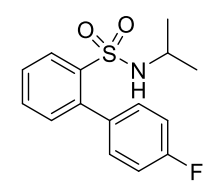

White solid; 0.56 g, $95 \%$ yield; m.p. $83.5-84{ }^{\circ} \mathrm{C}$, eluent: EA/PE $=1: 10 ;{ }^{1} \mathrm{H}$ NMR $(400$ $\left.\mathrm{MHz}, \mathrm{CDCl}_{3}\right) \delta 8.16(\mathrm{dd}, J=7.8,1.2 \mathrm{~Hz}, 1 \mathrm{H}), 7.58(\mathrm{td}, J=7.6,1.2 \mathrm{~Hz}, 1 \mathrm{H}), 7.51(\mathrm{dd}$, $J=7.8,1.2 \mathrm{~Hz}, 1 \mathrm{H}), 7.49-7.45(\mathrm{~m}, 2 \mathrm{H}), 7.31(\mathrm{dd}, J=7.6,1.2 \mathrm{~Hz}, 1 \mathrm{H}), 7.17-7.11(\mathrm{~m}$, $2 \mathrm{H}), 3.46(\mathrm{~d}, J=6.8 \mathrm{~Hz}, 1 \mathrm{H}), 3.34-3.23(\mathrm{~m}, 1 \mathrm{H}), 0.92(\mathrm{~d}, J=6.4 \mathrm{~Hz}, 6 \mathrm{H}) ;{ }^{13} \mathrm{C} \mathrm{NMR}$ $\left(150 \mathrm{MHz}, \mathrm{CDCl}_{3}\right) \delta 162.6(\mathrm{~d}, J=246.8 \mathrm{~Hz}), 139.7,139.4,134.9(\mathrm{~d}, J=3.2 \mathrm{~Hz}), 132.6$, $131.9(\mathrm{~d}, J=8.1 \mathrm{~Hz}), 131.7,129.0,127.9,115.0(\mathrm{~d}, J=21.3 \mathrm{~Hz}), 46.2,23.4 ;{ }^{19} \mathrm{~F}$ NMR

$\left(375 \mathrm{MHz}, \mathrm{CDCl}_{3}\right) \quad \delta$-113.2; $\mathrm{HRMS}$ (ESI-TOF) $\mathrm{m} / \mathrm{z}:[\mathrm{M}+\mathrm{Na}]^{+}$Calcd for $\mathrm{C}_{15} \mathrm{H}_{16} \mathrm{FNO}_{2} \mathrm{SNa}$ 316.0778; Found 316.0775. 


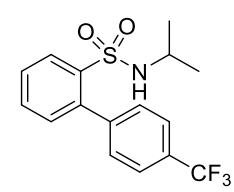

White solid; 0.49 g, $71 \%$ yield, m.p. $75.7-76.4{ }^{\circ} \mathrm{C}$, eluent: EA/PE $=1: 12 ;{ }^{1} \mathrm{H}$ NMR $(400$ $\left.\mathrm{MHz}, \mathrm{CDCl}_{3}\right) \delta 8.17(\mathrm{dd}, J=8.0,1.2 \mathrm{~Hz}, 1 \mathrm{H}), 7.72-7.70(\mathrm{~m}, 2 \mathrm{H}), 7.64-7.60(\mathrm{~m}, 3 \mathrm{H})$, $7.54(\mathrm{td}, J=7.6,1.2 \mathrm{~Hz}, 1 \mathrm{H}), 7.31(\mathrm{dd}, J=7.6,1.2 \mathrm{~Hz}, 1 \mathrm{H}), 3.50(\mathrm{~d}, J=7.2 \mathrm{~Hz}, 1 \mathrm{H})$, $3.37-3.26(\mathrm{~m}, 1 \mathrm{H}), 0.94(\mathrm{~d}, J=6.8 \mathrm{~Hz}, 6 \mathrm{H}) ;{ }^{13} \mathrm{C} \mathrm{NMR}\left(100 \mathrm{MHz}, \mathrm{CDCl}_{3}\right) \delta$ 142.9, (q, $J=1.2 \mathrm{~Hz}), 139.6,139.1,132.3,132.2,130.5130 .3,130.2,129.1,128.3,125.3,124.8(\mathrm{q}$, $J=3.8 \mathrm{~Hz}), 122.6,46.2,23.4 ;{ }^{19} \mathrm{~F}$ NMR $\left(375 \mathrm{MHz}, \mathrm{CDCl}_{3}\right) \delta$-62.6; HRMS (ESI-TOF) $\mathrm{m} / \mathrm{z}:[\mathrm{M}+\mathrm{Na}]^{+}$Calcd for $\mathrm{C}_{16} \mathrm{H}_{16} \mathrm{~F}_{3} \mathrm{NO}_{2} \mathrm{SNa}$ 366.0746; Found 366.0752.

\section{4'-chloro- $N$-methyl-[1,1'-biphenyl]-2-sulfonamide $(11)^{8}$}

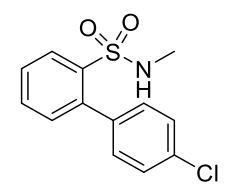

White solid; $0.41 \mathrm{~g}, 72 \%$ yield; m.p. $129.6-131.0{ }^{\circ} \mathrm{C}$, eluent: $\mathrm{EA} / \mathrm{PE}=1: 10 ;{ }^{1} \mathrm{H}$ NMR $\left(600 \mathrm{MHz}, \mathrm{CDCl}_{3}\right) \delta 8.15(\mathrm{dd}, J=8.0,1.2 \mathrm{~Hz}, 1 \mathrm{H}), 7.61(\mathrm{td}, J=7.8,1.2 \mathrm{~Hz}, 1 \mathrm{H}), 7.54$ $(\mathrm{td}, J=7.8,1.2 \mathrm{~Hz}, 1 \mathrm{H}), 7.44-7.41(\mathrm{~m}, 4 \mathrm{H}), 7.31(\mathrm{dd}, J=7.8,1.2 \mathrm{~Hz}, 1 \mathrm{H}), 3.44(\mathrm{q}, J$ $=5.4 \mathrm{~Hz}, 1 \mathrm{H}), 2.38(\mathrm{~d}, J=5.4 \mathrm{~Hz}, 3 \mathrm{H}) ;{ }^{13} \mathrm{C} \mathrm{NMR}\left(150 \mathrm{MHz}, \mathrm{CDCl}_{3}\right) \delta 139.1,137.3$, 137.0, 134.7, 132.4, 132.1, 130.4, 129.9, 128.4, 128.3, 28.9; HRMS (APCI-TOF) m/z: Calcd for $\mathrm{C}_{13} \mathrm{H}_{13} \mathrm{ClNO}_{2} \mathrm{~S}[\mathrm{M}+\mathrm{H}]^{+}$282.0350; Found 282.0347.

\section{$N$-isopropyl-3', 5'-dimethyl-[1,1'-biphenyl]-2-sulfonamide (1m)}

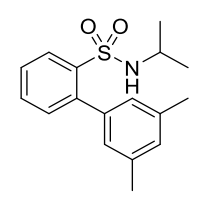

White solid; 0.52 g, $86 \%$ yield; m.p. $65.3-66{ }^{\circ} \mathrm{C}$, eluent: EA/PE $=1: 10 ;{ }^{1} \mathrm{H}$ NMR $(400$ $\left.\mathrm{MHz}, \mathrm{CDCl}_{3}\right) \delta 8.15(\mathrm{dd}, J=8.0,1.2 \mathrm{~Hz}, 1 \mathrm{H}), 7.56(\mathrm{td}, J=7.6,1.2 \mathrm{~Hz}, 1 \mathrm{H}), 7.47(\mathrm{td}$, $J=7.6,1.2 \mathrm{~Hz}, 1 \mathrm{H}), 7.32(\mathrm{dd}, J=7.6,1.2 \mathrm{~Hz}, 1 \mathrm{H}), 7.10(\mathrm{~s}, 2 \mathrm{H}), 7.07(\mathrm{~s}, 1 \mathrm{H}), 3.47(\mathrm{~d}$, $J=5.6 \mathrm{~Hz}, 1 \mathrm{H}), 3.29-3.18(\mathrm{~m}, 1 \mathrm{H}), 2.37(\mathrm{~s}, 6 \mathrm{H}), 0.90(\mathrm{~d}, J=6.4 \mathrm{~Hz}, 6 \mathrm{H}) ;{ }^{13} \mathrm{C} \mathrm{NMR}$ $\left(100 \mathrm{MHz}, \mathrm{CDCl}_{3}\right) \delta 140.4,139.5,138.8,137.0,132.2,131.9,129.9,128.8,127.7$, 
127.5, 46.2, 23.2, 21.3; HRMS (ESI-TOF) $\mathrm{m} / \mathrm{z}$ : $[\mathrm{M}+\mathrm{Na}]^{+}$Calcd for $\mathrm{C}_{17} \mathrm{H}_{21} \mathrm{NO}_{2} \mathrm{SNa}$ 326.1185; Found 326.1193.

\section{$N$-isopropyl-3'-methyl-[1,1'-biphenyl]-2-sulfonamide (1n)}

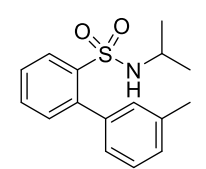

Oil; 0.56 g, $96 \%$ yield, eluent: EA/PE $=1: 10 ;{ }^{1} \mathrm{H}$ NMR $(600 \mathrm{MHz} \text {, Acetone-d })_{6} \delta 8.11$ $(\mathrm{dd}, J=8.4,1.2 \mathrm{~Hz}, 1 \mathrm{H}), 7.66(\mathrm{td}, J=7.8,1.2 \mathrm{~Hz}, 1 \mathrm{H}), 7.57(\mathrm{td}, J=7.8,1.2 \mathrm{~Hz}, 1 \mathrm{H})$, $7.35(\mathrm{dd}, J=7.8,1.2 \mathrm{~Hz}, 1 \mathrm{H}), 7.31-7.22(\mathrm{~m}, 4 \mathrm{H}), 5.02(\mathrm{~d}, J=6.0 \mathrm{~Hz}, 1 \mathrm{H}), 3.25-3.18$ $(\mathrm{m}, 1 \mathrm{H}), 2.37(\mathrm{~s}, 3 \mathrm{H}), 0.96(\mathrm{~d}, J=6.6 \mathrm{~Hz}, 6 \mathrm{H}) ;{ }^{13} \mathrm{C} \mathrm{NMR}\left(100 \mathrm{MHz}, \mathrm{CDCl}_{3}\right) \delta 140.3$, 139.6, 138.8, 137.7, 132.3, 132.0, 130.6, 129.0, 128.8, 127.9, 127.6, 126.9, 46.2, 23.2, 21.4; HRMS (ESI-TOF) m/z: [M+Na] ${ }^{+}$Calcd for $\mathrm{C}_{16} \mathrm{H}_{19} \mathrm{NO}_{2} \mathrm{SNa}$ 312.1029; Found 312.1033 .

\section{3'-fluoro- $N$-isopropyl-[1,1'-biphenyl]-2-sulfonamide (10)}

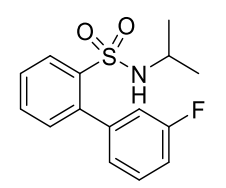

White solid; 0.56 g, $96 \%$ yield; m.p. $74.0-74.6{ }^{\circ} \mathrm{C}$, eluent: $\mathrm{EA} / \mathrm{PE}=1: 10 ;{ }^{1} \mathrm{H}$ NMR $(600$ $\left.\mathrm{MHz}, \mathrm{CDCl}_{3}\right) \delta 8.16(\mathrm{dd}, J=8.4,1.2 \mathrm{~Hz}, 1 \mathrm{H}), 7.59(\mathrm{td}, J=7.8,1.2 \mathrm{~Hz}, 1 \mathrm{H}), 7.52(\mathrm{td}$, $J=7.8,1.2 \mathrm{~Hz}, 1 \mathrm{H}), 7.42(\mathrm{td}, J=7.8,6.0 \mathrm{~Hz}, 1 \mathrm{H}), 7.32(\mathrm{dd}, J=7.8,1.2 \mathrm{~Hz}, 1 \mathrm{H}), 7.28$ $(\mathrm{dt}, J=7.8,1.2 \mathrm{~Hz}, 1 \mathrm{H}), 7.22-7.20(\mathrm{~m}, 1 \mathrm{H}), 7.15-7.12(\mathrm{~m}, 1 \mathrm{H}), 3.51(\mathrm{~d}, J=6.0 \mathrm{~Hz}$, $1 \mathrm{H}), 3.34-3.27(\mathrm{~m}, 1 \mathrm{H}), 0.94(\mathrm{~d}, J=6.6 \mathrm{~Hz}, 6 \mathrm{H}) ;{ }^{13} \mathrm{C} \mathrm{NMR}\left(100 \mathrm{MHz}, \mathrm{CDCl}_{3}\right) \delta$ $162.0(J=245.8 \mathrm{~Hz}), 141.2,141.1,139.3(J=2.0 \mathrm{~Hz}), 132.2(J=14.3 \mathrm{~Hz}), 129.5(J=$ 8.2 Hz), 129.0, 128.1, $125.6(J=3.1 \mathrm{~Hz}), 117.2,117.0,115.2(J=20.8 \mathrm{~Hz}), 46.2,23.4$; ${ }^{19} \mathrm{~F}$ NMR $\left(375 \mathrm{MHz}, \mathrm{CDCl}_{3}\right) \delta-112.7$; HRMS (ESI-TOF) $\mathrm{m} / \mathrm{z}$ : $[\mathrm{M}+\mathrm{Na}]^{+}$Calcd for $\mathrm{C}_{15} \mathrm{H}_{16} \mathrm{FNO}_{2} \mathrm{SNa}$ 316.0778; Found 316.0782.

\section{2'-fluoro- $N$-isopropyl-[1,1'-biphenyl]-2-sulfonamide (1p)}

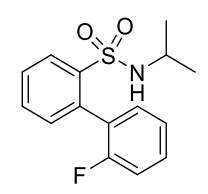


White solid; 0.55 g, $93 \%$ yield; m.p. 97.2-98. $1{ }^{\circ} \mathrm{C}$, eluent: $\mathrm{EA} / \mathrm{PE}=1: 10 ;{ }^{1} \mathrm{H}$ NMR $(600$ $\left.\mathrm{MHz}, \mathrm{CDCl}_{3}\right) \delta 8.17(\mathrm{dd}, J=8.0,1.2 \mathrm{~Hz}, 1 \mathrm{H}), 7.60(\mathrm{td}, J=7.8,1.2 \mathrm{~Hz}, 1 \mathrm{H}), 7.54(\mathrm{td}$, $J=7.8,1.2 \mathrm{~Hz}, 1 \mathrm{H}), 7.46-7.40(\mathrm{~m}, 2 \mathrm{H}), 7.35(\mathrm{~d}, J=7.8 \mathrm{~Hz}, 1 \mathrm{H}), 7.22(\mathrm{td}, J=7.8,1.2$ $\mathrm{Hz}, 1 \mathrm{H}), 7.18-7.15(\mathrm{~m}, 1 \mathrm{H}), 3.62(\mathrm{~d}, J=6.6 \mathrm{~Hz}, 1 \mathrm{H}), 3.42-3.34(\mathrm{~m}, 1 \mathrm{H}), 1.06$ (d, $J$ $=6.6 \mathrm{~Hz}, 3 \mathrm{H}), 0.93(\mathrm{~d}, J=6.6 \mathrm{~Hz}, 3 \mathrm{H}) ;{ }^{13} \mathrm{C} \mathrm{NMR}\left(100 \mathrm{MHz}, \mathrm{CDCl}_{3}\right) \delta 159.2(J=244.4$ $\mathrm{Hz}), 140.2,133.9,132.8(J=0.8 \mathrm{~Hz}), 132.3(J=2.6 \mathrm{~Hz}), 131.9,130.4(J=8.0 \mathrm{~Hz})$, 129.2, 128.4, $126.6(J=15.9 \mathrm{~Hz}), 123.6(J=3.6 \mathrm{~Hz}), 115.4(J=21.9 \mathrm{~Hz}), 46.2,23.8$, 23.0; ${ }^{19} \mathrm{~F}$ NMR $\left(375 \mathrm{MHz}, \mathrm{CDCl}_{3}\right) \delta-112.8$; HRMS (ESI-TOF) m/z: $[\mathrm{M}+\mathrm{Na}]^{+} \mathrm{Calcd}$ for $\mathrm{C}_{15} \mathrm{H}_{16} \mathrm{FNO}_{2} \mathrm{SNa} 316.0778$; Found 316.0785.

\section{$N$-isopropyl-2'-methyl-[1,1'-biphenyl]-2-sulfonamide (1q)}

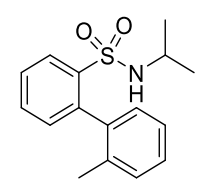

White solid; 0.49 g, $85 \%$ yield; m.p. 54.6-55.4 ${ }^{\circ} \mathrm{C}$, eluent: EA/PE $=1: 10 ;{ }^{1} \mathrm{H}$ NMR $(600$ $\left.\mathrm{MHz}, \mathrm{CDCl}_{3}\right) \delta 8.15(\mathrm{dd}, J=8.0,1.2 \mathrm{~Hz}, 1 \mathrm{H}), 7.58(\mathrm{td}, J=7.2,1.2 \mathrm{~Hz}, 1 \mathrm{H}), 7.50(\mathrm{td}$, $J=7.8,1.2 \mathrm{~Hz}, 1 \mathrm{H}), 7.34-7.29(\mathrm{~m}, 2 \mathrm{H}), 7.25-7.22(\mathrm{~m}, 2 \mathrm{H}), 7.18(\mathrm{dd}, J=7.8,1.2 \mathrm{~Hz}$ $1 \mathrm{H}), 3.58(\mathrm{~d}, J=6.0 \mathrm{~Hz}, 1 \mathrm{H}), 3.44-3.36(\mathrm{~m}, 1 \mathrm{H}), 2.10(\mathrm{~s}, 3 \mathrm{H}), 1.08(\mathrm{~d}, J=6.6 \mathrm{~Hz}$, $3 \mathrm{H}), 0.98(\mathrm{~d}, J=6.6 \mathrm{~Hz}, 3 \mathrm{H}) ;{ }^{13} \mathrm{C} \mathrm{NMR}\left(100 \mathrm{MHz}, \mathrm{CDCl}_{3}\right) \delta 140.0,139.7,138.5,137.6$, 132.1, 132.0, 130.3, 128.9, 128.8, 128.5, 127.6, 124.8, 46.2, 23.9, 23.3, 20.5.; HRMS (ESI-TOF) m/z: [M+Na] ${ }^{+}$Calcd for $\mathrm{C}_{16} \mathrm{H}_{19} \mathrm{NO}_{2} \mathrm{SNa} 312.1029$; found 312.1037.

\section{2'-ethyl- $N$-isopropyl-[1,1'-biphenyl]-2-sulfonamide (1r)}

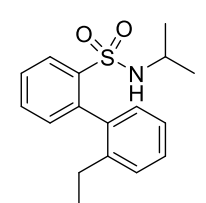

White solid; 0.54 g, $89 \%$ yield; m.p. $45.4-46.3{ }^{\circ} \mathrm{C}$, eluent: EA/PE $=1: 10 ;{ }^{1} \mathrm{H}$ NMR $(400$ MHz, Acetone-d 6 ) $\delta 8.10(\mathrm{dd}, J=8.0,1.6 \mathrm{~Hz}, 1 \mathrm{H}), 7.66(\mathrm{td}, J=7.6,1.6 \mathrm{~Hz}, 1 \mathrm{H}), 7.59$ $(\mathrm{td}, J=7.6,1.6 \mathrm{~Hz}, 1 \mathrm{H}), 7.35-7.27(\mathrm{~m}, 3 \mathrm{H}), 7.21-7.14(\mathrm{~m}, 2 \mathrm{H}), 5.32(\mathrm{~d}, \mathrm{~J}=7.2 \mathrm{~Hz}$, 1H), $3.39-3.28(\mathrm{~m}, 1 \mathrm{H}), 2.49-2.26(\mathrm{~m}, 2 \mathrm{H}), 1.09(\mathrm{~d}, J=6.4 \mathrm{~Hz}, 3 \mathrm{H}), 1.06(\mathrm{t}, J=7.6$ $\mathrm{Hz}, 3 \mathrm{H}), 1.02(\mathrm{~d}, J=6.4 \mathrm{~Hz}, 3 \mathrm{H}) ;{ }^{13} \mathrm{C} \mathrm{NMR}\left(100 \mathrm{MHz}, \mathrm{CDCl}_{3}\right) \delta 143.6,139.9,139.7$, 
$138.0,132.2,131.8,129.0,128.73,128.72,128.4,127.7,124.6,46.2,26.5,23.9,23.3$, 14.7; HRMS (ESI-TOF) m/z: [M+Na $]^{+}$Calcd for $\mathrm{C}_{17} \mathrm{H}_{21} \mathrm{NO}_{2} \mathrm{SNa} 326.1185$; Found 326.1189 .

\section{$N$-(tert-butyl)-2'-methyl-[1,1'-biphenyl]-2-sulfonamide (1s)}

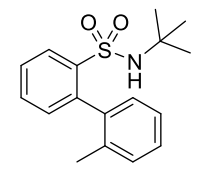

White solid; $0.50 \mathrm{~g}, 82 \%$ yield, eluent: $\mathrm{EA} / \mathrm{PE}=1: 10 ;{ }^{1} \mathrm{H} \mathrm{NMR}\left(600 \mathrm{MHz}, \mathrm{CDCl}_{3}\right) \delta$ $8.17(\mathrm{dd}, J=8.0,1.2 \mathrm{~Hz}, 1 \mathrm{H}), 7.56(\mathrm{td}, J=7.2,1.2 \mathrm{~Hz}, 1 \mathrm{H}), 7.48(\mathrm{td}, J=7.8,1.2 \mathrm{~Hz}$, 1H), $7.33-7.29(\mathrm{~m}, 2 \mathrm{H}), 7.24-7.21(\mathrm{~m}, 3 \mathrm{H}), 3.64(\mathrm{~s}, 1 \mathrm{H}), 2.11(\mathrm{~s}, 3 \mathrm{H}), 1.16(\mathrm{~s}, 9 \mathrm{H})$; ${ }^{13} \mathrm{C}$ NMR $\left(150 \mathrm{MHz}, \mathrm{CDCl}_{3}\right) \delta 142.0,139.6,138.5,137.5,131.8,131.7,130.3,129.6$, 128.4, 128.2, 127.6, 124.7, 54.7, 30.1, 20.5; HRMS (ESI-TOF) m/z: $[\mathrm{M}+\mathrm{Na}]^{+} \mathrm{Calcd}$ for $\mathrm{C}_{17} \mathrm{H}_{21} \mathrm{NO}_{2} \mathrm{SNa} 326.1185$; Found 326.1188.

\section{$N$-(tert-butyl)-2'-ethyl-[1,1'-biphenyl]-2-sulfonamide (1t)}

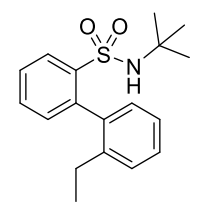

White solid; $0.5 \mathrm{~g}, 78 \%$ yield, eluent: $\mathrm{EA} / \mathrm{PE}=1: 10 ;{ }^{1} \mathrm{H} \mathrm{NMR}\left(600 \mathrm{MHz}, \mathrm{CDCl}_{3}\right) \delta$ $8.16(\mathrm{dd}, J=8.0,1.2 \mathrm{~Hz}, 1 \mathrm{H}), 7.55(\mathrm{td}, J=7.8,1.2 \mathrm{~Hz}, 1 \mathrm{H}), 7.48(\mathrm{td}, J=7.8,1.2 \mathrm{~Hz}$, 1H), $7.39-7.35(\mathrm{~m}, 2 \mathrm{H}), 7.25-7.21(\mathrm{~m}, 3 \mathrm{H}), 3.63(\mathrm{~s}, 1 \mathrm{H}), 2.49(\mathrm{dq}, J=15.0,7.8 \mathrm{~Hz}$, 1H), $2.32(\mathrm{dq}, J=15.0,7.8 \mathrm{~Hz}, 1 \mathrm{H}), 1.16(\mathrm{~s}, 9 \mathrm{H}), 1.10(\mathrm{t}, J=7.8 \mathrm{~Hz}, 3 \mathrm{H}) ;{ }^{13} \mathrm{C}$ NMR $\left(150 \mathrm{MHz}, \mathrm{CDCl}_{3}\right) \delta 143.6,142.0,139.5,137.9,132.0,131.5,129.6,128.7,128.4$, 128.1, 127.7, 124.5, 54.7, 30.1, 26.6, 14.9; HRMS (ESI-TOF) m/z: $[\mathrm{M}+\mathrm{Na}]^{+}$Calcd for $\mathrm{C}_{18} \mathrm{H}_{23} \mathrm{NO}_{2} \mathrm{SNa} 340.1342$; Found 340.1339. 


\section{3) Synthesis of six and seven-membered benzoslutams}

\section{General procedure:}

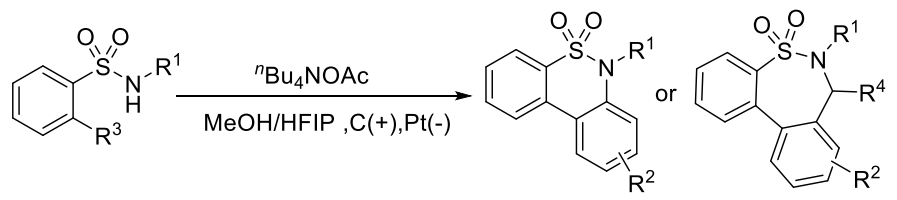

Substrate (0.2 mmol), $n$-Bu 4 NOAc $(0.2 \mathrm{mmol}, 60.3 \mathrm{mg})$ and a mixture of $\mathrm{MeOH} / \mathrm{HFIP}$ (V/V $4.8 \mathrm{~mL} / 1.2 \mathrm{~mL}$ ) were added to a $10 \mathrm{~mL}$ three-necked round-bottomed flask equipped with carbon rod $(6 \mathrm{~mm})$ as the anode and platinum plate $(1 \mathrm{~cm} \times 1 \mathrm{~cm} \times 0.2$ $\mathrm{mm})$ as the cathode. Under the argon atmosphere, the reaction mixture was heated in oil bath and electrolyzed at a constant current of $15 \mathrm{~mA}$ for $2.5 \mathrm{~h}$ at $60{ }^{\circ} \mathrm{C}$. Upon completion, the reaction mixture was concentrated under reduced pressure. The residue was chromatographed by silica gel chromatography (ethyl acetate/ petroleum ether V/V) to give the desired products.

\section{6-isopropyl-6H-dibenzo[c,e][1,2] thiazine 5,5-dioxide (2a) ${ }^{9}$}<smiles>CC(C)N1c2ccccc2-c2ccccc2S1(=O)=O</smiles>

White solid; $44 \mathrm{mg}, 81 \%$ yield; m.p. $127.9-128.8^{\circ} \mathrm{C}$, eluent: $\mathrm{EA} / \mathrm{PE}=1: 12$ to $1: 10 ;{ }^{1} \mathrm{H}$ NMR $\left(600 \mathrm{MHz}, \mathrm{CDCl}_{3}\right) \delta 7.96-7.95(\mathrm{~m}, 2 \mathrm{H}), 7.91(\mathrm{~d}, J=7.8 \mathrm{~Hz}, 1 \mathrm{H}), 7.68(\mathrm{td}, J=$ 7.8, 1.2 Hz, 1H), 7.55 (td, $J=7.2,1.2 \mathrm{~Hz}, 1 \mathrm{H}), 7.46-7.42$ (m, 3H), 4.44 (hept, $J=6.8$ $\mathrm{Hz}, 1 \mathrm{H}), 1.09(\mathrm{~d}, J=6.8 \mathrm{~Hz}, 6 \mathrm{H}) ;{ }^{13} \mathrm{C} \mathrm{NMR}\left(150 \mathrm{MHz}, \mathrm{CDCl}_{3}\right) \delta 137.2,136.6,133.2$, 132.4, 129.6, 128.7, 128.4 127.3, 127.2, 125.7, 125.5, 123.0, 54.9, 21.6; HRMS (ESITOF) $\mathrm{m} / \mathrm{z}:[\mathrm{M}+\mathrm{Na}]^{+}$Calcd for $\mathrm{C}_{15} \mathrm{H}_{15} \mathrm{NO}_{2} \mathrm{SNa}$ 296.0716; Found 296.0710.

\section{6-(tert-butyl)-6H-dibenzo[c,e][1,2]thiazine 5,5-dioxide (2b)}<smiles>CC(C)(C)N1c2ccccc2-c2ccccc2S1(=O)=O</smiles>

White solid; $45 \mathrm{mg}$, $78 \%$ yield; m.p. $157.5-158.5{ }^{\circ} \mathrm{C}$, eluent: $\mathrm{EA} / \mathrm{PE}=1: 12$ to $1: 10 ;{ }^{1} \mathrm{H}$ NMR (600 MHz, $\left.\mathrm{CDCl}_{3}\right) \delta 7.94(\mathrm{dd}, J=7.8,1.2 \mathrm{~Hz}, 1 \mathrm{H}), 7.87(\mathrm{~d}, J=7.8 \mathrm{~Hz}, 1 \mathrm{H}), 7.84$ $(\mathrm{dd}, J=7.8,1.2 \mathrm{~Hz}, 1 \mathrm{H}), 7.66(\mathrm{td}, J=7.8,1.2 \mathrm{~Hz}, 1 \mathrm{H}), 7.53(\mathrm{td}, J=7.8,1.2 \mathrm{~Hz}, 1 \mathrm{H})$, 
$7.44-7.36$ (m, 3H), 1.24 (s, 9H); ${ }^{13} \mathrm{C}$ NMR (100 MHz, $\left.\mathrm{CDCl}_{3}\right) \delta$ 138.3, 133.9, 132.3, 131.4, 129.8, 128.7, 128.6, 127.9, 125.8, 125.2, 123.5, 63.4, 30.6; HRMS (ESI-TOF) m/z: $[\mathrm{M}+\mathrm{Na}]^{+}$Calcd for $\mathrm{C}_{16} \mathrm{H}_{17} \mathrm{NO}_{2} \mathrm{SNa} 310.0872$; Found 310.0877 .

\section{6-methyl-6H-dibenzo[c,e $][1,2]$ thiazine 5,5 -dioxide $(2 \mathrm{c})^{8}$}<smiles>CN1c2ccccc2-c2ccccc2S1(=O)=O</smiles>

White solid; $33 \mathrm{mg}, 68 \%$ yield; m.p. $100.6-101.6{ }^{\circ} \mathrm{C}$, eluent: $\mathrm{EA} / \mathrm{PE}=1: 12$ to $1: 10 ;{ }^{1} \mathrm{H}$ NMR (600 MHz, $\left.\mathrm{CDCl}_{3}\right) \delta 8.02-8.01(\mathrm{~m}, 2 \mathrm{H}), 7.97(\mathrm{~d}, J=7.8 \mathrm{~Hz}, 1 \mathrm{H}), 7.71(\mathrm{td}, J=$ 7.2,1.2 Hz, 1H), $7.57(\mathrm{td}, J=7.8,1.2 \mathrm{~Hz}, 1 \mathrm{H}), 7.51(\mathrm{td}, J=7.8,1.2 \mathrm{~Hz}, 1 \mathrm{H}), 7.34(\mathrm{td}, J$ $=7.8,1.2 \mathrm{~Hz}, 1 \mathrm{H}), 7.32(\mathrm{~d}, J=8.4 \mathrm{~Hz}, 1 \mathrm{H}), 3.45(\mathrm{~s}, 3 \mathrm{H}) ;{ }^{13} \mathrm{C} \mathrm{NMR}\left(100 \mathrm{MHz}, \mathrm{CDCl}_{3}\right)$ $\delta 139.6,134.3,132.43,132.39,130.4,128.2,125.5,125.4,124.7,124.0,122.5,119.4$, 32.8; HRMS (ESI-TOF) $\mathrm{m} / \mathrm{z}:[\mathrm{M}+\mathrm{Na}]^{+}$Calcd for $\mathrm{C}_{13} \mathrm{H}_{11} \mathrm{NO}_{2} \mathrm{SNa}$ 268.0403; Found 268.0409 .

\section{8-(tert-butyl)-6-isopropyl-6H-dibenzo[c,e $][1,2]$ thiazine 5,5-dioxide (2d)}

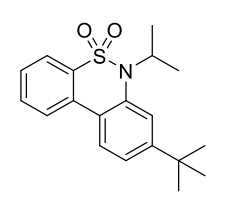

White solid; $60.5 \mathrm{mg}, 92 \%$ yield; m.p. $113.9-114.8^{\circ} \mathrm{C}$, eluent: $\mathrm{EA} / \mathrm{PE}=1: 12$ to $1: 10$; ${ }^{1} \mathrm{H}$ NMR $\left(600 \mathrm{MHz}, \mathrm{CDCl}_{3}\right) \delta 7.94(\mathrm{dd}, J=7.8,1.2 \mathrm{~Hz}, 1 \mathrm{H}), 7.88-7.86(\mathrm{~m}, 2 \mathrm{H}), 7.66$ $(\mathrm{td}, J=7.8,1.2 \mathrm{~Hz}, 1 \mathrm{H}), 7.51(\mathrm{td}, J=7.8,1.2 \mathrm{~Hz}, 1 \mathrm{H}), 7.46(\mathrm{dd}, J=8.4,1.8 \mathrm{~Hz}, 1 \mathrm{H})$, $7.42-7.42(\mathrm{~m}, 1 \mathrm{H}), 4.44$ (hept, $J=6.8 \mathrm{~Hz}, 1 \mathrm{H}), 1.38(\mathrm{~s}, 9 \mathrm{H}), 1.07(\mathrm{~d}, J=6.8 \mathrm{~Hz}, 6 \mathrm{H})$; ${ }^{13} \mathrm{C} \mathrm{NMR}\left(150 \mathrm{MHz}, \mathrm{CDCl}_{3}\right) \delta 153.3,136.7,136.2,133.2,132.3,128.0,125.8,125.3$, 125.2, 124.5, 124.3, 123.0, 54.8, 34.8, 31.1, 21.6; HRMS (ESI-TOF) m/z: [M+Na] ${ }^{+}$ Calcd for $\mathrm{C}_{19} \mathrm{H}_{23} \mathrm{NO}_{2} \mathrm{SNa} 352.1342$; Found 352.1344.

6-isopropyl-8-methyl-6H-dibenzo[c,e][1,2] thiazine 5,5-dioxide (2e)<smiles>Cc1ccc2c(c1)N(C(C)C)S(=O)(=O)c1ccccc1-2</smiles> 
White solid; $46.5 \mathrm{mg}$, $81 \%$ yield; m.p. $155.6-156.1{ }^{\circ} \mathrm{C}$, eluent: $\mathrm{EA} / \mathrm{PE}=1: 12$ to 1:10; ${ }^{1} \mathrm{H} \mathrm{NMR}\left(600 \mathrm{MHz}, \mathrm{CDCl}_{3}\right) \delta 7.93(\mathrm{dd}, J=7.8,1.2 \mathrm{~Hz}, 1 \mathrm{H}), 7.87(\mathrm{~d}, J=7.8 \mathrm{~Hz}, 1 \mathrm{H})$, $7.82(\mathrm{~d}, J=7.8 \mathrm{~Hz}, 1 \mathrm{H}), 7.65(\mathrm{td}, J=7.8,1.2 \mathrm{~Hz}, 1 \mathrm{H}), 7.51(\mathrm{td}, J=7.2,1.2 \mathrm{~Hz}, 1 \mathrm{H})$, $7.25-7.23(\mathrm{~m}, 2 \mathrm{H}), 4.41$ (hept, $J=6.8 \mathrm{~Hz}, 1 \mathrm{H}), 2.44$ (s, 3H), 1.09 (d, $J=6.8 \mathrm{~Hz}, 6 \mathrm{H})$; ${ }^{13} \mathrm{C} \mathrm{NMR}\left(100 \mathrm{MHz}, \mathrm{CDCl}_{3}\right) \delta 140.1,137.2,136.3,133.4,132.3,128.1,128.0,127.7$, 126.0, 125.5, 125.3, 123.0, 54.9, 21.6, 21.4. HRMS (ESI-TOF) m/z: $[\mathrm{M}+\mathrm{Na}]^{+} \mathrm{Calcd}$ for $\mathrm{C}_{16} \mathrm{H}_{17} \mathrm{NO}_{2} \mathrm{SNa} 310.0872$; Found: 310.0879 .

\section{6-isopropyl-8-propyl-6H-dibenzo[c,e][1,2] thiazine 5,5-dioxide (2f)}

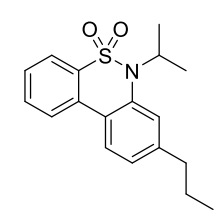

White solid; $52 \mathrm{mg}, 82 \%$ yield; m.p. $100.4-101.4{ }^{\circ} \mathrm{C}$, eluent: $\mathrm{EA} / \mathrm{PE}=1: 12$ to $1: 10 ;{ }^{1} \mathrm{H}$ NMR (600 MHz, $\left.\mathrm{CDCl}_{3}\right) \delta 7.94(\mathrm{dd}, J=7.8,1.2 \mathrm{~Hz}, 1 \mathrm{H}), 7.87(\mathrm{~d}, J=7.8 \mathrm{~Hz}, 1 \mathrm{H}), 7.85$ $(\mathrm{d}, J=8.4 \mathrm{~Hz}, 1 \mathrm{H}), 7.65(\mathrm{td}, J=7.8,1.2 \mathrm{~Hz}, 1 \mathrm{H}), 7.51(\mathrm{td}, J=7.8,1.2 \mathrm{~Hz}, 1 \mathrm{H}), 7.26-$ $7.23(\mathrm{~m}, 2 \mathrm{H}), 4.42$ (hept, $J=6.8 \mathrm{~Hz}, 1 \mathrm{H}), 2.67((\mathrm{t}, J=7.2 \mathrm{~Hz}, 2 \mathrm{H}), 1.74-1.68(\mathrm{~m}$, 2H), $1.09(\mathrm{~d}, J=6.8 \mathrm{~Hz}, 6 \mathrm{H}), 0.99(\mathrm{t}, J=7.2 \mathrm{~Hz}, 3 \mathrm{H}) ;{ }^{13} \mathrm{C} \mathrm{NMR}\left(100 \mathrm{MHz}, \mathrm{CDCl}_{3}\right) \delta$ $144.9,137.1,136.3,133.4,132.3,128.0,127.5,127.1,126.2$, 125.5, 125.3, 123.0, 54.9, 37.8, 24.2, 21.6, 13.8; HRMS (ESI-TOF) $\mathrm{m} / \mathrm{z}$ : $[\mathrm{M}+\mathrm{Na}]^{+}$Calcd for $\mathrm{C}_{18} \mathrm{H}_{21} \mathrm{NO}_{2} \mathrm{SNa}$ 338.1185; Found 338.1193.

\section{6-isopropyl-8-methoxy-6H-dibenzo $[c, e][1,2]$ thiazine 5,5-dioxide (2g)}

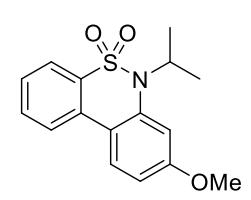

White solid; $41 \mathrm{mg}, 68 \%$ yield; m.p. $111.1-111.9{ }^{\circ} \mathrm{C}$ eluent: $\mathrm{EA} / \mathrm{PE}=1: 12$ to $1: 10 ;{ }^{1} \mathrm{H}$ NMR (400 MHz, $\left.\mathrm{CDCl}_{3}\right) \delta 7.84(\mathrm{~d}, J=7.6 \mathrm{~Hz}, 1 \mathrm{H}), 7.78(\mathrm{~d}, J=8.6 \mathrm{~Hz}, 1 \mathrm{H}), 7.74(\mathrm{~d}$, $J=8.0 \mathrm{~Hz}, 1 \mathrm{H}), 7.56(\mathrm{t}, J=7.6 \mathrm{~Hz}, 1 \mathrm{H}), 7.40(\mathrm{t}, J=7.6 \mathrm{~Hz}, 1 \mathrm{H}), 6.92-6.88(\mathrm{~m}, 2 \mathrm{H})$, 4.36 (hept, $J=6.8 \mathrm{~Hz}, 1 \mathrm{H}), 3.81(\mathrm{~s}, 3 \mathrm{H}), 1.04(\mathrm{~d}, J=6.8 \mathrm{~Hz}, 6 \mathrm{H}) ;{ }^{13} \mathrm{C} \mathrm{NMR}(100 \mathrm{MHz}$, $\left.\mathrm{CDCl}_{3}\right) \delta 160.5,138.6,135.6,133.3,132.3,127.5,126.8,125.0,123.0,121.7,113.5$, 
112.2, 55.6, 54.8, 21.6; HRMS (ESI-TOF) m/z: $[\mathrm{M}+\mathrm{Na}]^{+}$Calcd for $\mathrm{C}_{16} \mathrm{H}_{17} \mathrm{NO}_{3} \mathrm{SNa}$ 326.0821; Found 326.0825.

\section{6-isopropyl-8-phenyl-6H-dibenzo[c,e][1,2] thiazine 5,5-dioxide (2h)}

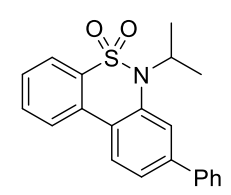

White solid; $50 \mathrm{mg}, 71 \%$ yield; m.p. $193.3-194.1{ }^{\circ} \mathrm{C}$, eluent: $\mathrm{EA} / \mathrm{PE}=1: 12$ to $1: 10 ;{ }^{1} \mathrm{H}$ NMR (600 MHz, $\left.\mathrm{CDCl}_{3}\right) \delta 8.01(\mathrm{~d}, J=9.0 \mathrm{~Hz}, 1 \mathrm{H}), 7.98(\mathrm{~d}, J=7.8 \mathrm{~Hz}, 1 \mathrm{H}), 7.94(\mathrm{~d}$, $J=7.8 \mathrm{~Hz}, 1 \mathrm{H}), 7.71-7.69(\mathrm{~m}, 1 \mathrm{H}), 7.67-7.64(\mathrm{~m}, 4 \mathrm{H}), 7.56(\mathrm{t}, J=7.2 \mathrm{~Hz}, 1 \mathrm{H}), 7.50$ $((\mathrm{t}, J=7.8 \mathrm{~Hz}, 2 \mathrm{H}), 7.43-7.04(\mathrm{~m}, 1 \mathrm{H}), 4.49$ (hept, $J=6.8 \mathrm{~Hz}, 1 \mathrm{H}), 1.14$ (d, $J=6.8$ $\mathrm{Hz}, 6 \mathrm{H}) ;{ }^{13} \mathrm{C} \mathrm{NMR}\left(150 \mathrm{MHz}, \mathrm{CDCl}_{3}\right) \delta 142.7,139.5,137.6,136.5,133.0,132.4,129.0$, 128.4, 128.1, 127.4, 127.1, 126.1, 125.9, 125.7, 125.5, 123.1, 55.1, 21.7; HRMS (ESITOF) m/z: [M+Na] ${ }^{+}$Calcd for $\mathrm{C}_{21} \mathrm{H}_{19} \mathrm{NO}_{2} \mathrm{SNa} 372.1029$; Found 372.1044.

\section{8-chloro-6-isopropyl-6H-dibenzo[c,e][1,2] thiazine 5,5-dioxide (2i)}

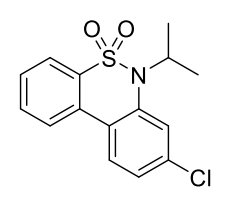

White solid; $44 \mathrm{mg}, 72 \%$ yield; m.p. $156.9-157.2{ }^{\circ} \mathrm{C}$, eluent: $\mathrm{EA} / \mathrm{PE}=1: 12$ to $1: 10 ;{ }^{1} \mathrm{H}$ NMR (400 MHz, $\left.\mathrm{CDCl}_{3}\right) \delta 7.95(\mathrm{~d}, J=7.6 \mathrm{~Hz}, 1 \mathrm{H}), 7.88-7.84(\mathrm{~m}, 2 \mathrm{H}), 7.68(\mathrm{t}, J=$ $7.6 \mathrm{~Hz}, 1 \mathrm{H}), 7.56$ (t, $J=7.6 \mathrm{~Hz}, 1 \mathrm{H}), 7.45(\mathrm{~s}, 1 \mathrm{H}), 7.40-7.38$ (m, 1H), 4.43 (hept, $J=$ $6.8 \mathrm{~Hz}, 1 \mathrm{H}), 1.13(\mathrm{~d}, J=6.8 \mathrm{~Hz}, 6 \mathrm{H}) ;{ }^{13} \mathrm{C} \mathrm{NMR}\left(100 \mathrm{MHz}, \mathrm{CDCl}_{3}\right) \delta$ 138.4, 136.4, 135.1, 132.5, 132.3, 128.8, 127.3, 127.1, 126.9, 126.8, 125.5, 123.0, 55.3, 21.6; HRMS (ESI-TOF) m/z: $[\mathrm{M}+\mathrm{Na}]^{+}$Calcd for $\mathrm{C}_{15} \mathrm{H}_{14} \mathrm{ClNO}_{2} \mathrm{SNa} 330.0326$; Found 330.0322.

\section{8-fluoro-6-isopropyl-6H-dibenzo[c,e $][1,2]$ thiazine 5,5-dioxide (2j)}<smiles>CC(C)N(c1cc(F)ccc1-c1ccccc1S(=O)(=O)O)C(C)C</smiles>

White solid; $37 \mathrm{mg}, 64 \%$ yield; m.p. $83.5-84{ }^{\circ} \mathrm{C}$, eluent: $\mathrm{EA} / \mathrm{PE}=1: 12$ to $1: 10 ;{ }^{1} \mathrm{H}$ NMR $\left(400 \mathrm{MHz}, \mathrm{CDCl}_{3}\right) \delta 7.96-7.91(\mathrm{~m}, 2 \mathrm{H}), 7.84(\mathrm{~d}, J=8.0 \mathrm{~Hz}, 1 \mathrm{H}), 7.68(\mathrm{t}, J=7.6 \mathrm{~Hz}$, 1H), 7.55 (t, $J=7.6 \mathrm{~Hz}, 1 \mathrm{H}), 7.19-7.13(\mathrm{~m}, 2 \mathrm{H}), 4.46$ (hept, $J=6.8 \mathrm{~Hz}, 1 \mathrm{H}), 1.14$ (d, 
$J=6.8 \mathrm{~Hz}, 6 \mathrm{H}) ;{ }^{13} \mathrm{C} \mathrm{NMR}\left(100 \mathrm{MHz}, \mathrm{CDCl}_{3}\right) \delta 162.6(\mathrm{~d}, J=249.6 \mathrm{~Hz}), 139.0,138.8$, 136.0, 132.5, 128.4, $127.4(\mathrm{~d}, J=9.6 \mathrm{~Hz}), 125.5,125.0(\mathrm{~d}, J=3.2 \mathrm{~Hz}), 123.0,114.5$ (d, $J=21.7 \mathrm{~Hz}), 113.9(\mathrm{~d}, J=23.3 \mathrm{~Hz}), 55.0,21.6 ;{ }^{19} \mathrm{~F} \mathrm{NMR}\left(375 \mathrm{MHz}, \mathrm{CDCl}_{3}\right) \delta$-109.4; HRMS (ESI-TOF) m/z: $[\mathrm{M}+\mathrm{Na}]^{+}$Calcd for $\mathrm{C}_{15} \mathrm{H}_{14} \mathrm{FNO}_{2} \mathrm{SNa} 314.0621$; Found 314.0623.

\section{6-isopropyl-8-(trifluoromethyl)-6H-dibenzo[c,e][1,2]thiazine 5,5-dioxide (2k)}

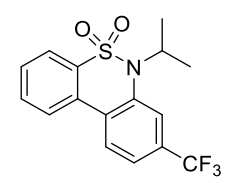

White solid; $45 \mathrm{mg}, 66 \%$ yield; m.p. $183.4-185.5{ }^{\circ} \mathrm{C}$, eluent: $\mathrm{EA} / \mathrm{PE}=1: 12$ to $1: 10 ;{ }^{1} \mathrm{H}$ NMR (400 MHz, $\left.\mathrm{CDCl}_{3}\right) \delta 8.08(\mathrm{~d}, J=8.0 \mathrm{~Hz}, 1 \mathrm{H}), 8.00(\mathrm{dd}, J=7.6,1.2 \mathrm{~Hz}, 1 \mathrm{H}), 7.94$ $(\mathrm{d}, J=8.0 \mathrm{~Hz}, 1 \mathrm{H}), 7.74(\mathrm{td}, J=7.6,1.2 \mathrm{~Hz}, 1 \mathrm{H}), 7.69-7.62(\mathrm{~m}, 3 \mathrm{H}), 4.47$ (hept, $J=$ $6.8 \mathrm{~Hz}, 1 \mathrm{H}), 1.14(\mathrm{~d}, J=6.8 \mathrm{~Hz}, 6 \mathrm{H}) ;{ }^{13} \mathrm{C} \mathrm{NMR}\left(150 \mathrm{MHz}, \mathrm{CDCl}_{3}\right) \delta 137.3(\mathrm{~d}, J=$ $118.6 \mathrm{~Hz}), 132.6,131.8,131.7,131.5(\mathrm{~d}, J=33.2 \mathrm{~Hz}), 129.6,126.2(\mathrm{~d}, J=49.4 \mathrm{~Hz}), 124.2$, $124.0(\mathrm{q}, J=3.8 \mathrm{~Hz}), 123.6(\mathrm{q}, J=3.4 \mathrm{~Hz}), 123.2,122.4,55.4,21.6 ;{ }^{19} \mathrm{~F}$ NMR $(375 \mathrm{MHz}$, $\left.\mathrm{CDCl}_{3}\right) \delta$-62.8; (ESI-TOF) m/z: $[\mathrm{M}+\mathrm{Na}]^{+}$Calcd for $\mathrm{C}_{16} \mathrm{H}_{14} \mathrm{~F}_{3} \mathrm{NO}_{2} \mathrm{SNa}$ 364.0590; Found 364.0593.

\section{8-chloro-6-methyl-6H-dibenzo[c,e][1,2] thiazine 5,5-dioxide (2l) ${ }^{8}$}<smiles>Cn1c2ccccc2c2ccc(Cl)cc21</smiles>

White solid; $39 \mathrm{mg}, 70 \%$ yield; m.p. $129.6-131.0{ }^{\circ} \mathrm{C}$, eluent: $\mathrm{EA} / \mathrm{PE}=1: 12$ to $1: 10 ;{ }^{1} \mathrm{H}$ NMR (600 MHz, $\left.\mathrm{CDCl}_{3}\right) \delta 8.01(\mathrm{dd}, J=7.8,1.2 \mathrm{~Hz}, 1 \mathrm{H}), 7.94(\mathrm{~d}, J=8.4 \mathrm{~Hz}, 1 \mathrm{H}), 7.92$ $(\mathrm{d}, J=7.8 \mathrm{~Hz}, 1 \mathrm{H}), 7.72(\mathrm{td}, J=7.8,1.2 \mathrm{~Hz}, 1 \mathrm{H}), 7.59$ (td, $J=7.2,0.6 \mathrm{~Hz}, 1 \mathrm{H}), 7.32$ $-7.29(\mathrm{~m}, 2 \mathrm{H}), 3.46(\mathrm{~s}, 3 \mathrm{H}) ;{ }^{13} \mathrm{C}$ NMR $\left(100 \mathrm{MHz}, \mathrm{CDCl}_{3}\right) \delta 140.2,136.0,133.9,132.6$, 131.5, 128.5, 126.6, 125.3, 124.7, 122.4, 122.2, 119.0, 32.2; HRMS (APCI-TOF) m/z: $[\mathrm{M}+\mathrm{H}]^{+}$Calcd for $\mathrm{C}_{13} \mathrm{H}_{11} \mathrm{ClNO}_{2} \mathrm{~S}$ 280.0194; Found 280.0194.

\section{6-isopropyl-7,9-dimethyl-6H-dibenzo[c,e][1,2] thiazine 5,5-dioxide (2m)}




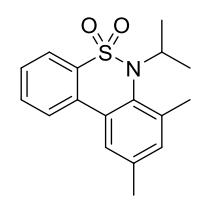

White solid; $50.6 \mathrm{mg}, 84 \%$ yield, eluent: $\mathrm{EA} / \mathrm{PE}=1: 12$ to $1: 10 ;{ }^{1} \mathrm{H}$ NMR $(600 \mathrm{MHz}$, $\left.\mathrm{CDCl}_{3}\right) \delta 7.86(\mathrm{dd}, J=7.8,1.2 \mathrm{~Hz}, 1 \mathrm{H}), 7.81(\mathrm{~d}, J=7.8 \mathrm{~Hz}, 1 \mathrm{H}), 7.58(\mathrm{td}, J=7.8,1.2$ Hz, 1H), $7.46-7.43(\mathrm{~m}, 2 \mathrm{H}), 7.08(\mathrm{~s}, 1 \mathrm{H}), 3.56$ (hept, $J=6.8 \mathrm{~Hz}, 1 \mathrm{H}), 2.40(\mathrm{~s}, 3 \mathrm{H})$, $2.33(\mathrm{~s}, 3 \mathrm{H}), 1.24(\mathrm{~d}, J=6.8 \mathrm{~Hz}, 3 \mathrm{H}), 0.93(\mathrm{~d}, J=6.8 \mathrm{~Hz}, 3 \mathrm{H}) ;{ }^{13} \mathrm{C}$ NMR $(150 \mathrm{MHz}$, $\left.\mathrm{CDCl}_{3}\right) \delta 137.0,136.8,136.7,136.3,134.0,133.2,132.3,128.5,128.3,125.5,123.9$, 123.6, 57.7, 22.0, 21.2, 20.2, 18.6; HRMS (ESI-TOF) m/z: $[\mathrm{M}+\mathrm{Na}]^{+}$Calcd for $\mathrm{C}_{17} \mathrm{H}_{19} \mathrm{NO}_{2} \mathrm{SNa}$ 24.1029; Found 324.1030.

A mixture of 6-isopropyl-7-methyl-6H-dibenzo[c,e][1,2]thiazine5,5-dioxide and 6isopropyl-9-methyl-6H-dibenzo[c,e][1,2] thiazine 5,5-dioxide $(2 \mathrm{n} / 2 \mathrm{n}$ ')

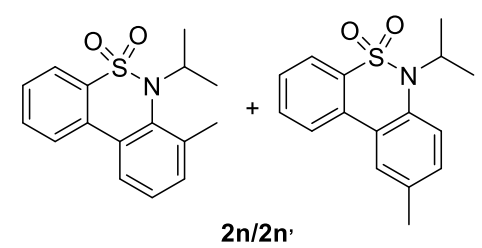

Isomer with more polarity: isomer with less polarity (1: 1.04); white solid; $44 \mathrm{mg}, 77 \%$ yield, eluent: $\mathrm{EA} / \mathrm{PE}=1: 12$ to $1: 10 ;{ }^{1} \mathrm{H}$ NMR $(400 \mathrm{MHz}$, Acetone-d $) \delta 8.16-8.13(\mathrm{~m}$, 2H), 7.97 (s, 1H), 7.95-7.91 (m, 3H), 7.83-7.80 (m, 2H), 7.70-7.66 (m, 2H), $7.46-7.40$ (m, 3H), $7.36(\mathrm{dd}, J=8.4,1.6 \mathrm{~Hz}, 1 \mathrm{H}), 4.38$ (hept, $J=6.8 \mathrm{~Hz}, 1 \mathrm{H}), 3.70$ (hept, $J=6.8$ $\mathrm{Hz}, 1 \mathrm{H}), 2.52(\mathrm{~s}, 3 \mathrm{H}), 2.48(\mathrm{~s}, 3 \mathrm{H}), 1.38(\mathrm{~d}, J=6.8 \mathrm{~Hz}, 3 \mathrm{H}), 1.05(\mathrm{~d}, J=6.8 \mathrm{~Hz}, 6 \mathrm{H})$, $0.96(\mathrm{~d}, J=6.8 \mathrm{~Hz}, 3 \mathrm{H}) ;{ }^{13} \mathrm{C} \mathrm{NMR}\left(150 \mathrm{MHz}, \mathrm{CDCl}_{3}\right) \delta 139.5,137.1,136.8,136.62$, $136.57,134.4,133.9,133.4,132.4,132.32,132.28,130.4,128.9,128.52,128.46,128.3$, $127.4,127.3,126.2,125.6,125.5,123.5,123.4,123.1,57.9,54.6,22.1,21.4,21.3,20.1$, 18.7; HRMS (ESI-TOF) m/z: [M+Na] $]^{+}$Calcd for $\mathrm{C}_{16} \mathrm{H}_{17} \mathrm{NO}_{2} \mathrm{SNa}$ 310.0872; Found 310.0871 .

9-fluoro-6-isopropyl-6H-dibenzo[c,e][1,2]thiazine5,5-dioxide and 7-fluoro-6isopropyl-6H-dibenzo $[c, e][1,2]$ thiazine 5,5 -dioxide $(20 / 20$ ') 


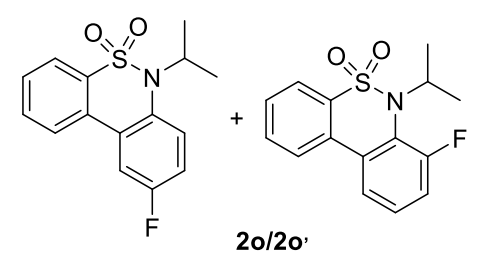

Isomer with more polarity: White solid; $14.8 \mathrm{mg}, 25.5 \%$ yield, eluent: $\mathrm{EA} / \mathrm{PE}=1: 10$; ${ }^{1} \mathrm{H} \mathrm{NMR}\left(400 \mathrm{MHz}, \mathrm{CDCl}_{3}\right) \delta 7.97(\mathrm{dd}, J=7.6,1.2 \mathrm{~Hz}, 1 \mathrm{H}), 7.84(\mathrm{~d}, J=7.6 \mathrm{~Hz}, 1 \mathrm{H})$, $7.70(\mathrm{td}, J=7.6,1.2 \mathrm{~Hz}, 1 \mathrm{H}), 7.64-7.57(\mathrm{~m}, 2 \mathrm{H}), 7.43(\mathrm{dd}, J=8.8,5.2 \mathrm{~Hz}, 1 \mathrm{H}), 7.16$ (ddd, $J=9.4,7.4,2.8 \mathrm{~Hz}, 1 \mathrm{H}), 4.41$ (hept, $J=6.8 \mathrm{~Hz}, 1 \mathrm{H}), 1.03(\mathrm{~d}, J=6.8 \mathrm{~Hz}, 6 \mathrm{H}$ ); ${ }^{13} \mathrm{C}$ NMR $\left(150 \mathrm{MHz}, \mathrm{CDCl}_{3}\right) \delta 161.3(\mathrm{~d}, J=245.6 \mathrm{~Hz}), 136.4,132.8(\mathrm{~d}, J=2.8 \mathrm{~Hz})$, 132.6, 132.3(d, $J=2.1 \mathrm{~Hz}), 130.7(\mathrm{~d}, J=8.4 \mathrm{~Hz}), 129.6(\mathrm{~d}, J=8.6 \mathrm{~Hz}), 129.2,125.7$, $123.3,116.6(\mathrm{~d}, J=22.6 \mathrm{~Hz}), 112.4(\mathrm{~d}, J=24 \mathrm{~Hz}), 54.8,21.3 ;{ }^{19} \mathrm{~F}$ NMR $(375 \mathrm{MHz}$, CDCl3) $\delta$-113.2.HRMS (ESI-TOF) $\mathrm{m} / \mathrm{z}:[\mathrm{M}+\mathrm{Na}]^{+}$Calcd for $\mathrm{C}_{15} \mathrm{H}_{14} \mathrm{FNO}_{2} \mathrm{SNa}$ 314.0621; Found 314.0618.

Isomer with less polarity: White solid; $16.6 \mathrm{mg}, 28.5 \%$ yield, eluent: $\mathrm{EA} / \mathrm{PE}=1: 12$; ${ }^{1} \mathrm{H}$ NMR $\left(400 \mathrm{MHz}, \mathrm{CDCl}_{3}\right) \delta 7.97(\mathrm{dd}, J=8.0,1.2 \mathrm{~Hz}, 1 \mathrm{H}), 7.89(\mathrm{~d}, J=7.6 \mathrm{~Hz}, 1 \mathrm{H})$, 7.72-7.68 (m, 2H), $7.59(\mathrm{td}, J=7.6,0.8 \mathrm{~Hz}, 1 \mathrm{H}), 7.39(\mathrm{td}, J=8.0,5.2 \mathrm{~Hz}, 1 \mathrm{H}), 7.23-$ $7.20(\mathrm{~m}, 1 \mathrm{H}), 4.17-4.07(\mathrm{~m}, 1 \mathrm{H}), 1.16(\mathrm{~s}, 6 \mathrm{H}) ;{ }^{13} \mathrm{C} \mathrm{NMR}\left(150 \mathrm{MHz}, \mathrm{CDCl}_{3}\right) \delta 157.8$ $(\mathrm{d}, J=249.0 \mathrm{~Hz}), 136.7,132.53(\mathrm{~d}, J=3.2 \mathrm{~Hz}), 132.51,130.8,129.1,127.9$ (d, $J=8.6$ $\mathrm{Hz}), 127.1$ (d, $J=15.0 \mathrm{~Hz}), 125.9,123.4,121.0(\mathrm{~d}, J=3.4 \mathrm{~Hz}), 117.0(\mathrm{~d}, J=20.6 \mathrm{~Hz})$, 57.1, 23.4; ${ }^{19} \mathrm{~F}$ NMR $\left(375 \mathrm{MHz}, \mathrm{CDCl}_{3}\right) \delta-114.1$; HRMS (ESI-TOF) m/z: $[\mathrm{M}+\mathrm{Na}]^{+}$ Calcd for $\mathrm{C}_{15} \mathrm{H}_{14} \mathrm{FNO}_{2} \mathrm{SNa} 314.0621$; Found 314.0620.

\section{0-fluoro-6-isopropyl-6H-dibenzo[c,e][1,2] thiazine 5,5-dioxide (2p)}

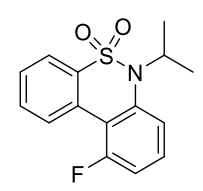

White solid; $26 \mathrm{mg}, 45 \%$ yield; m.p. $128.6-130{ }^{\circ} \mathrm{C}$, eluent: $\mathrm{EA} / \mathrm{PE}=1: 12$ to $1: 10 ;{ }^{1} \mathrm{H}$ NMR $\left(400 \mathrm{MHz}, \mathrm{CDCl}_{3}\right) \delta 8.18(\mathrm{~d}, J=8.0 \mathrm{~Hz}, 1 \mathrm{H}), 7.99(\mathrm{~d}, J=7.6 \mathrm{~Hz}, 1 \mathrm{H}), 7.68(\mathrm{t}, J$ $=8.0 \mathrm{~Hz}, 1 \mathrm{H}), 7.57(\mathrm{t}, J=7.6 \mathrm{~Hz}, 1 \mathrm{H}), 7.44-7.38(\mathrm{~m}, 1 \mathrm{H}), 7.28(\mathrm{~d}, J=8.4 \mathrm{~Hz}, 1 \mathrm{H})$, $7.19(\mathrm{dd}, J=10.8,8.4 \mathrm{~Hz}, 1 \mathrm{H}), 4.40$ (hept, $J=6.8 \mathrm{~Hz}, 1 \mathrm{H}), 1.07$ (d, $J=6.8 \mathrm{~Hz}, 6 \mathrm{H})$; ${ }^{13} \mathrm{C} \mathrm{NMR}\left(150 \mathrm{MHz}, \mathrm{CDCl}_{3}\right) \delta 160.3(\mathrm{~d}, J=252.2 \mathrm{~Hz}, \mathrm{H}), 138.7(\mathrm{~d}, J=4.2 \mathrm{~Hz}), 136.7$, 
132.1, 129.7(d, $J=10.8 \mathrm{~Hz}), 129.17$ (d, $J=15.8 \mathrm{~Hz}), 129.16(\mathrm{~d}, J=3.3 \mathrm{~Hz}), 128.8$, $123.2(\mathrm{~d}, J=3.2 \mathrm{~Hz}), 123.0,117.9$ (d, $J=11.2 \mathrm{~Hz}), 115.0(\mathrm{~d}, J=23.6 \mathrm{~Hz}), 55.0,21.5$;

${ }^{19} \mathrm{~F}$ NMR $\left(375 \mathrm{MHz}, \mathrm{CDCl}_{3}\right) \delta-112.1$; HRMS (ESI-TOF) $\mathrm{m} / \mathrm{z}:[\mathrm{M}+\mathrm{Na}]^{+}$Calcd for $\mathrm{C}_{15} \mathrm{H}_{14} \mathrm{FNO}_{2} \mathrm{SNa}$ 314.0621; Found 314.0618.

6-isopropyl-6,7-dihydrodibenzo[d,f][1,2] thiazepine 5,5-dioxide (2q) ${ }^{10}$

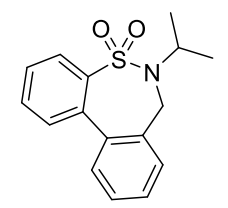

White solid; $23 \mathrm{mg}, 40 \%$ yield, m.p. 139.6-140.2 ${ }^{\circ} \mathrm{C}$, eluent: $\mathrm{EA} / \mathrm{PE}=1: 12 ;{ }^{1} \mathrm{H}$ NMR $\left(400 \mathrm{MHz}, \mathrm{CDCl}_{3}\right) \delta 8.03(\mathrm{~d}, J=7.8 \mathrm{~Hz}, 1 \mathrm{H}), 7.68(\mathrm{t}, J=7.6 \mathrm{~Hz}, 1 \mathrm{H}), 7.60(\mathrm{~d}, J=7.6$ Hz, 1H), 7.54 (t, $J=7.6 \mathrm{~Hz}, 1 \mathrm{H}), 7.47$ (s, 2H), 7.42 (s, 2H), 4.47 (hept, $J=6.8 \mathrm{~Hz}, 1 \mathrm{H}$ ), $3.84(\mathrm{~s}, 2 \mathrm{H}), 1.23(\mathrm{~d}, J=6.8 \mathrm{~Hz}, 6 \mathrm{H}) ;{ }^{13} \mathrm{C} \mathrm{NMR}\left(150 \mathrm{MHz}, \mathrm{CDCl}_{3}\right) \delta 140.8,139.2$, 138.0, 134.1, 132.8, 130.1, 129.6, 129.2, 129.0, 128.4, 128.3, 126.2, 51.0, 45.4, 19.8; HRMS (ESI-TOF) m/z: $[\mathrm{M}+\mathrm{Na}]^{+}$Calcd for $\mathrm{C}_{16} \mathrm{H}_{17} \mathrm{NO}_{2} \mathrm{SNa} 310.0872$; Found 310.0873 .

6-isopropyl-7-methyl-6,7-dihydrodibenzo $[d, f][1,2]$ thiazepine 5,5-dioxide (2r)

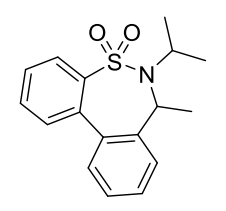

White solid; $24.7 \mathrm{mg}, 41 \%$ yield; m.p. $147.3-148.2{ }^{\circ} \mathrm{C}$, eluent: $\mathrm{EA} / \mathrm{PE}=1: 12 ;{ }^{1} \mathrm{H} \mathrm{NMR}$ $\left(400 \mathrm{MHz}, \mathrm{CDCl}_{3}\right) \delta 8.11(\mathrm{dd}, J=8.0,1.2 \mathrm{~Hz}, 1 \mathrm{H}), 7.68(\mathrm{td}, J=7.6,1.2 \mathrm{~Hz}, 1 \mathrm{H}), 7.60$ $(\mathrm{dd}, J=7.6,1.2 \mathrm{~Hz}, 1 \mathrm{H}), 7.55-7.51(\mathrm{~m}, 2 \mathrm{H}), 7.46(\mathrm{td}, J=7.2,1.2 \mathrm{~Hz}, 1 \mathrm{H}), 7.40(\mathrm{td}, J$ $=7.2,1.6 \mathrm{~Hz}, 1 \mathrm{H}), 7.31(\mathrm{dd}, J=7.2,1.2 \mathrm{~Hz}, 1 \mathrm{H}), 4.60$ (hept, $J=6.8 \mathrm{~Hz}, 1 \mathrm{H}), 4.48$ (q, $J=6.8 \mathrm{~Hz}, 1 \mathrm{H}), 1.40(\mathrm{~d}, J=6.8 \mathrm{~Hz}, 3 \mathrm{H}), 1.33(\mathrm{~d}, J=6.8 \mathrm{~Hz}, 3 \mathrm{H}), 0.96(\mathrm{~d}, J=6.8 \mathrm{~Hz}$, $3 \mathrm{H}) ;{ }^{13} \mathrm{C} \mathrm{NMR}\left(150 \mathrm{MHz}, \mathrm{CDCl}_{3}\right) \delta 140.9,140.1,139.1,138.5,133.0,129.7,129.6$, 129.2, 128.8, 128.0, 126.7, 55.4, 49.2, 22.4, 21.9, 21.5; HRMS (ESI-TOF) m/z: $[\mathrm{M}+\mathrm{Na}]^{+}$Calcd for $\mathrm{C}_{17} \mathrm{H}_{19} \mathrm{NO}_{2} \mathrm{SNa}$ 324.1029; Found 324.1026.

6-(tert-butyl)-6,7-dihydrodibenzo $[d, f][1,2]$ thiazepine 5,5-dioxide (2s) 


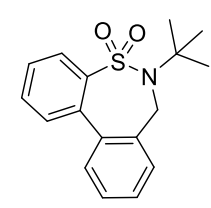

White solid; $30.7 \mathrm{mg}, 51 \%$ yield, eluent: $\mathrm{EA} / \mathrm{PE}=1: 12 ;{ }^{1} \mathrm{H} \mathrm{NMR}\left(600 \mathrm{MHz}, \mathrm{CDCl}_{3}\right) \delta$ $8.09(\mathrm{dd}, J=7.8,1.2 \mathrm{~Hz}, 1 \mathrm{H}), 7.67(\mathrm{td}, J=7.8,1.2 \mathrm{~Hz}, 1 \mathrm{H}), 7.58(\mathrm{dd}, J=7.8,1.2 \mathrm{~Hz}, 1 \mathrm{H})$, $7.54(\mathrm{td}, J=7.8,1.2 \mathrm{~Hz}, 1 \mathrm{H}), 7.49-7.46(\mathrm{~m}, 2 \mathrm{H}), 7.42-7.41(\mathrm{~m}, 2 \mathrm{H}), 3.95(\mathrm{~s}, 2 \mathrm{H})$,

$1.53(\mathrm{~s}, 9 \mathrm{H}) ;{ }^{13} \mathrm{C}$ NMR $\left(150 \mathrm{MHz}, \mathrm{CDCl}_{3}\right) \delta 140.5,139.2,138.3,135.0,132.8,130.4$, 129.1, 129.01, 128.98, 128.3 127.8, 127.5, 61.6, 48.8, 29.6; HRMS (ESI-TOF) m/z: $[\mathrm{M}+\mathrm{Na}]^{+}$Calcd for $\mathrm{C}_{17} \mathrm{H}_{19} \mathrm{NO}_{2} \mathrm{SNa} 324.1029$; Found 324.1028.

\section{6-(tert-butyl)-7-methyl-6,7-dihydrodibenzo[d,f][1,2] thiazepine 5,5-dioxide (2t)}

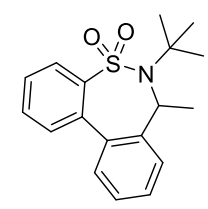

White solid; $28 \mathrm{mg}, 44 \%$ yield, eluent: $\mathrm{EA} / \mathrm{PE}=1: 12 ;{ }^{1} \mathrm{H} \mathrm{NMR}\left(600 \mathrm{MHz}, \mathrm{CDCl}_{3}\right) \delta$ $8.17(\mathrm{dd}, J=7.8,1.2 \mathrm{~Hz}, 1 \mathrm{H}), 7.67(\mathrm{td}, J=7.2 \mathrm{~Hz}, 1 \mathrm{H}), 7.57-7.52(\mathrm{~m}, 3 \mathrm{H}), 7.47(\mathrm{t}, J=$ $7.2 \mathrm{~Hz}, 1 \mathrm{H}), 7.40(\mathrm{t}, J=7.2 \mathrm{~Hz}, 1 \mathrm{H}), 7.34(\mathrm{~d}, J=7.2 \mathrm{~Hz}, 1 \mathrm{H}), 4.76(\mathrm{q}, J=6.6 \mathrm{~Hz}, 1 \mathrm{H})$, $1.64(\mathrm{~s}, 9 \mathrm{H}), 0.90(\mathrm{~d}, J=6.6 \mathrm{~Hz}, 3 \mathrm{H}) ;{ }^{13} \mathrm{C} \mathrm{NMR}\left(150 \mathrm{MHz}, \mathrm{CDCl}_{3}\right) \delta 141.1,140.5$, 140.0, 138.7, 133.0, 130.0, 129.2, 129.0, 128.85, 128.84, 128.0, 127.8, 63.3, 57.3, 31.0, 21.3; HRMS (ESI-TOF) m/z: [M+Na] ${ }^{+}$Calcd for $\mathrm{C}_{18} \mathrm{H}_{21} \mathrm{NO}_{2} \mathrm{SNa}$ 338.1185; Found 338.1184 .

\section{6-methyl-6,7-dihydrodibenzo[ $[d, f][1,2]$ thiazepine5,5-dioxide (2u)}

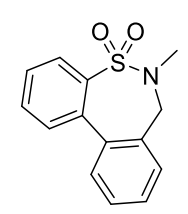

$3.1 \mathrm{mg}, 6 \%$ yield, eluent: $\mathrm{EA} / \mathrm{PE}=1: 12 ;{ }^{1} \mathrm{H} \mathrm{NMR}\left(400 \mathrm{MHz}, \mathrm{CDCl}_{3}\right) \delta 8.00(\mathrm{dd}, J=$ 8.0, 1.2 Hz, 1H), $7.72(\mathrm{td}, J=7.6,1.2 \mathrm{~Hz}, 1 \mathrm{H}), 7.61(\mathrm{dd}, J=7.6,1.2 \mathrm{~Hz}, 1 \mathrm{H}), 7.56(\mathrm{td}$, $J=7.6,1.2 \mathrm{~Hz}, 1 \mathrm{H}), 7.52-7.43(\mathrm{~m}, 4 \mathrm{H}), 3.77(\mathrm{~s}, 2 \mathrm{H}), 2.88(\mathrm{~s}, 3 \mathrm{H}) ;{ }^{13} \mathrm{C}$ NMR $(100$ $\left.\mathrm{MHz}, \mathrm{CDCl}_{3}\right) \delta 140.8,139.4,134.8,133.4,133.3,130.2,129.9,129.5,129.0,128.5$, 128.3, 127.5, 56.1, 39.0; HRMS (ESI-TOF) m/z: $[\mathrm{M}+\mathrm{Na}]^{+}$Calcd for $\mathrm{C}_{14} \mathrm{H}_{13} \mathrm{NO}_{2} \mathrm{SNa}$ 282.0559; Found 282.0560. 


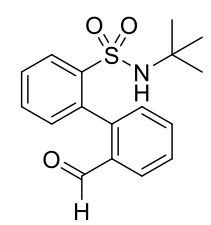

White solid; $5 \mathrm{mg}, 8 \%$ yield, eluent: EA/PE $=1: 10 ;{ }^{1} \mathrm{H}$ NMR $\left(600 \mathrm{MHz}, \mathrm{CDCl}_{3}\right) \delta 9.68$ (s, 1H), 8.19 (dd, $J=7.8,1.2 \mathrm{~Hz}, 1 \mathrm{H}), 8.06(\mathrm{dd}, J=7.8,1.2 \mathrm{~Hz}, 1 \mathrm{H}), 7.64(\mathrm{td}, J$ $=7.2,1.2 \mathrm{~Hz} \mathrm{1H}), 7.60(\mathrm{dd}, J=7.2,1.2 \mathrm{~Hz}, 1 \mathrm{H}), 7.59-7.56(\mathrm{~m}, 2 \mathrm{H}), 7.42(\mathrm{~d}, J=7.2 \mathrm{~Hz}$, 1H), $7.33(\mathrm{dd}, J=7.2,1.2 \mathrm{~Hz}, 1 \mathrm{H}), 3.54(\mathrm{~s}, 1 \mathrm{H}), 1.12(\mathrm{~s}, 9 \mathrm{H}) ;{ }^{13} \mathrm{C}$ NMR $(150 \mathrm{MHz}$, $\left.\mathrm{CDCl}_{3}\right) \delta 191.0,142.2,141.9,136.4,134.5,132.7,132.4,131.8,131.2,128.9,128.7$, 128.4, 127.4, 55.1, 30.0; HRMS (ESI-TOF) m/z: $[\mathrm{M}+\mathrm{Na}]^{+}$Calcd for $\mathrm{C}_{17} \mathrm{H}_{19} \mathrm{NO}_{3} \mathrm{SNa}$ 340.0978; Found 340.0971. 


\section{4) Synthesis of 6-methyl-6,7-dihydrodibenzo $[d, f][1,2]$ thiazepine 5 ,}

\section{5-dioxide $(2 \mathrm{u})^{11-12}$}

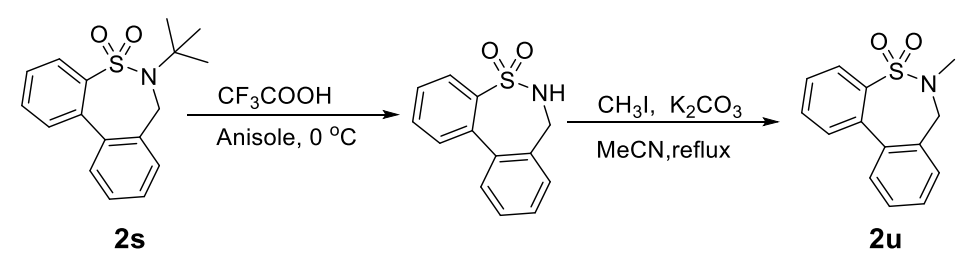

Scheme S1. Synthesis of $2 u$ from $2 \mathbf{s}$

A solution of $2 \mathrm{~s}(24 \mathrm{mg}, 0.08 \mathrm{mmol})$ in anisole $(0.2 \mathrm{~mL})$ and $\mathrm{CF}_{3} \mathrm{COOH}(0.5 \mathrm{~mL})$ was stirred for $0.5 \mathrm{~h}$ at $0{ }^{\circ} \mathrm{C}$ After dilution with EA, the solution was neutralized at $0{ }^{\circ} \mathrm{C}$ with saturated aqueous $\mathrm{Na}_{2} \mathrm{CO}_{3}$. The organic phase was dried $\left(\mathrm{Na}_{2} \mathrm{SO}_{4}\right)$, anisole was removed in vacuo and the crude product was used immediately for the next step without further purification. To a solution of the crude product and $\mathrm{K}_{2} \mathrm{CO}_{3}(27.6 \mathrm{mg}, 0.2 \mathrm{mmol})$ in dry $\mathrm{CH}_{3} \mathrm{CN}(1 \mathrm{~mL})$ was added $\mathrm{CH}_{3} \mathrm{I}(10 \mu \mathrm{L}, 0.16 \mathrm{mmol})$, and the mixture was refluxed in oil bath overnight. The reaction mixture was cooled to rt, and the solvent was removed in vacuo. The residue was partitioned between EA and water, and the aqueous layer was extracted with EA $(3 \times 2 \mathrm{~mL})$. The combined organic phase was washed with brine, dried over $\mathrm{Na}_{2} \mathrm{SO}_{4}$, filtered and concentrated, purified by flash silica gel chromatography (EtOAc/petroleum ether 1:7) to give $\mathbf{2 u}(16.1 \mathrm{mg}$, $78 \%$ in two steps).

\section{6-methyl-6,7-dihydrodibenzo[ $[d, f][1,2]$ thiazepine5,5-dioxide $(2 \mathrm{u})^{13}$}

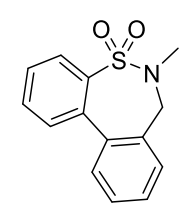

${ }^{1} \mathrm{H}$ NMR $\left(400 \mathrm{MHz}, \mathrm{CDCl}_{3}\right) \delta 8.00(\mathrm{dd}, J=8.0,1.2 \mathrm{~Hz}, 1 \mathrm{H}), 7.72(\mathrm{td}, J=7.6,1.2 \mathrm{~Hz}$, 1H), $7.61(\mathrm{dd}, J=7.6,1.2 \mathrm{~Hz}, 1 \mathrm{H}), 7.56(\mathrm{td}, J=7.6,1.2 \mathrm{~Hz}, 1 \mathrm{H}), 7.52-7.43(\mathrm{~m}, 4 \mathrm{H})$, $3.77(\mathrm{~s}, 2 \mathrm{H}), 2.88(\mathrm{~s}, 3 \mathrm{H}) ;{ }^{13} \mathrm{C} \mathrm{NMR}\left(100 \mathrm{MHz}, \mathrm{CDCl}_{3}\right) \delta 140.8,139.4,134.8,133.4$, 133.3, 130.2, 129.9, 129.5, 129.0, 128.5, 128.3, 127.5, 56.1, 39.0; HRMS (ESI-TOF) $\mathrm{m} / \mathrm{z}:[\mathrm{M}+\mathrm{Na}]^{+}$Calcd for $\mathrm{C}_{14} \mathrm{H}_{13} \mathrm{NO}_{2} \mathrm{SNa} 282.0559$; Found 282.0560. 


\section{5) Synthesis of 2-benzyl-benzenesulfonamide derivatives ${ }^{11}$}

\section{General procedure:}

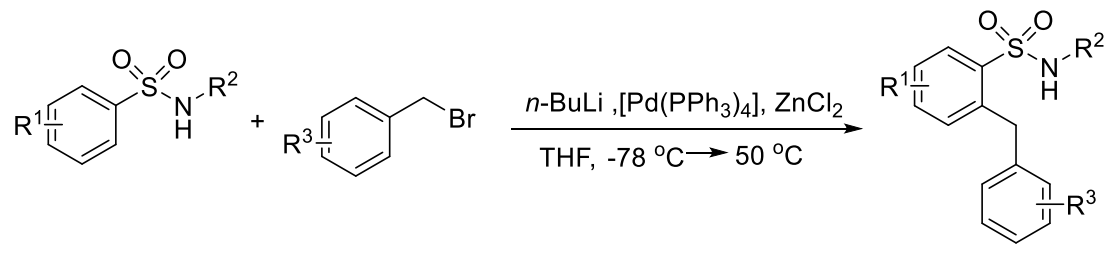

To the solution of corresponding starting material (3.0 mmol, 1 equiv) in dry THF( 8.0 $\mathrm{mL})$ was added $n$-BuLi $\left(6.3 \mathrm{mmol}, 2.1\right.$ equiv) at $-78^{\circ} \mathrm{C}$. The mixture was stirred for 30 min at $0{ }^{\circ} \mathrm{C}$ and then cooled to $-78{ }^{\circ} \mathrm{C} . \mathrm{ZnCl}_{2}(6.3 \mathrm{mmol}, 2.5$ equiv) in dry THF ( 6.0 $\mathrm{mL}$ ) was added. The mixture was stirred for another $15 \mathrm{~min}$ and allowed to reach $0{ }^{\circ} \mathrm{C}$. Then benzyl bromide (3.6 mmol, 1.2 equiv) and $\left[\mathrm{Pd}\left(\mathrm{PPh}_{3}\right)_{4}\right](0.17 \mathrm{~g}, 5 \mathrm{~mol} \%)$ were added. The mixture was heated in oil bath to $50^{\circ} \mathrm{C}$, stirred overnight, then poured into $2 \mathrm{M} \mathrm{HCl}(12 \mathrm{~mL})$, and extracted with $\mathrm{CH}_{2} \mathrm{Cl}_{2}(3 \mathrm{x})$. The organic layer was dried over $\mathrm{Na}_{2} \mathrm{SO}_{4}$ filtered and concentrated, purified by silica gel chromatography (EtOAc/petroleum ether, v/v).

\section{2-benzyl- $N$-isopropylbenzenesulfonamide (3a)}

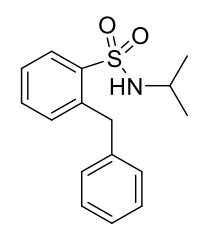

White solid; 0.52 g, $60 \%$ yield; m.p. $43.5-44.6{ }^{\circ} \mathrm{C}$, eluent: EA/PE $=1: 12 ;{ }^{1} \mathrm{H}$ NMR $(400$ $\left.\mathrm{MHz}, \mathrm{CDCl}_{3}\right) \delta 8.03(\mathrm{dd}, J=8.0,1.2 \mathrm{~Hz}, 1 \mathrm{H}), 7.51(\mathrm{td}, J=7.6,1.2 \mathrm{~Hz}, 1 \mathrm{H}), 7.39-$ $7.30(\mathrm{~m}, 4 \mathrm{H}), 7.24-7.22(\mathrm{~m}, 3 \mathrm{H}), 4.46(\mathrm{~s}, 2 \mathrm{H}), 3.53$ (brs, $1 \mathrm{H}), 3.32-3.21(\mathrm{~m}, 1 \mathrm{H}), 0.76$ $(\mathrm{d}, J=6.8 \mathrm{~Hz}, 6 \mathrm{H}) ;{ }^{13} \mathrm{C} \mathrm{NMR}\left(100 \mathrm{MHz}, \mathrm{CDCl}_{3}\right) \delta 139.6,139.04,138.99,133.0,132.5$, 129.8, 129.3, 128.8, 126.8, 126.7, 45.8, 38.8, 23.3; HRMS (ESI-TOF) m/z: $[\mathrm{M}+\mathrm{Na}]^{+}$ Calcd for $\mathrm{C}_{16} \mathrm{H}_{19} \mathrm{NO}_{2} \mathrm{SNa} 312.1029$; Found 312.1026.

2-benzyl- $N$-(tert-butyl)benzenesulfonamide (3b) ${ }^{11}$<smiles>CC(C)(C)NS(=O)(=O)c1ccccc1Cc1ccccc1</smiles> 
White solid; 0.48 g, $53 \%$ yield; m.p. $118.4-118.9{ }^{\circ} \mathrm{C}$, eluent: EA/PE $=1: 12 ;{ }^{1} \mathrm{H}$ NMR $\left(400 \mathrm{MHz}, \mathrm{CDCl}_{3}\right) \delta 8.08(\mathrm{dd}, J=7.6,1.2 \mathrm{~Hz}, 1 \mathrm{H}), 7.48(\mathrm{td}, J=7.2,1.2 \mathrm{~Hz}, 1 \mathrm{H}), 7.37$ $-7.30(\mathrm{~m}, 3 \mathrm{H}), 7.28-7.27(\mathrm{~m}, 1 \mathrm{H}), 7.26-7.18(\mathrm{~m}, 3 \mathrm{H}), 7.26-7.19(\mathrm{~m}, 3 \mathrm{H}), 4.47(\mathrm{~s}$, 2H), 3.93 (brs, 1H), 0.99 (s, 9H); ${ }^{13} \mathrm{C} \mathrm{NMR}\left(100 \mathrm{MHz}, \mathrm{CDCl}_{3}\right) \delta$ 141.3, 139.7, 138.7, 132.8, 132.2, 129.6, 129.1, 128.9, 126.70, 126.67, 54.7, 38.5, 29.9; HRMS (ESI-TOF) m/z: $[\mathrm{M}+\mathrm{Na}]^{+}$Calcd for $\mathrm{C}_{17} \mathrm{H}_{21} \mathrm{NO}_{2} \mathrm{SNa} 326.1185$; Found 326.1179 .

\section{2-benzyl- $N$-methylbenzenesulfonamide $(3 \mathrm{c})^{14}$}

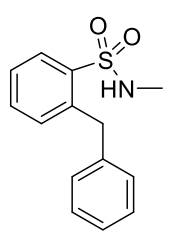

White solid; 0.55 g, $70 \%$ yield; m.p. $72.1-72.5{ }^{\circ} \mathrm{C}$, eluent: EA/PE $=1: 12 ;{ }^{1} \mathrm{H}$ NMR $(400$ $\left.\mathrm{MHz}, \mathrm{CDCl}_{3}\right) \delta 8.02(\mathrm{dd}, J=7.6,1.2 \mathrm{~Hz}, 1 \mathrm{H}), 7.56(\mathrm{td}, J=7.6,1.2 \mathrm{~Hz}, 1 \mathrm{H}), 7.43-7.38$ (m, 2H), 7.33-7.29 (m, 2H), 7.24-7.22 (m, 1H), $7.16(\mathrm{~d}, J=7.6 \mathrm{~Hz}, 2 \mathrm{H}), 4.47(\mathrm{~s}, 2 \mathrm{H})$, $3.34(\mathrm{~d}, J=5.2 \mathrm{~Hz}, 1 \mathrm{H}), 2.18(\mathrm{~d}, J=5.2 \mathrm{~Hz}, 3 \mathrm{H}) ;{ }^{13} \mathrm{C} \mathrm{NMR}\left(150 \mathrm{MHz}, \mathrm{CDCl}_{3}\right) \delta 139.6$, 139.0, 136.3, 133.4, 132.8, 130.9, 128.8, 128.7, 126.81, 126.77, 38.9, 29.1; HRMS (ESI-TOF) m/z: [M+Na $]^{+}$Calcd for $\mathrm{C}_{14} \mathrm{H}_{15} \mathrm{NO}_{2} \mathrm{SNa} 284.0716$; Found 284.0712.

\section{2-(4-(tert-butyl)benzyl)- $N$-isopropylbenzenesulfonamide (3d)}

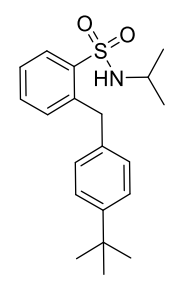

White solid; 0.78 g, 75\% yield; m.p. $100.4-101.3{ }^{\circ} \mathrm{C}$, eluent: $\mathrm{EA} / \mathrm{PE}=1: 12 ;{ }^{1} \mathrm{H}$ NMR $\left(400 \mathrm{MHz}, \mathrm{CDCl}_{3}\right) \delta 8.02(\mathrm{~d}, J=8.0,1.2 \mathrm{~Hz}, 1 \mathrm{H}), 7.53(\mathrm{td}, J=7.6,1.2 \mathrm{~Hz} 1 \mathrm{H}), 7.39-$ 7.32 (m, 4H), 7.18 - $7.16(\mathrm{~m}, 2 \mathrm{H}), 4.43$ (s, 2H), 3.44 (d, J= $7.2 \mathrm{~Hz}, 1 \mathrm{H}), 3.19$ (m, 1H), $1.29(\mathrm{~s}, 9 \mathrm{H}), 0.68(\mathrm{~d}, J=6.4 \mathrm{~Hz}, 6 \mathrm{H}) ;{ }^{13} \mathrm{C} \mathrm{NMR}\left(100 \mathrm{MHz}, \mathrm{CDCl}_{3}\right) \delta \quad 149.9,139.0$, 138.9, $136.5133 .3,132.6129 .9,128.8,126.8,125.8,45.8,38.5,34.4,31.3,23.2$; HRMS (APCI-TOF) m/z: [M+H] ${ }^{+}$Calcd for $\mathrm{C}_{20} \mathrm{H}_{28} \mathrm{NO}_{2} \mathrm{~S} 346.1835$; Found 346.1847 . $N$-isopropyl-2-(4-methylbenzyl)benzenesulfonamide (3e) 


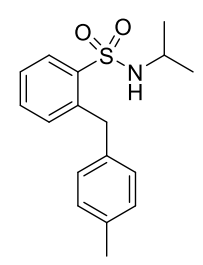

White solid; 0.69 g, $76 \%$ yield; m.p. $89-90{ }^{\circ} \mathrm{C}$, eluent: $\mathrm{EA} / \mathrm{PE}=1: 12 ;{ }^{1} \mathrm{H}$ NMR $(400$ $\left.\mathrm{MHz}, \mathrm{CDCl}_{3}\right) \delta 8.02(\mathrm{dd}, J=8.0,1.2 \mathrm{~Hz}, 1 \mathrm{H}), 7.50(\mathrm{td}, J=7.6,1.2 \mathrm{~Hz}, 1 \mathrm{H}), 7.38-$ $7.32(\mathrm{~m}, 2 \mathrm{H}), 7.14-7.10(\mathrm{~m}, 4 \mathrm{H}), 4.41(\mathrm{~s}, 2 \mathrm{H}), 3.57$ (d, J=8.0 Hz, 1H), $3.32-3.20$ $(\mathrm{m}, 1 \mathrm{H}), 2.32(\mathrm{~s}, 3 \mathrm{H}), 0.76(\mathrm{~d}, J=6.4 \mathrm{~Hz}, 6 \mathrm{H}) ;{ }^{13} \mathrm{C} \mathrm{NMR}\left(100 \mathrm{MHz}, \mathrm{CDCl}_{3}\right) \delta 139.3$, $138.9,136.4,136.4,133.0,132.5,129.8,129.5,129.2,126.7,45.8,38.5,23.2,20.9$; HRMS (ESI-TOF) m/z: [M+Na] ${ }^{+}$Calcd for $\mathrm{C}_{17} \mathrm{H}_{21} \mathrm{NO}_{2} \mathrm{SNa} 326.1185$; Found 326.1175. $\mathrm{N}$-isopropyl-2-(4-(trifluoromethoxy)benzyl)benzenesulfonamide (3f)

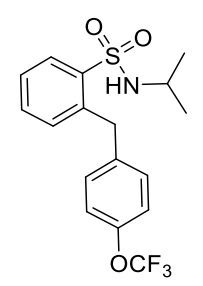

White solid; 0.56 g, $50 \%$ yield; m.p. $79.2-79.4{ }^{\circ} \mathrm{C}$, eluent: $\mathrm{EA} / \mathrm{PE}=1: 12 ;{ }^{1} \mathrm{H} \mathrm{NMR}(600$ MHz, Acetone-d $\left.{ }_{6}\right) \delta 8.04(\mathrm{dd}, J=8.0,1.2 \mathrm{~Hz}, 1 \mathrm{H}), 7.54(\mathrm{td}, J=7.8,1.2 \mathrm{~Hz}, 1 \mathrm{H}), 7.43$ $(\mathrm{td}, J=7.8,1.2 \mathrm{~Hz}, 1 \mathrm{H}), 7.40-7.39(\mathrm{~m}, 2 \mathrm{H}), 7.28-7.24(\mathrm{~m}, 3 \mathrm{H}), 6.36(\mathrm{~d}, J=7.2 \mathrm{~Hz}, 1 \mathrm{H})$, $4.50(\mathrm{~s}, 2 \mathrm{H}), 3.43-3.37(\mathrm{~m}, 1 \mathrm{H}), 1.05(\mathrm{~d}, J=6.6 \mathrm{~Hz}, 6 \mathrm{H}) ;{ }^{13} \mathrm{C} \mathrm{NMR}\left(100 \mathrm{MHz}, \mathrm{CDCl}_{3}\right)$ $\delta 148.0,147.9,139.0,138.5,138.4,132.8,132.7,130.6,129.8,127.0,121.3,46.0,37.9$, 23.4; ${ }^{19} \mathrm{~F} \mathrm{NMR}\left(375 \mathrm{MHz}, \mathrm{CDCl}_{3}\right) \delta-57.95 ; \mathrm{HRMS}(\mathrm{ESI}-\mathrm{TOF}) \mathrm{m} / \mathrm{z}:[\mathrm{M}+\mathrm{Na}]^{+} \mathrm{Calcd}$ for $\mathrm{C}_{17} \mathrm{H}_{18} \mathrm{~F}_{3} \mathrm{NO}_{3} \mathrm{SNa}$ 396.0852; Found 396.0848.

\section{$N$-isopropyl-2-(2-methylbenzyl)benzenesulfonamide (3g)}

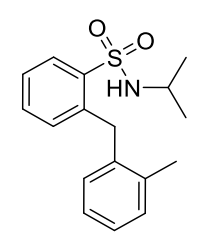

White solid; $0.39 \mathrm{~g}, 43 \%$ yield; m.p. $108.5-108.9^{\circ} \mathrm{C}$, eluent: $\mathrm{EA} / \mathrm{PE}=1: 12$; the product is purified by crystallization from EtOAc/ petroleum ether; ${ }^{1} \mathrm{H} \mathrm{NMR}\left(400 \mathrm{MHz}, \mathrm{CDCl}_{3}\right)$ $\delta 8.06(\mathrm{dd}, J=8.0,1.6 \mathrm{~Hz}, 1 \mathrm{H}), 7.43(\mathrm{td}, J=7.2,1.2 \mathrm{~Hz}, 1 \mathrm{H}), 7.36-7.32(\mathrm{~m}, 1 \mathrm{H}), 7.23$ 
- $7.17(\mathrm{~m}, 2 \mathrm{H}), 7.13-7.12(\mathrm{~m}, 1 \mathrm{H}), 7.03(\mathrm{~d}, J=7.6 \mathrm{~Hz}, 1 \mathrm{H}), 6.99(\mathrm{~d}, J=7.2 \mathrm{~Hz}, 1 \mathrm{H})$, $4.43(\mathrm{~s}, 2 \mathrm{H}), 3.93(\mathrm{~d}, J=7.2 \mathrm{~Hz}, 1 \mathrm{H}), 3.51-3.39(\mathrm{~m}, 1 \mathrm{H}), 2.25(\mathrm{~s}, 3 \mathrm{H}), 0.99$ (d, $J=6.6$ $\mathrm{Hz}, 6 \mathrm{H}) ;{ }^{13} \mathrm{C} \mathrm{NMR}\left(100 \mathrm{MHz}, \mathrm{CDCl}_{3}\right) \delta 139.1,138.9,137.3,137.2,132.6,131.5,130.6$, 130.1, 129.6, 126.9, 126.5, 126.2, 46.1, 36.2, 23.6, 19.7; HRMS (ESI-TOF) m/z: $[\mathrm{M}+\mathrm{Na}]^{+}$Calcd for $\mathrm{C}_{17} \mathrm{H}_{21} \mathrm{NO}_{2} \mathrm{SNa}$ 326.1185; Found 326.1180.

\section{2-(3,5-dimethylbenzyl)- $N$-isopropylbenzenesulfonamide (3h)}

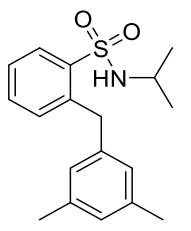

White solid; 0.76 g, $80 \%$ yield; m.p. 82.4-83. ${ }^{\circ} \mathrm{C}$, eluent: EA/PE $=1: 12 ;{ }^{1} \mathrm{H}$ NMR $(400$ $\left.\mathrm{MHz}, \mathrm{CDCl}_{3}\right) \delta=8.03(\mathrm{dd}, J=7.6,1.2 \mathrm{~Hz}, 1 \mathrm{H}), 7.52(\mathrm{td}, J=7.6,1.6 \mathrm{~Hz}, 1 \mathrm{H}), 7.36(\mathrm{~m}$, 2H), $6.88(\mathrm{~s}, 1 \mathrm{H}), 6.83(\mathrm{~s}, 1 \mathrm{H}), 4.38(\mathrm{~s}, 2 \mathrm{H}), 3.59(\mathrm{~d}, J=6.8 \mathrm{~Hz}, 1 \mathrm{H}), 3.31-3.19(\mathrm{~m}$, 1H), $2.26(\mathrm{~s}, 6 \mathrm{H}), 0.73(\mathrm{~d}, J=6.4 \mathrm{~Hz}, 6 \mathrm{H}) ;{ }^{13} \mathrm{C} \mathrm{NMR}\left(100 \mathrm{MHz}, \mathrm{CDCl}_{3}\right) \delta=139.4$, 139.04,139.03 138.5, 133.2, 132.5, 129.8, 128.3, 127.0, 126.7, 45.8, 38.8, 23.1, 21.2; HRMS (APCI-TOF) m/z: [M+H] ${ }^{+}$Calcd for $\mathrm{C}_{18} \mathrm{H}_{24} \mathrm{NO}_{2} \mathrm{~S} 318.1522$; Found 318.1534. 2-(2,6-dimethylbenzyl)- $N$-isopropylbenzenesulfonamide (3i)

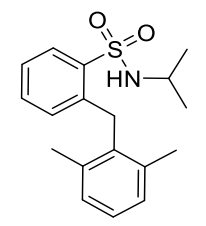

White solid; 0.49 g, 51\% yield; m.p. $154.3-155.2{ }^{\circ} \mathrm{C}$, eluent: $\mathrm{EA} / \mathrm{PE}=1: 12 ;{ }^{1} \mathrm{H}$ NMR $\left(400 \mathrm{MHz}, \mathrm{CDCl}_{3}\right) \delta 8.10-8.07(\mathrm{~m}, 1 \mathrm{H}), 7.35-7.26(\mathrm{~m}, 2 \mathrm{H}), 7.16-7.08(\mathrm{~m}, 3 \mathrm{H}), 6.70$ $-6.67(\mathrm{~m}, 1 \mathrm{H}), 4.46(\mathrm{~d}, J=8.4 \mathrm{~Hz}, 1 \mathrm{H}), 4.44(\mathrm{~s}, 2 \mathrm{H}), 3.64-3.54(\mathrm{~m}, 1 \mathrm{H}), 2.17(\mathrm{~s}, 6 \mathrm{H})$, $1.16(\mathrm{~d}, J=6.4 \mathrm{~Hz}, 6 \mathrm{H}) ;{ }^{13} \mathrm{C} \mathrm{NMR}\left(100 \mathrm{MHz}, \mathrm{CDCl}_{3}\right) \delta 138.6,137.5,135.2,132.9$, 129.6, 128.5, 128.2, 126.8, 126.2, 46.1, 32.0, 23.9, 20.0; HRMS (ESI-TOF) m/z: $[\mathrm{M}+\mathrm{Na}]^{+}$Calcd for $\mathrm{C}_{18} \mathrm{H}_{23} \mathrm{NO}_{2} \mathrm{SNa} 340.1342$; Found 340.1332 .

\section{2-(4-bromobenzyl)- $N$-isopropylbenzenesulfonamide (3j)}




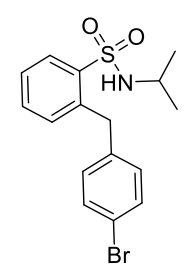

White solid; 0.83 g, $75 \%$ yield; m.p. $96-97{ }^{\circ} \mathrm{C}$, eluent: EA/PE $=1: 12 ;{ }^{1} \mathrm{H}$ NMR $(400$ $\left.\mathrm{MHz}, \mathrm{CDCl}_{3}\right) \delta 8.03(\mathrm{dd}, J=8.0,1.2 \mathrm{~Hz}, 1 \mathrm{H}), 7.49(\mathrm{td}, J=7.6,1.2 \mathrm{~Hz}, 1 \mathrm{H}), 7.45-$ $7.42(\mathrm{~m}, 2 \mathrm{H}), 7.37(\mathrm{td}, J=7.6,0.8 \mathrm{~Hz}, 1 \mathrm{H}), 7.25-7.23(\mathrm{~m}, 1 \mathrm{H}), 7.12-7.09(\mathrm{~m}, 2 \mathrm{H})$, 4.39 (s, 2H), $3.77(\mathrm{~d}, J=7.2 \mathrm{~Hz}, 1 \mathrm{H}), 3.40-3.29(\mathrm{~m}, 1 \mathrm{H}), 0.89(\mathrm{~d}, J=6.4 \mathrm{~Hz}, 6 \mathrm{H})$; ${ }^{13} \mathrm{C} \mathrm{NMR}\left(100 \mathrm{MHz}, \mathrm{CDCl}_{3}\right) \delta 139.0,138.63,138.60,132.7,132.6,131.8,131.0,129.8$, 126.9, 120.5, 46.0, 38.0, 23.5; HRMS (ESI-TOF) m/z: $[\mathrm{M}+\mathrm{Na}]^{+}$Calcd for $\mathrm{C}_{16} \mathrm{H}_{18} \mathrm{BrNO}_{2} \mathrm{SNa} 390.0134$; Found 390.0134.

\section{2-(4-chlorobenzyl)- $N$-isopropylbenzenesulfonamide (3k)}

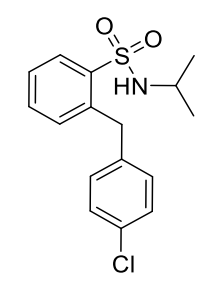

White solid; 0.72 g, $74 \%$ yield; m.p. $94-95{ }^{\circ} \mathrm{C}$, eluent: EA/PE $=1: 12 ;{ }^{1} \mathrm{H}$ NMR $(400$ $\left.\mathrm{MHz}, \mathrm{CDCl}_{3}\right) \delta 8.04(\mathrm{dd}, J=8.0,1.2 \mathrm{~Hz}, 1 \mathrm{H}), 7.50(\mathrm{td}, J=7.6,1.2 \mathrm{~Hz}, 1 \mathrm{H}), 7.37(\mathrm{td}$, $J=8.0,1.2 \mathrm{~Hz}, 1 \mathrm{H}), 7.30-7.27(\mathrm{~m}, 2 \mathrm{H}), 7.25-7.24(\mathrm{~m}, 1 \mathrm{H}), 7.17-7.15(\mathrm{~m}, 2 \mathrm{H}), 4.41$ $(\mathrm{s}, 2 \mathrm{H}), 3.73(\mathrm{~d}, J=7.2 \mathrm{~Hz}, 1 \mathrm{H}), 3.40-3.29(\mathrm{~m}, 1 \mathrm{H}), 0.88(\mathrm{~d}, J=6.4 \mathrm{~Hz}, 6 \mathrm{H}) ;{ }^{13} \mathrm{C}$ NMR $\left(100 \mathrm{MHz}, \mathrm{CDCl}_{3}\right) \delta 139.0,138.7,138.1,132.7,132.6,132.5,130.6,129.8,128.8$, 126.9,46.0, 37.9, 23.5; HRMS (ESI-TOF) $\mathrm{m} / \mathrm{z}:[\mathrm{M}+\mathrm{Na}]^{+} \mathrm{Calcd}$ for $\mathrm{C}_{16} \mathrm{H}_{18} \mathrm{ClNO}_{2} \mathrm{SNa}$ 346.0639; Found 346.0637.

\section{2-(4-fluorobenzyl)- $N$-isopropylbenzenesulfonamide (3I)}<smiles>CC(C)N(Cc1ccc(F)cc1)c1ccccc1C(=O)O</smiles>

White solid; 0.73 g, $79 \%$ yield; m.p. $66.3-66.9{ }^{\circ} \mathrm{C}$, eluent: EA/PE $=1: 12 ;{ }^{1} \mathrm{H}$ NMR $(400$ MHz, Acetone- $\left.\mathrm{d}_{6}\right) \delta 8.04-8.02(\mathrm{~m}, 1 \mathrm{H}), 7.54-7.50(\mathrm{~m}, 1 \mathrm{H}), 7.43-7.39(\mathrm{~m}, 1 \mathrm{H}), 7.32$ $-7.28(\mathrm{~m}, 2 \mathrm{H}), 7.22-7.20(\mathrm{~m}, 1 \mathrm{H}), 7.10-7.06(\mathrm{~m}, 2 \mathrm{H}), 6.31(\mathrm{~d}, J=7.2 \mathrm{~Hz}, 1 \mathrm{H}), 4.44$ 
(s, 2H), $3.48-3.32(\mathrm{~m}, 1 \mathrm{H}), 1.05(\mathrm{~d}, J=6.8 \mathrm{~Hz}, 6 \mathrm{H}) ;{ }^{13} \mathrm{C} \mathrm{NMR}\left(100 \mathrm{MHz}, \mathrm{CDCl}_{3}\right) \delta$ $161.7(J=244.1 \mathrm{~Hz}), 139.0,138.9,135.2(J=3.3 \mathrm{~Hz}), 132.6(J=1.5 \mathrm{~Hz}), 130.8,130.7$, $129.8,126.8,115.6(J=21.1 \mathrm{~Hz}), 46.0,37.8,23.5 ;{ }^{19} \mathrm{~F}$ NMR (375 MHz, Acetone-d 6$)$ $\delta$-118.5; HRMS (ESI-TOF) m/z: $[\mathrm{M}+\mathrm{Na}]^{+}$Calcd for $\mathrm{C}_{16} \mathrm{H}_{18} \mathrm{FNO}_{2} \mathrm{SNa} 330.0934$; Found 330.0942.

\section{2-(2-bromobenzyl)- $N$-isopropylbenzenesulfonamide (3m)}

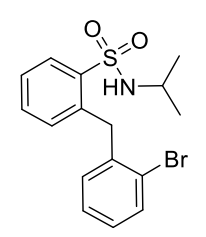

White solid; $0.25 \mathrm{~g}, 23 \%$ yield; m.p. $138.4-138.5^{\circ} \mathrm{C}$, eluent: $\mathrm{EA} / \mathrm{PE}=1: 12$; the product is purified by crystallization from EtOAc/ petroleum ether; ${ }^{1} \mathrm{H}$ NMR (400 MHz, Acetone-d $\left.{ }_{6}\right) \delta 8.06(\mathrm{dd}, J=8.0,1.2 \mathrm{~Hz}, 1 \mathrm{H}), 7.68(\mathrm{dd}, J=7.6,1.2 \mathrm{~Hz}, 1 \mathrm{H}), 7.49(\mathrm{td}, J$ $=7.6,1.6 \mathrm{~Hz}, 1 \mathrm{H}), 7.43(\mathrm{td}, J=7.6,1.2 \mathrm{~Hz}, 1 \mathrm{H}), 7.37(\mathrm{td}, J=7.6,1.2 \mathrm{~Hz}, 1 \mathrm{H}), 7.27-$ $7.21(\mathrm{~m}, 2 \mathrm{H}), 6.91(\mathrm{~d}, J=7.6 \mathrm{~Hz}, 1 \mathrm{H}), 6.52(\mathrm{~d}, J=8.0 \mathrm{~Hz}, 1 \mathrm{H}), 4.61(\mathrm{~s}, 2 \mathrm{H}), 3.51-$ $3.43(\mathrm{~m}, 1 \mathrm{H}), 1.11(\mathrm{~d}, J=6.8 \mathrm{~Hz}, 6 \mathrm{H}) ;{ }^{13} \mathrm{C} \mathrm{NMR}\left(100 \mathrm{MHz}, \mathrm{CDCl}_{3}\right) \delta 138.8,138.6$, 138.2, 133.0, 132.7, 131.7, 131.0, 129.7, 128.4, 127.8, 126.6, 125.3, 46.2, 38.6, 23.8; HRMS (ESI-TOF) m/z: $[\mathrm{M}+\mathrm{Na}]^{+}$Calcd for $\mathrm{C}_{16} \mathrm{H}_{18} \mathrm{BrNO}_{2} \mathrm{SNa}$ 390.0134; Found 390.0139 .

\section{2-(2-bromo-5-fluorobenzyl)- $N$-isopropylbenzenesulfonamide (3n)}

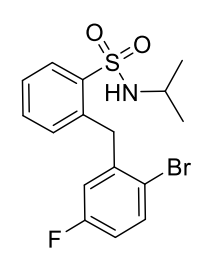

White solid; 0.46 g, $40 \%$ yield; m.p.121.9-122.4 ${ }^{\circ} \mathrm{C}$, eluent: $\mathrm{EA} / \mathrm{PE}=1: 12$; the product is purified by crystallization from EtOAc/ petroleum ether; ${ }^{1} \mathrm{H} \mathrm{NMR}\left(400 \mathrm{MHz}, \mathrm{CDCl}_{3}\right)$ $\delta 8.09(\mathrm{dd}, J=8.0,1.2 \mathrm{~Hz}, 1 \mathrm{H}), 7.57(\mathrm{dd}, J=8.8,5.2 \mathrm{~Hz}, 1 \mathrm{H}), 7.47(\mathrm{td}, J=7.6,1.2 \mathrm{~Hz}$, 1H), $7.39(\mathrm{td}, J=7.6,1.2 \mathrm{~Hz}, 1 \mathrm{H}), 6.99$ (d, $J=7.6 \mathrm{~Hz}, 1 \mathrm{H}), 6.90-6.86(\mathrm{~m}, 1 \mathrm{H}), 6.80$ $(\mathrm{dd}, J=9.2,2.8 \mathrm{~Hz}, 1 \mathrm{H}), 4.53(\mathrm{~s}, 2 \mathrm{H}), 4.26(\mathrm{~d}, J=7.2 \mathrm{~Hz}, 1 \mathrm{H}), 3.58-3.46(\mathrm{~m}, 1 \mathrm{H})$, $1.08(\mathrm{~d}, J=6.4 \mathrm{~Hz}, 6 \mathrm{H}) ;{ }^{13} \mathrm{C} \mathrm{NMR}\left(100 \mathrm{MHz}, \mathrm{CDCl}_{3}\right) \delta 163.1(J=245.8 \mathrm{~Hz}), 141.0$ 
$(J=7.4 \mathrm{~Hz}), 139.0,137.4,134.0(J=8.0 \mathrm{~Hz}), 132.8,131.1,129.7,126.9,119.2(J=3.2$ $\mathrm{Hz}), 118.4(J=22.9 \mathrm{~Hz}), 115.6(J=22.4 \mathrm{~Hz}), 46.2,38.6,23.8 ;{ }^{19} \mathrm{~F}$ NMR $(375 \mathrm{MHz}$, $\left.\mathrm{CDCl}_{3}\right) \delta$-114.3; HRMS (ESI-TOF) $\mathrm{m} / \mathrm{z}:[\mathrm{M}+\mathrm{H}]^{+}$Calcd for $\mathrm{C}_{16} \mathrm{H}_{18} \mathrm{BrFNO}_{2} \mathrm{~S}$ 386.0220; Found 386.0232.

\section{2-(3-chlorobenzyl)- $N$-isopropylbenzenesulfonamide (3o)}

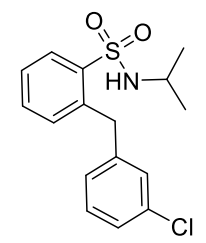

White solid; 0.24 g, $25 \%$ yield; m.p. $73-74{ }^{\circ} \mathrm{C}$, eluent: $\mathrm{EA} / \mathrm{PE}=1: 12$; the product is purified by crystallization from EtOAc/ petroleum ether; ${ }^{1} \mathrm{H}$ NMR $(600 \mathrm{MHz}$, Acetone$\left.\mathrm{d}_{6}\right) \delta 8.04(\mathrm{dd}, J=7.8,1.2 \mathrm{~Hz}, 1 \mathrm{H}), 7.54(\mathrm{dd}, J=7.8,1.2 \mathrm{~Hz}, 1 \mathrm{H}), 7.43(\mathrm{t}, J=7.8 \mathrm{~Hz}$, 1H), $7.34(\mathrm{t}, J=7.8 \mathrm{~Hz}, 1 \mathrm{H}), 7.30(\mathrm{~s}, 1 \mathrm{H}), 7.27-7.23(\mathrm{~m}, 3 \mathrm{H}), 6.37(\mathrm{~d}, J=7.2 \mathrm{~Hz}, 1 \mathrm{H})$, $4.47(\mathrm{~s}, 2 \mathrm{H}), 3.42(\mathrm{~m}, 1 \mathrm{H}), 1.06(\mathrm{~d}, J=6.6 \mathrm{~Hz}, 6 \mathrm{H}) ;{ }^{13} \mathrm{C} \mathrm{NMR}\left(100 \mathrm{MHz}, \mathrm{CDCl}_{3}\right) \delta$ $141.7,139.1,138.3,134.6,132.8,132.7,130.0,129.8,129.2,127.6,127.0,126.8,46.0$, 38.2, 23.4; HRMS (ESI-TOF) m/z: $[\mathrm{M}+\mathrm{Na}]^{+}$Calcd for $\mathrm{C}_{16} \mathrm{H}_{18} \mathrm{ClNO}_{2} \mathrm{SNa} 346.0639$; Found 346.0648 .

\section{2-(3-cyanobenzyl)- $N$-isopropylbenzenesulfonamide (3p)}

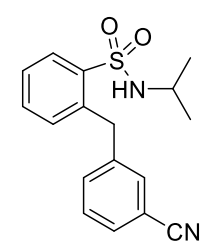

White solid; 0.2 g, $21 \%$ yield; m.p. $111.0-111.3{ }^{\circ} \mathrm{C}$, eluent: $\mathrm{EA} / \mathrm{PE}=1: 6$; the product is purified by crystallization from EtOAc/ petroleum ether; ${ }^{1} \mathrm{H} \mathrm{NMR}\left(600 \mathrm{MHz}, \mathrm{CDCl}_{3}\right)$ $\delta 8.06(\mathrm{dd}, J=7.8,1.2 \mathrm{~Hz}, 1 \mathrm{H}), 7.54-7.49(\mathrm{~m}, 3 \mathrm{H}), 7.45-7.40(\mathrm{~m}, 3 \mathrm{H}), 7.20(\mathrm{~d}, J=7.8$ $\mathrm{Hz}, 1 \mathrm{H}), 4.48(\mathrm{~s}, 2 \mathrm{H}), 3.87(\mathrm{~d}, J=7.2 \mathrm{~Hz}, 1 \mathrm{H}), 3.46-3.38(\mathrm{~m}, 1 \mathrm{H}), 0.97(\mathrm{~d}, J=6.6 \mathrm{~Hz}$, $6 \mathrm{H}) ;{ }^{13} \mathrm{C}$ NMR $\left(100 \mathrm{MHz}, \mathrm{CDCl}_{3}\right) \delta 141.3,139.2,137.8,133.8,132.8,132.6,132.5$, 130.2, 129.7, 129.4, 127.2, 118.6, 112.6, 46.1, 37.8, 23.6; HRMS (ESI-TOF) m/z: $[\mathrm{M}+\mathrm{Na}]^{+}$Calcd for $\mathrm{C}_{17} \mathrm{H}_{18} \mathrm{~N}_{2} \mathrm{O}_{2} \mathrm{SNa}$ 337.0981; Found 337.0989.

\section{2-benzyl- $N$-isopropyl-4-methylbenzenesulfonamide (3q)}




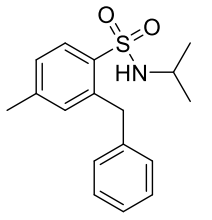

White solid; 0.56 g, $61 \%$ yield; m.p. $74-75{ }^{\circ} \mathrm{C}$, eluent: EA/PE $=1: 14 ;{ }^{1} \mathrm{H}$ NMR $(400$ $\left.\mathrm{MHz}, \mathrm{CDCl}_{3}\right) \delta=7.90(\mathrm{~d}, J=8.0 \mathrm{~Hz}, 1 \mathrm{H}), 7.36-7.30(\mathrm{~m}, 2 \mathrm{H}), 7.24-7.23(\mathrm{~m}, 3 \mathrm{H})$, $7.17-7.14(\mathrm{~m}, 2 \mathrm{H}), 4.41(\mathrm{~s}, 2 \mathrm{H}), 3.47$ (d, $J=7.2 \mathrm{~Hz}, 1 \mathrm{H}), 3.29-3.17(\mathrm{~m}, 1 \mathrm{H}), 2.40$ $(\mathrm{s}, 3 \mathrm{H}), 0.74(\mathrm{~d}, J=6.4 \mathrm{~Hz}, 6 \mathrm{H}) ;{ }^{13} \mathrm{C} \mathrm{NMR}\left(100 \mathrm{MHz}, \mathrm{CDCl}_{3}\right) \delta=143.2,139.7,138.8$, 133.8, 130.1, 129.2, 128.8, 127.4, 126.7, 45.7, 38.8, 23.3, 21.3; HRMS (ESI-TOF) m/z: $[\mathrm{M}+\mathrm{Na}]^{+}$Calcd for $\mathrm{C}_{17} \mathrm{H}_{21} \mathrm{NO}_{2} \mathrm{SNa} 326.1185$; Found 326.1189 .

\section{2-benzyl- $N$-isopropyl-5-methylbenzenesulfonamide (3r)}<smiles>Cc1ccc(Cc2ccccc2)c(S(=O)(=O)NC(C)C)c1</smiles>

White solid; 0.58 g, 64\% yield; m.p. $64.4-65.3{ }^{\circ} \mathrm{C}$, eluent: EA/PE $=1: 14 ;{ }^{1} \mathrm{H}$ NMR $(400$ $\left.\mathrm{MHz}, \mathrm{CDCl}_{3}\right) \delta 7.84(\mathrm{~s}, 1 \mathrm{H}), 7.32-7.30(\mathrm{~m}, 3 \mathrm{H}), 7.25$ - $7.22(\mathrm{~m}, 4 \mathrm{H}), 4.41(\mathrm{~s}, 2 \mathrm{H}), 3.48$ $(\mathrm{d}, J=6.8 \mathrm{~Hz}, 1 \mathrm{H}), 3.27-3.22(\mathrm{~m}, 1 \mathrm{H}), 2.40(\mathrm{~s}, 3 \mathrm{H}), 0.75(\mathrm{~d}, J=6.8 \mathrm{~Hz}, 6 \mathrm{H}) ;{ }^{13} \mathrm{C} \mathrm{NMR}$ $\left(100 \mathrm{MHz}, \mathrm{CDCl}_{3}\right) \delta 139.9,138.6,136.8,135.8,133.2,133.0,130.2,129.2,128.8$, 126.6, 45.8, 38.4, 23.3, 20.9; HRMS (ESI-TOF) $\mathrm{m} / \mathrm{z}:[\mathrm{M}+\mathrm{Na}]^{+} \mathrm{Calcd}$ for $\mathrm{C}_{17} \mathrm{H}_{21} \mathrm{NO}_{2} \mathrm{SNa}$ 326.1185; Found 326.1190.

\section{2-benzyl-4-chloro- $N$-isopropylbenzenesulfonamide (3s)}<smiles>CC(C)N[SH](=O)(O)c1ccc(Cl)cc1Cc1ccccc1</smiles>

White solid; 0.42 g, $43 \%$ yield; m.p. $69.6-70.2{ }^{\circ} \mathrm{C}$, eluent: $\mathrm{EA} / \mathrm{PE}=1: 14 ;{ }^{1} \mathrm{H}$ NMR $(400$ $\left.\mathrm{MHz}, \mathrm{CDCl}_{3}\right) \delta 7.96(\mathrm{~d}, J=8.4 \mathrm{~Hz}, 1 \mathrm{H}), 7.36-7.32(\mathrm{~m}, 3 \mathrm{H}), 7.29-7.27(\mathrm{~m}, 2 \mathrm{H}), 7.24$ $-7.21(\mathrm{~m}, 2 \mathrm{H}), 4.41(\mathrm{~s}, 2 \mathrm{H}), 3.66(\mathrm{~d}, J=7.2 \mathrm{~Hz}, 1 \mathrm{H}), 3.33-3.21(\mathrm{~m}, 1 \mathrm{H}), 0.79(\mathrm{~d}, J=$ $6.4 \mathrm{~Hz}, 6 \mathrm{H}) ;{ }^{13} \mathrm{C} \mathrm{NMR}\left(100 \mathrm{MHz}, \mathrm{CDCl}_{3}\right) \delta 141.1,138.7,138.6,137.6,132.7,131.3$, 129.3 129.0, 127.0, 126.9, 46.0, 38.6, 23.3; HRMS (ESI-TOF) m/z: [M+Na] $]^{+} \mathrm{Calcd}$ for 
$\mathrm{C}_{16} \mathrm{H}_{18} \mathrm{ClNO}_{2} \mathrm{SNa} 346.0639$; Found 346.0645.

2-benzyl-3-chloro- $N$-isopropylbenzenesulfonamide (3t)

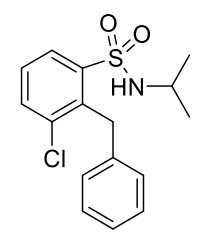

White solid; 0.57 g, 59\% yield; m.p. $112.8-113.2{ }^{\circ} \mathrm{C}$ eluent: EA/PE $=1: 14 ;{ }^{1} \mathrm{H}$ NMR $\left(600 \mathrm{MHz}, \mathrm{CDCl}_{3}\right) \delta 8.02(\mathrm{dd}, J=7.8,1.2 \mathrm{~Hz}, 1 \mathrm{H}), 7.69(\mathrm{dd}, J=7.8,1.2 \mathrm{~Hz}, 1 \mathrm{H}), 7.36$ (t, $J=8.4 \mathrm{~Hz}, 1 \mathrm{H}), 7.32-7.30(\mathrm{~m}, 2 \mathrm{H}), 7.25-7.23(\mathrm{~m}, 1 \mathrm{H}), 7.20-7.19(\mathrm{~m}, 2 \mathrm{H}), 4.75$ $(\mathrm{s}, 2 \mathrm{H}), 3.41(\mathrm{~d}, J=6.6 \mathrm{~Hz}, 1 \mathrm{H}), 3.24-3.16(\mathrm{~m}, 1 \mathrm{H}), 0.63(\mathrm{~d}, J=6.0 \mathrm{~Hz}, 6 \mathrm{H}) ;{ }^{13} \mathrm{C}$ NMR $\left(100 \mathrm{MHz}, \mathrm{CDCl}_{3}\right) \delta 141.8,138.2,137.9,136.4,133.9,128.9,128.5,128.4,127.7$, 126.8, 46.0, 34.7, 23.0; HRMS (ESI-TOF) m/z: [M+Na $]^{+} \mathrm{Calcd}$ for $\mathrm{C}_{16} \mathrm{H}_{18} \mathrm{ClNO}_{2} \mathrm{SNa}$ 346.0639; Found 346.0643.

2-benzyl-6-chloro- $N$-isopropylbenzenesulfonamide (3u)

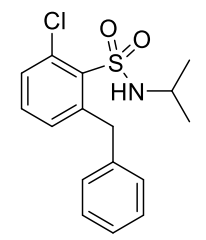

White solid; $0.41 \mathrm{~g}, 42 \%$ yield; m.p. $92.1-93.3{ }^{\circ} \mathrm{C}$, eluent: $\mathrm{EA} / \mathrm{PE}=1: 14 ;{ }^{1} \mathrm{H}$ NMR $(600$ MHz, Acetone-d $\left.\mathrm{d}_{6}\right) \delta 7.56-7.51(\mathrm{~m}, 2 \mathrm{H}), 7.37(\mathrm{dd}, J=7.2,1.2 \mathrm{~Hz}, 1 \mathrm{H}), 7.28$ - $7.25(\mathrm{~m}$, 2H), 7.19 - $7.16(\mathrm{~m}, 3 \mathrm{H}), 6.47$ (d, J=7.2 Hz, 1H), $4.62(\mathrm{~s}, 2 \mathrm{H}), 3.18-3.10(\mathrm{~m}, 1 \mathrm{H})$, $0.96(\mathrm{~d}, J=6.6 \mathrm{~Hz}, 6 \mathrm{H}) ;{ }^{13} \mathrm{C}$ NMR $\left(150 \mathrm{MHz}\right.$, Acetone-d $\left.{ }_{6}\right) \delta 144.8,142.2,139.3,134.6$, 133.9, 133.4, 132.0, 130.1, 129.4, 127.1, 46.9, 41.1, 23.8; HRMS (ESI-TOF) m/z: $[\mathrm{M}+\mathrm{Na}]^{+}$Calcd for $\mathrm{C}_{16} \mathrm{H}_{18} \mathrm{ClNO}_{2} \mathrm{SNa} 346.0639$; Found 346.0645. 


\section{6) Synthesis of five-membered benzoslutams}

\section{General Procedure:}

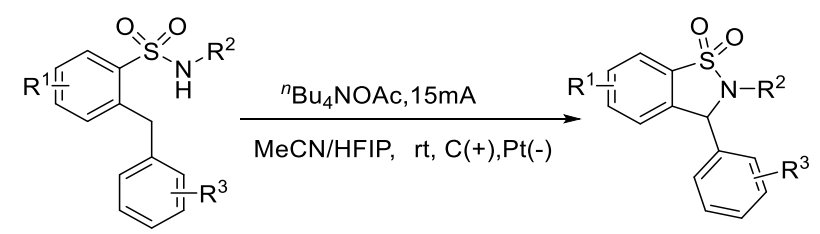

Substrate (0.2 mmol), $n$-Bu 4 NOAc $(0.2 \mathrm{mmol}, 60.3 \mathrm{mg})$ and a mixture of MeOH/HFIP (V/V $4.8 \mathrm{~mL} / 1.2 \mathrm{~mL}$ ) were added to a $10 \mathrm{~mL}$ three-necked round-bottomed flask equipped with carbon rod $(6 \mathrm{~mm})$ as the anode and platinum plate $(1 \mathrm{~cm} \times 1 \mathrm{~cm} \times 0.2$ $\mathrm{mm})$ as the cathode. Under the argon atmosphere, the reaction mixture was stirred and electrolyzed at a constant current of $15 \mathrm{~mA}$ for $1.5 \mathrm{~h}$ at room temperature . Upon completion, the reaction mixture was concentrated under reduced pressure. The residue was chromatographed by silica gel chromatography (EtOAc / petroleum ether or EtOAc / toluene V/V) .

\section{2-isopropyl-3-phenyl-2,3-dihydrobenzo[d]isothiazole 1,1-dioxide (4a) ${ }^{15}$}<smiles>CC(C)N(c1ccccc1)S(=O)(=O)c1ccccc1</smiles>

White solid; $41.5 \mathrm{mg}, 72 \%$ yield; m.p. $131.1-132.6{ }^{\circ} \mathrm{C}$, eluent: EA/PE $=1: 10 ;{ }^{1} \mathrm{H}$ NMR $\left(400 \mathrm{MHz}, \mathrm{CDCl}_{3}\right) \delta 7.82-7.78(\mathrm{~m}, 1 \mathrm{H}), 7.51-7.44(\mathrm{~m}, 2 \mathrm{H}), 7.40-7.31(\mathrm{~m}, 5 \mathrm{H})$, $7.04-7.00$ (m, 1H), 5.54 (s, 1H), 3.94 (hept, $J=6.8 \mathrm{~Hz}, 1 \mathrm{H}), 1.47$ (d, $J=6.8 \mathrm{~Hz}, 3 \mathrm{H})$, $1.13(\mathrm{~d}, J=6.8 \mathrm{~Hz}, 3 \mathrm{H}) ;{ }^{13} \mathrm{C} \mathrm{NMR}\left(100 \mathrm{MHz}, \mathrm{CDCl}_{3}\right) \delta 139.6,138.4,134.3,132.7$, 129.1, 129.0, 128.7, 127.7, 125.0, 120.8, 62.4, 46.6, 21.9, 20.3. HRMS (ESI-TOF) m/z: $[\mathrm{M}+\mathrm{Na}]^{+}$Calcd for $\mathrm{C}_{16} \mathrm{H}_{17} \mathrm{NO}_{2} \mathrm{SNa}$ 310.0872; Found 310.0869.

2-(tert-butyl)-3-phenyl-2,3-dihydrobenzo[d]isothiazole 1,1-dioxide (4b)<smiles>CC(C)(C)N1C(c2ccccc2)c2ccccc2S1(=O)=O</smiles>

White solid; $49.5 \mathrm{mg}, 82 \%$ yield; m.p. $195.0-195.9{ }^{\circ} \mathrm{C}$, eluent: $\mathrm{EA} / \mathrm{PE}=1: 10 ;{ }^{1} \mathrm{H} \mathrm{NMR}$ 
$\left(400 \mathrm{MHz}\right.$, Acetone- $\left.\mathrm{d}_{6}\right) \delta 7.76-7.71(\mathrm{~m}, 1 \mathrm{H}), 7.63-7.60(\mathrm{~m}, 2 \mathrm{H}), 7.58-7.52(\mathrm{~m}, 2 \mathrm{H})$, $7.40-7.35(\mathrm{~m}, 2 \mathrm{H}), 7.31-7.26(\mathrm{~m}, 1 \mathrm{H}), 7.24-7.21(\mathrm{~m}, 1 \mathrm{H}), 6.01(\mathrm{~s}, 1 \mathrm{H}), 1.44(\mathrm{~s}$, $9 \mathrm{H}) ;{ }^{13} \mathrm{C} \mathrm{NMR}\left(100 \mathrm{MHz}, \mathrm{CDCl}_{3}\right) \delta 142.8,137.9,134.4,132.7,129.0,128.9,128.0$, 126.4, 124.8, 120.7, 63.5, 58.6, 29.0; HRMS (ESI-TOF) m/z: $[\mathrm{M}+\mathrm{Na}]^{+}$Calcd for $\mathrm{C}_{17} \mathrm{H}_{19} \mathrm{NO}_{2} \mathrm{~S}$ Na 324.1029; Found 324.1029.

\section{2-methyl-3-phenyl-2,3-dihydrobenzo[d]isothiazole 1,1-dioxide (4c) ${ }^{14}$}

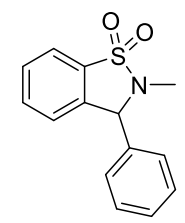

White solid; $10.4 \mathrm{mg}, 20 \%$ yield, eluent: EA/ toluene $=1: 220 ;{ }^{1} \mathrm{H}$ NMR $(600 \mathrm{MHz}$, $\left.\mathrm{CDCl}_{3}\right) \delta 7.86(\mathrm{~d}, J=7.2 \mathrm{~Hz}, 1 \mathrm{H}), 7.54-7.50(\mathrm{~m}, 2 \mathrm{H}), 7.41-7.38(\mathrm{~m}, 3 \mathrm{H}), 7.34-7.33$ $(\mathrm{m}, 2 \mathrm{H}), 7.04(\mathrm{~d}, J=7.2 \mathrm{~Hz}, 1 \mathrm{H}), 5.19(\mathrm{~s}, 1 \mathrm{H}), 2.78(\mathrm{~s}, 3 \mathrm{H}),{ }^{13} \mathrm{C} \mathrm{NMR}\left(150 \mathrm{MHz}, \mathrm{CDCl}_{3}\right)$ $\delta 138.6,136.6,134.0,133.0,129.3,129.2,128.1,125.0,121.1,67.0,27.4$; HRMS (ESITOF) $\mathrm{m} / \mathrm{z}:[\mathrm{M}+\mathrm{Na}]^{+}$Calcd for $\mathrm{C}_{14} \mathrm{H}_{13} \mathrm{NO}_{2} \mathrm{SNa} 282.0559$; Found 282.0568.

\section{3-(4-(tert-butyl)phenyl)-2-isopropyl-2,3-dihydrobenzo[d]isothiazole1,1-dioxide}

(4d)

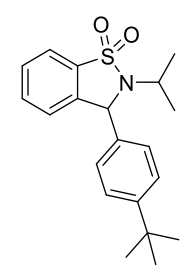

White solid; $55.6 \mathrm{mg}, 81 \%$ yield; m.p. $148-149{ }^{\circ} \mathrm{C}$, eluent: EA/PE $=1: 10 ;{ }^{1} \mathrm{H}$ NMR $\left(600 \mathrm{MHz}, \mathrm{CDCl}_{3}\right) \delta 7.80-7.78(\mathrm{~m}, 1 \mathrm{H}), 7.50-7.45(\mathrm{~m}, 2 \mathrm{H}), 7.37-7.36(\mathrm{~m}, 2 \mathrm{H}), 7.30-$ $7.29(\mathrm{~m}, 2 \mathrm{H}), 7.17-7.16(\mathrm{~m}, 1 \mathrm{H}), 5.52(\mathrm{~s}, 1 \mathrm{H})$, (hept, $J=6.8 \mathrm{~Hz}, 1 \mathrm{H}), 1.48(\mathrm{~d}, J=6.6$ $\mathrm{Hz}, 3 \mathrm{H}), 1.30$ (s, 9H), 1.14 (d, $J=6.6 \mathrm{~Hz}, 3 \mathrm{H}) ;{ }^{13} \mathrm{C} \mathrm{NMR}\left(150 \mathrm{MHz}, \mathrm{CDCl}_{3}\right) \delta 151.8$, $138.5,136.2,134.4,132.6,129.0,127.3,125.9,125.0,120.7,62.2,46.7,34.6,31.3$, 21.7, 20.4; HRMS (ESI-TOF) m/z: $[\mathrm{M}+\mathrm{Na}]^{+}$Calcd for $\mathrm{C}_{20} \mathrm{H}_{25} \mathrm{NO}_{2} \mathrm{SNa}$ 366.1498; Found 366.1505.

\section{2-isopropyl-3-(p-tolyl)-2,3-dihydrobenzo[ $d]$ isothiazole 1,1-dioxide (4e)}




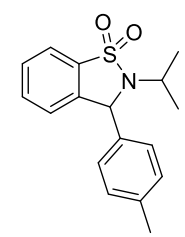

White solid; $51 \mathrm{mg}, 85 \%$ yield; m.p. $127-128{ }^{\circ} \mathrm{C}$, eluent: EA/PE $=1: 10 ;{ }^{1} \mathrm{H}$ NMR $(400$ $\left.\mathrm{MHz}, \mathrm{CDCl}_{3}\right) \delta 7.82-7.77(\mathrm{~m}, 1 \mathrm{H}), 7.51-7.44(\mathrm{~m}, 2 \mathrm{H}), 7.28-7.27(\mathrm{~m}, 1 \mathrm{H}), 7.18-7.16$ (m, 2H), $7.05-6.99(\mathrm{~m}, 1 \mathrm{H}), 5.51(\mathrm{~s}, 1 \mathrm{H}), 3.92$ (hept, $J=6.8 \mathrm{~Hz}, 1 \mathrm{H}), 2.35$ (s, 3H), $1.47(\mathrm{~d}, J=6.8 \mathrm{~Hz}, 3 \mathrm{H}), 1.13(\mathrm{~d}, J=6.8 \mathrm{~Hz}, 3 \mathrm{H}) ;{ }^{13} \mathrm{C}$ NMR $\left(100 \mathrm{MHz}, \mathrm{CDCl}_{3}\right) \delta$ 138.62, 138.58, 136.5, 134.3, 132.7, 129.7, 129.1, 127.6, 124.9, 120.7, 62.2, 46.6, 21.8, 21.2, 20.3; HRMS (ESI-TOF) m/z: $[\mathrm{M}+\mathrm{Na}]^{+}$Calcd for $\mathrm{C}_{17} \mathrm{H}_{19} \mathrm{NO}_{2} \mathrm{SNa}$ 324.1029; Found 324.1034 .

\section{2-isopropyl-3-(4-(trifluoromethoxy)phenyl)-2,3-dihydrobenzo[d]isothiazole1,1-} dioxide (4f)

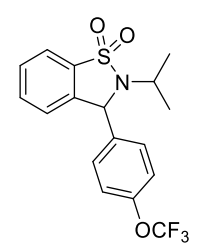

White solid; $63 \mathrm{mg}, 85 \%$ yield; m.p. $79.2-79.4{ }^{\circ} \mathrm{C}$, eluent: $\mathrm{EA} / \mathrm{PE}=1: 10 ;{ }^{1} \mathrm{H}$ NMR $(400$ $\left.\mathrm{MHz}, \mathrm{CDCl}_{3}\right) \delta 7.86-7.79(\mathrm{~m}, 1 \mathrm{H}), 7.54-7.49(\mathrm{~m}, 2 \mathrm{H}), 7.46-7.43(\mathrm{~m}, 2 \mathrm{H}), 7.23-$ $7.21(\mathrm{~m}, 2 \mathrm{H}), 7.04-7.00(\mathrm{~m}, 1 \mathrm{H}), 5.56(\mathrm{~s}, 1 \mathrm{H}), 3.94$ (hept, $J=6.8 \mathrm{~Hz}, 1 \mathrm{H}), 1.47$ (d, $J$ $=6.8 \mathrm{~Hz}, 3 \mathrm{H}), 1.14(\mathrm{~d}, J=6.8 \mathrm{~Hz}, 3 \mathrm{H}) ;{ }^{13} \mathrm{C} \mathrm{NMR}\left(100 \mathrm{MHz}, \mathrm{CDCl}_{3}\right) \delta$ 149.4, 138.4, $137.7,134.3,132.9,129.4,129.1,124.9,121.5,121.0,61.5,46.8,21.9,20.3 ;{ }^{19} \mathrm{~F}$ NMR $\left(375 \mathrm{MHz}, \mathrm{CDCl}_{3}\right) \quad \delta \quad-57.9 ;$ HRMS (ESI-TOF) $\mathrm{m} / \mathrm{z}:[\mathrm{M}+\mathrm{Na}]^{+}$Calcd for $\mathrm{C}_{17} \mathrm{H}_{16} \mathrm{~F}_{3} \mathrm{NO}_{3} \mathrm{SNa}$ 394.0695; Found 394.0704.

\section{2-isopropyl-3-(o-tolyl)-2,3-dihydrobenzo[d]isothiazole 1,1-dioxide (4g)}

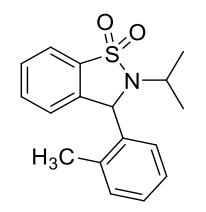

White solid; $45 \mathrm{mg}, 75 \%$ yield; m.p. $144-145^{\circ} \mathrm{C}$, eluent: EA/PE $=1: 10 ;{ }^{1} \mathrm{H}$ NMR $(400$ $\left.\mathrm{MHz}, \mathrm{CDCl}_{3}\right) \delta 7.84-7.79(\mathrm{~m}, 1 \mathrm{H}), 7.52-7.45(\mathrm{~m}, 2 \mathrm{H}), 7.34-7.31(\mathrm{~m}, 1 \mathrm{H}), 7.24-$ $7.27(\mathrm{~m}, 1 \mathrm{H}), 7.21-7.19(\mathrm{~m}, 2 \mathrm{H}), 7.00-6.93(\mathrm{~m}, 1 \mathrm{H}), 5.81(\mathrm{~s}, 1 \mathrm{H}), 3.90$ (hept, $J=6.8$ 
$\mathrm{Hz}, 1 \mathrm{H}), 2.35(\mathrm{~s}, 3 \mathrm{H}), 1.48(\mathrm{~d}, J=6.8 \mathrm{~Hz}, 3 \mathrm{H}), 1.11(\mathrm{~d}, J=6.8 \mathrm{~Hz}, 3 \mathrm{H}) ;{ }^{13} \mathrm{C} \mathrm{NMR}(150$ $\left.\mathrm{MHz}_{2} \mathrm{CDCl}_{3}\right) \delta 138.2,134.3,132.8,131.4,129.1,128.6,126.7,124.4,120.9,46.4,21.4$, 20.4, 19.2; HRMS (APCI-TOF) m/z: [M+H] $]^{+}$Calcd for $\mathrm{C}_{17} \mathrm{H}_{20} \mathrm{NO}_{2} \mathrm{~S} 302.1209$; Found 302.1212 .

\section{3-(3,5-dimethylphenyl)-2-isopropyl-2,3-dihydrobenzo[d]isothiazole1,1-dioxide}

(4h)

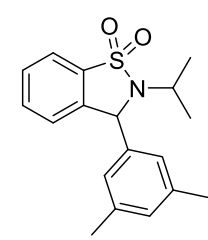

White solid; $52 \mathrm{mg}, 82 \%$ yield; m.p. $70.4-71.2{ }^{\circ} \mathrm{C}$, eluent: $\mathrm{EA} / \mathrm{PE}=1: 10 ;{ }^{1} \mathrm{H}$ NMR $(400$ $\left.\mathrm{MHz}, \mathrm{CDCl}_{3}\right) \delta 7.82-7.77(\mathrm{~m}, 1 \mathrm{H}), 7.52-7.44(\mathrm{~m}, 2 \mathrm{H}), 7.05-7.01(\mathrm{~m}, 1 \mathrm{H}), 6.98(\mathrm{~s}$, 2H), $6.96(\mathrm{~s}, 1 \mathrm{H}), 5.46(\mathrm{~s}, 1 \mathrm{H}), 3.91(\mathrm{hept}, J=6.8 \mathrm{~Hz}, 1 \mathrm{H}), 2.30(\mathrm{~s}, 6 \mathrm{H}), 1.48$ (d, $J=6.8$ $\mathrm{Hz}, 3 \mathrm{H}), 1.16(\mathrm{~d}, J=6.8 \mathrm{~Hz}, 3 \mathrm{H}) ;{ }^{13} \mathrm{C} \mathrm{NMR}\left(100 \mathrm{MHz}, \mathrm{CDCl}_{3}\right) \delta 139.3,138.7,138.5$, 134.2, 132.7, 130.4, 129.0, 125.4, 125.0, 120.7, 62.5, 46.7, 21.7, 21.2 20.4; HRMS (ESI-TOF) m/z: [M+Na] ${ }^{+}$Calcd for $\mathrm{C}_{18} \mathrm{H}_{21} \mathrm{NO}_{2} \mathrm{SNa} 338.1185$; Found 338.1191.

3-(2,6-dimethylphenyl)-2-isopropyl-2,3-dihydrobenzo[d]isothiazole 1,1-dioxide (4i)

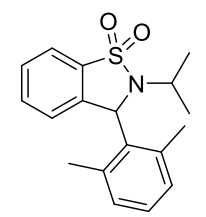

White solid; $52.4 \mathrm{mg}, 83 \%$ yield; m.p. $165.5-166.7{ }^{\circ} \mathrm{C}$, eluent: $\mathrm{EA} / \mathrm{PE}=1: 10 ;{ }^{1} \mathrm{H}$ NMR $\left(400 \mathrm{MHz}, \mathrm{CDCl}_{3}\right) \delta 7.85-7.80(\mathrm{~m}, 1 \mathrm{H}), 7.53-7.47(\mathrm{~m}, 2 \mathrm{H}), 7.18-7.11(\mathrm{~m}, 2 \mathrm{H})$, $6.96-6.92(\mathrm{~m}, 2 \mathrm{H}), 6.09$ (s, 1H), 3.79 (hept, $J=6.8 \mathrm{~Hz}, 1 \mathrm{H}), 2.60(\mathrm{~s}, 3 \mathrm{H}), 1.92(\mathrm{~s}, 3 \mathrm{H})$, $1.50(\mathrm{~d}, J=6.8 \mathrm{~Hz}, 3 \mathrm{H}), 1.18(\mathrm{~d}, J=6.8 \mathrm{~Hz}, 3 \mathrm{H}) ;{ }^{13} \mathrm{C} \mathrm{NMR}\left(100 \mathrm{MHz}, \mathrm{CDCl}_{3}\right) \delta 139.1$, $137.5,137.0,134.9,133.3,132.9,131.1,129.1,128.8,128.6,123.7,120.9,58.0,46.7$, 20.9, 20.7, 20.6, 19.4; HRMS (ESI-TOF) m/z: $[\mathrm{M}+\mathrm{Na}]^{+}$Calcd for $\mathrm{C}_{18} \mathrm{H}_{21} \mathrm{NO}_{2} \mathrm{SNa}$ 338.1185; Found 338.1191.

3-(4-bromophenyl)-2-isopropyl-2,3-dihydrobenzo[d]isothiazole 1,1-dioxide (4j) 


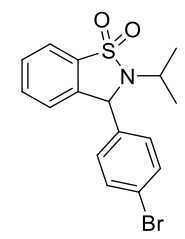

White solid; 66 mg, 90\% yield; m.p. 93.8-94.8 ${ }^{\circ} \mathrm{C}$, eluent: EA/PE $=1: 10 ;{ }^{1} \mathrm{H}$ NMR (400 $\left.\mathrm{MHz}, \mathrm{CDCl}_{3}\right) \delta 7.83-7.79(\mathrm{~m}, 1 \mathrm{H}), 7.52-7.47(\mathrm{~m}, 4 \mathrm{H}), 7.30-7.27(\mathrm{~m}, 2 \mathrm{H}), 7.07-$ 6.99 (m, 1H), $5.51(\mathrm{~s}, 1 \mathrm{H}), 3.94$ (hept, $J=6.8 \mathrm{~Hz}, 1 \mathrm{H}), 1.46$ (d, $J=6.8 \mathrm{~Hz}, 3 \mathrm{H}), 1.13$ $(\mathrm{d}, J=6.8 \mathrm{~Hz}, 3 \mathrm{H}) ;{ }^{13} \mathrm{C} \mathrm{NMR}\left(100 \mathrm{MHz}, \mathrm{CDCl}_{3}\right) \delta 138.9,137.7,134.2,132.9,132.3$, 129.4, 129.3, 124.8, 122.8, 121.0, 61.6, 46.7, 22.0, 20.3; HRMS (APCI-TOF) m/z: $[\mathrm{M}+\mathrm{H}]^{+}$Calcd for $\mathrm{C}_{16} \mathrm{H}_{17} \mathrm{BrNO}_{2} \mathrm{~S}$ 366.0158; Found 366.0165.

3-(4-chlorophenyl)-2-isopropyl-2,3-dihydrobenzo[d]isothiazole 1,1-dioxide (4k)

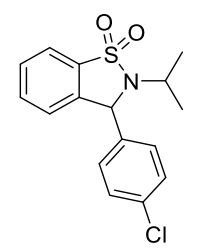

White solid; $53 \mathrm{mg}, 82 \%$ yield; m.p. $91.5-92.6{ }^{\circ} \mathrm{C}$, eluent: $\mathrm{EA} / \mathrm{PE}=1: 10 ;{ }^{1} \mathrm{H} \mathrm{NMR}(400$ $\left.\mathrm{MHz}, \mathrm{CDCl}_{3}\right) \delta 7.83-7.79(\mathrm{~m}, 1 \mathrm{H}), 7.53-7.47(\mathrm{~m}, 2 \mathrm{H}), 7.35(\mathrm{~s}, 4 \mathrm{H}), 7.04-6.98(\mathrm{~m}$, 1H), 5.52 (s, 1H), 3.94 (hept, $J=6.8 \mathrm{~Hz}, 1 \mathrm{H}), 1.46(\mathrm{~d}, J=6.8 \mathrm{~Hz}, 3 \mathrm{H}), 1.13$ (d, $J=6.8$ $\mathrm{Hz}, 3 \mathrm{H}) ;{ }^{13} \mathrm{C} \mathrm{NMR}\left(100 \mathrm{MHz}, \mathrm{CDCl}_{3}\right) \delta 138.3,137.8,134.7,134.2,132.9,129.4,129.3$, 129.0, 124.9, 121.0, 61.6, 46.7, 22.0, 20.3; HRMS (ESI-TOF) m/z: $[\mathrm{M}+\mathrm{Na}]^{+} \mathrm{Calcd}$ for $\mathrm{C}_{16} \mathrm{H}_{16} \mathrm{ClNO}_{2} \mathrm{SNa} 344.0482$; Found 344.0493.

\section{3-(4-fluorophenyl)-2-isopropyl-2,3-dihydrobenzo[d]isothiazole 1,1-dioxide (4l)}

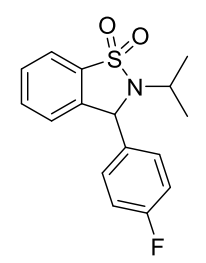

White solid; $49 \mathrm{mg}, 80 \%$ yield; m.p. $118-119^{\circ} \mathrm{C}$, eluent: EA/PE $=1: 10 ;{ }^{1} \mathrm{H}$ NMR $(400$ $\left.\mathrm{MHz}, \mathrm{CDCl}_{3}\right) \delta 7.83-7.78(\mathrm{~m}, 1 \mathrm{H}), 7.54-7.47(\mathrm{~m}, 2 \mathrm{H}), 7.41-7.35(\mathrm{~m}, 2 \mathrm{H}), 7.09-$ $7.04(\mathrm{~m}, 2 \mathrm{H}), 7.03-6.98(\mathrm{~m}, 1 \mathrm{H}), 5.54(\mathrm{~s}, 1 \mathrm{H}), 3.94($ hept, $J=6.8 \mathrm{~Hz}, 1 \mathrm{H}), 1.46(\mathrm{~d}, J$ $=6.8 \mathrm{~Hz}, 3 \mathrm{H}), 1.12(\mathrm{~d}, J=6.8 \mathrm{~Hz}, 3 \mathrm{H}) ;{ }^{13} \mathrm{C} \mathrm{NMR}\left(100 \mathrm{MHz}, \mathrm{CDCl}_{3}\right) \delta 162.9(J=246.5$ $\mathrm{Hz}), 138.1,135.5(J=3.2 \mathrm{~Hz}), 134.2,132.8,129.4,129.3(J=4.9 \mathrm{~Hz}), 124.9,120.9$, 
$116.1(J=21.7 \mathrm{~Hz}), 61.6,46.6,21.9,20.3 ;{ }^{19} \mathrm{~F}$ NMR $\left(375 \mathrm{MHz}, \mathrm{CDCl}_{3}\right) \delta-112.8$; HRMS (ESI-TOF) m/z: $[\mathrm{M}+\mathrm{Na}]^{+} \mathrm{Calcd}$ for $\mathrm{C}_{16} \mathrm{H}_{16} \mathrm{FNO}_{2} \mathrm{SNa}$ 328.0778; Found 328.0786 .

\section{3-(2-bromophenyl)-2-isopropyl-2,3-dihydrobenzo[d]isothiazole 1,1-dioxide (4m)}

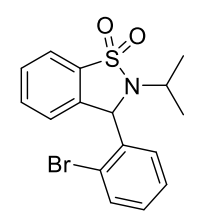

White solid; $56.4 \mathrm{mg}, 77 \%$ yield; m.p. $182.8-183.3{ }^{\circ} \mathrm{C}$, eluent: $\mathrm{EA} / \mathrm{PE}=1: 10 ;{ }^{1} \mathrm{H} \mathrm{NMR}$ $\left(600 \mathrm{MHz}\right.$, Acetone-d $\left.\mathrm{d}_{6}\right) \delta 7.86-7.83(\mathrm{~m}, 1 \mathrm{H}), 7.74-7.73(\mathrm{~m}, 1 \mathrm{H}), 7.67-7.64(\mathrm{~m}, 2 \mathrm{H})$, $7.44-7.38(\mathrm{~m}, 2 \mathrm{H}), 7.33-7.29(\mathrm{~m}, 2 \mathrm{H}), 6.29(\mathrm{~s}, 1 \mathrm{H}), 3.96-3.89(\mathrm{~m}, 1 \mathrm{H}), 1.43(\mathrm{~d}, J$ $=6.6 \mathrm{~Hz}, 3 \mathrm{H}), 1.11(\mathrm{~d}, J=6.6 \mathrm{~Hz}, 3 \mathrm{H}) ;{ }^{13} \mathrm{C} \mathrm{NMR}\left(150 \mathrm{MHz}, \mathrm{CDCl}_{3}\right) \delta 137.6,134.1$, 133.04, 132.96, 130.0, 129.8, 129.4, 128.6, 124.7, 122.5, 121.1, 60.4, 46.9, 22.2, 20.3; HRMS (APCI-TOF) m/z: [M+H] $]^{+}$Calcd for $\mathrm{C}_{16} \mathrm{H}_{17} \mathrm{BrNO}_{2} \mathrm{~S}$ 366.0158; Found 366.0172. 3-(2-bromo-5-fluorophenyl)-2-isopropyl-2,3-dihydrobenzo[d]isothiazole1,1dioxide (4n)

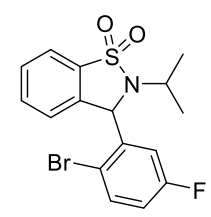

White solid; $46.9 \mathrm{mg}, 61 \%$ yield; m.p. $137.5-138.6{ }^{\circ} \mathrm{C}$, eluent: $\mathrm{EA} / \mathrm{PE}=1: 10 ;{ }^{1} \mathrm{H}$ NMR $(600 \mathrm{MHz}$, Acetone-d 6$) \delta 7.88-7.85(\mathrm{~m}, 1 \mathrm{H}), 7.80(\mathrm{dd}, J=9.0,5.4 \mathrm{~Hz}, 1 \mathrm{H}), 7.70-$ $7.66(\mathrm{~m}, 2 \mathrm{H}), 7.37-7.35(\mathrm{~m}, 1 \mathrm{H}), 7.22(\mathrm{dd}, J=9.0,2.4 \mathrm{~Hz}, 1 \mathrm{H}), 7.17-7.14(\mathrm{~m}, 1 \mathrm{H})$, $6.27(\mathrm{~s}, 1 \mathrm{H}), 4.00-3.94(\mathrm{~m}, 1 \mathrm{H}), 1.43(\mathrm{~d}, J=7.2 \mathrm{~Hz}, 3 \mathrm{H}), 1.15(\mathrm{~d}, J=6.6 \mathrm{~Hz}, 3 \mathrm{H}) ;{ }^{13} \mathrm{C}$ NMR $\left(150 \mathrm{MHz}, \mathrm{CDCl}_{3}\right) \delta 162.5(J=248.0 \mathrm{~Hz}), 142.0,136.9,134.4(J=7.4 \mathrm{~Hz}), 134.1$, 133.1, 129.6, 124.5, 121.2, $117.7(J=22.8 \mathrm{~Hz}), 116.5(\mathrm{~J}=24.6 \mathrm{~Hz}), 116.3(\mathrm{~J}=3.0 \mathrm{~Hz})$, 60.3, 47.1, 22.2, 20.2; ${ }^{19} \mathrm{~F}$ NMR (375 MHz, $\left.\mathrm{CDCl}_{3}\right) \delta-111.5$; HRMS (APCI-TOF) m/z: $[\mathrm{M}+\mathrm{H}]^{+}$Calcd for $\mathrm{C}_{16} \mathrm{H}_{16} \mathrm{BrFNO}_{2} \mathrm{~S}$ 384.0064; Found 384.0081.

\section{3-(3-chlorophenyl)-2-isopropyl-2,3-dihydrobenzo[d]isothiazole 1,1-dioxide (4o)}

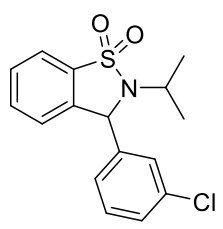


White solid; 45.7mg, 71\% yield; m.p. $114-115{ }^{\circ} \mathrm{C}$, eluent: EA/PE $=1: 10 ;{ }^{1} \mathrm{H}$ NMR (400 $\left.\mathrm{MHz}, \mathrm{CDCl}_{3}\right) \delta 7.84-7.79(\mathrm{~m}, 1 \mathrm{H}), 7.54-7.48(\mathrm{~m}, 2 \mathrm{H}), 7.42-7.39(\mathrm{~m}, 1 \mathrm{H}), 7.32-$ 7.29 (m, 3H), $7.07-7.02(\mathrm{~m}, 1 \mathrm{H}), 5.51$ (s, 1H), 3.95 (hept, $J=6.8 \mathrm{~Hz}, 1 \mathrm{H}), 1.47$ (d, $J$ $=6.8 \mathrm{~Hz}, 3 \mathrm{H}), 1.15(\mathrm{~d}, J=6.8 \mathrm{~Hz}, 3 \mathrm{H}) ;{ }^{13} \mathrm{C} \mathrm{NMR}\left(100 \mathrm{MHz}, \mathrm{CDCl}_{3}\right) \delta 141.9,137.5$, $135.0,134.2,132.9,130.4,129.4,129.0,127.6,125.8,124.9,121.0,61.7,46.8,22.0$, 20.3; HRMS (ESI-TOF) m/z: $[\mathrm{M}+\mathrm{Na}]^{+}$Calcd for $\mathrm{C}_{16} \mathrm{H}_{16} \mathrm{ClNO}_{2} \mathrm{SNa} 344.0482$; Found 344.0491 .

\section{3-(2-isopropyl-1,1-dioxido-2,3-dihydrobenzo[d]isothiazol-3-yl)benzonitrile (4p)}

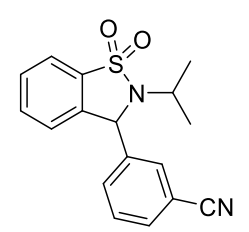

White solid; $38.7 \mathrm{mg}$, $62 \%$ yield; m.p. $44.3-44.9{ }^{\circ} \mathrm{C}$, eluent: EA/PE $=1: 5 ;{ }^{1} \mathrm{H}$ NMR $\left(600 \mathrm{MHz}, \mathrm{CDCl}_{3}\right) \delta 7.84-7.83(\mathrm{~m}, 1 \mathrm{H}), 7.74-7.73(\mathrm{~m}, 1 \mathrm{H}), 7.67(\mathrm{dt}, J=7.8,1.2 \mathrm{~Hz}$, 1H), $7.65(\mathrm{dt}, J=7.8,1.2 \mathrm{~Hz}, 1 \mathrm{H}), 7.56-7.49(\mathrm{~m}, 3 \mathrm{H}), 7.02-7.01 \mathrm{~m}, 1 \mathrm{H}), 5.58(\mathrm{~s}$, $1 \mathrm{H}), 4.00-3.93(\mathrm{~m}, 1 \mathrm{H}), 1.46(\mathrm{~d}, J=6.8 \mathrm{~Hz}, 3 \mathrm{H}), 1.13(\mathrm{~d}, J=6.8 \mathrm{~Hz}, 3 \mathrm{H}) ;{ }^{13} \mathrm{C} \mathrm{NMR}$ $\left(150 \mathrm{MHz}, \mathrm{CDCl}_{3}\right) \delta 141.8,136.9,134.2,133.1,132.5,131.9,130.9,130.1,129.7$, $124.8,121.1,118.1,113.3,61.2,46.9,22.0,20.2$; HRMS (ESI-TOF) m/z: [M+Na] Calcd for $\mathrm{C}_{17} \mathrm{H}_{16} \mathrm{~N}_{2} \mathrm{O}_{2} \mathrm{SNa}^{+}$335.0825; Found 335.0834.

2-isopropyl-5-methyl-3-phenyl-2,3-dihydrobenzo[d]isothiazole 1,1-dioxide (4q)

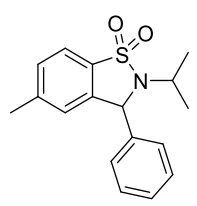

White solid; $45.8 \mathrm{mg}, 76 \%$ yield; m.p. $105-106{ }^{\circ} \mathrm{C}$, eluent: EA/PE $=1: 10 ;{ }^{1} \mathrm{H}$ NMR $\left(400 \mathrm{MHz}, \mathrm{CDCl}_{3}\right) \delta 7.68(\mathrm{~d}, J=8.0 \mathrm{~Hz}, 1 \mathrm{H}), 7.41-7.32(\mathrm{~m}, 5 \mathrm{H}), 7.28(\mathrm{~d}, J=8.0 \mathrm{~Hz}$, 1H), 6.79 (s, 1H), 5.49 (s, 1H), 3.93 (hept, $J=6.8 \mathrm{~Hz}, 1 \mathrm{H}), 2.32$ (s, 3H), 1.46 (d, $J=$ $6.8 \mathrm{~Hz}, 3 \mathrm{H}), 1.11(\mathrm{~d}, J=6.8 \mathrm{~Hz}, 3 \mathrm{H}) ;{ }^{13} \mathrm{C} \mathrm{NMR}\left(100 \mathrm{MHz}, \mathrm{CDCl}_{3}\right) \delta 143.6,139.8$, 138.6, 131.6, 130.2, 129.0, 128.6, 127.7, 125.1, 120.6, 62.2, 46.6, 21.9, 21.8, 20.3; HRMS (ESI-TOF) m/z: $[\mathrm{M}+\mathrm{Na}]^{+}$Calcd for $\mathrm{C}_{17} \mathrm{H}_{19} \mathrm{NO}_{2} \mathrm{SNa}$ 324.1029; Found: 324.1038 . 
2-isopropyl-6-methyl-3-phenyl-2,3-dihydrobenzo[d]isothiazole 1,1-dioxide (4r)

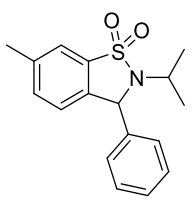

White solid; $49.4 \mathrm{mg}, 82 \%$ yield, eluent: EA/PE $=1: 10 ;{ }^{1} \mathrm{H}$ NMR $(600 \mathrm{MHz}$, Acetoned $\mathrm{d}_{6} \delta 7.58(\mathrm{~s}, 1 \mathrm{H}), 7.51-7.50(\mathrm{~m}, 2 \mathrm{H}), 7.42-7.39(\mathrm{~m}, 3 \mathrm{H}), 7.36-7.34(\mathrm{~m}, 1 \mathrm{H}), 7.03$ $(\mathrm{d}, J=7.8 \mathrm{~Hz}, 1 \mathrm{H}), 5.76(\mathrm{~s}, 1 \mathrm{H}), 3.86($ hept $J=6.6 \mathrm{~Hz} 1 \mathrm{H}), 2.44(\mathrm{~s}, 3 \mathrm{H}), 1.42(\mathrm{~d}, J=$ $6.6 \mathrm{~Hz}, 3 \mathrm{H}), 1.12(\mathrm{~d}, J=6.6 \mathrm{~Hz}, 3 \mathrm{H}) ;{ }^{13} \mathrm{C}$ NMR (150 MHz, Acetone-d 6$) \delta 142.2,141.0$, 137.2, 136.2, 135.0, 130.1, 129.6, 128.8, 126.23, 121.3, 62.9, 47.9, 22.5, 21.4, 20.8; HRMS (ESI-TOF) m/z: [M+Na $]^{+}$Calcd for $\mathrm{C}_{17} \mathrm{H}_{19} \mathrm{NO}_{2} \mathrm{SNa} 324.1029$; Found 324.1038. 5-chloro-2-isopropyl-3-phenyl-2,3-dihydrobenzo[d]isothiazole 1,1-dioxide (4s)

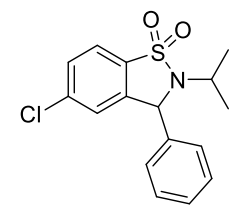

White solid; $52.8 \mathrm{mg}, 82 \%$ yield; m.p. 108.2-109.2 ${ }^{\circ} \mathrm{C}$, eluent: $\mathrm{EA} / \mathrm{PE}=1: 10 ;{ }^{1} \mathrm{H}$ NMR $(600 \mathrm{MHz}$, Acetone-d 6$) \delta 7.85(\mathrm{~d}, J=8.4 \mathrm{~Hz}, 1 \mathrm{H}), 7.64(\mathrm{ddd}, J=8.4,2.1,0.6 \mathrm{~Hz}, 1 \mathrm{H})$, 7.55- $7.54(\mathrm{~m}, 2 \mathrm{H}), 7.45-7.43(\mathrm{~m}, 2 \mathrm{H}), 7.40-7.38(\mathrm{~m}, 1 \mathrm{H}), 7.21-7.20(\mathrm{~m}, 1 \mathrm{H}), 5.86$ (s, 1H), 3.95 (hept, $J=6.6 \mathrm{~Hz}, 1 \mathrm{H}), 1.43(\mathrm{~d}, J=6.6 \mathrm{~Hz}, 3 \mathrm{H}), 1.13(\mathrm{~d}, J=6.6 \mathrm{~Hz}, 3 \mathrm{H})$; ${ }^{13} \mathrm{C} \mathrm{NMR}\left(150 \mathrm{MHz}, \mathrm{CDCl}_{3}\right) \delta 140.4,139.2,138.8,132.8,129.8,129.3,129.1,127.6$, 125.2, 122.2, 62.0, 46.8, 21.8, 20.3; HRMS (APCI-TOF) m/z: $[\mathrm{M}+\mathrm{H}]^{+}$Calcd for $\mathrm{C}_{16} \mathrm{H}_{17} \mathrm{ClNO}_{2} \mathrm{~S}$ 322.0663; Found 322.0664.

\section{4-chloro-2-isopropyl-3-phenyl-2,3-dihydrobenzo[d]isothiazole 1,1-dioxide (4t)}

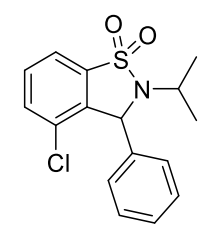

White solid; $54 \mathrm{mg}, 84 \%$ yield; m.p. $137.0-137.7^{\circ} \mathrm{C}$, eluent: EA/PE $=1: 10 ;{ }^{1} \mathrm{H}$ NMR $\left(600 \mathrm{MHz}, \mathrm{CDCl}_{3}\right) \delta 7.78(\mathrm{dd}, J=7.8,1.2 \mathrm{~Hz}, 1 \mathrm{H}), 7.52-7.47(\mathrm{~m}, 2 \mathrm{H}), 7.42(\mathrm{dd}, J=$ 7.5, $2.0 \mathrm{~Hz}, 2 \mathrm{H}), 7.38-7.29$ (m, 3H), 5.57 (s, 1H), $3.92-3.85$ (hept, $J=7.2 \mathrm{~Hz}, 1 \mathrm{H}$ ), $1.46(\mathrm{~d}, J=7.2 \mathrm{~Hz}, 3 \mathrm{H}), 1.09(\mathrm{~d}, J=7.2 \mathrm{~Hz}, 3 \mathrm{H}) ;{ }^{13} \mathrm{C} \mathrm{NMR}\left(100 \mathrm{MHz}, \mathrm{CDCl}_{3}\right) \delta$ 137.24, 137.20, 135.4, 133.5, 131.2, 131.0, 129.05, 128.7, 128.6, 119.4, 62.0, 46.5, 21.9, 
20.3; HRMS (APCI-TOF) m/z: [M+H] $]^{+}$Calcd for $\mathrm{C}_{16} \mathrm{H}_{17} \mathrm{ClNO}_{2} \mathrm{~S}$ 322.0663; Found 322.0667 .

\section{7-chloro-2-isopropyl-3-phenyl-2,3-dihydrobenzo[d]isothiazole 1,1-dioxide (4u)}

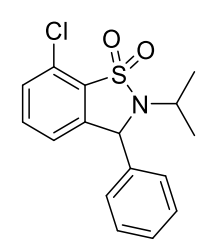

White solid; $22 \mathrm{mg}, 34 \%$ yield, eluent: EA/PE $=1: 10 ;{ }^{1} \mathrm{H}$ NMR $\left(600 \mathrm{MHz}, \mathrm{DMSO}-\mathrm{d}_{6}\right)$ $\delta 7.66-7.61(\mathrm{~m}, 2 \mathrm{H}), 7.47(\mathrm{~d}, J=7.8 \mathrm{~Hz}, 2 \mathrm{H}), 7.41(\mathrm{t}, J=7.2 \mathrm{~Hz}, 2 \mathrm{H}), 7.34(\mathrm{t}, J=7.2$ $\mathrm{Hz}, 1 \mathrm{H}), 7.14(\mathrm{~d}, J=7.2 \mathrm{~Hz}, 1 \mathrm{H}), 5.94(\mathrm{~s}, 1 \mathrm{H}), 3.83$ (hept, $J=6.6 \mathrm{~Hz}, 1 \mathrm{H}), 1.35$ (d, $J$ $=6.6 \mathrm{~Hz}, 3 \mathrm{H}), 1.03(\mathrm{~d}, J=6.6 \mathrm{~Hz}, 3 \mathrm{H}) ;{ }^{13} \mathrm{C} \mathrm{NMR}\left(150 \mathrm{MHz}, \mathrm{DMSO}-\mathrm{d}_{6}\right) \delta 141.3,140.0$, $134.9,131.1,130.0,129.0,128.5,127.2$, 126.2, 124.3, 60.2, 46.6, 21.7, 19.9; HRMS (APCI-TOF) m/z: [M+H] $]^{+}$Calcd for $\mathrm{C}_{16} \mathrm{H}_{17} \mathrm{ClNO}_{2} \mathrm{~S}$ 322.0663; Found: 322.0669 .

\subsection{Large-scale synthesis of 4a:}

Substrate 3a (1.0 mmol, $0.29 \mathrm{~g}), n$-Bu 4 NOAc (1.0 mmol, $0.3 \mathrm{~g})$ and a mixture of MeCN and HFIP (V/V $20 \mathrm{~mL} / 10 \mathrm{~mL}$ ) were successively added to a $100 \mathrm{~mL}$ beaker equipped with carbon rod ( $\phi 6 \mathrm{~mm}$, about $10 \mathrm{~mm}$ immersion depth in solution) as the anode and platinum plate $(1 \mathrm{~cm} \times 1 \mathrm{c} \mathrm{mm})$ as the cathode as a undivided beaker-type cell. Under argon atmosphere, the reaction mixture was stirred and electrolyzed at a constant current of $15 \mathrm{~mA}$ for $8 \mathrm{~h}$ at $\mathrm{rt}$. Upon completion, the reaction mixture was transferred to a single-necked flask and the reaction mixture was concentrated under reduced pressure. The residue was chromatographed by silica gel chromatography $\left(\mathrm{V}_{\text {EtOAd }} / \mathrm{V}_{\text {petroleum ether }} 1: 10\right)$ to give the desired product $4 \mathbf{a}(0.19 \mathrm{~g}, 66 \%)$. 


\section{Mechanistic studies}

\section{1) Cyclic voltametric studies}

To elucidate the reaction mechanism, cyclic voltametric studies were conducted by using 3a (Figure S2, red line), model compounds 5 (Figure S2, pink line) and 6 (Figure S2, blue line) in $0.1 \mathrm{M} \mathrm{MeCN} / \mathrm{HFIP}$. Each solution contained a centration of $3 \mathrm{mM}$ of the corresponding substrate. By comparing the oxidation potentials of the three model substrates, 3a, $\mathbf{5}$ and $\mathbf{6}$, it should be possible to determine whether the $\mathrm{N}$ atom in sulfonamide or the aromatic group is oxidized first. Initially, the CV of the blank electrolyte $0.1 \mathrm{M}^{n} \mathrm{Bu}_{4} \mathrm{NClO}_{4}$ in MeCN/HFIP (black line) was measured, demonstrating the stability of the current electrolytes towards anodic oxidation. Substrate 3a, bearing both the sulfamido and benzyl group, is irreversibly oxidized at a potential of $1.97 \mathrm{~V}$. The oxidation for compound $\mathbf{5}$ without the sulfamido group takes place at $1.90 \mathrm{~V}$, close to that of $\mathbf{3 a}$, whereas benzenesulfonamide $\mathbf{6}$ without a benzyl group was not oxidized at all in the range of between 0 and $2.5 \mathrm{~V}$. The results depicted in Figure S2 gave strong support for the mechanism through aromatic radical cations.

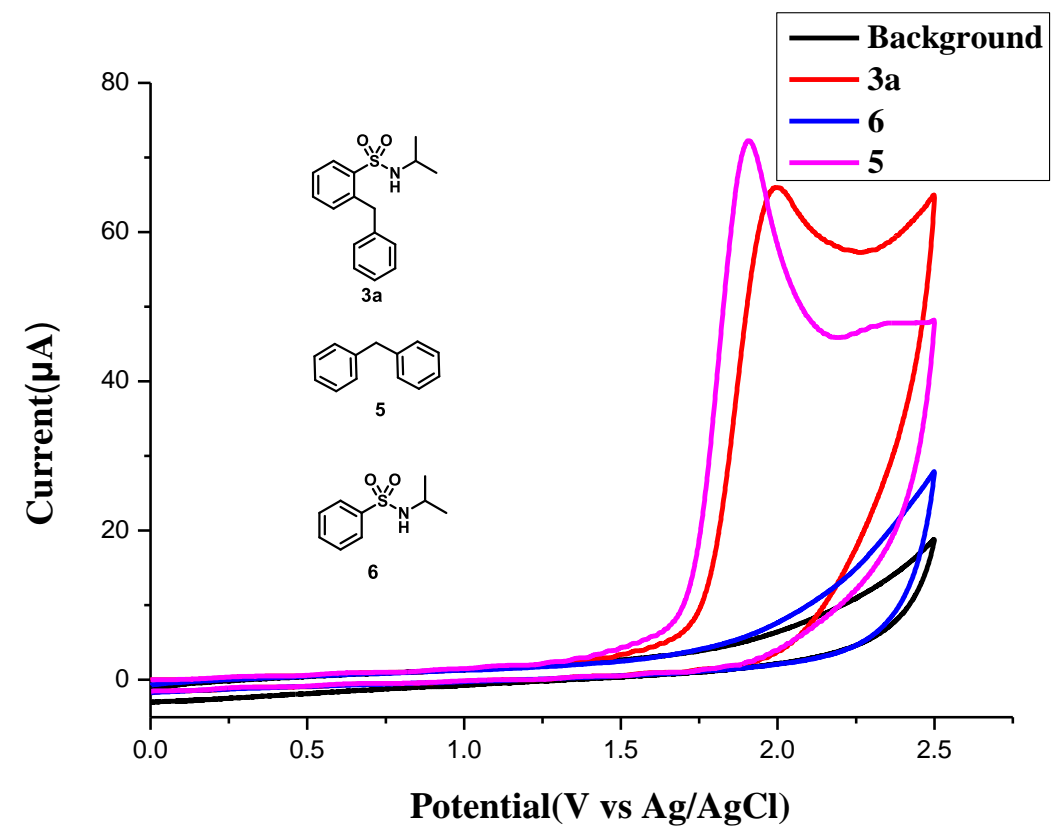

Figure S2 Cyclic voltammetry of 3a (3 mM, red line), 5 (3 mM, pink line) and $\mathbf{6}$ (3 $\mathrm{mM}$, blue line). The voltammogram for the blank electrolyte is shown for comparison (black line). Working electrode: glassy carbon; counter electrode: $\mathrm{Pt}(5 \mathrm{~mm}$ x $5 \mathrm{~mm} \mathrm{x}$ $0.1 \mathrm{~mm}$ ); reference electrode: $\mathrm{Ag} / \mathrm{AgCl}, 0.1 \mathrm{M} \mathrm{Bu}_{4} \mathrm{NClO}_{4}$ in $\mathrm{MeCN} / \mathrm{HFIP}(2: 1=3 \mathrm{~mL})$, scan rate: $50 \mathrm{mVs}^{-1}$. 


\section{2) Control experiment}

A control experiment was also performed. It was found that when using an ortho-methyl substituted benzenesulfonamide $7^{16}$ instead of a benzyl substituted one as substrate, the reaction became relatively sluggish under the optimal for the five-membered ring formation. Apart from the unreacted substrate 7, complicated product mixtures were also observed. However, after analyzed by HRMS, the desired five-membered sultam was not detected, which indicates that the mechanism through nitrogen centered radicals ${ }^{17}$ is not the predominant pathway for the electrochemical cyclization.
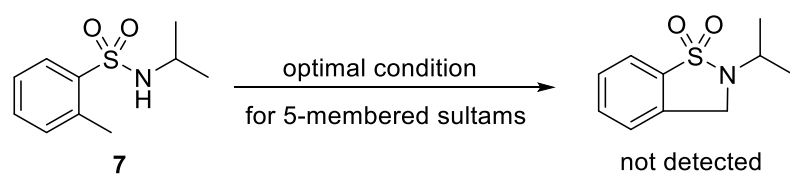

Scheme S2. control experiment

\section{3) Mechanism for seven-membered benzosultams}

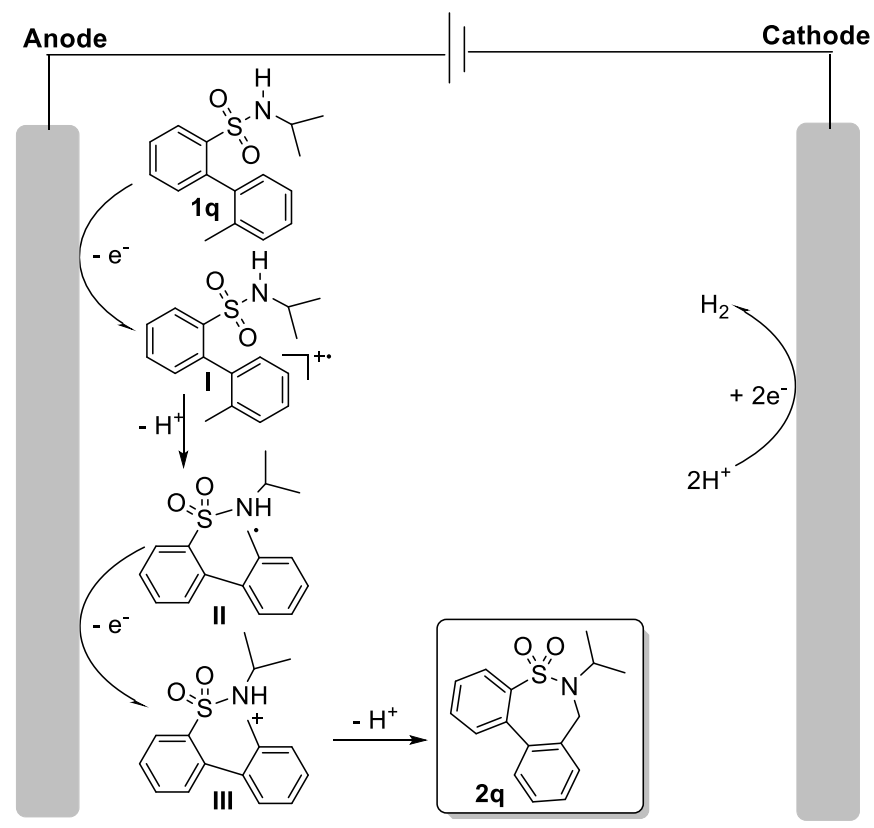

Scheme S3. Proposed mechanism for 7-membered benzosultams

Based on the results of our work and those of previously reported ${ }^{18-20}$, a possible mechanism for the formation of 7-membered benzosultams was proposed in scheme S3: at the outset of the reaction, an aromatic radical cation $\mathbf{I}$ is generated via the anodic oxidation. I then loses a benzylic proton to furnish radical II, which undergoes the 
second single-electron transfer (SET) oxidation in the anode, delivering the benzylic cation III. The intramolecular nucleophilic attack on intermediate III, followed by the proton elimination, results in the target 7-membered cyclic product $\mathbf{2 q}$. 


\section{X-Ray Crystal Structure and Data of 2a, 2q and 2r}

Compound 2a (CCDC:2035936)
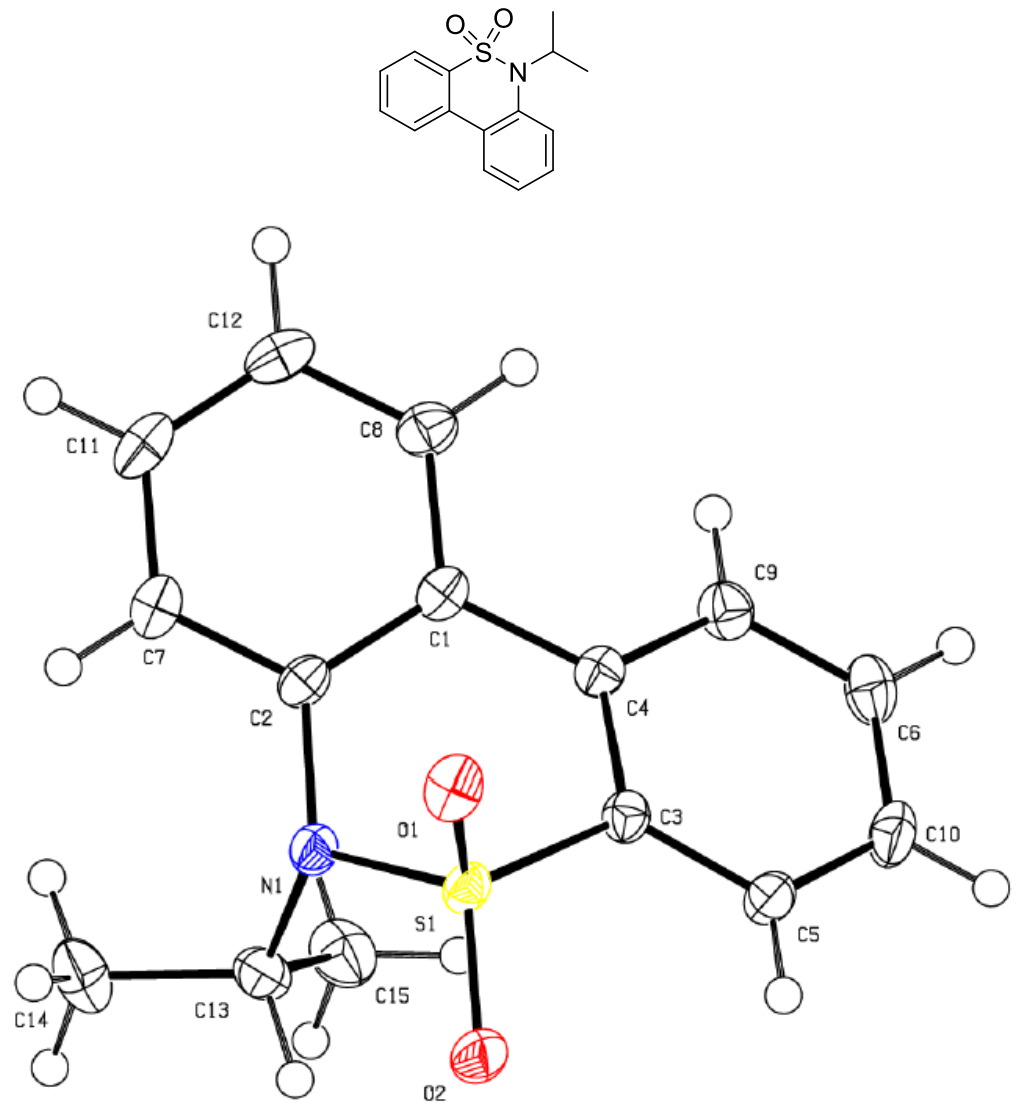

Figure S3. X-ray structure of 2 a with $30 \%$ ellipsoid probability

Table S1. Crystal Data and Structure Refinement for 2a

$\begin{array}{ll}\text { Empirical formula } & \mathrm{C}_{15} \mathrm{H}_{15} \mathrm{NO}_{2} \mathrm{~S} \\ \text { Formula weight } & 273.34 \\ \text { Temperature/K } & 161.0 \\ \text { Crystal system } & \text { monoclinic } \\ \text { Space group } & \mathrm{P} 2{ }_{1} / \mathrm{c} \\ \mathrm{a} / \AA & 9.0768(7) \\ \mathrm{b} / \AA & 7.3377(6) \\ \mathrm{c} / \AA & 19.7209(16) \\ \alpha /{ }^{\circ} & 90 \\ \beta /{ }^{\circ} & 93.310(2) \\ \gamma /{ }^{\circ} & 90 \\ \mathrm{Volume} / \AA^{3} & 1311.28(18) \\ \mathrm{Z} & 4 \\ \rho_{\text {calc }} / \mathrm{cm}^{3} & 1.385 \\ \mu / \mathrm{mm}^{-1} & 2.169 \\ \mathrm{~F}(000) & 576.0\end{array}$


Crystal size $/ \mathrm{mm}^{3} \quad 0.5 \times 0.4 \times 0.3$

Radiation $\quad \mathrm{CuK} \alpha(\lambda=1.54178)$

$2 \Theta$ range for data collection $/{ }^{\circ} 8.982$ to 136.414

Index ranges $\quad-10 \leq \mathrm{h} \leq 10,-8 \leq \mathrm{k} \leq 8,-23 \leq 1 \leq 23$

Reflections collected $\quad 17828$

Independent reflections $\quad 2382\left[\mathrm{R}_{\text {int }}=0.0283, \mathrm{R}_{\text {sigma }}=0.0174\right]$

Data/restraints/parameters $\quad 2382 / 0 / 174$

Goodness-of-fit on $\mathrm{F}^{2} \quad 1.094$

Final $R$ indexes $[\mathrm{I}>=2 \sigma(\mathrm{I})] \quad \mathrm{R}_{1}=0.0302, \mathrm{wR}_{2}=0.0773$

Final $\mathrm{R}$ indexes [all data] $\quad \mathrm{R}_{1}=0.0303, \mathrm{wR}_{2}=0.0774$

Largest diff. peak/hole / e $\AA^{-3} 0.28 /-0.35$

\section{Preparation of the crystal}

The $5 \mathrm{mg}$ of pure compound 2a was dissolved in $0.5 \mathrm{~mL}$ EA in $1.5 \mathrm{~mL}$ bottle. And 5 $\mathrm{mL}$ PE was added dropwise in $20 \mathrm{~mL}$ bottle. The little sample bottle was placed into $20 \mathrm{~mL}$ bottle. Then the closed $20 \mathrm{~mL}$ bottle was placed in a quite environment until the well-shaped single crystals formed.

\section{Compound 2q (CCDC:2035935)}

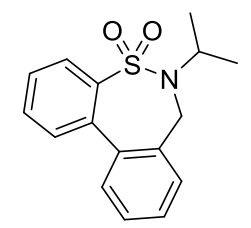




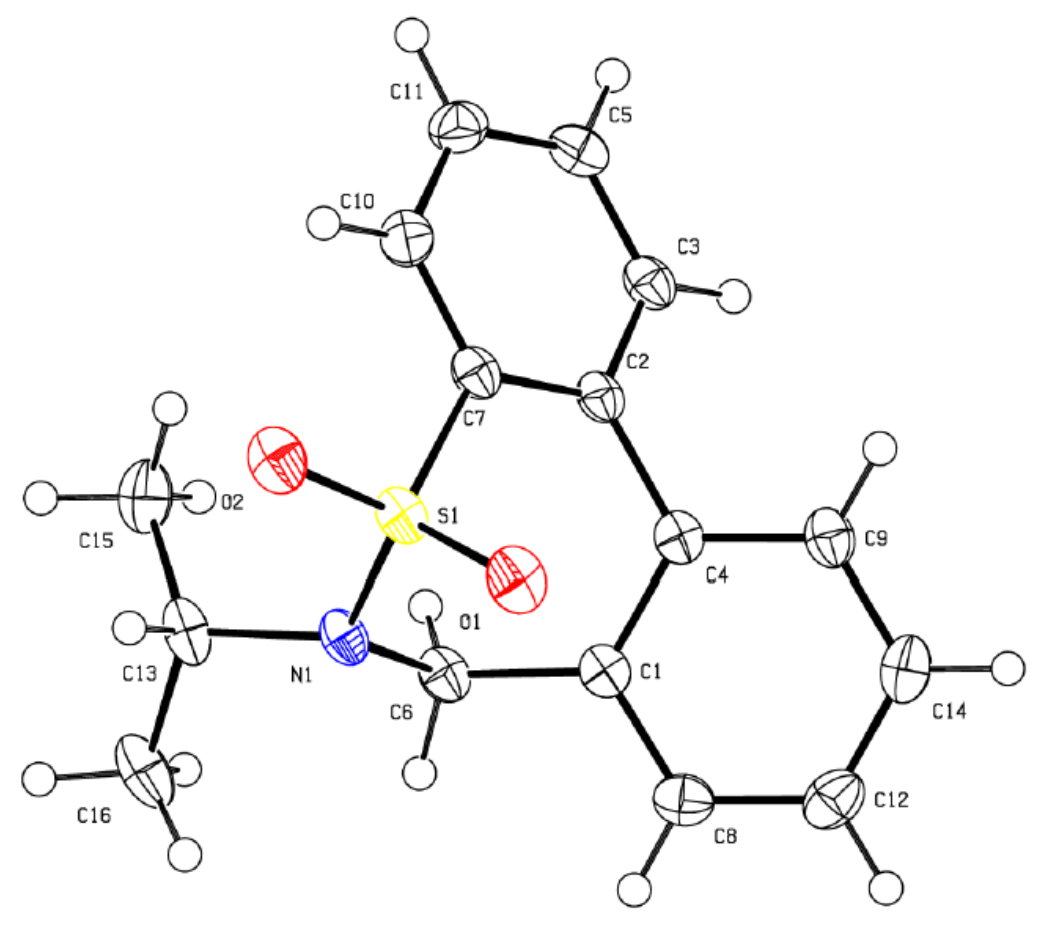

Figure S4. X-ray structure of $2 q$ with $30 \%$ ellipsoid probability

Table S2. Crystal Data and Structure Refinement for $\mathbf{2 q}$

$\begin{array}{ll}\text { Empirical formula } & \mathrm{C}_{16} \mathrm{H}_{17} \mathrm{NO}_{2} \mathrm{~S} \\ \text { Formula weight } & 287.36 \\ \text { Temperature/K } & 153.0 \\ \text { Crystal system } & \text { monoclinic } \\ \text { Space group } & \mathrm{P} 2{ }_{1} / \mathrm{c} \\ \mathrm{a} / \AA & 10.2116(2) \\ \mathrm{b} / \AA & 8.1821(2) \\ \mathrm{c} / \AA & 16.7029(3) \\ \alpha /{ }^{\circ} & 90 \\ \beta /{ }^{\circ} & 96.1780(10) \\ \gamma /{ }^{\circ} & 90 \\ \mathrm{Volume}^{\circ} \AA^{3} & 1387.46(5) \\ \mathrm{Z} & 4 \\ \rho_{\text {calcg }} / \mathrm{cm}^{3} & 1.376 \\ \mu / \mathrm{mm}^{-1} & 2.076 \\ \mathrm{~F}(000) & 608.0 \\ \text { Crystal size } / \mathrm{mm}^{3} & 0.5 \times 0.4 \times 0.3 \\ \text { Radiation } & \mathrm{CuK} \alpha(\lambda=1.54178)\end{array}$

$2 \Theta$ range for data collection/ ${ }^{\circ} 8.71$ to 136.302

Index ranges $\quad-12 \leq \mathrm{h} \leq 12,-9 \leq \mathrm{k} \leq 9,-20 \leq 1 \leq 19$ 
Reflections collected

18752

Independent reflections

Data/restraints/parameters

Goodness-of-fit on $\mathrm{F}^{2}$

Final $\mathrm{R}$ indexes $[\mathrm{I}>=2 \sigma(\mathrm{I})]$

Final $\mathrm{R}$ indexes [all data]

Largest diff. peak/hole / e $\AA^{-3} 0.22 /-0.39$

$2536 / 0 / 183$

1.088

$2536\left[\mathrm{R}_{\text {int }}=0.0383, \mathrm{R}_{\text {sigma }}=0.0219\right]$

$\mathrm{R}_{1}=0.0312, \mathrm{wR}_{2}=0.0780$

$\mathrm{R}_{1}=0.0336, \mathrm{wR}_{2}=0.0798$

\section{Preparation of the crystal}

The $5 \mathrm{mg}$ of pure compound $\mathbf{2 q}$ was dissolved in $0.5 \mathrm{~mL}$ EA in $1.5 \mathrm{~mL}$ bottle. And 5 mL PE was added dropwise in $20 \mathrm{~mL}$ bottle. The little sample bottle was placed into $20 \mathrm{~mL}$ bottle. Then the closed $20 \mathrm{~mL}$ bottle was placed in a quite environment until the well-shaped single crystals formed.

\section{Compound 2r (CCDC:2035937)}
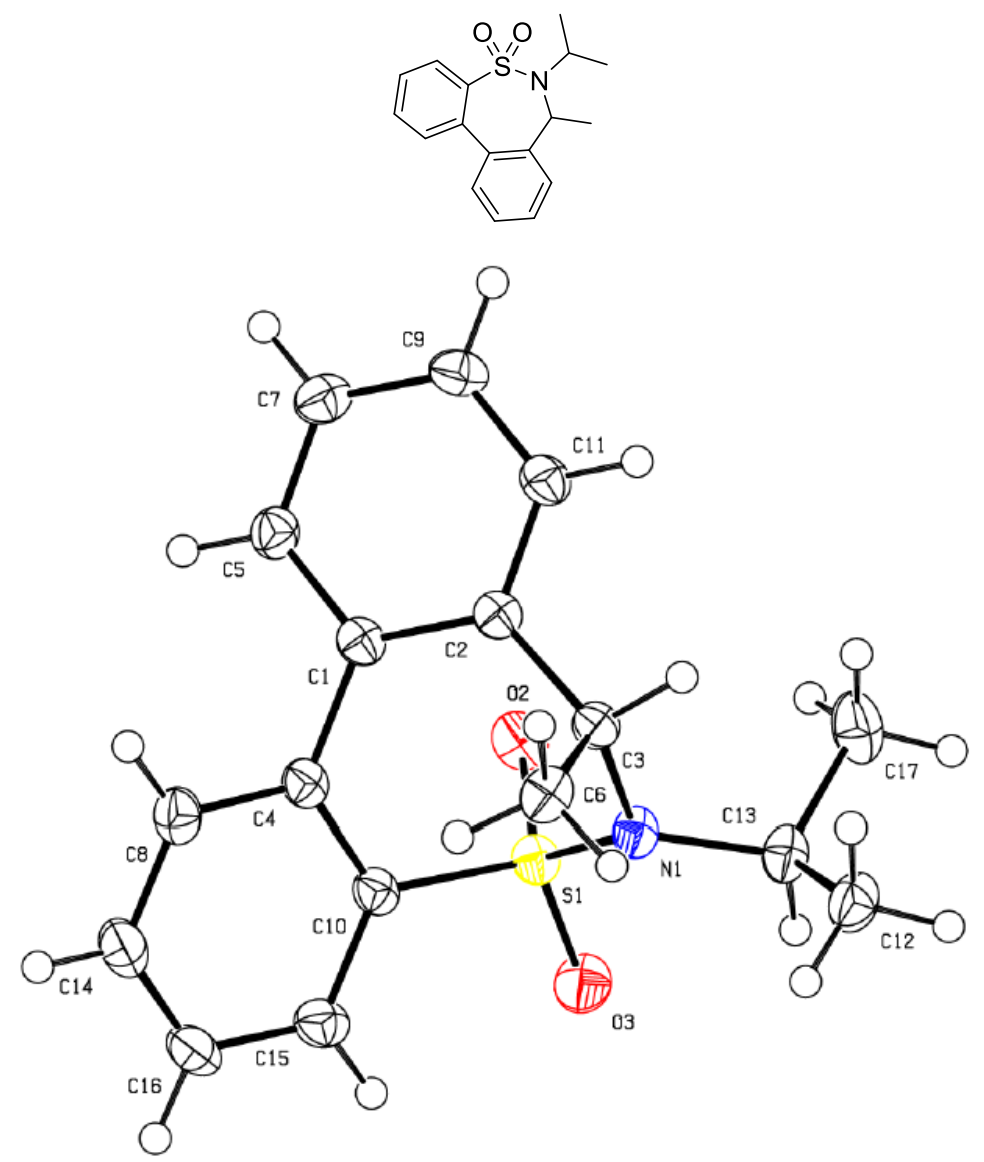

Figure S5. X-ray structure of $2 q$ with $30 \%$ ellipsoid probability

Table S3. Crystal Data and Structure Refinement for $2 \mathbf{r}$ 


\begin{tabular}{|c|c|}
\hline Empirical formula & $\mathrm{C}_{17} \mathrm{H}_{19} \mathrm{NO}_{2} \mathrm{~S}$ \\
\hline Formula weight & 301.39 \\
\hline Temperature/K & 227.0 \\
\hline Crystal system & orthorhombic \\
\hline Space group & $\mathrm{P} 2{ }_{1}{ }_{1} 2_{1}$ \\
\hline $\mathrm{a} / \AA$ & $7.5516(6)$ \\
\hline $\mathrm{b} / \AA$ & $10.6961(8)$ \\
\hline$c / \AA$ & $18.4676(14)$ \\
\hline$\alpha /^{\circ}$ & 90 \\
\hline$\beta /{ }^{\circ}$ & 90 \\
\hline$\gamma /{ }^{\circ}$ & 90 \\
\hline Volume $/ \AA^{3}$ & $1491.7(2)$ \\
\hline $\mathrm{Z}$ & 4 \\
\hline$\rho_{\text {calc }} \mathrm{g} / \mathrm{cm}^{3}$ & 1.342 \\
\hline$\mu / \mathrm{mm}^{-1}$ & 1.955 \\
\hline $\mathrm{F}(000)$ & 640.0 \\
\hline Crystal size $/ \mathrm{mm}^{3}$ & $0.5 \times 0.4 \times 0.4$ \\
\hline Radiation & $\mathrm{CuK} \alpha(\lambda=1.54178)$ \\
\hline \multicolumn{2}{|c|}{$2 \Theta$ range for data collection $/{ }^{\circ} 9.556$ to 137.292} \\
\hline Index ranges & $-9 \leq \mathrm{h} \leq 9,-12 \leq \mathrm{k} \leq 12,-22 \leq 1 \leq 22$ \\
\hline Reflections collected & 19588 \\
\hline Independent reflections & $2719\left[R_{\text {int }}=0.0426, R_{\text {sigma }}=0.0270\right]$ \\
\hline Data/restraints/parameters & $2719 / 0 / 193$ \\
\hline Goodness-of-fit on $\mathrm{F}^{2}$ & 1.082 \\
\hline Final $R$ indexes $[I>=2 \sigma(I)]$ & $\mathrm{R}_{1}=0.0258, \mathrm{wR}_{2}=0.0641$ \\
\hline Final $\mathrm{R}$ indexes [all data] & $\mathrm{R}_{1}=0.0259, \mathrm{wR}_{2}=0.0641$ \\
\hline \multicolumn{2}{|c|}{ Largest diff. peak/hole / e $\AA^{-3} 0.15 /-0.31$} \\
\hline Flack parameter & $0.107(5)$ \\
\hline
\end{tabular}

\section{Preparation of the crystal}

The $5 \mathrm{mg}$ of pure compound $2 \mathbf{r}$ was dissolved in $0.5 \mathrm{~mL}$ EA in $1.5 \mathrm{~mL}$ bottle. And 5 $\mathrm{mL}$ PE was added dropwise in $20 \mathrm{~mL}$ bottle. The little sample bottle was placed into $20 \mathrm{~mL}$ bottle. Then the closed $20 \mathrm{~mL}$ bottle was placed in a quite environment until the well-shaped single crystals formed. 


\section{References}

1. Wallinder, C.; Botros, M.; Rosenstroem, U.; Guimond, M.-O.; Beaudry, H.; Nyberg, F.; Gallo-Payet, N.; Hallberg, A.; Alterman, M. Selective angiotensin II AT2 receptor agonists: Benzamide structure-activity relationships.Bioorg. Med. Chem. 2008, 16, 6841-6849.

2. Rayabarapu, D. K.; Zhou, A.; Jeon, K. O.; Samarakoon, T.; Rolfe, A.; Siddiqui, H.; Hanson, P. R. $\alpha$-Haloarylsulfonamides: multiple cyclization pathways to skeletally diverse benzofused sultams. Tetrahedron, 2009, 65, 3180-3188.

3. Burlein, C.; Wang, C.; Xu, M.; Bhatt, T.; Stahlhut, M.; Ou, Y.; Adam, G. C.; Heath, J.; Klein, D. J.; Sanders, J.; Narayan, K.; Abeywickrema, P.; Heo, M. R.; Carroll, S. S.; Grobler, J. A.; Sharma, S.; Diamond, T. L.; Converso, A.; Krosky, D. J. et al. Discovery of a Distinct Chemical and Mechanistic Class of Allosteric HIV-1 Integrase Inhibitors with Antiretroviral Activity. ACS Chem. Biol. 2017, 12, 2858-2865.

4. Wang, J.; Li, F.; Pei, We.; Yang, M.; Wu, Y.; Ma, D.; Zhang, F.; Wang, J. Selective cleavage of the $\mathrm{N}$-propargyl group from sulfonamides and amides under ruthenium catalysis. Tetrahedron Lett. 2018, 59, 1902-1905.

5. Meyer, D.; Jangra, H.; Walther, F.; Zipse, H.; Renaud, P. A third generation of radical fluorinating agents based on $\mathrm{N}$-fluoro-N-arylsulfonamides. Nat. Commun. 2018, 9, 110.

6. Clavier, H.; Lepronier, A.; Bengobesse-Mintsa, N.; Gatineau, D.; Pellissier, H.; Giordano, L.; Tenaglia, A.; Buono, G. Palladium-Mediated [2+1] Cycloaddition of Norbornene Derivatives with Ynamides. Adv. Synth. Catal. 2013, 355, 403-408.

7. Niu, B.; Xie, P.; Bian, Z.; Zhao, W.; Zhang, M.; Zhou, Y.; Feng, L.; Pittman, C. U.; Zhou, A. Synthesis of Nitromethyl-Substituted Oxindole Derivatives via a Desulfonylation Cascade. Synlett, 2015, 26, 635-638.

8. Martinez, C.; Bosnidou, A. E.; Allmendinger, S.; Muñiz, K. Towards Uniform Iodine Catalysis: Intramolecular C-H Amination of Arenes under Visible Light.Chem. Eur. J. 2016, 22, 9929-9932.

9. Conde, N.; Churruca, F.; San Martin, R.; Herrero, M. T.; Dominguez, E.A further 
decrease in the catalyst loading for the palladium-catalyzed direct intramolecular arylation of amides and sulfonamides. Adv. Synth. Catal. 2015,357, 1525-1531.

10. Bheeter, C. B.; Bera, J. K.; Doucet, H. Palladium-catalyzed intramolecular direct arylation of 2-bromobenzenesulfonic acid derivatives. Adv. Synth. Catal. 2012, 354, 3533-3538.

11. Fruit, C.; Müller, P. Intramolecular asymmetric amidations of sulfonamides and sulfamates catalyzed by chiral dirhodium(II) complexes. Helv. Chim. Acta, 2004, 87, 1607-1615.

12.Lipshutz, B.H.; Ghorai, S.; Leong, W. W. Y. Deprotection of Homoallyl (hAllyl) Derivatives of Phenols, Alcohols, Acids, and Amines. J. Org. Chem. 2009, 74, 7, 2854 2857.

13. Rayabarapu, D. K.; Zhou, A.; Jeon, K. O.; Samarakoon, T.; Rolfe, A.; Siddiqui, H.; Hanson, P. R. $\alpha$-Haloarylsulfonamides: multiple cyclization pathways to skeletally diverse benzofused sultams.Tetrahedron, 2009,65, 3180-3188.

14. Duhamel, T.; Stein, C. J.; Martinez, C.; Reiher, M.; Muñiz, K. Engineering Molecular Iodine Catalysis for Alkyl-Nitrogen Bond Formation. ACS Catal. 2018, 8, $3918-3925$.

15. Bafaluy, D.; Munoz-Molina, J. M.; Funes-Ardoiz, I.; Herold, S.; de Aguirre, A. J.; Zhang, H.; Maseras, F.; Belderrain, T. R.; Perez, P. J.; Muñiz, K.Copper-Catalyzed NF Bond Activation for Uniform Intramolecular C-H Amination Yielding Pyrrolidines and Piperidines. Angew. Chem. Int. Ed. 2019, 58, 8912-8916.

16. Shi, W.; Bai, C.-M.; Zhu, K.; Cui, D.-M.; Zhang, C. Brønsted acid-assisted Nalkylation of sulfonamides using ethers as the alkylation reagents. Tetrahedron $\mathbf{2 0 1 4}$, $70,434-438$.

17. Huang, C.; Li, Z.-Y.; Song, J.; Xu, H.-C. Catalyst- and reagent-free formal azawacker cyclizations enabled by continuous-flow electrochemistry. Angew. Chem. Int. Ed. 2021, 60, 11237-11241.

18. Herold, S.; Bafaluy, D.; Muñiz, K. Anodic benzylic C (sp3)-H amination: unified access to pyrrolidines and piperi-dines. Green Chem. 2018, 20, 3191-3196. 
19. Sutterer, A.; Moeller, K. D. Reversing the polarity of enol ethers: an anodic route to tetrahydrofuran and tetrahydro-pyran rings. J. Am. Chem. Soc. 2000, 122, 56365637.

20. Hou, Z.-W.; Liu, D.-J.; Xiong, P.; Lai, X.-L.; Song, J.; Xu, H.-C. Site-selective electrochemical benzylic C-H amination. Angew. Chem. Int. Ed. 2021, 60, 29432947. 
6 NMR Spectra

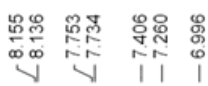

7
0
0
0
1

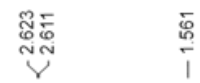

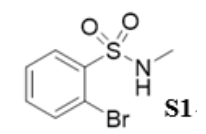

${ }^{1} \mathrm{H}$ NMR spectrum

$400 \mathrm{MHz}, \mathrm{CDCl}_{3}$

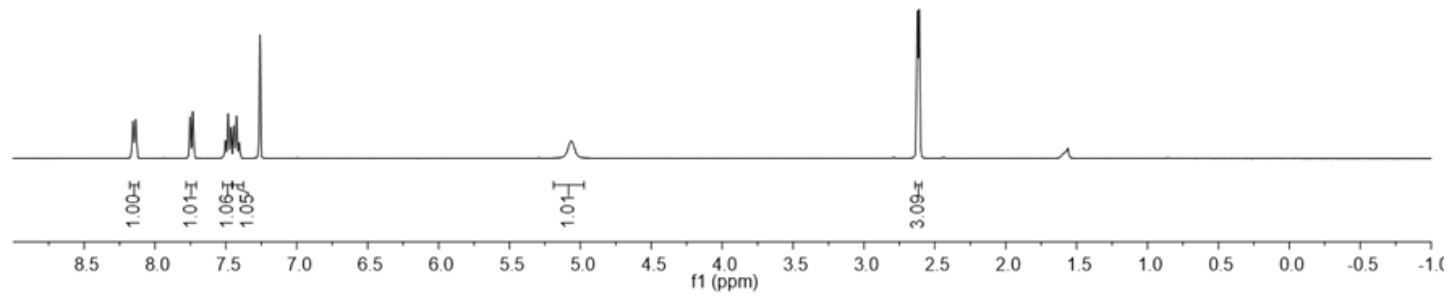

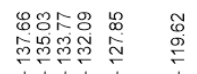

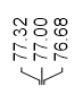

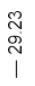

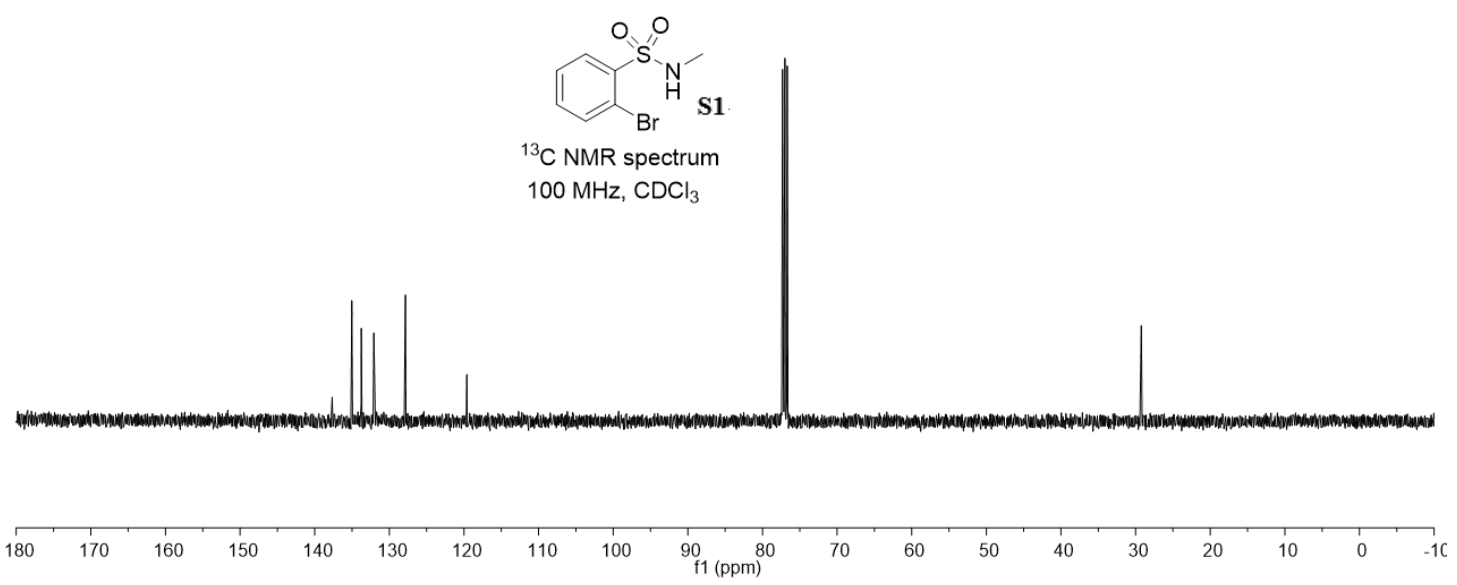



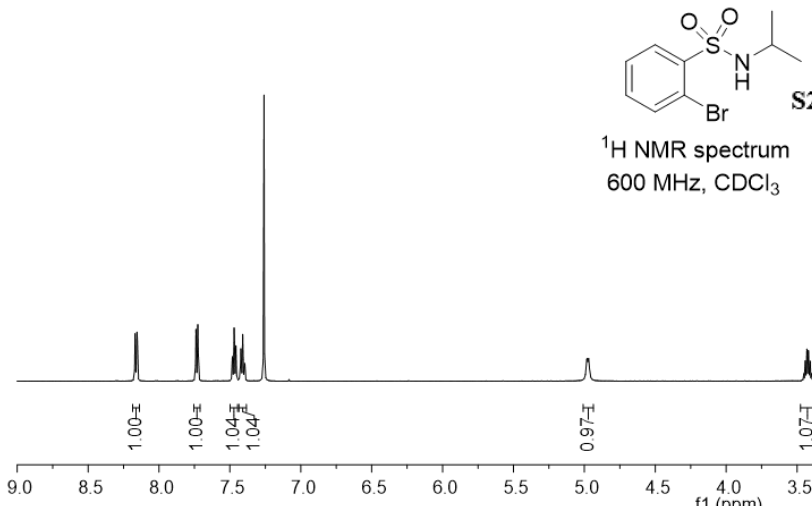

${ }^{1} \mathrm{H}$ NMR spectrum $600 \mathrm{MHz} \mathrm{CDCl}_{3}$

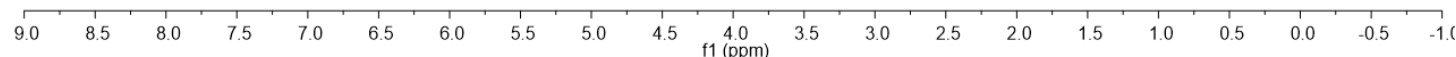
广广

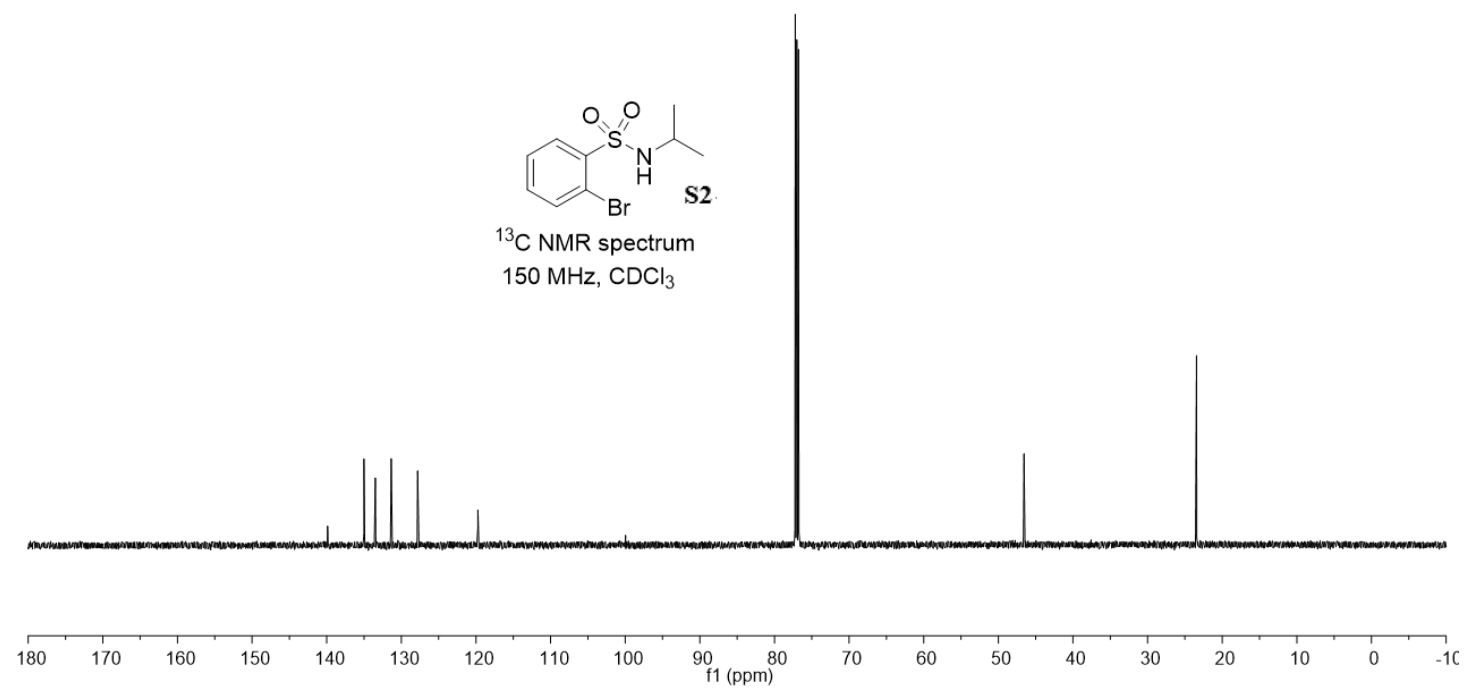




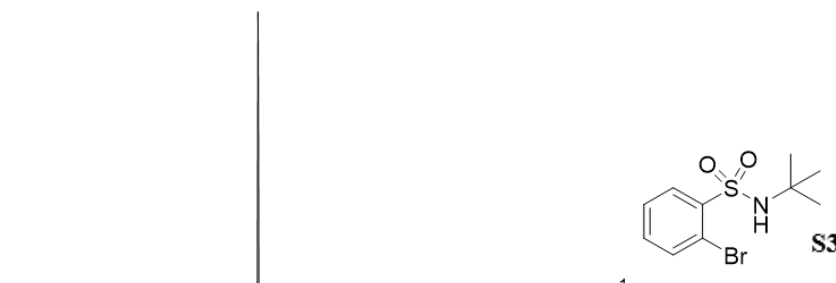

${ }^{1} \mathrm{H}$ NMR spectrum $400 \mathrm{MHz}, \mathrm{CDCl}_{3}$

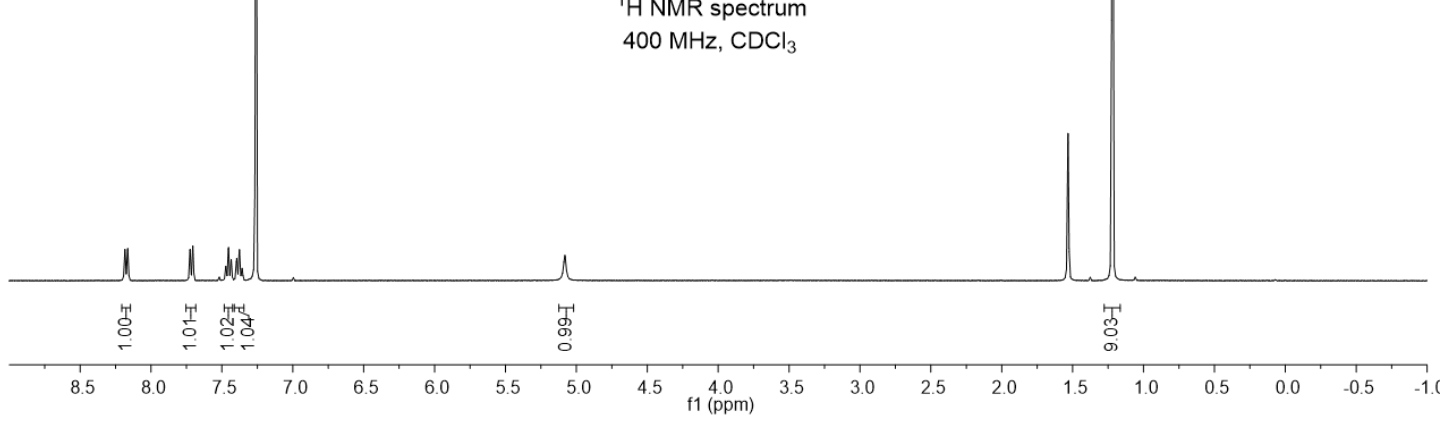
i

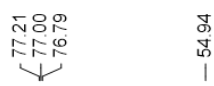

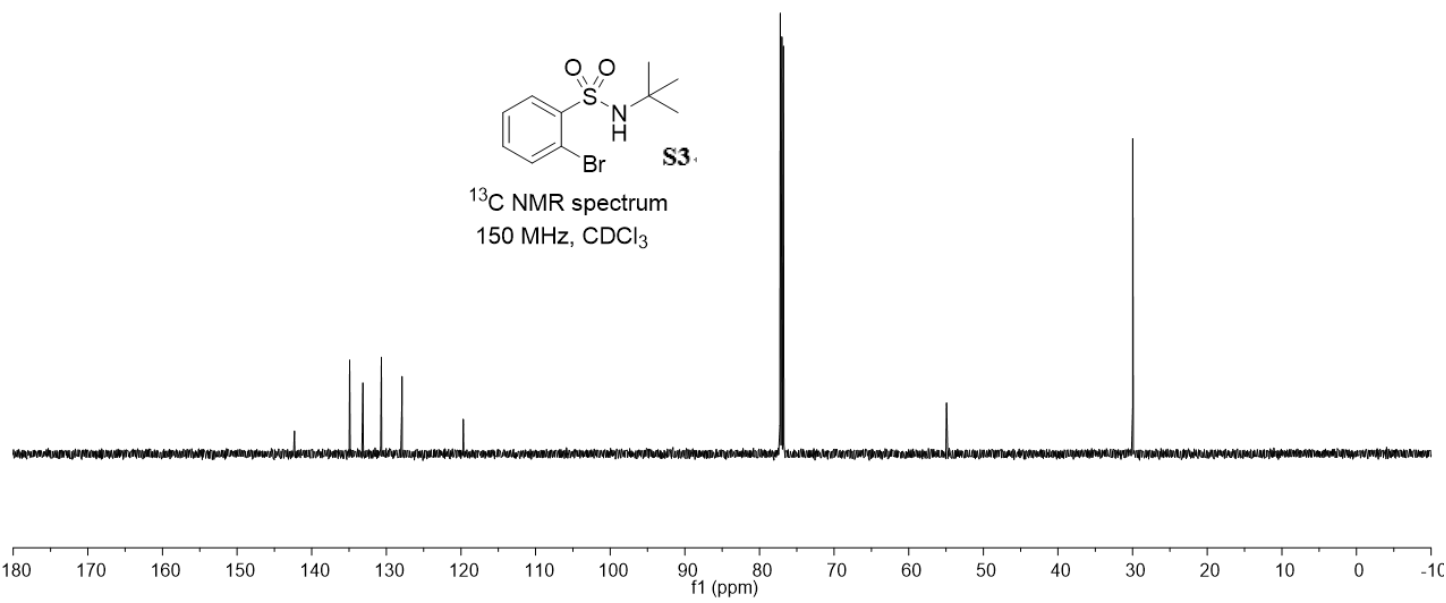



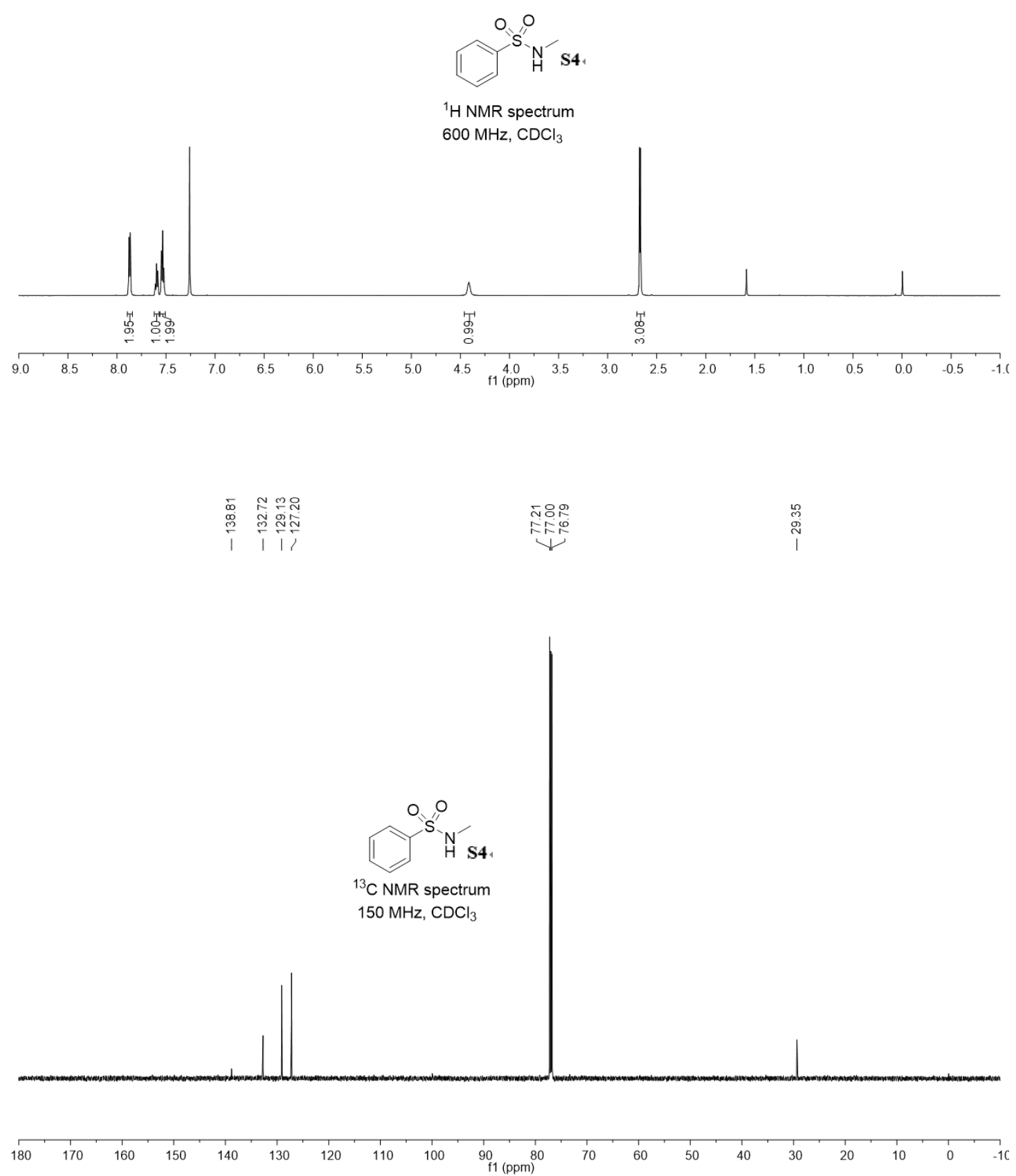

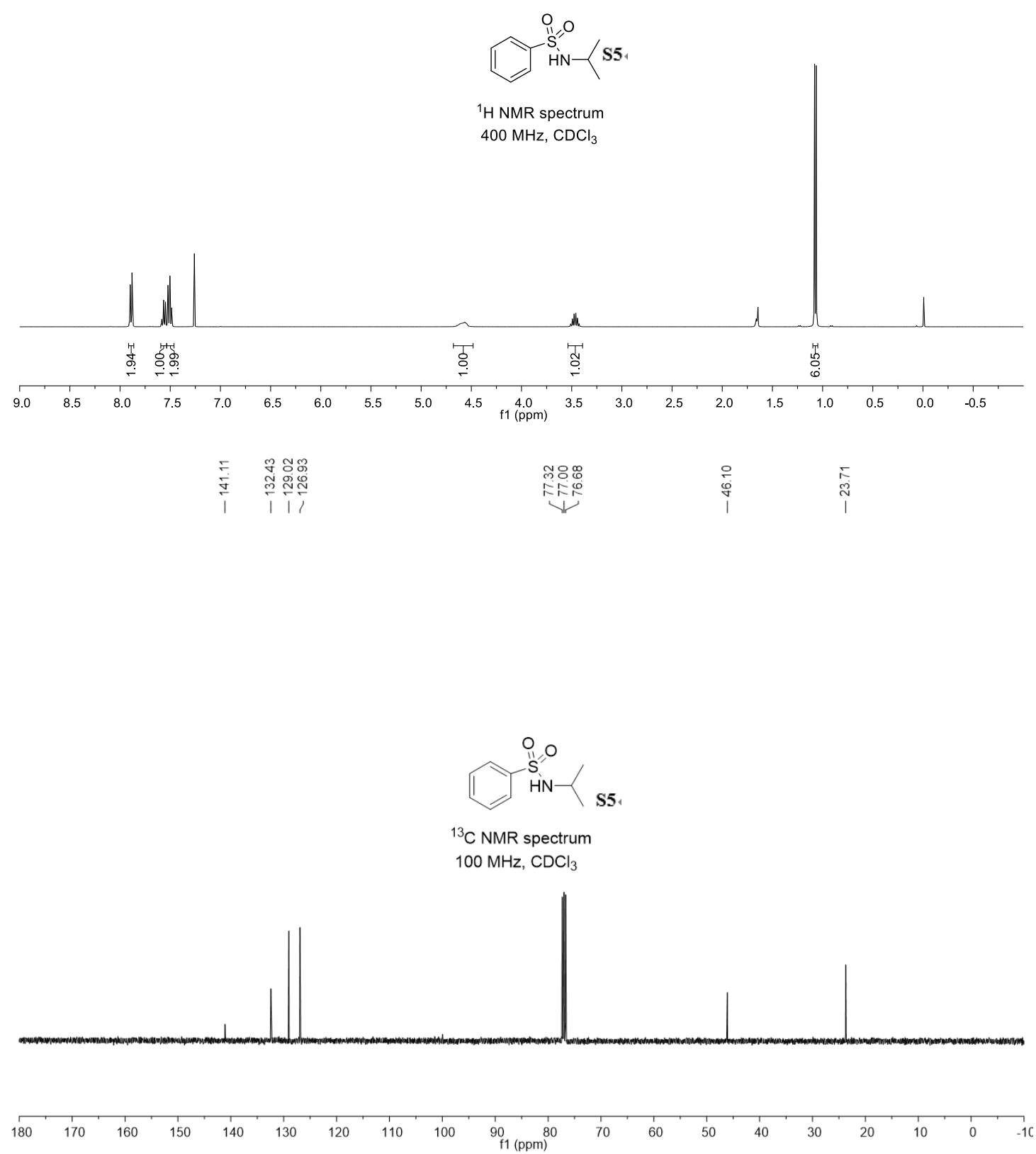


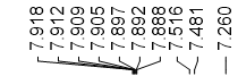

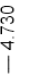

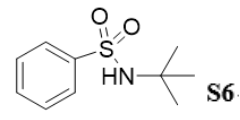

${ }^{1} \mathrm{H}$ NMR spectrum $400 \mathrm{MHz}, \mathrm{CDCl}_{3}$
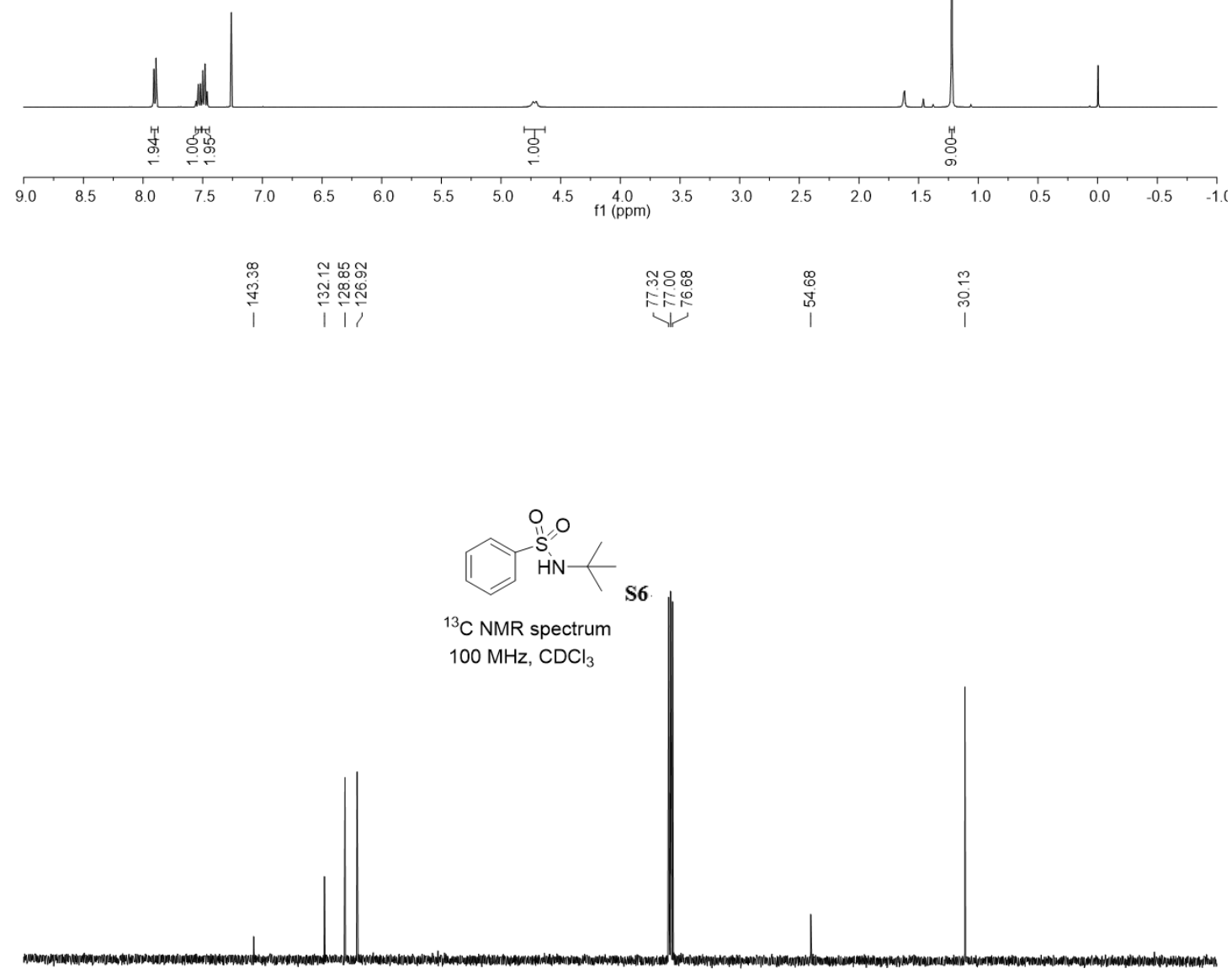

180

$\begin{array}{lllllllll}170 & 160 & 150 & 140 & 130 & 120 & 110 & 100 & 90 \\ \mathrm{f} 1(\mathrm{ppm}) & 80\end{array}$ 


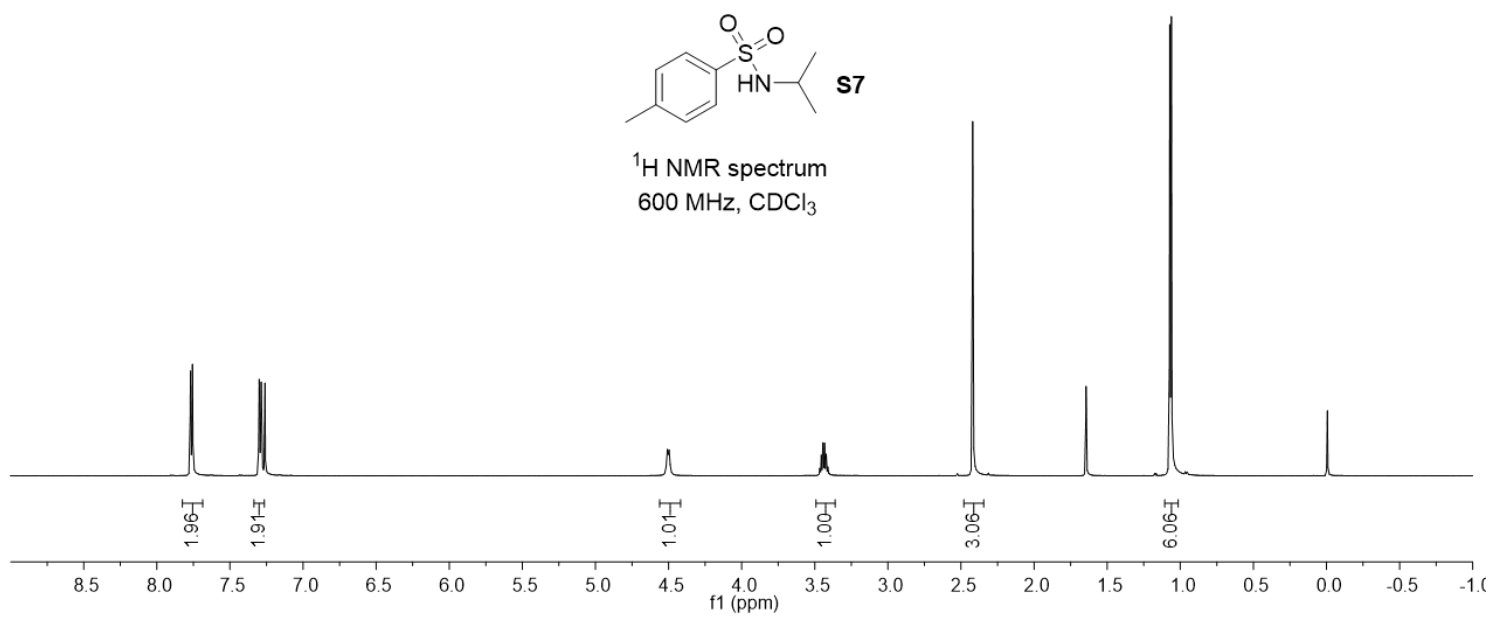

${ }^{13} \mathrm{C}$ NMR spectrum $150 \mathrm{MHz} \mathrm{CDCl}_{3}$

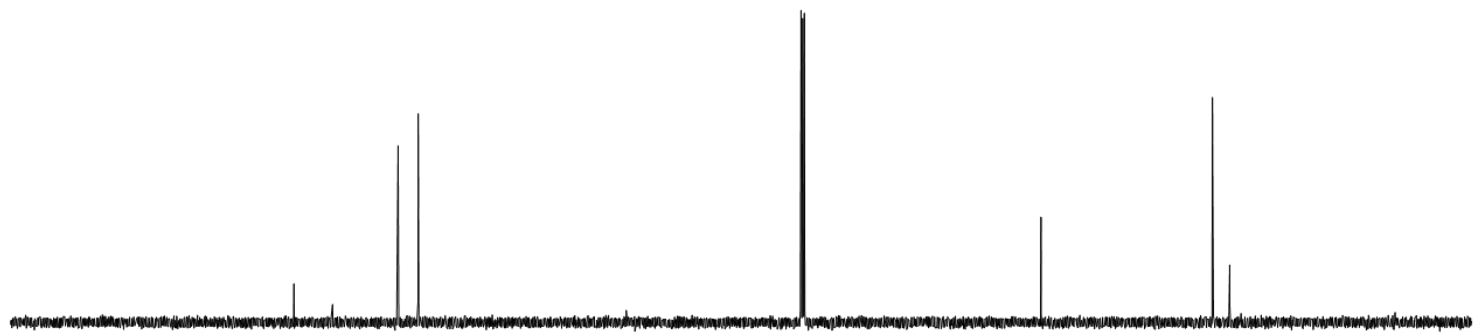

\begin{tabular}{rlllllllllllllllllllllll}
\hline 180 & 170 & 160 & 150 & 140 & 130 & 120 & 110 & 100 & 90 & 80 & 70 & 60 & 50 & 40 & 30 & 20 & 10 & 0 & -10
\end{tabular} 

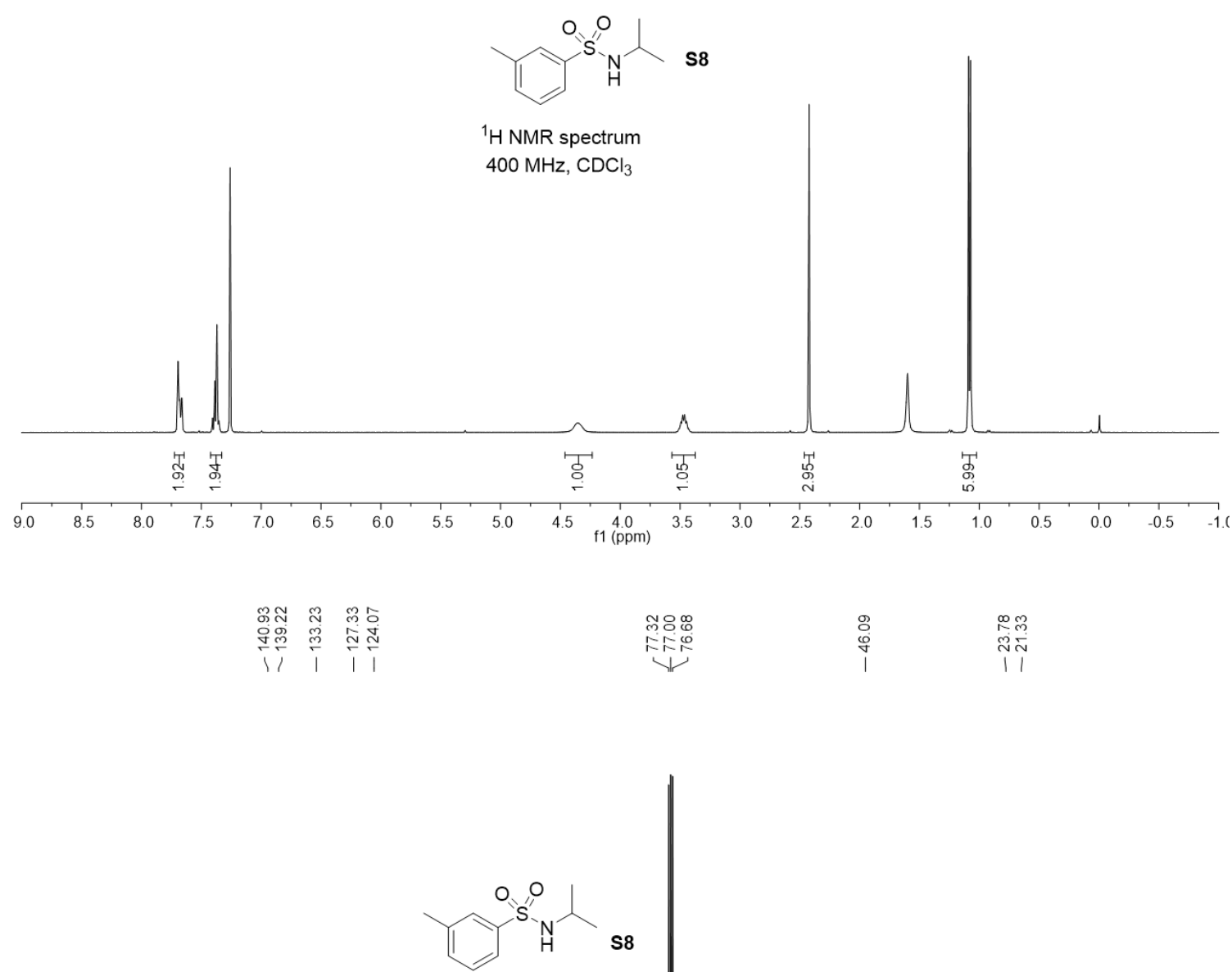

${ }^{13} \mathrm{C}$ NMR spectrum $100 \mathrm{MHz}, \mathrm{CDCl}_{3}$

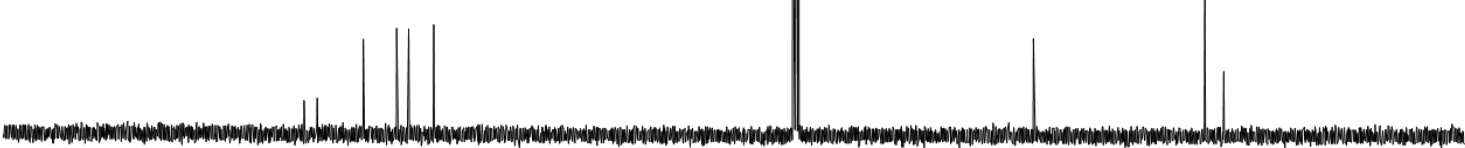

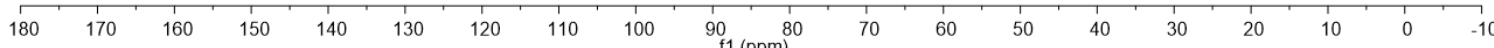




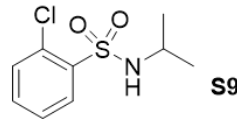

${ }^{1} \mathrm{H}$ NMR spectrum $600 \mathrm{MHz}, \mathrm{CDCl}_{3}$ s9
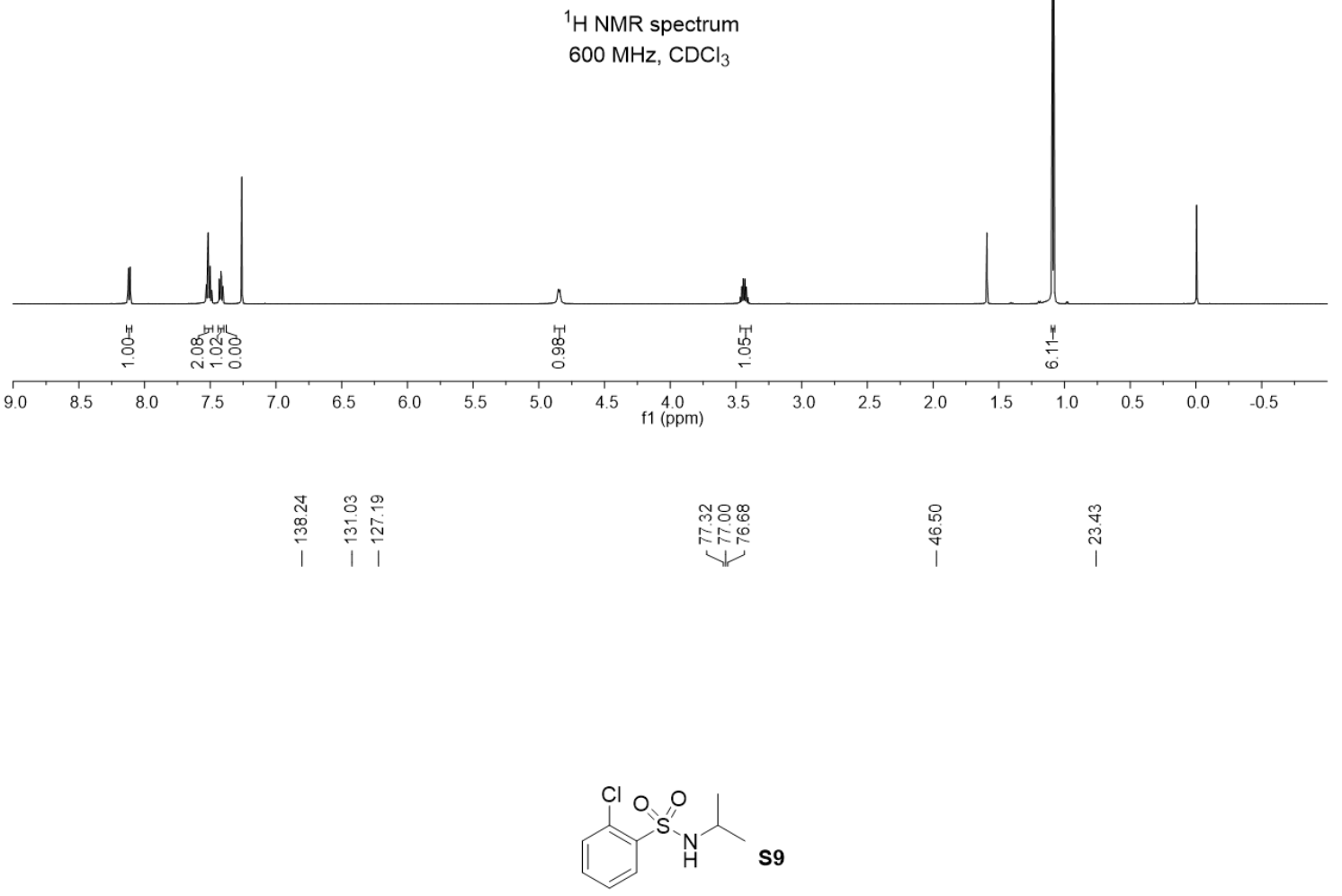

${ }^{13} \mathrm{C}$ NMR spectrum $100 \mathrm{MHz} \mathrm{CDCl}_{3}$

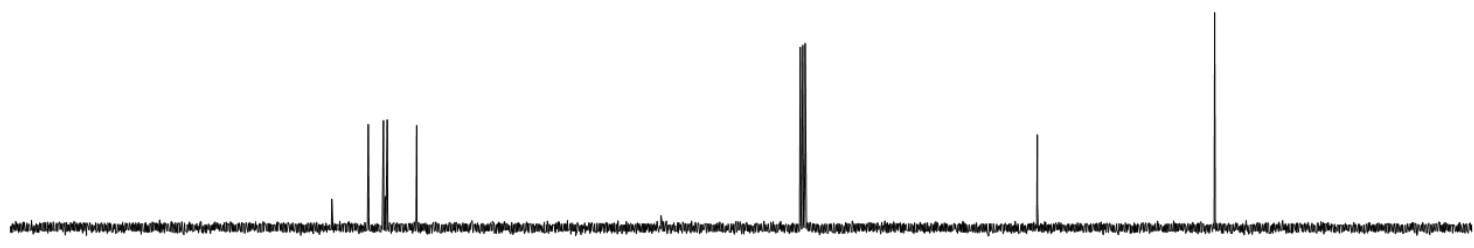

\begin{tabular}{rllllllllllllllllll}
1 & 1 \\
\hline 170 & 160 & 150 & 140 & 130 & 120 & 110 & 100 & 90 & 80 & 70 & 60 & 50 & 40 & 30 & 20 & 10 & 0 & -10
\end{tabular} 

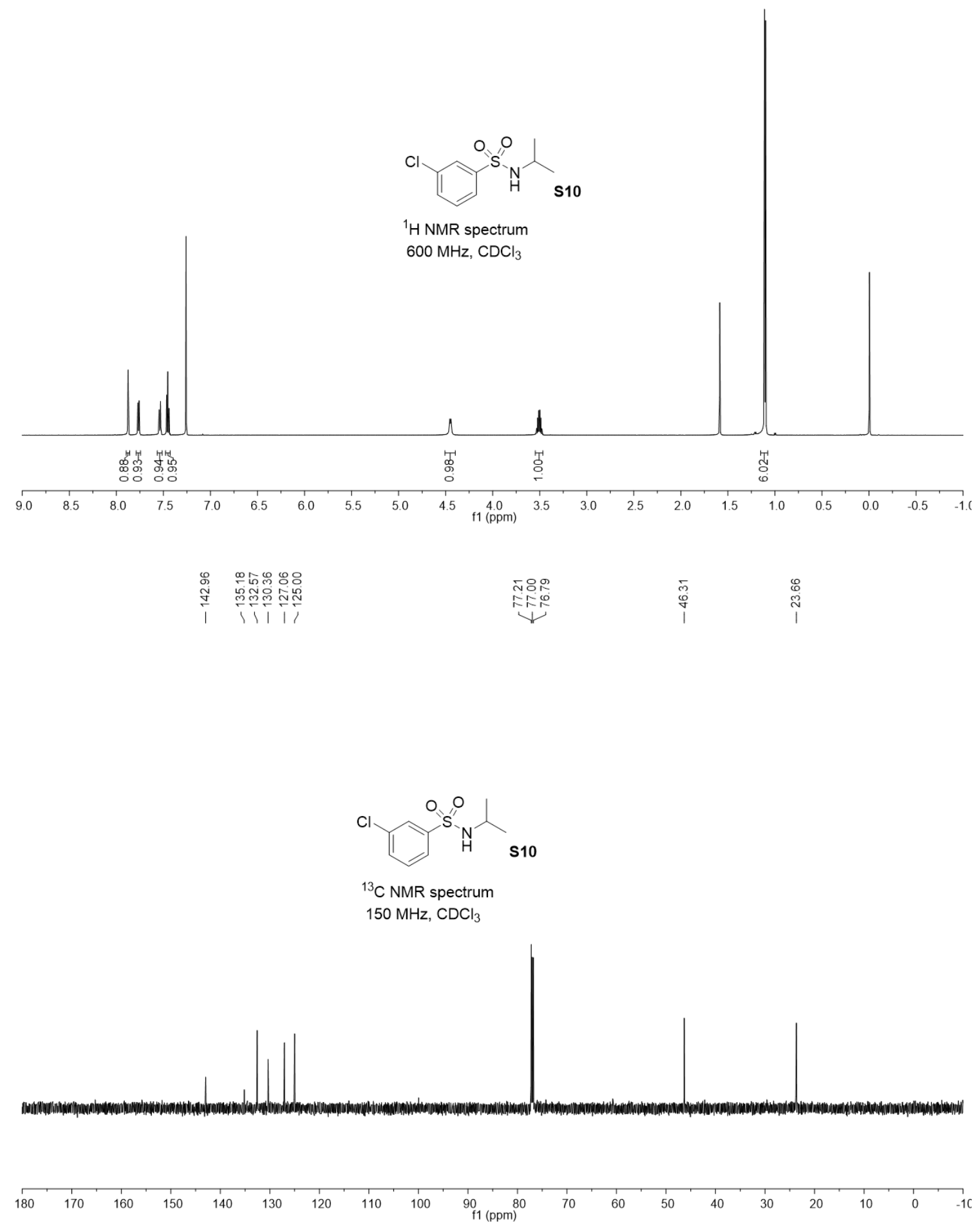


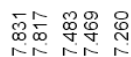

乲等

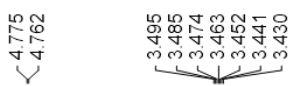

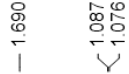

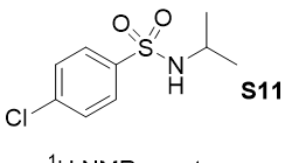

${ }^{1} \mathrm{H}$ NMR spectrum $600 \mathrm{MHz}, \mathrm{CDCl}_{3}$

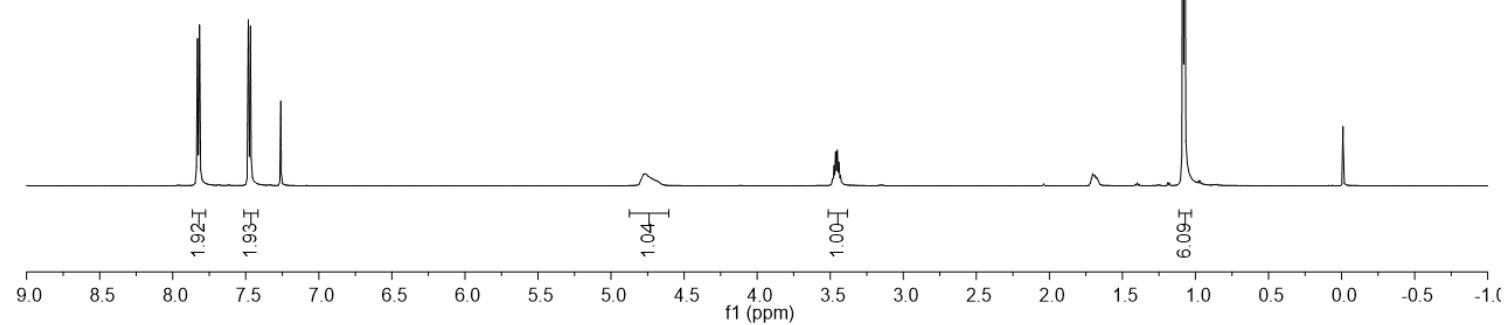

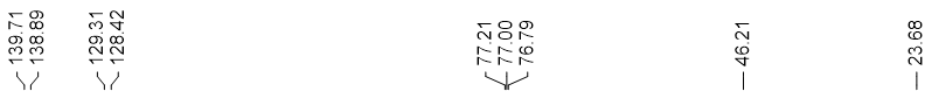

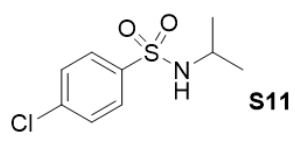

${ }^{13} \mathrm{C}$ NMR spectrum

$150 \mathrm{MHz}, \mathrm{CDCl}_{3}$

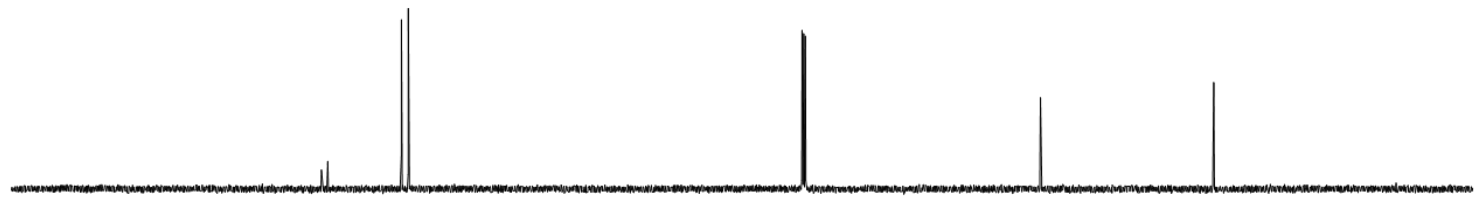

$\begin{array}{llllllllllllllllllll}180 & 170 & 160 & 150 & 140 & 130 & 120 & 110 & 100 & 90 & 80 & 70 & 60 & 50 & 40 & 30 & 20 & 10 & 0 & -10\end{array}$ 


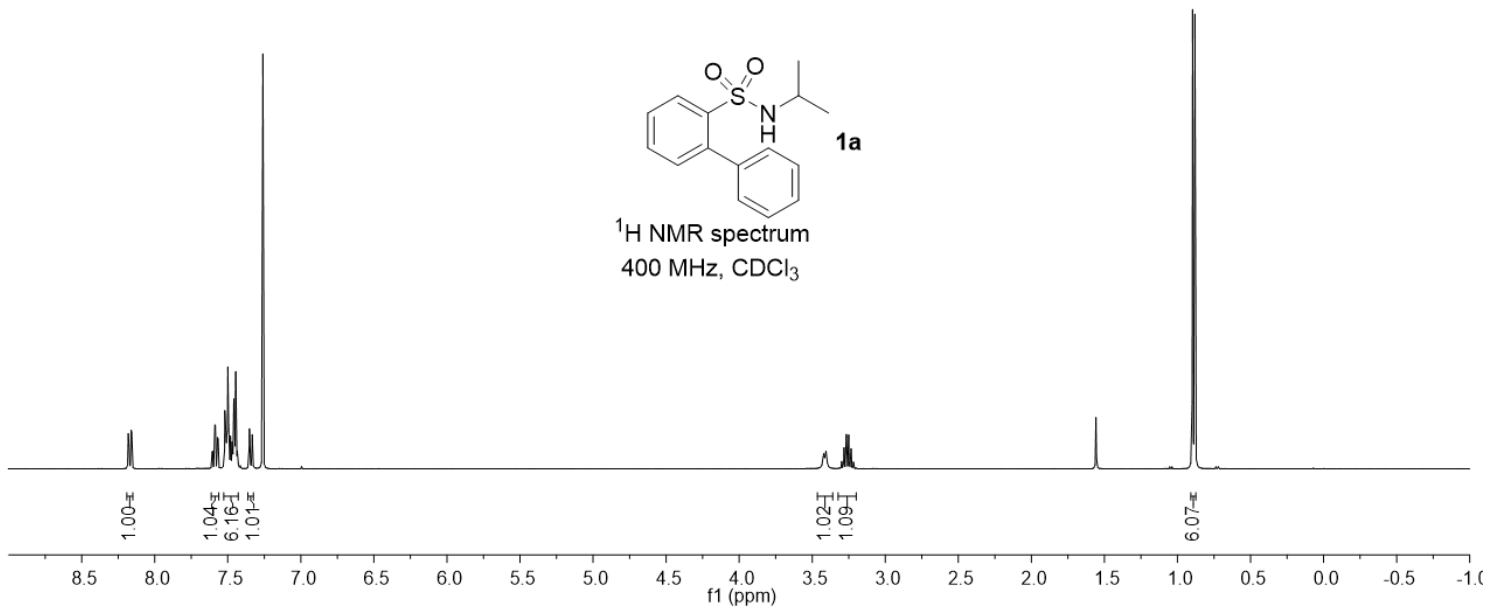

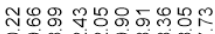

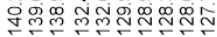

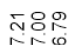

NÁ

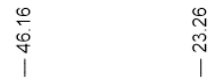

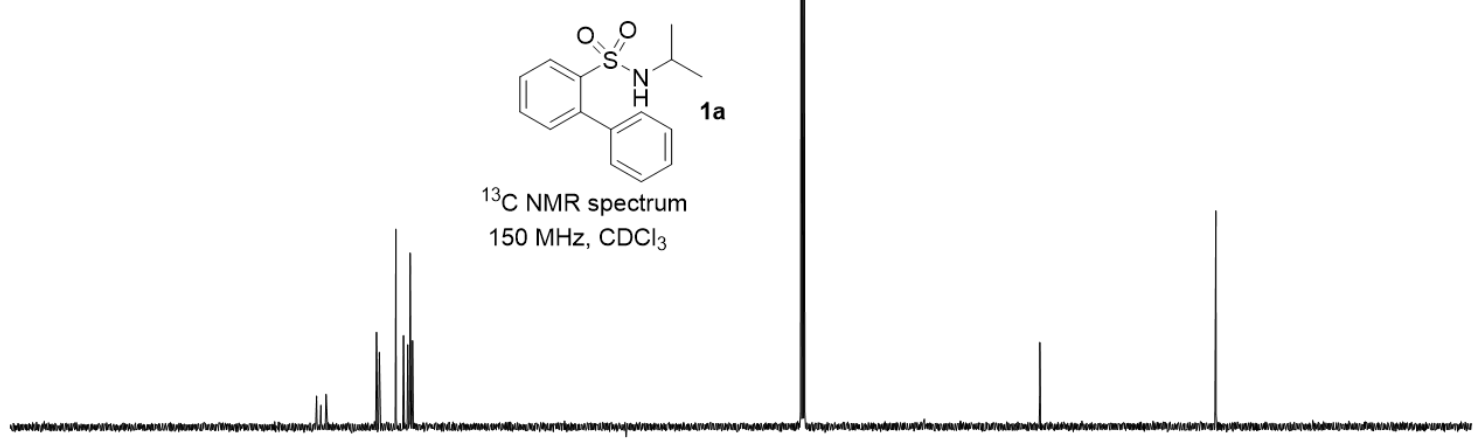

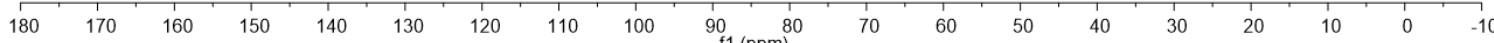


1)

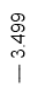

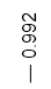

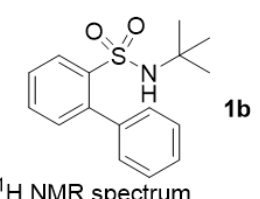

$1 b$

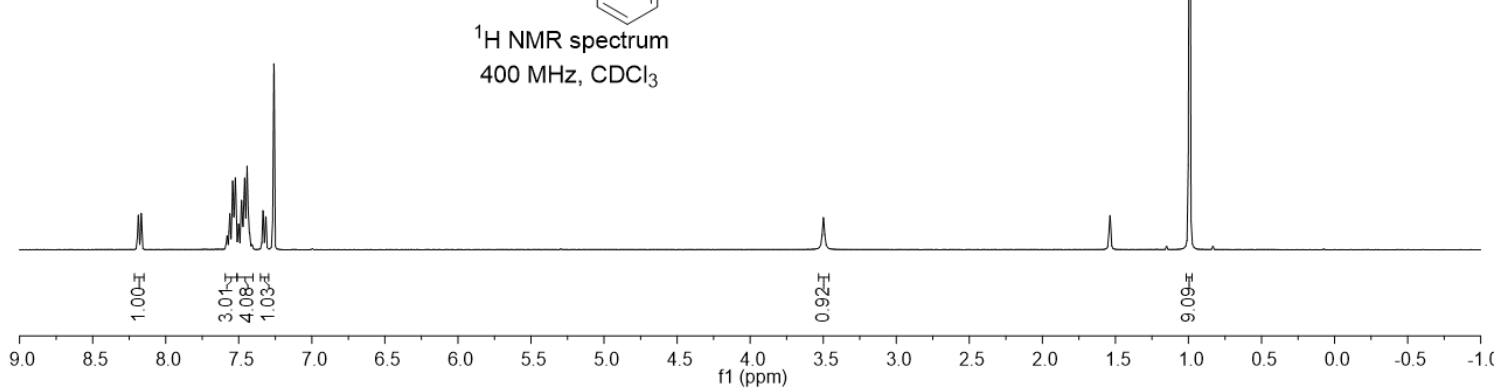

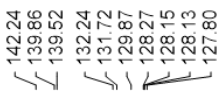

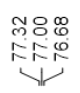

焉

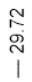

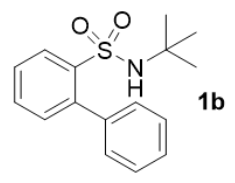

${ }^{13} \mathrm{C}$ NMR spectrum $100 \mathrm{MHz} \mathrm{CDCl}_{3}$
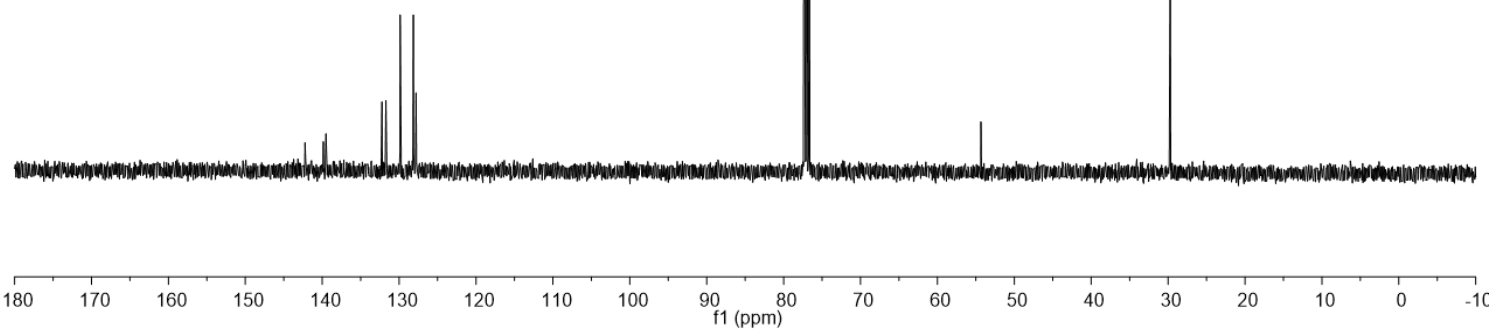

66 


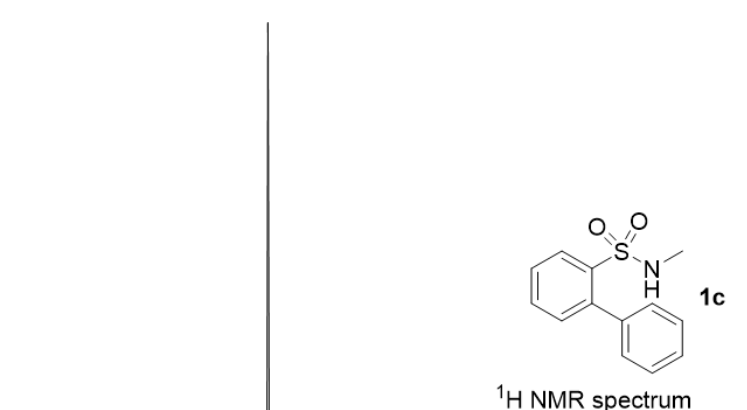

$400 \mathrm{MHz}, \mathrm{CDCl}_{3}$
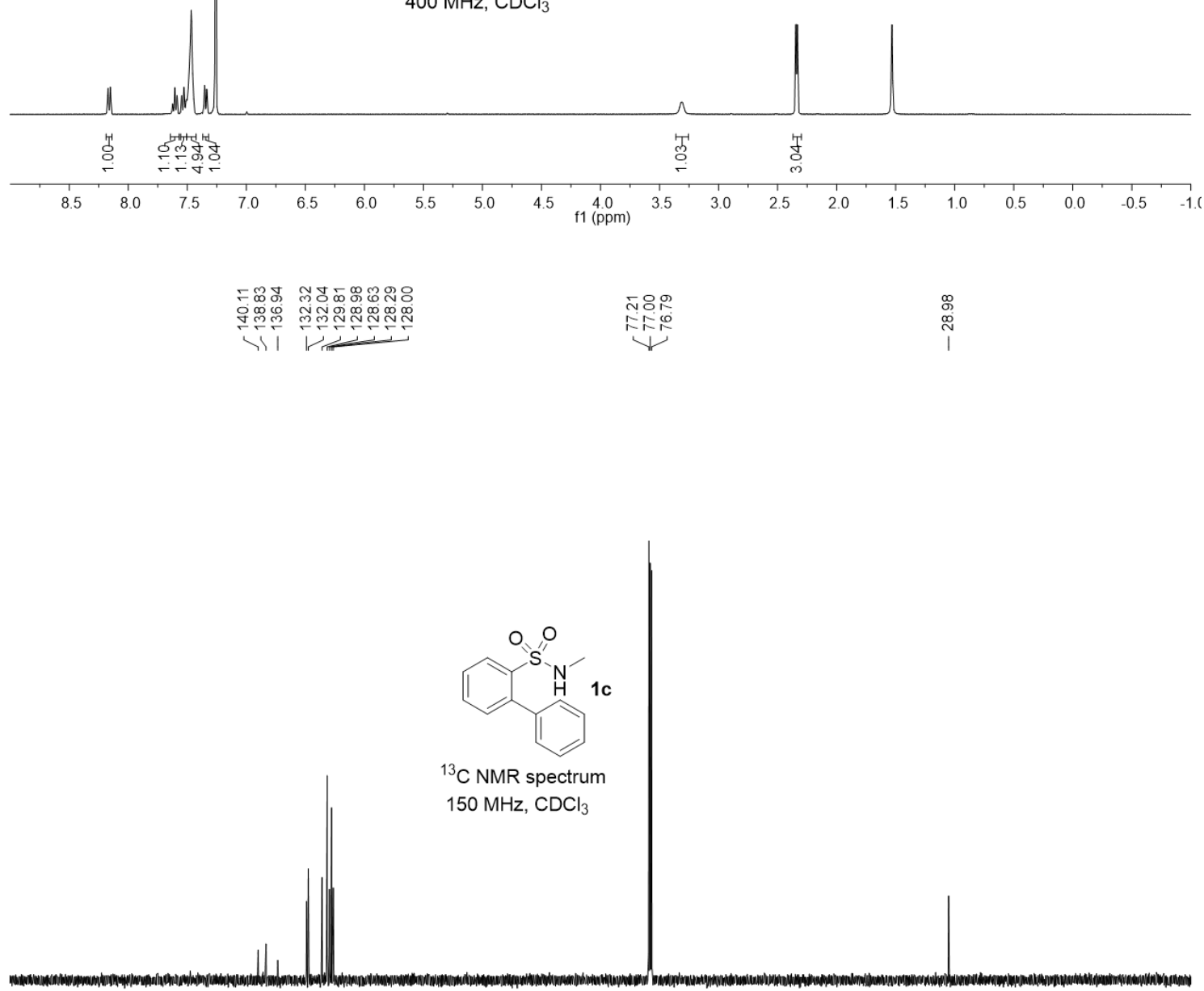

\begin{tabular}{llllllllll}
180 & 170 & 160 & 150 & 140 & 130 & 120 & 110 & 100 & 100 \\
\hline $\mathrm{f} 1(\mathrm{ppm})$ & 100
\end{tabular} 


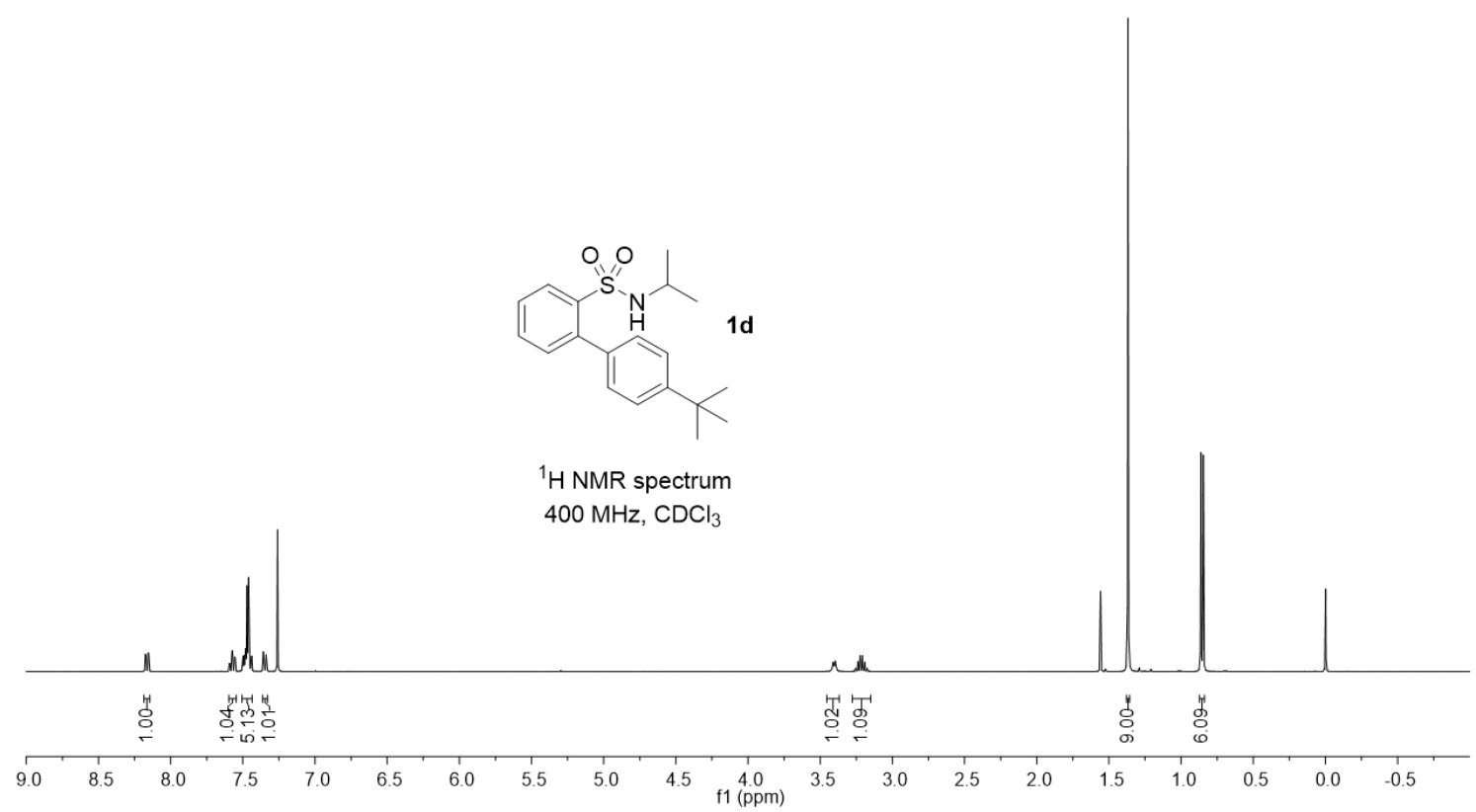

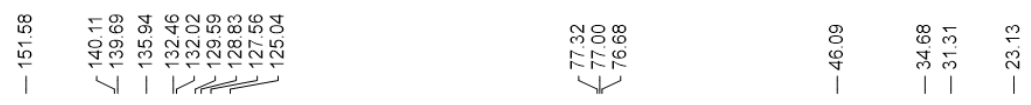

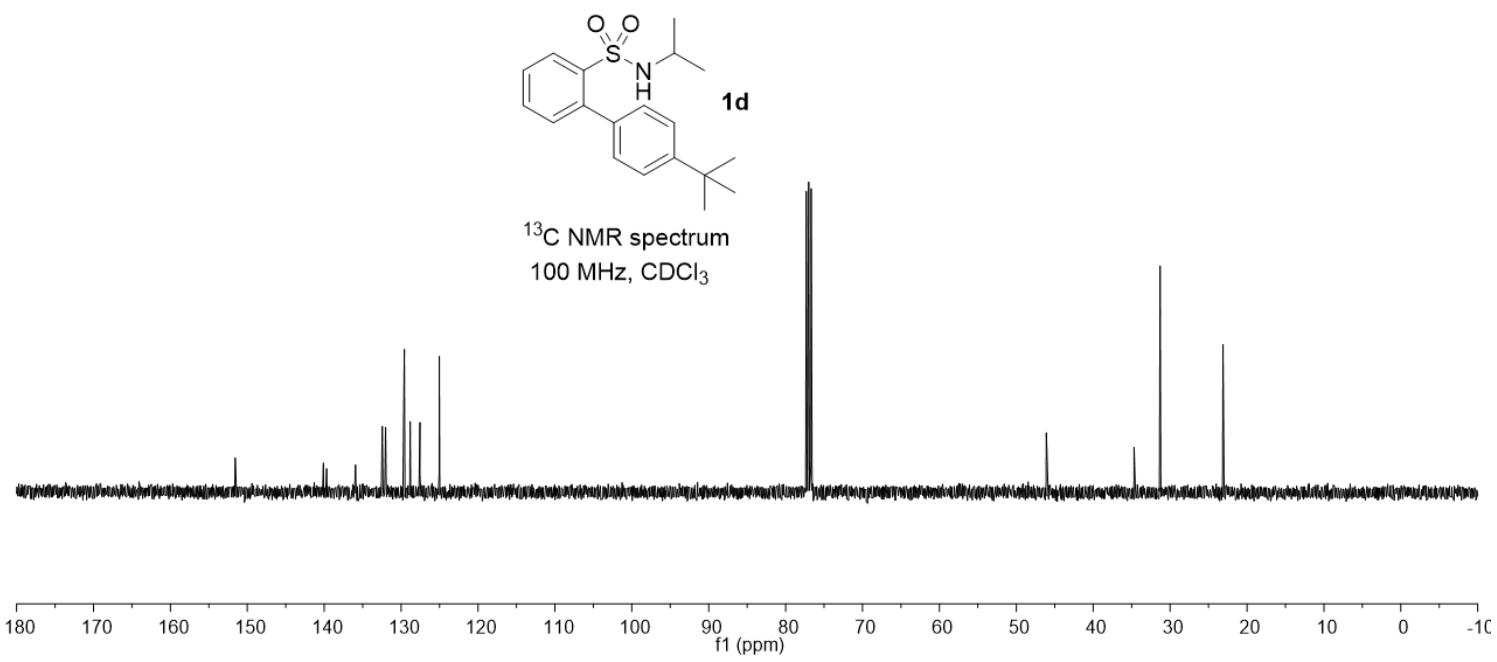




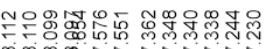

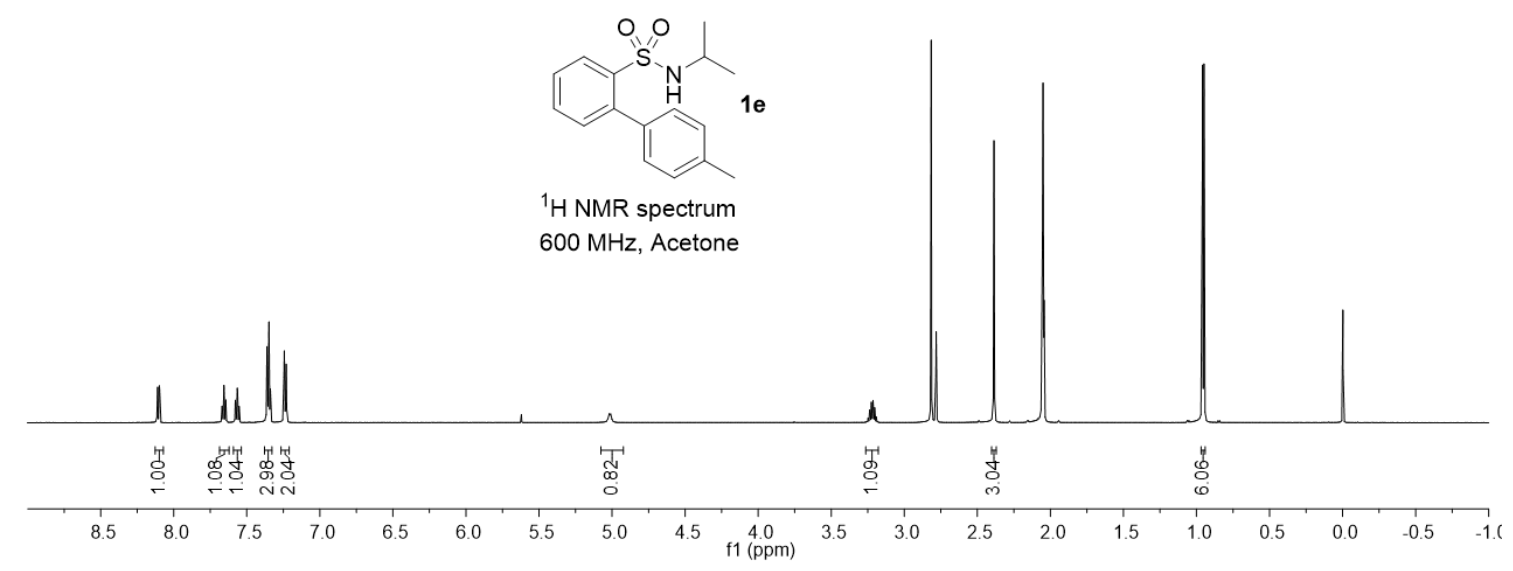

융유융듀

ดुำ

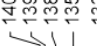

No⿱

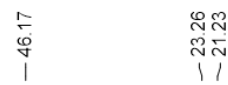

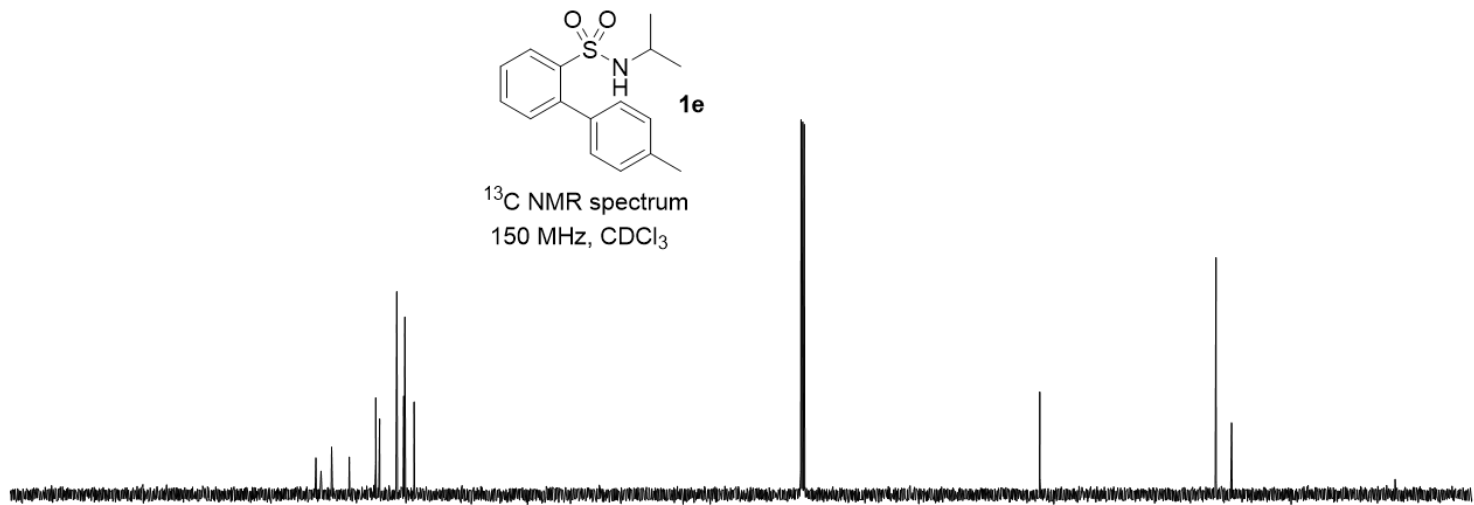

\begin{tabular}{llllllllllllllllllll}
\hline 170 & 160 & 150 & 140 & 130 & 120 & 110 & 100 & 90 & 80 & 70 & 60 & 50 & 40 & 30 & 20 & 10 & 0 & -10
\end{tabular} 


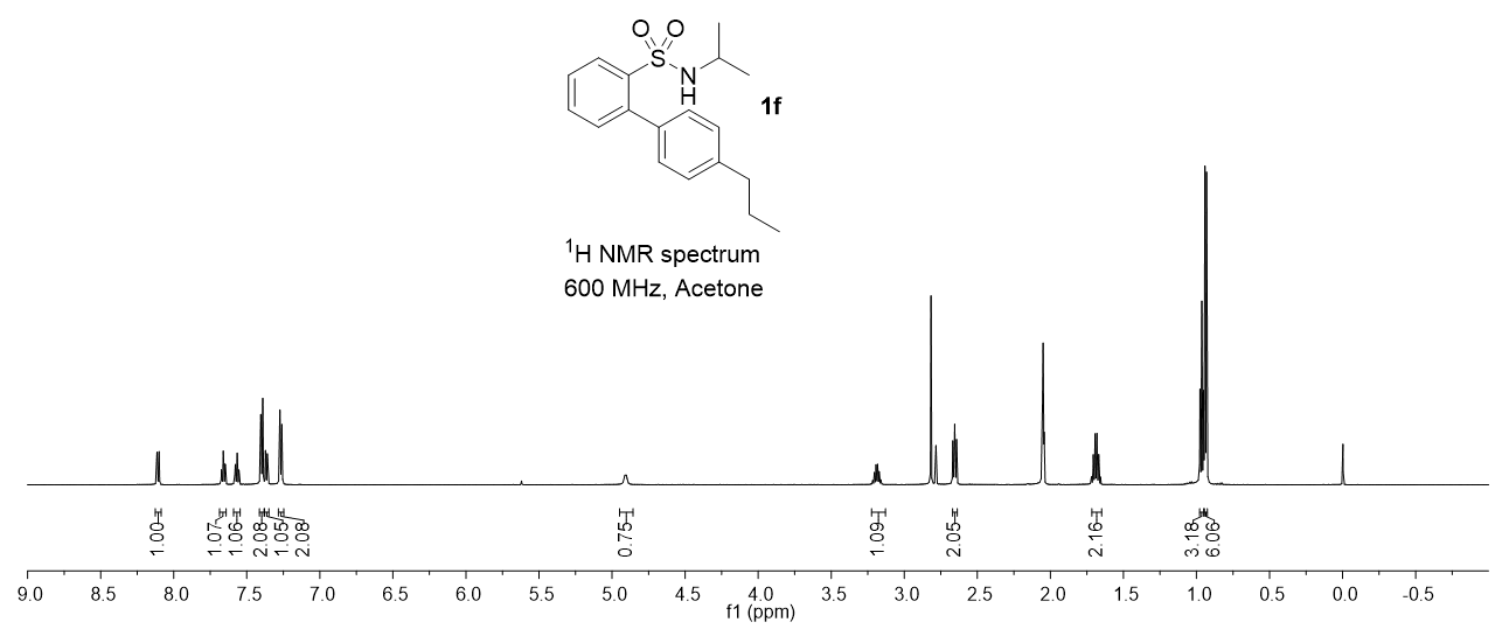

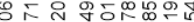

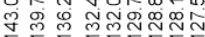

1 i i

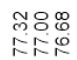

쇼

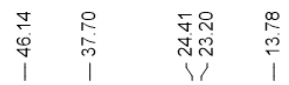

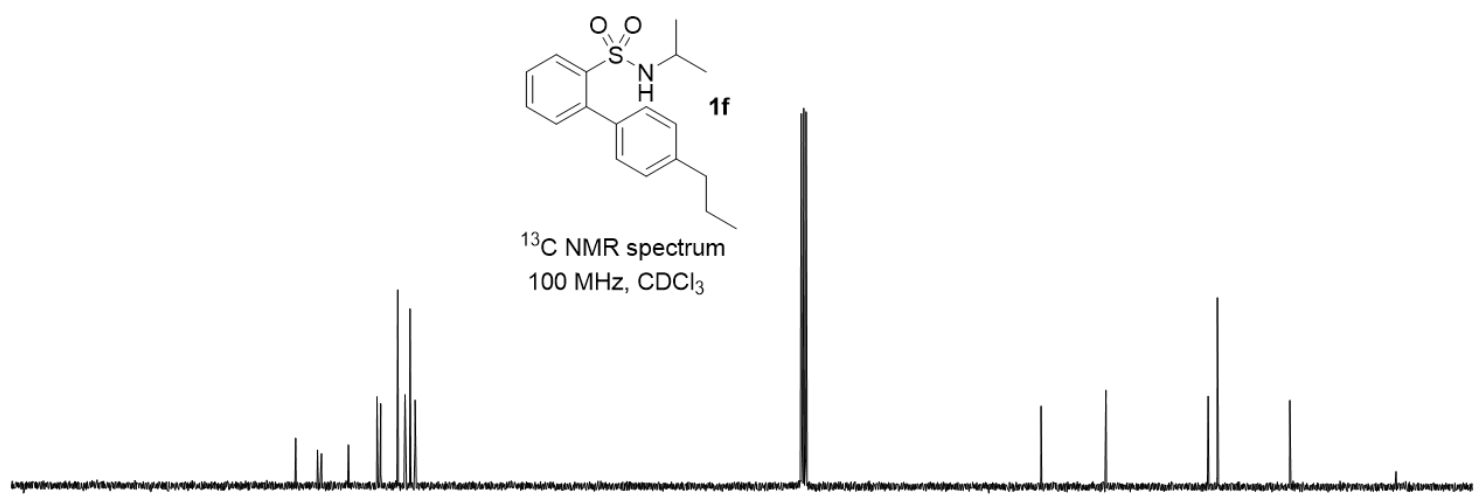

\begin{tabular}{rl|lllllllllllllllllllll}
\hline 180 & 170 & 160 & 150 & 140 & 130 & 120 & 110 & 100 & 90 & 80 & 70 & 60 & 50 & 40 & 30 & 20 & 10 & 0 & -10
\end{tabular} 


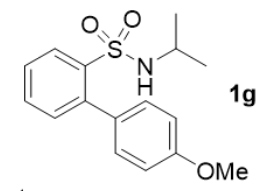

${ }^{1} \mathrm{H}$ NMR spectrum $400 \mathrm{MHz}, \mathrm{CDCl}_{3}$

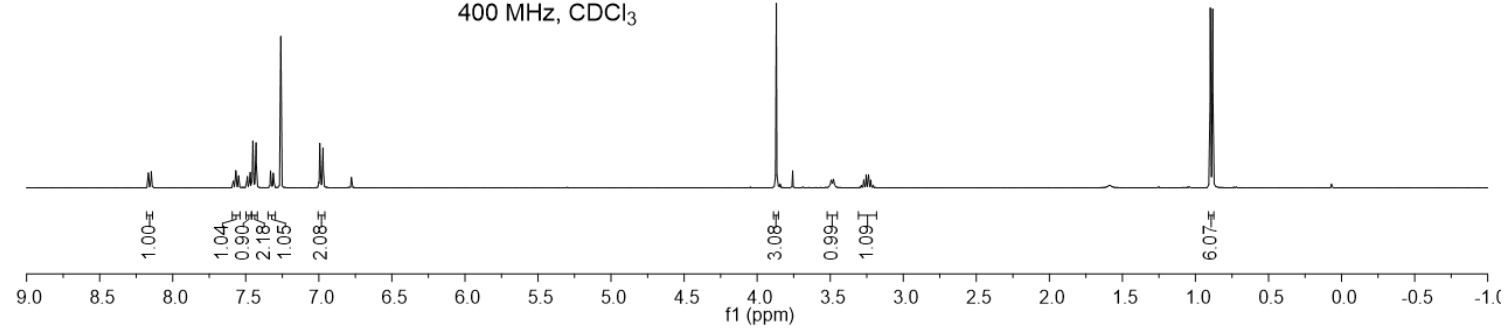

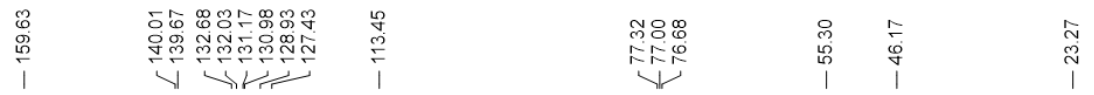

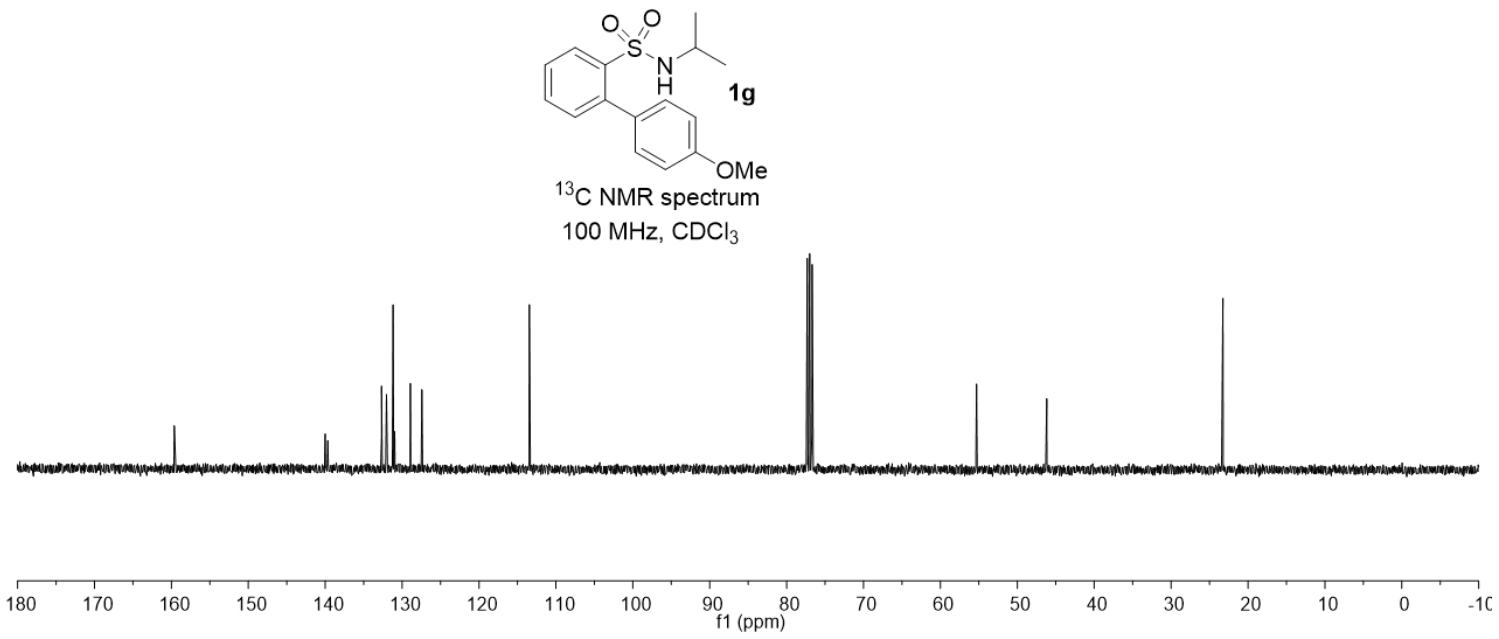



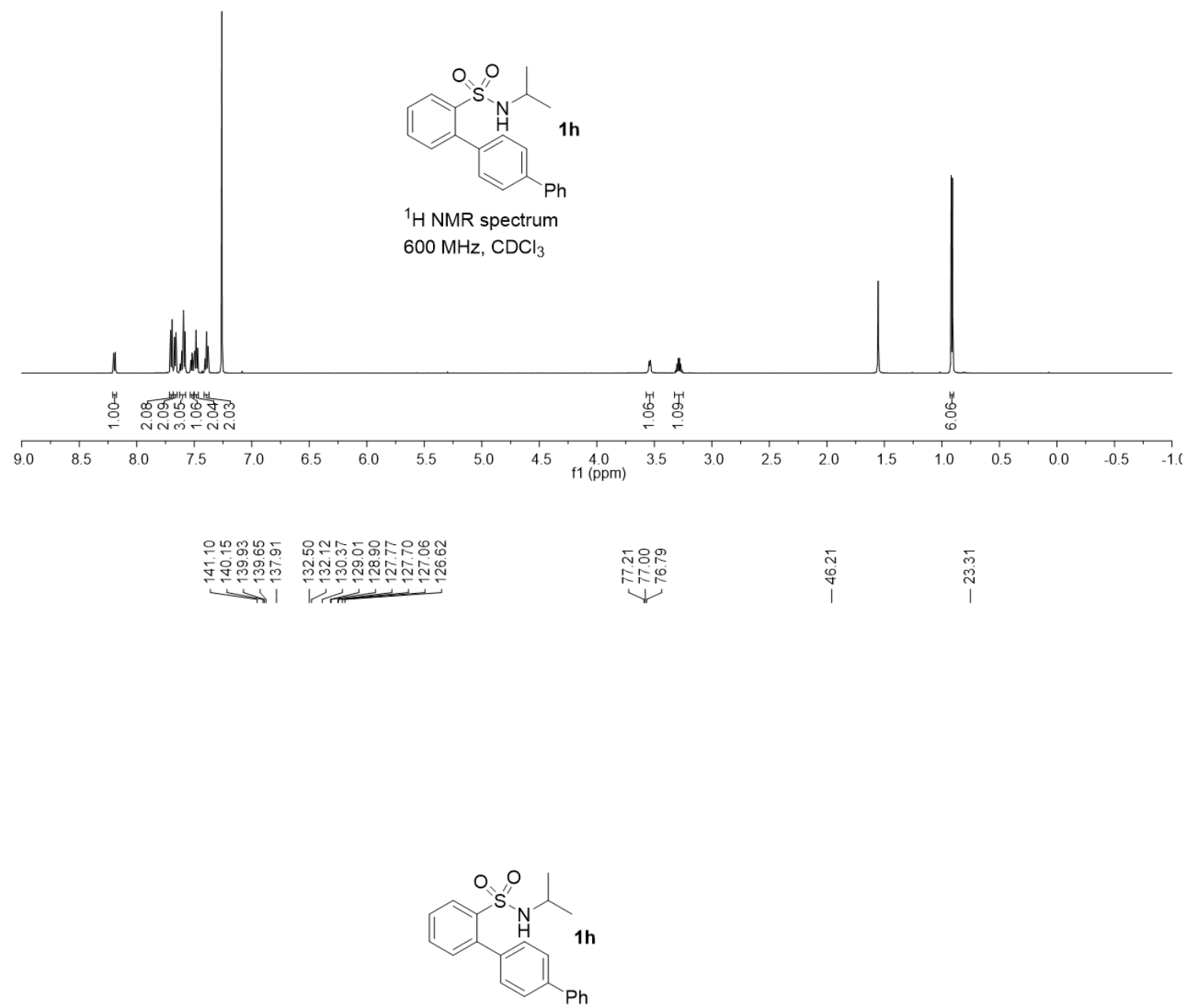

${ }^{13} \mathrm{C}$ NMR spectrum

$150 \mathrm{MHz}, \mathrm{CDCl}_{3}$

11

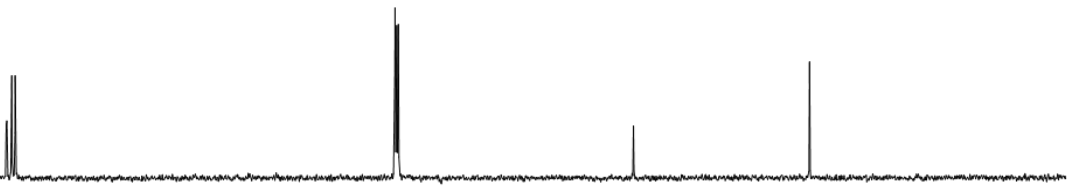

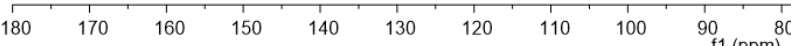




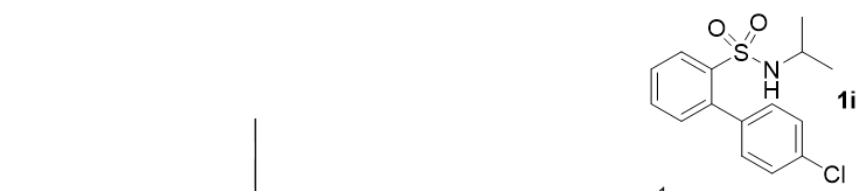

${ }^{1} \mathrm{H}$ NMR spectrum $400 \mathrm{MHz}, \mathrm{CDCl}_{3}$
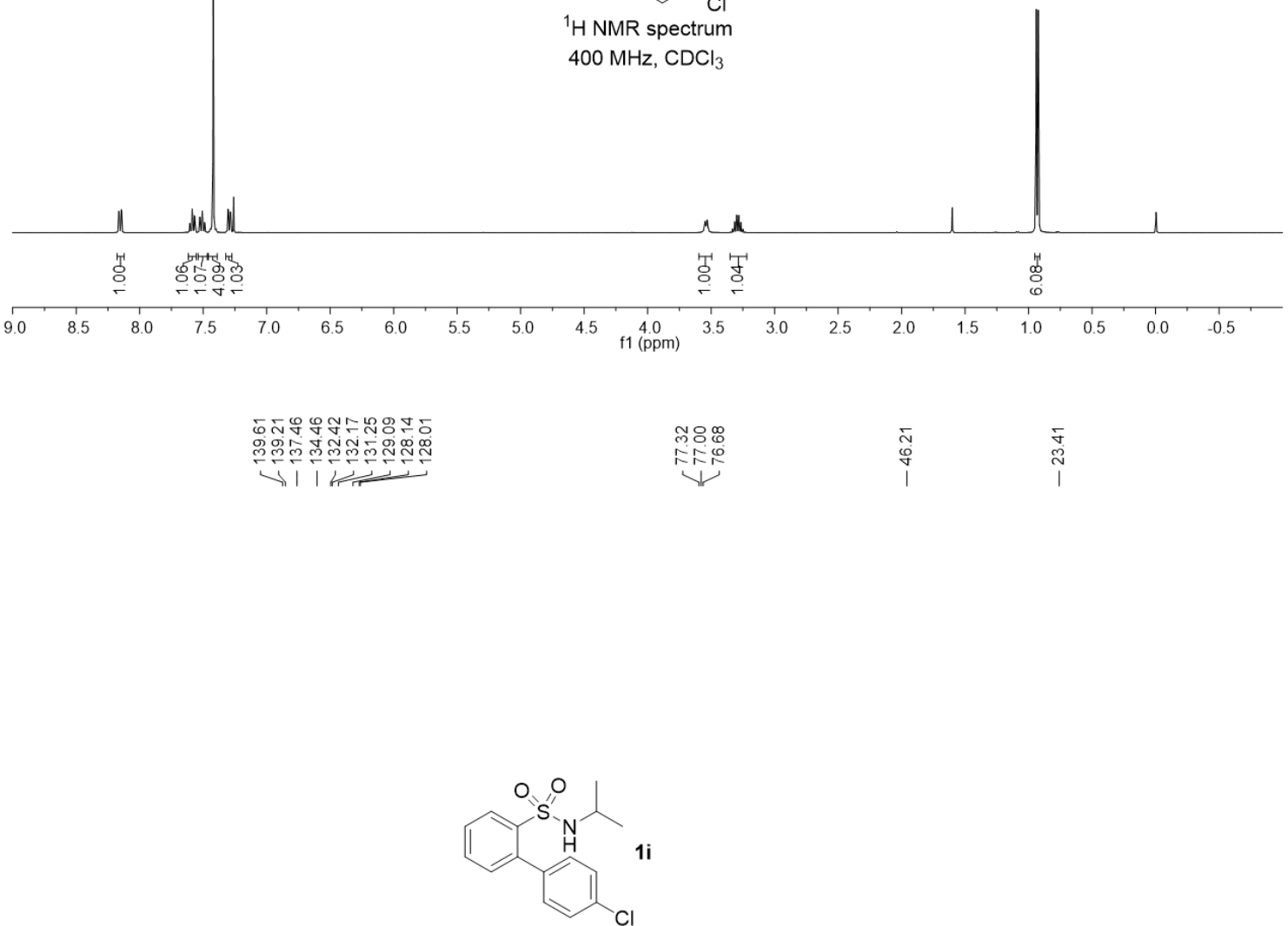

${ }^{13} \mathrm{C}$ NMR spectrum

$100 \mathrm{MHz}, \mathrm{CDCl}_{3}$

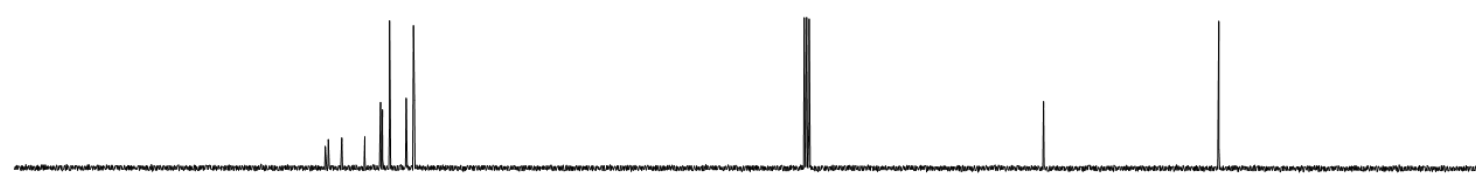

180

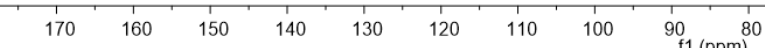




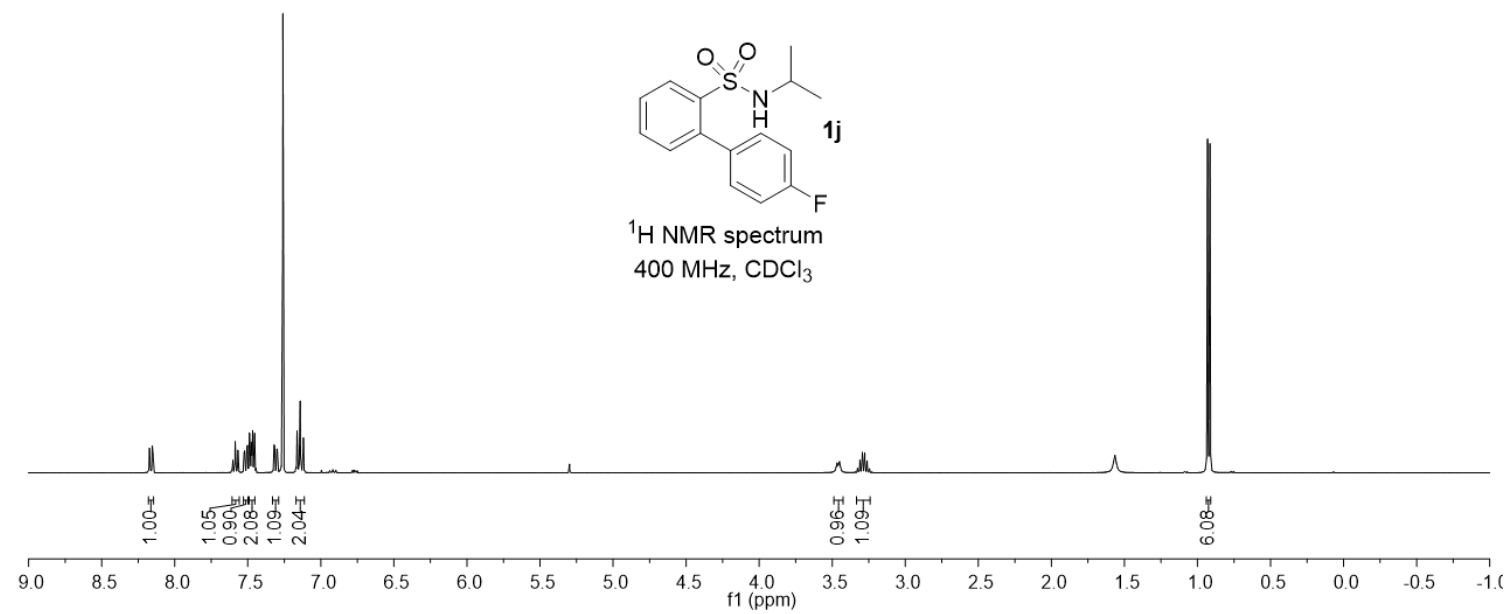

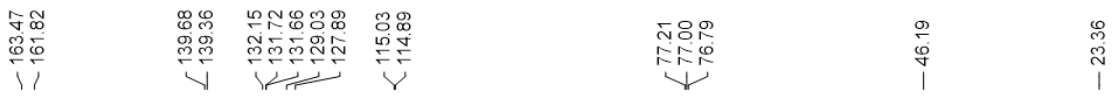

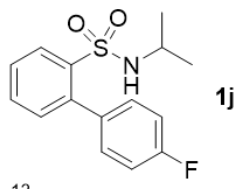

${ }^{3} \mathrm{C}$ NMR spectrum

$150 \mathrm{MHz}, \mathrm{CDCl}_{3}$

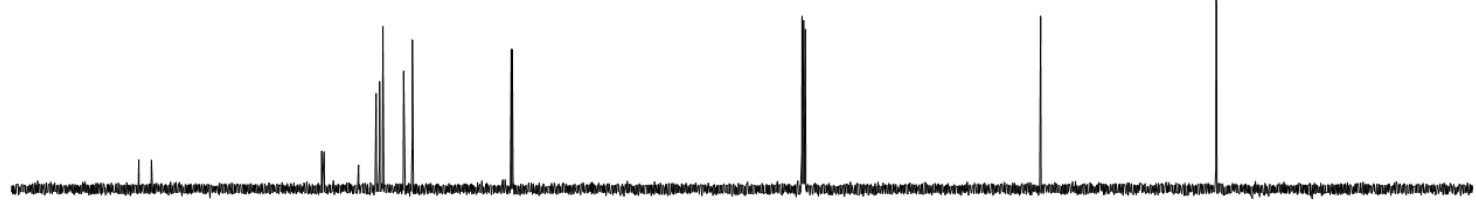

$\begin{array}{llllllllllllllllllll}180 & 170 & 160 & 150 & 140 & 130 & 120 & 110 & 100 & 90 & 80 & 70 & 60 & 50 & 40 & 30 & 20 & 10 & 0 & -10\end{array}$ 


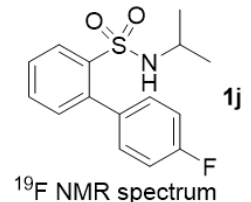

$375 \mathrm{MHz}, \mathrm{CDCl}_{3}$

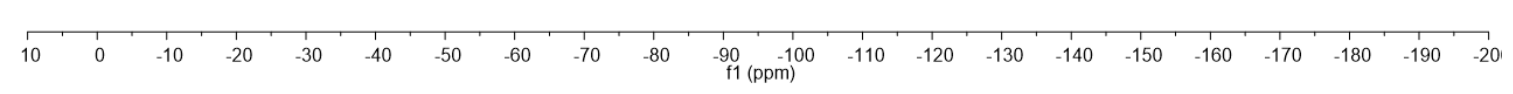

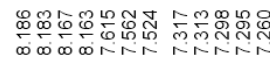

|

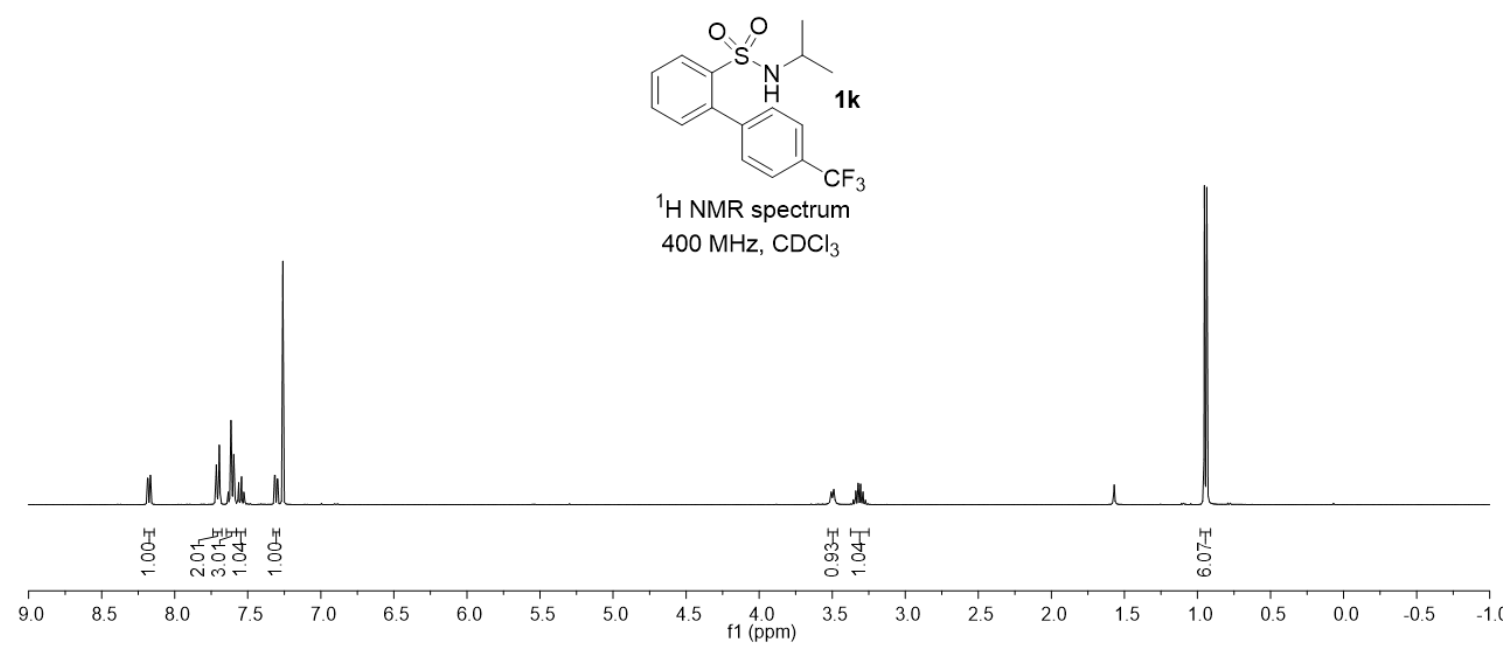



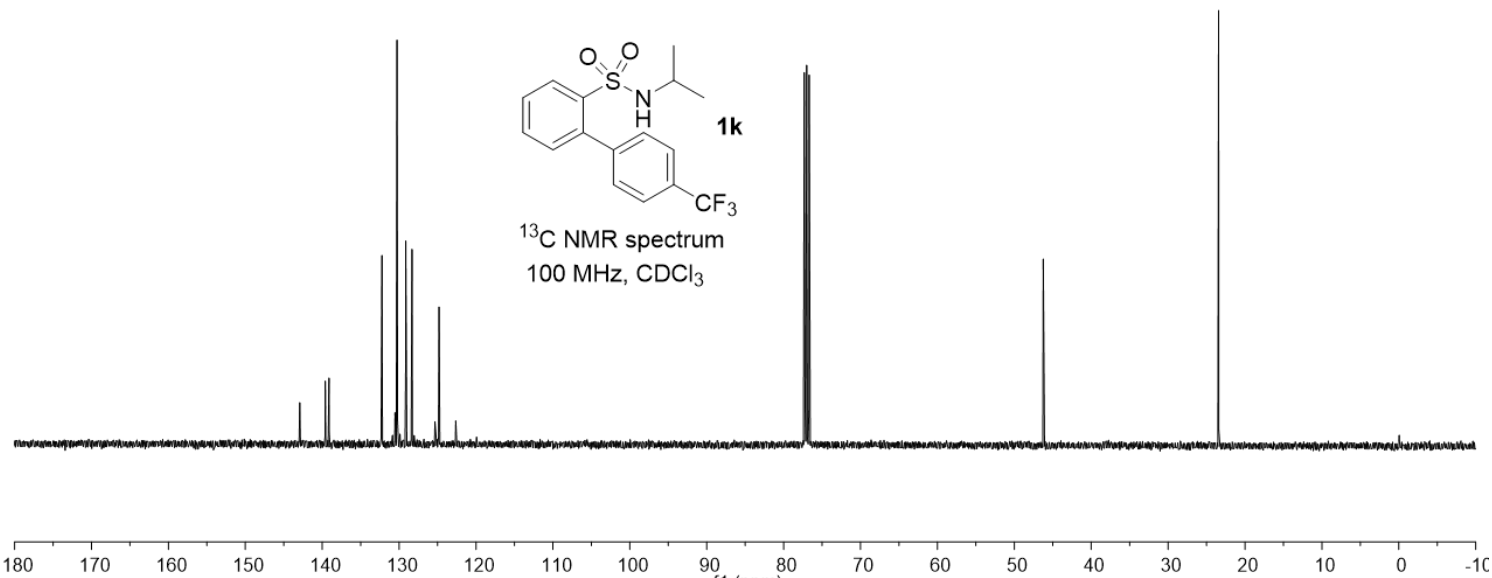

f1 (ppm)

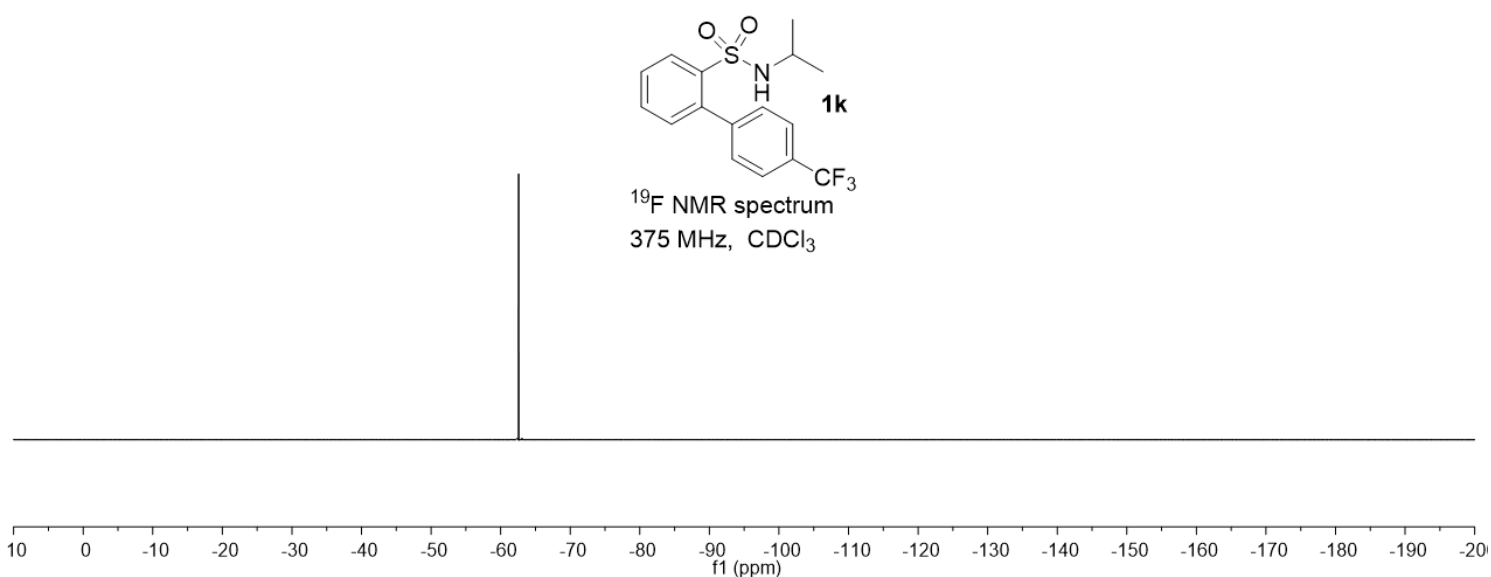



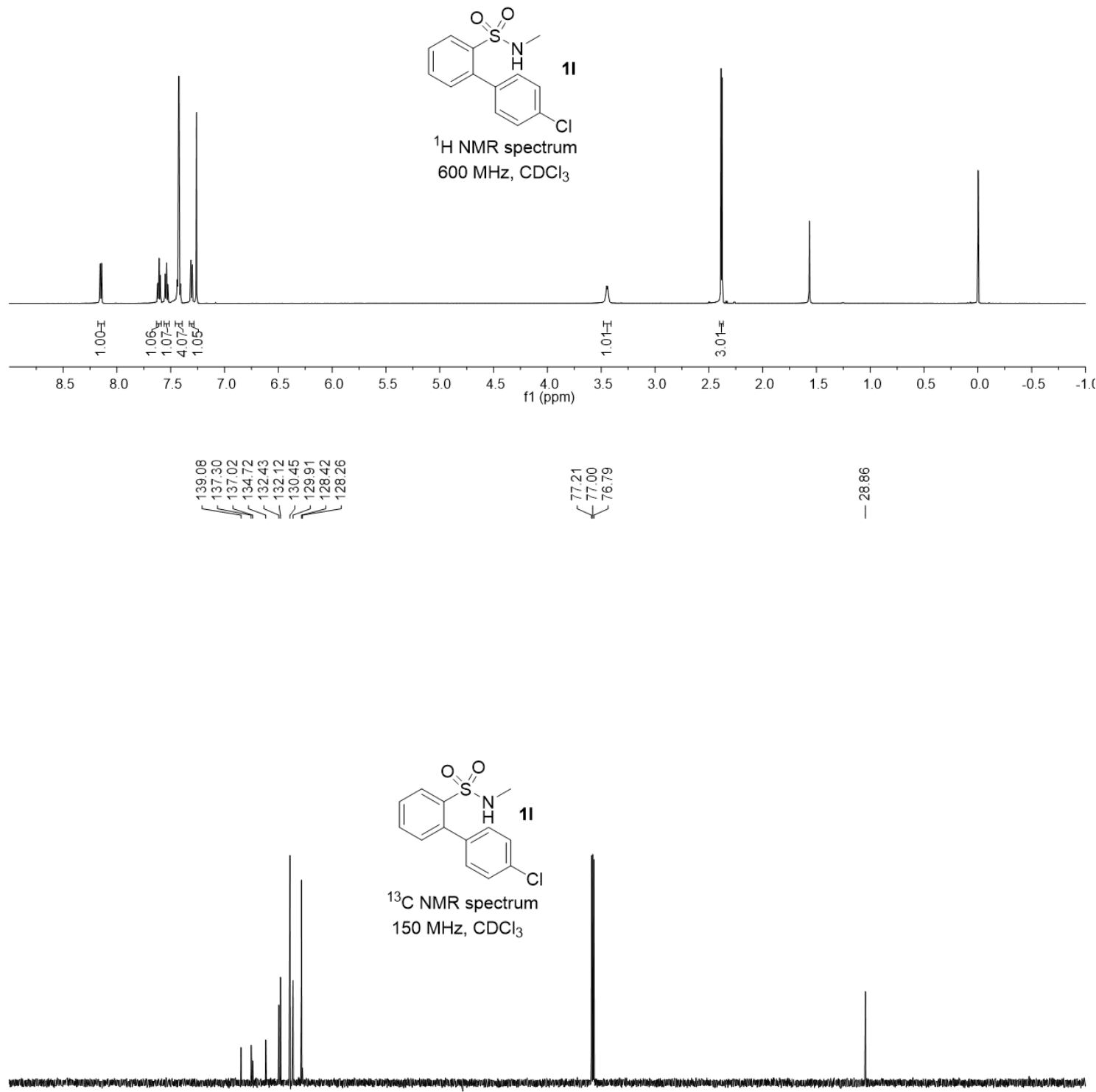

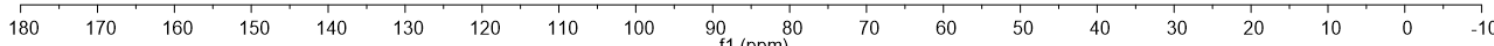




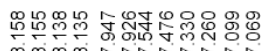

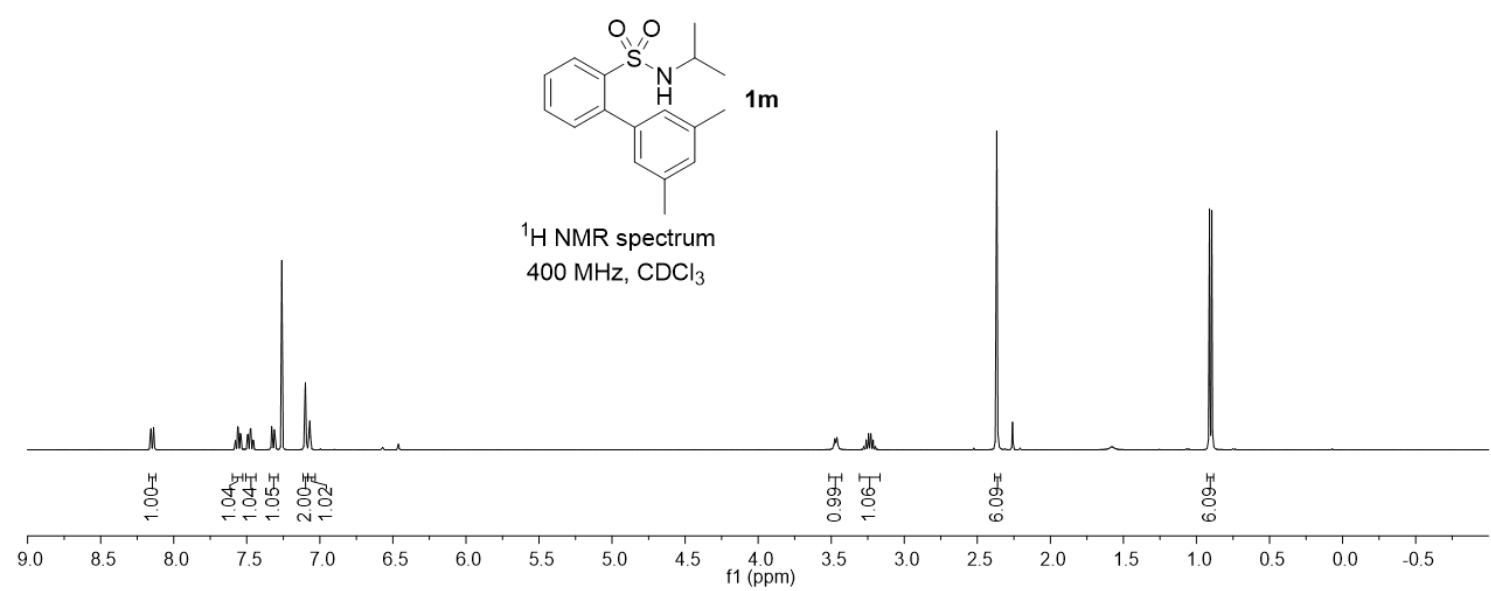

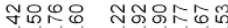

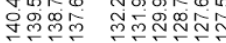

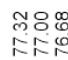

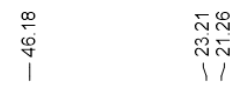

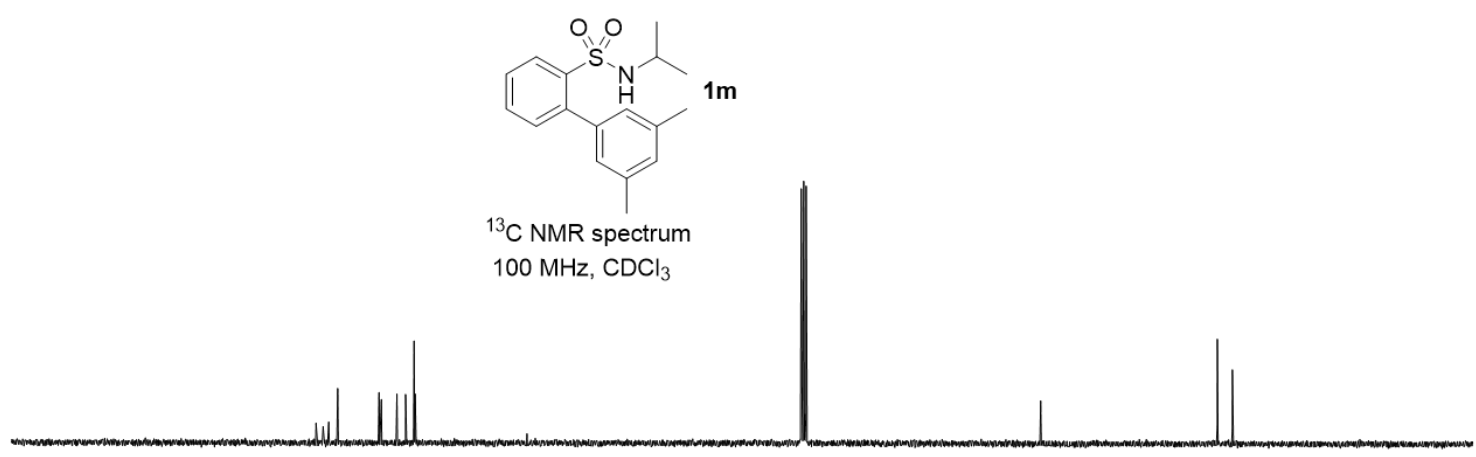

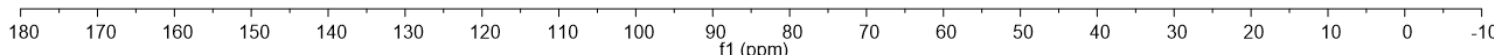



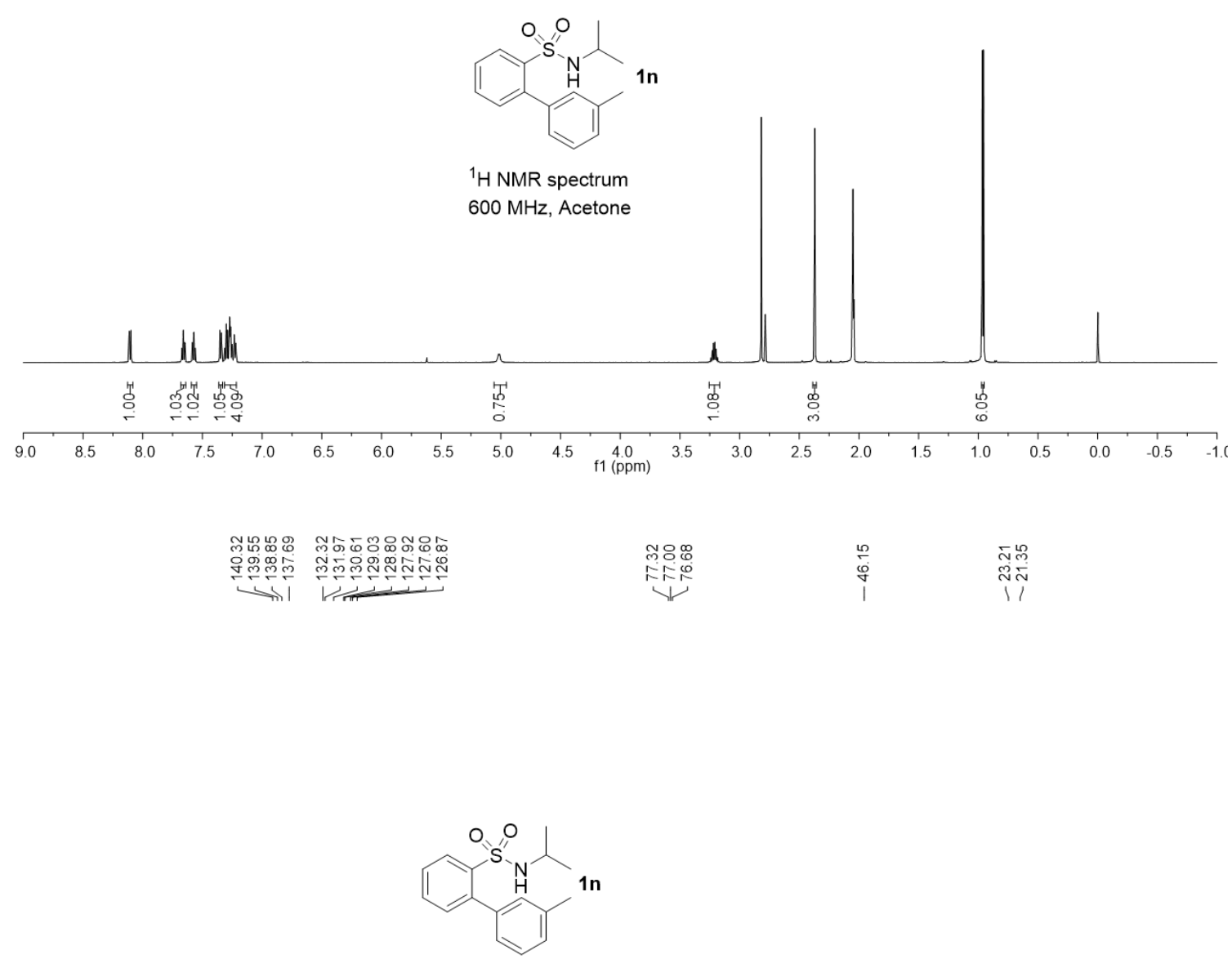

${ }^{13} \mathrm{C}$ NMR spectrum

$100 \mathrm{MHz}^{\mathrm{CDCl}_{3}}$

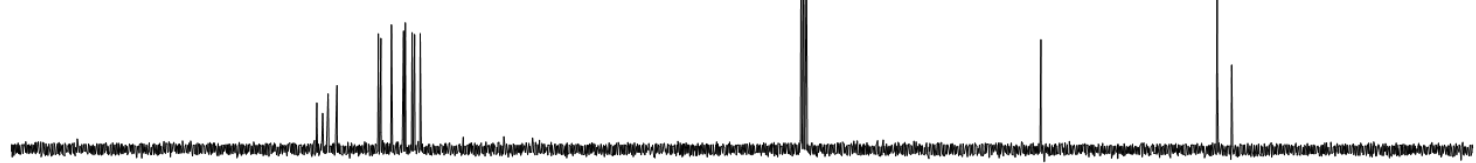

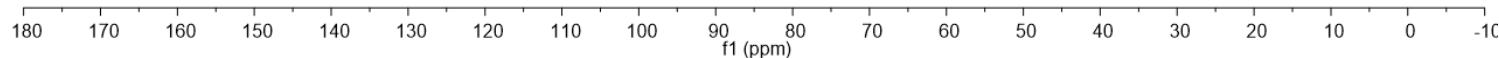



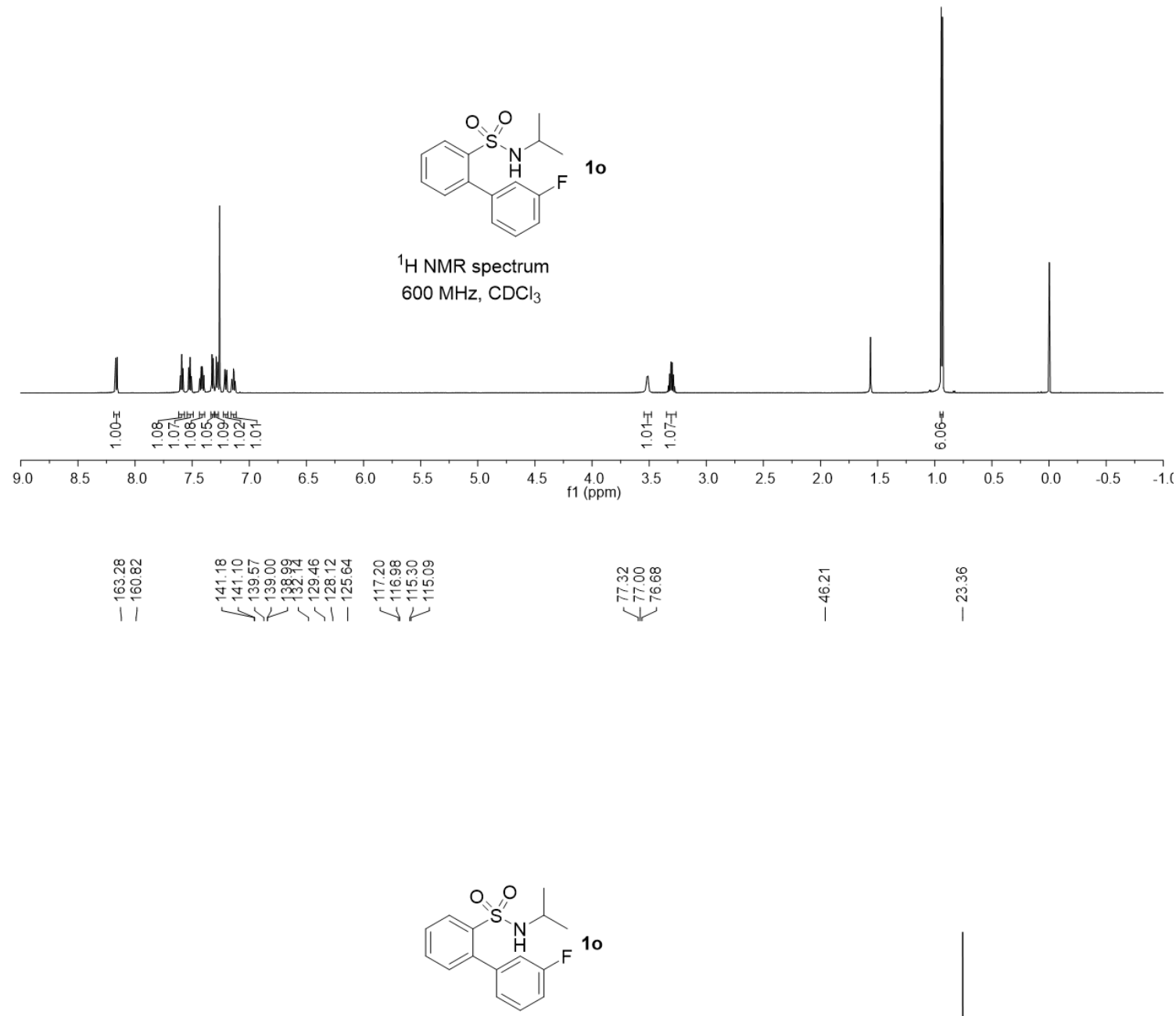

${ }^{13} \mathrm{C}$ NMR spectrum $100 \mathrm{MHz}, \mathrm{CDCl}_{3}$ 


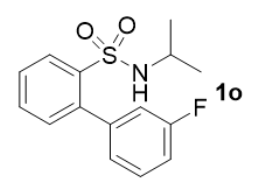

${ }^{19} \mathrm{~F}$ NMR spectrum

$375 \mathrm{MHz}, \mathrm{CDCl}_{3}$
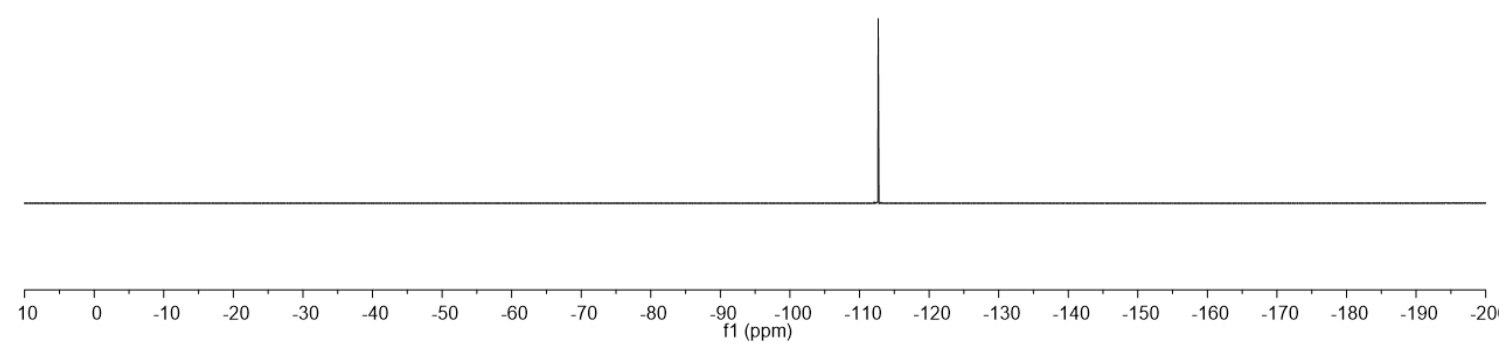

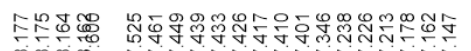

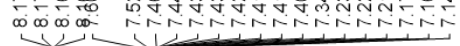

ل

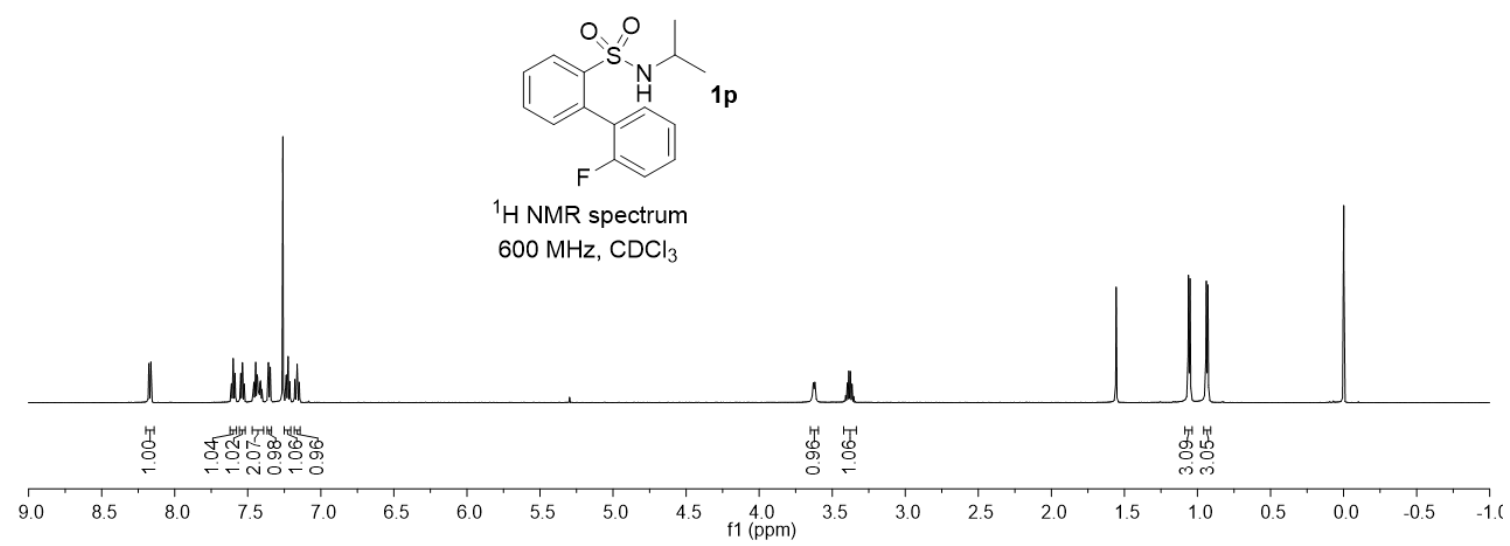



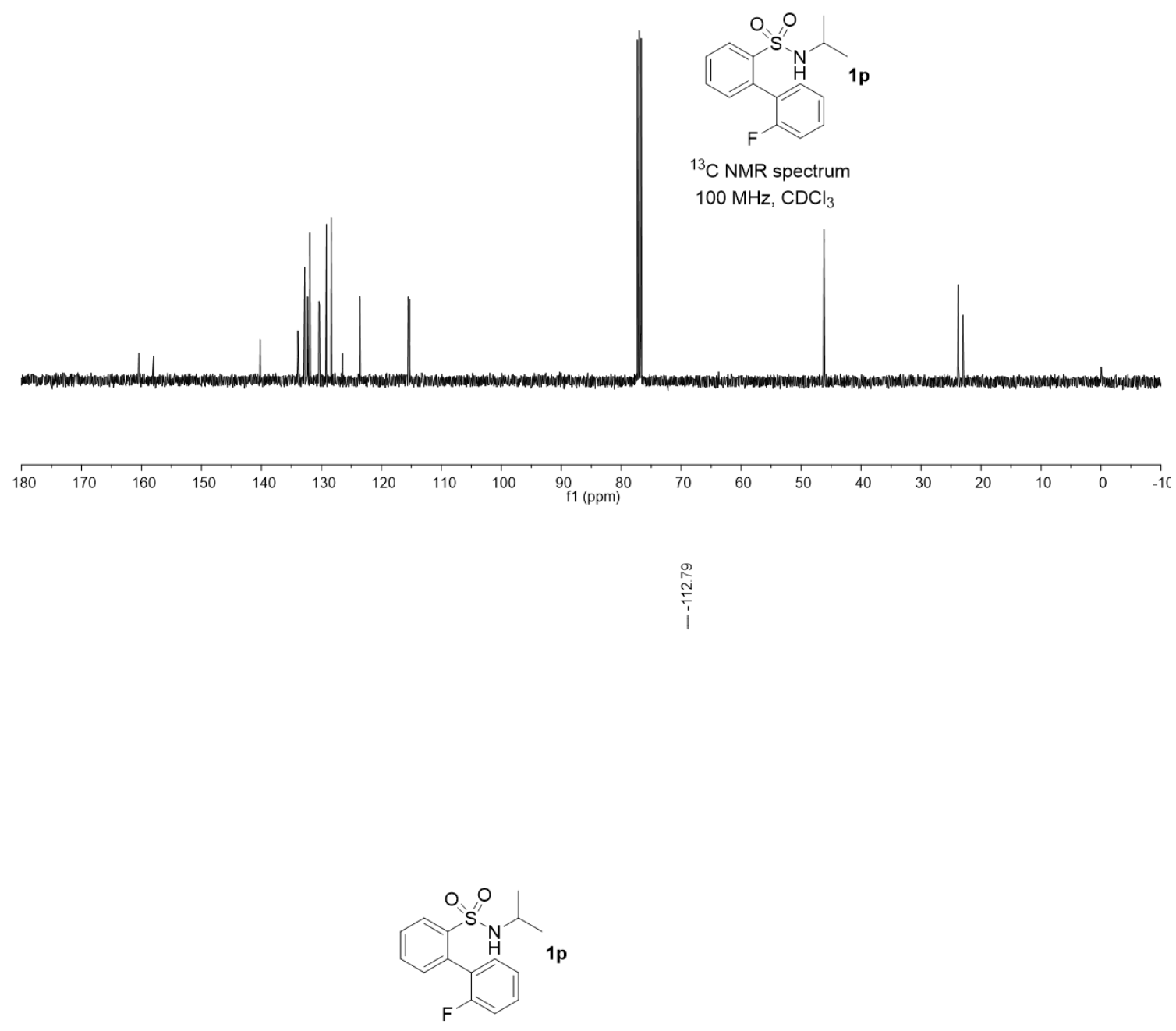

${ }^{19} \mathrm{~F}$ NMR spectrum

$375 \mathrm{MHz}, \mathrm{CDCl}_{3}$

\begin{tabular}{rllllllllllllllllllllll}
\hline & 10 & 0 & -10 & -20 & -30 & -40 & -50 & -60 & -70 & -80 & -90 & -100 & -110 & -120 & -130 & -140 & -150 & -160 & -170 & -180 & -190 & -20
\end{tabular} 


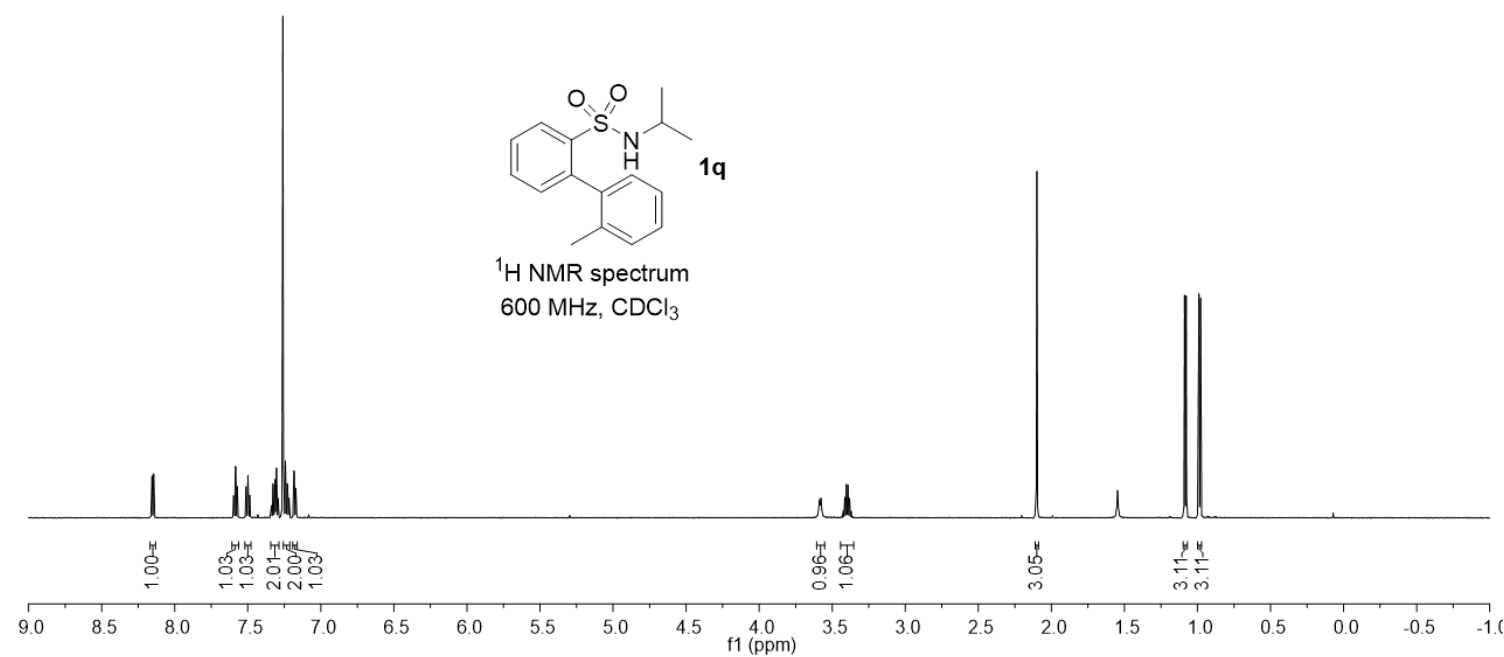

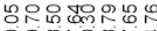

वृ.

पiि

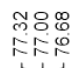

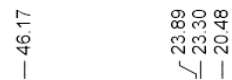

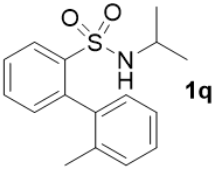

${ }^{13} \mathrm{C}$ NMR spectrum

$100 \mathrm{MHz} \mathrm{CDCl}_{3}$

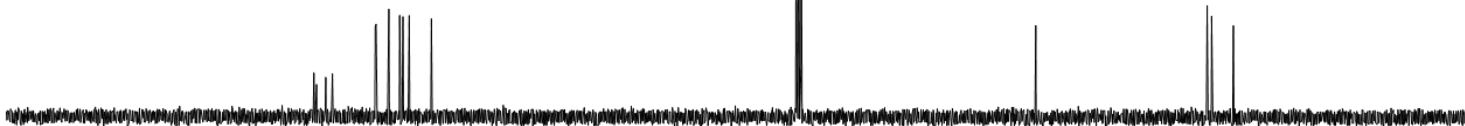

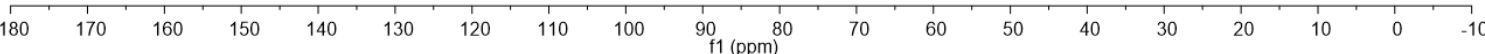




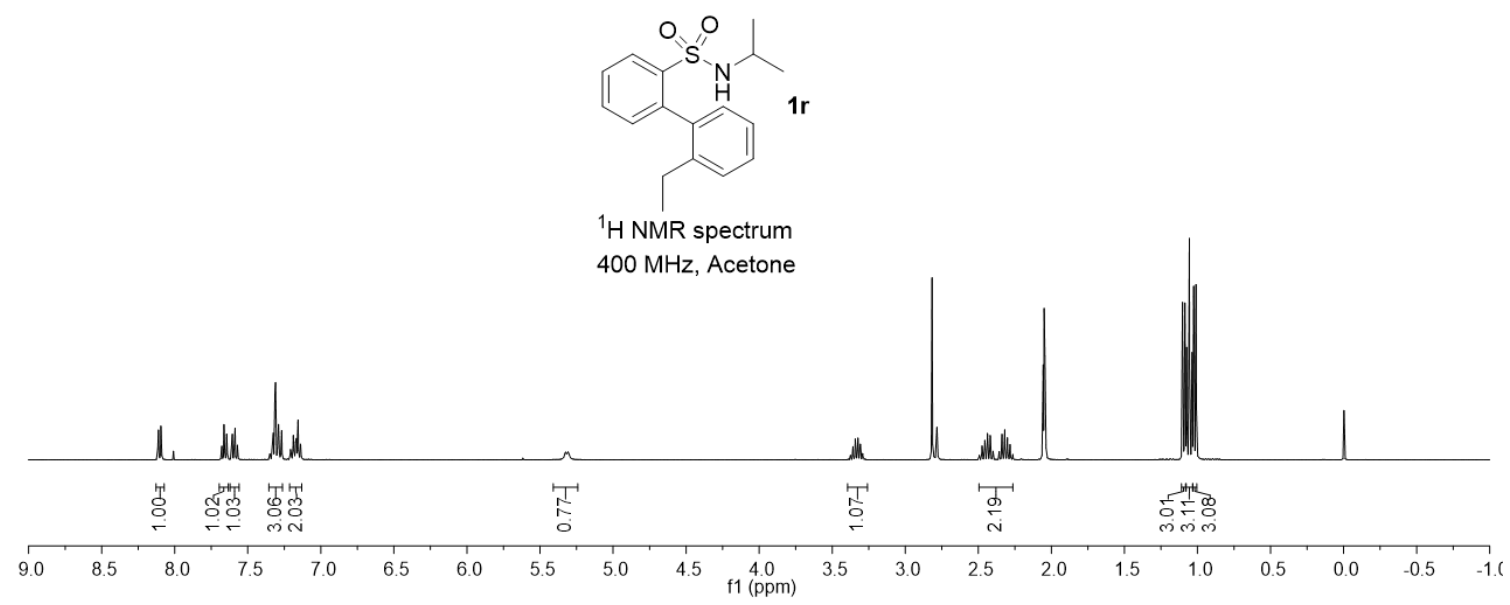

ฮิ

竞

i

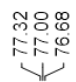

음

חiñ

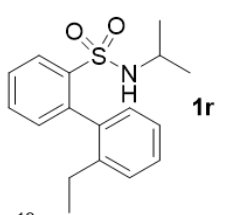

${ }^{13} \mathrm{C}$ NMR spectrum $100 \mathrm{MHz}, \mathrm{CDCl}_{3}$

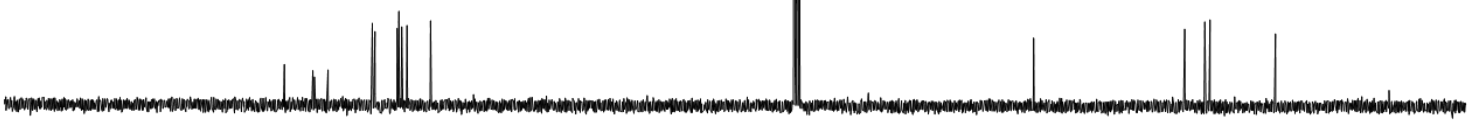

\begin{tabular}{rlllllllllllllllllllllll}
\hline 180 & 170 & 160 & 150 & 140 & 130 & 120 & 110 & 100 & 90 & 80 & 70 & 60 & 50 & 40 & 30 & 20 & 10 & 0 & -10
\end{tabular} 


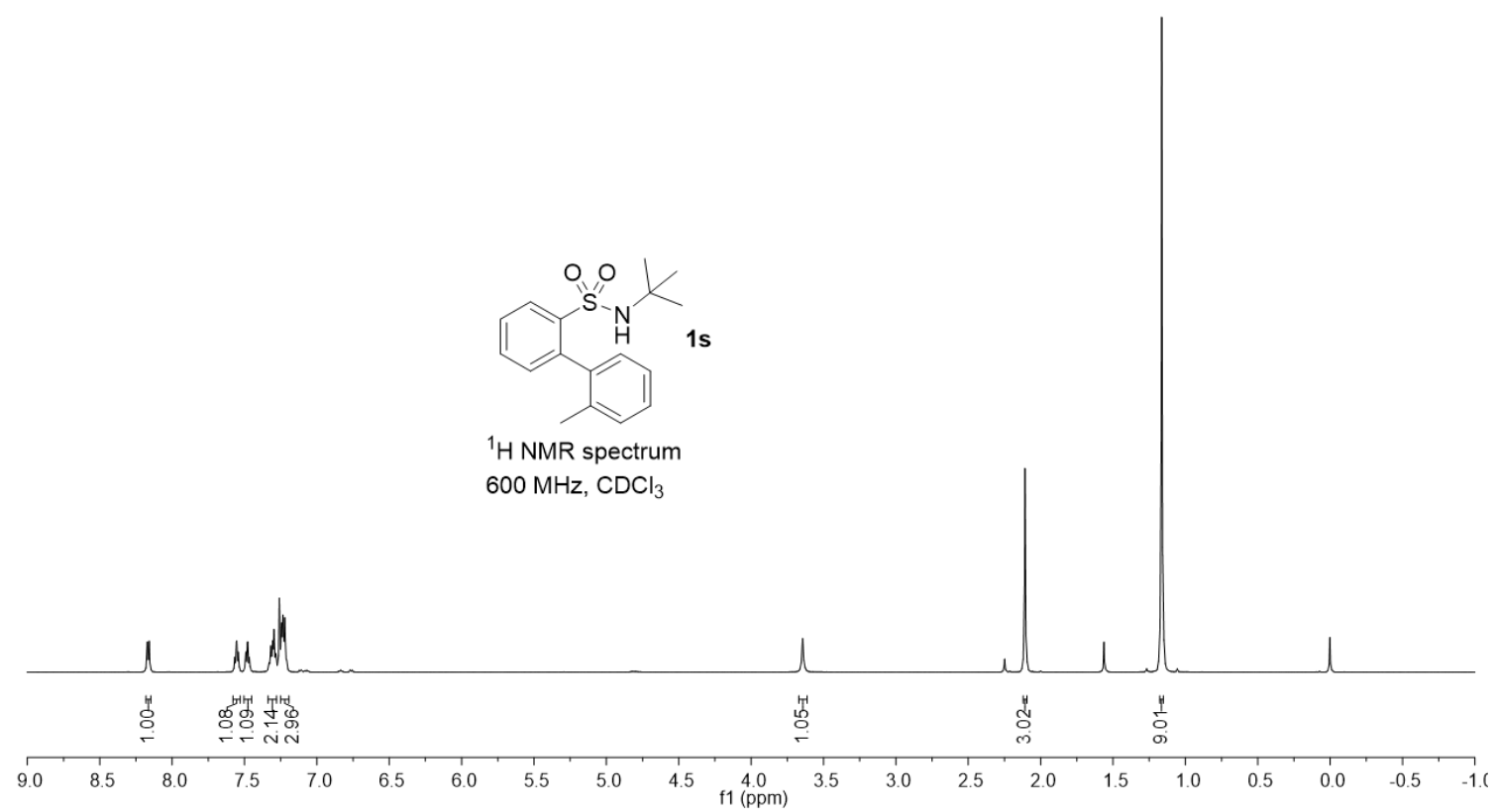

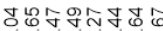

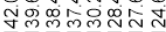

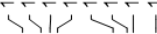

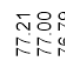

$\begin{array}{lll}0 & 0 \\ 0 & 0 & 0 \\ 0 & 0 & 0 \\ 0 & 0 & 1\end{array}$

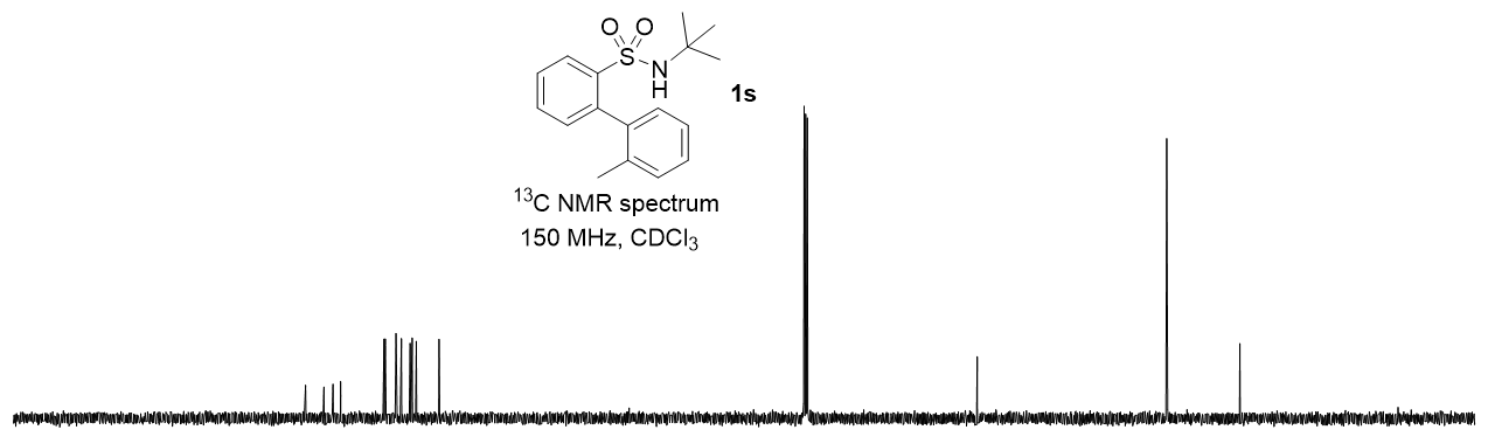

$\begin{array}{llllllllllllllllllll}180 & 170 & 160 & 150 & 140 & 130 & 120 & 110 & 100 & 90 & 80 & 70 & 60 & 50 & 40 & 30 & 20 & 10 & 0 & -10\end{array}$ 


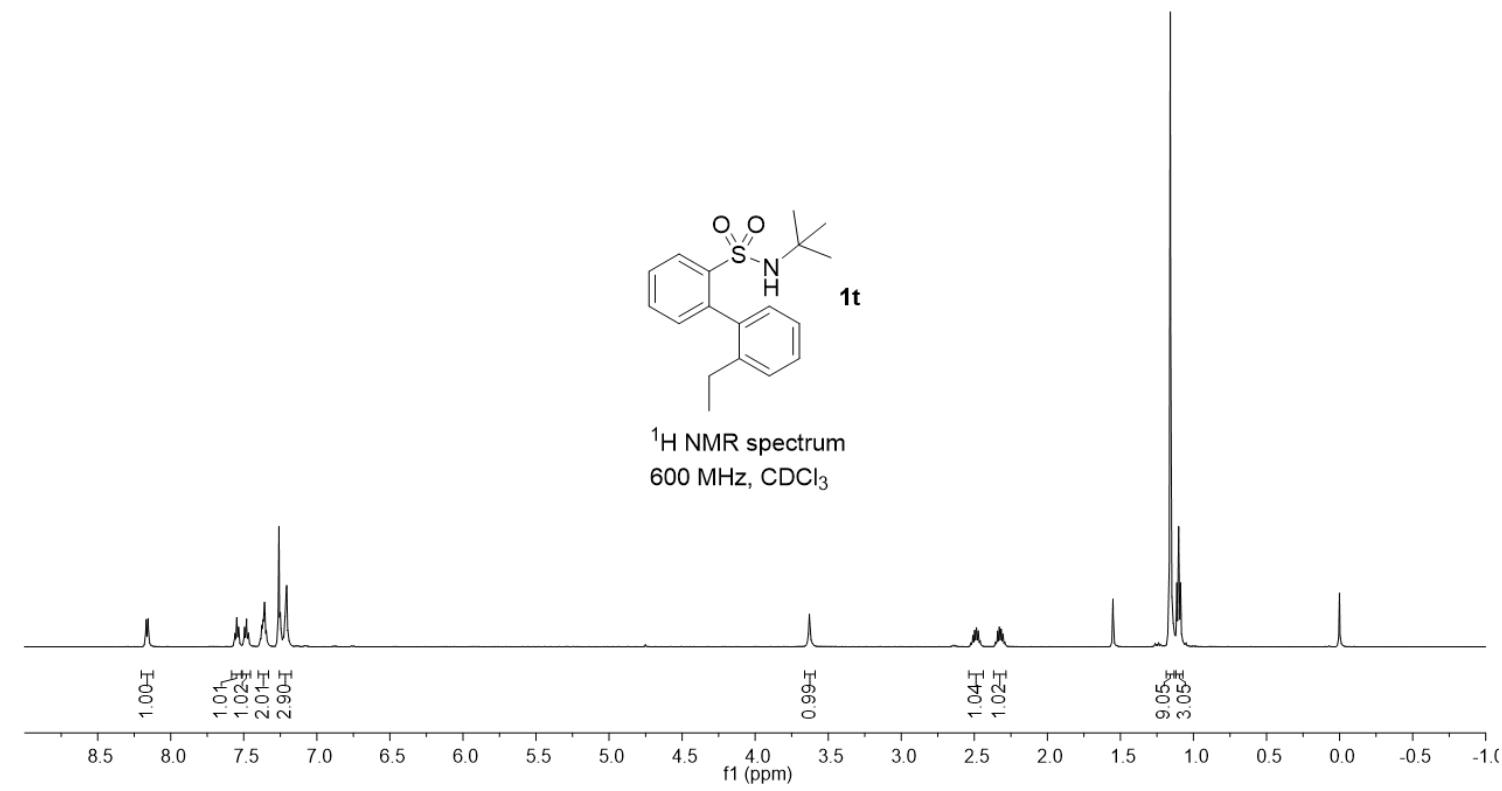

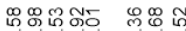

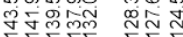

पiा

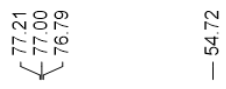

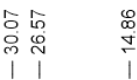

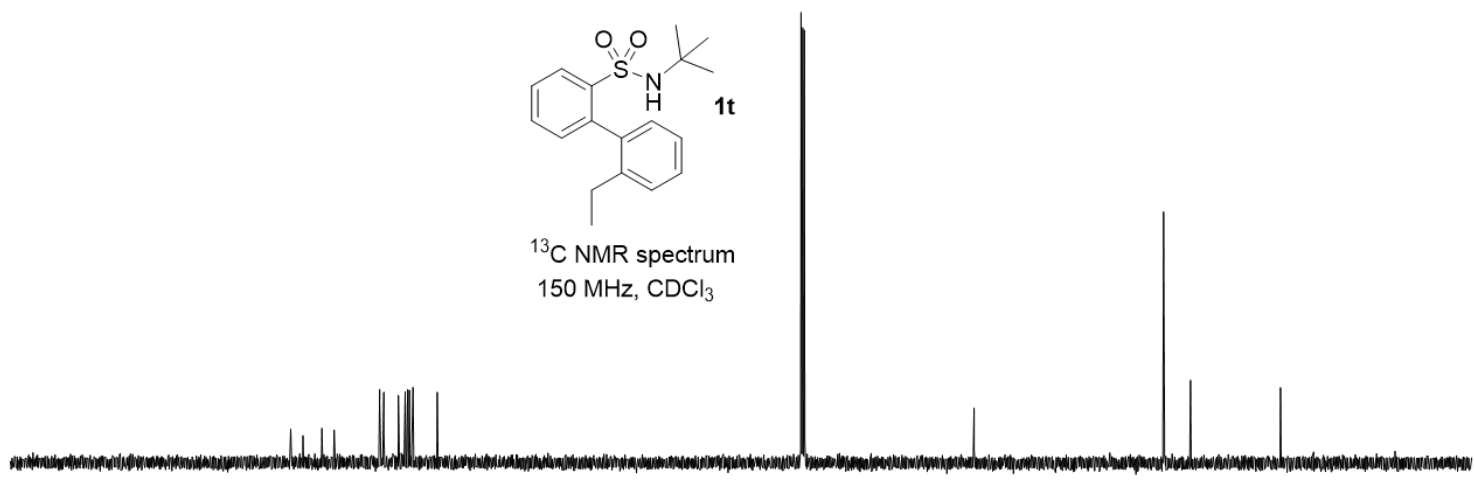

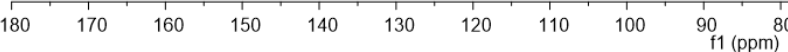




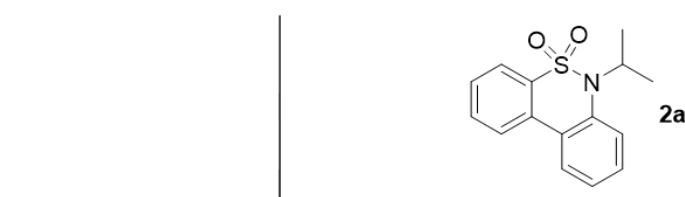

${ }^{1} \mathrm{H}$ NMR spectrum

$600 \mathrm{MHz}, \mathrm{CDCl}_{3}$

ए

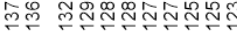

। 115

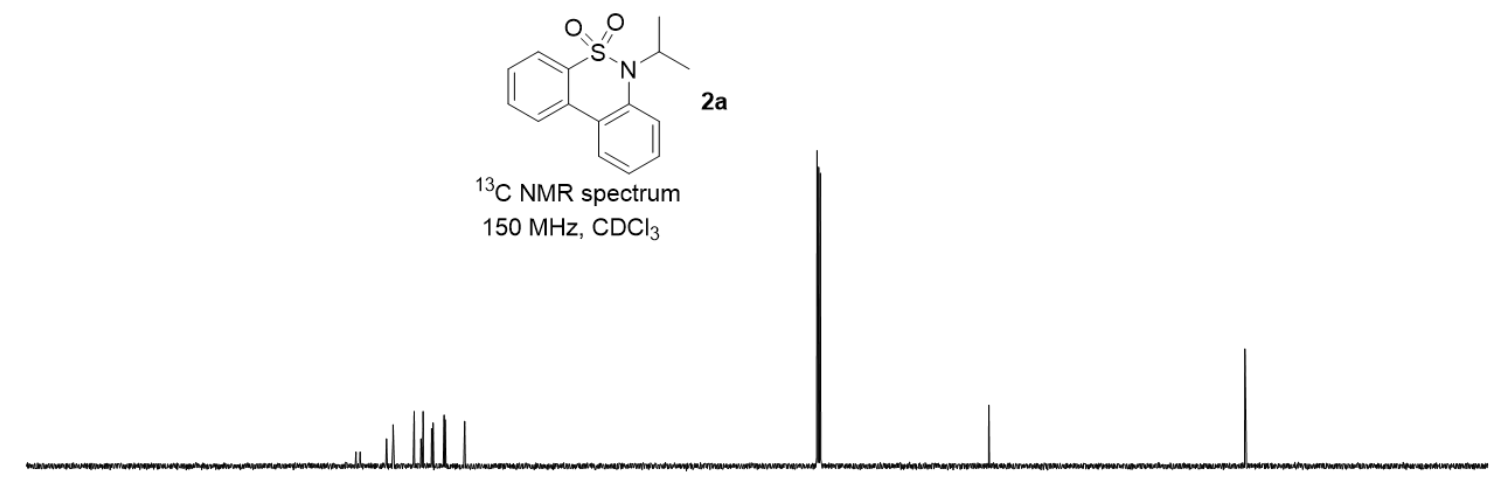

$\begin{array}{llllllllllllllllllll}180 & 170 & 160 & 150 & 140 & 130 & 120 & 110 & 100 & 90 & 80 & 70 & 60 & 50 & 40 & 30 & 20 & 10 & 0 & -10\end{array}$ 


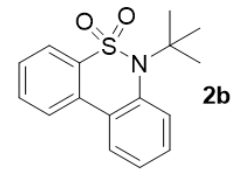

${ }^{1} \mathrm{H}$ NMR spectrum $600 \mathrm{MHz}, \mathrm{CDCl}_{3}$

每

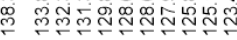

1 it

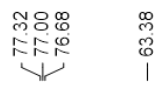

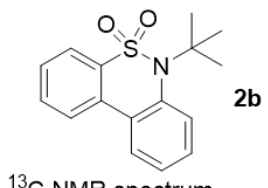

${ }^{13} \mathrm{C}$ NMR spectrum

$100 \mathrm{MHz}, \mathrm{CDCl}_{3}$

2b

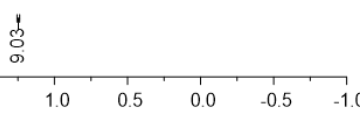




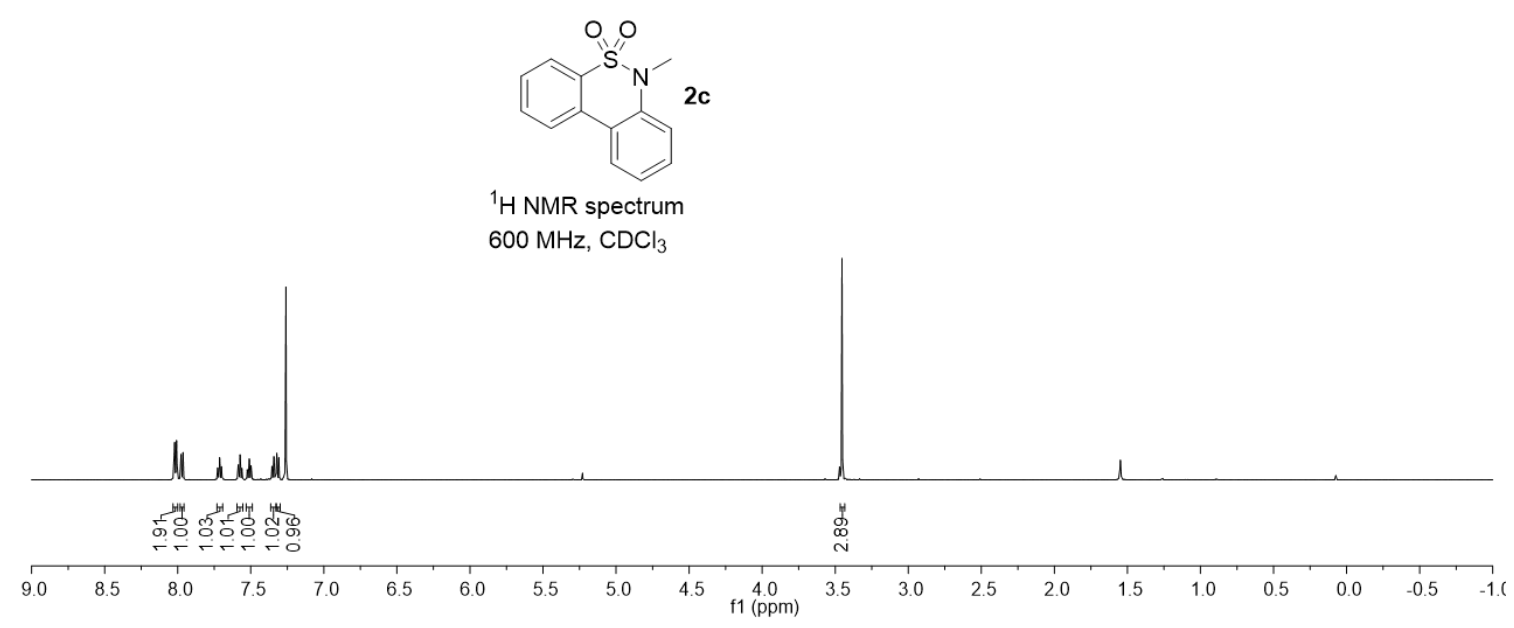

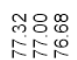

in

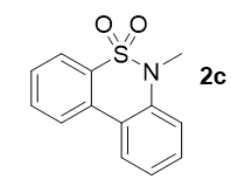

${ }^{13} \mathrm{C}$ NMR spectrum

$100 \mathrm{MHz} \mathrm{CDCl}_{3}$

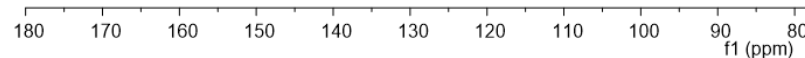




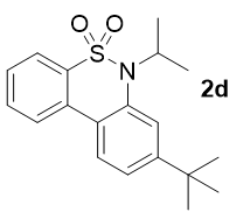

${ }^{1} \mathrm{H}$ NMR spectrum $600 \mathrm{MHz}, \mathrm{CDCl}_{3}$
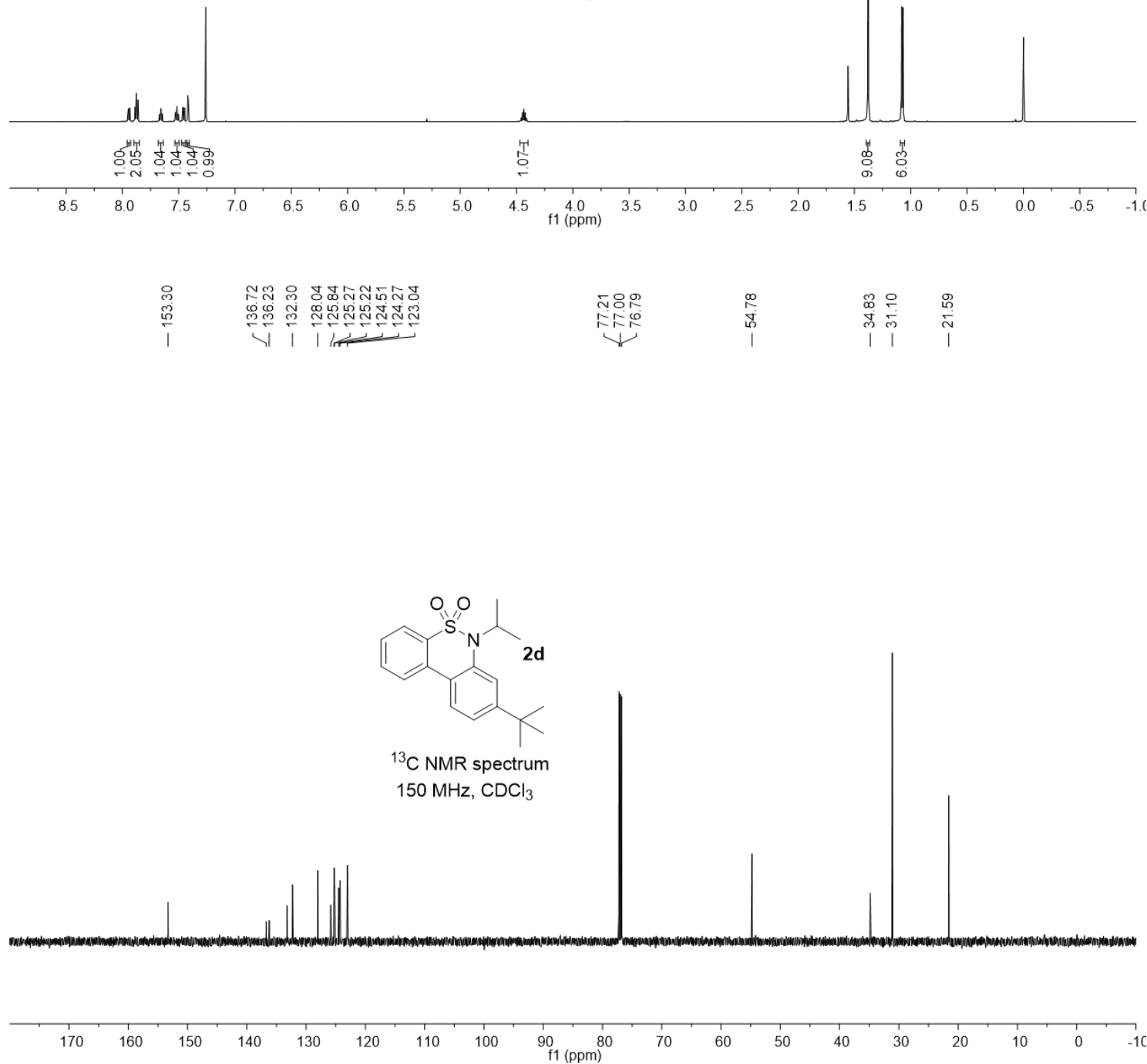


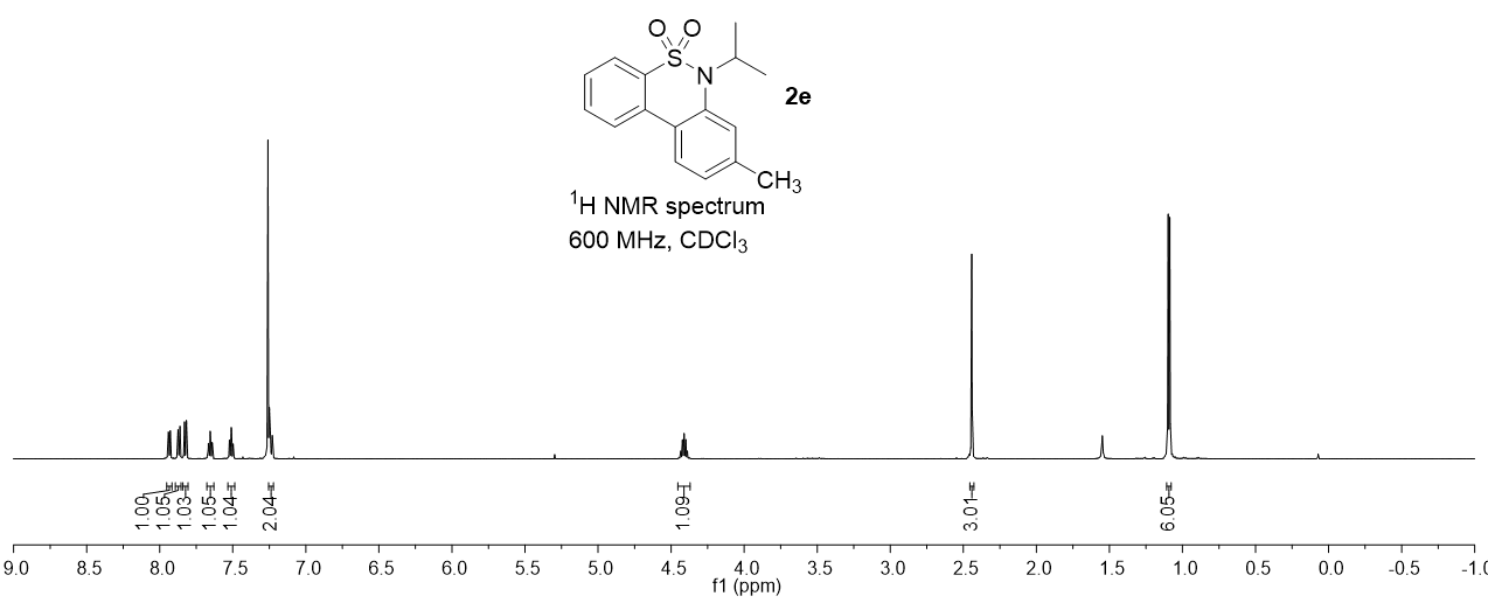

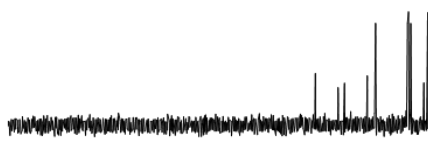

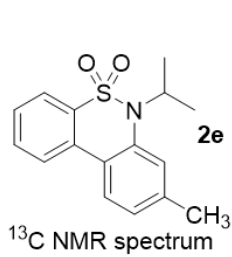
$100 \mathrm{MHz}, \mathrm{CDCl}_{3}$

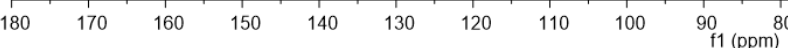



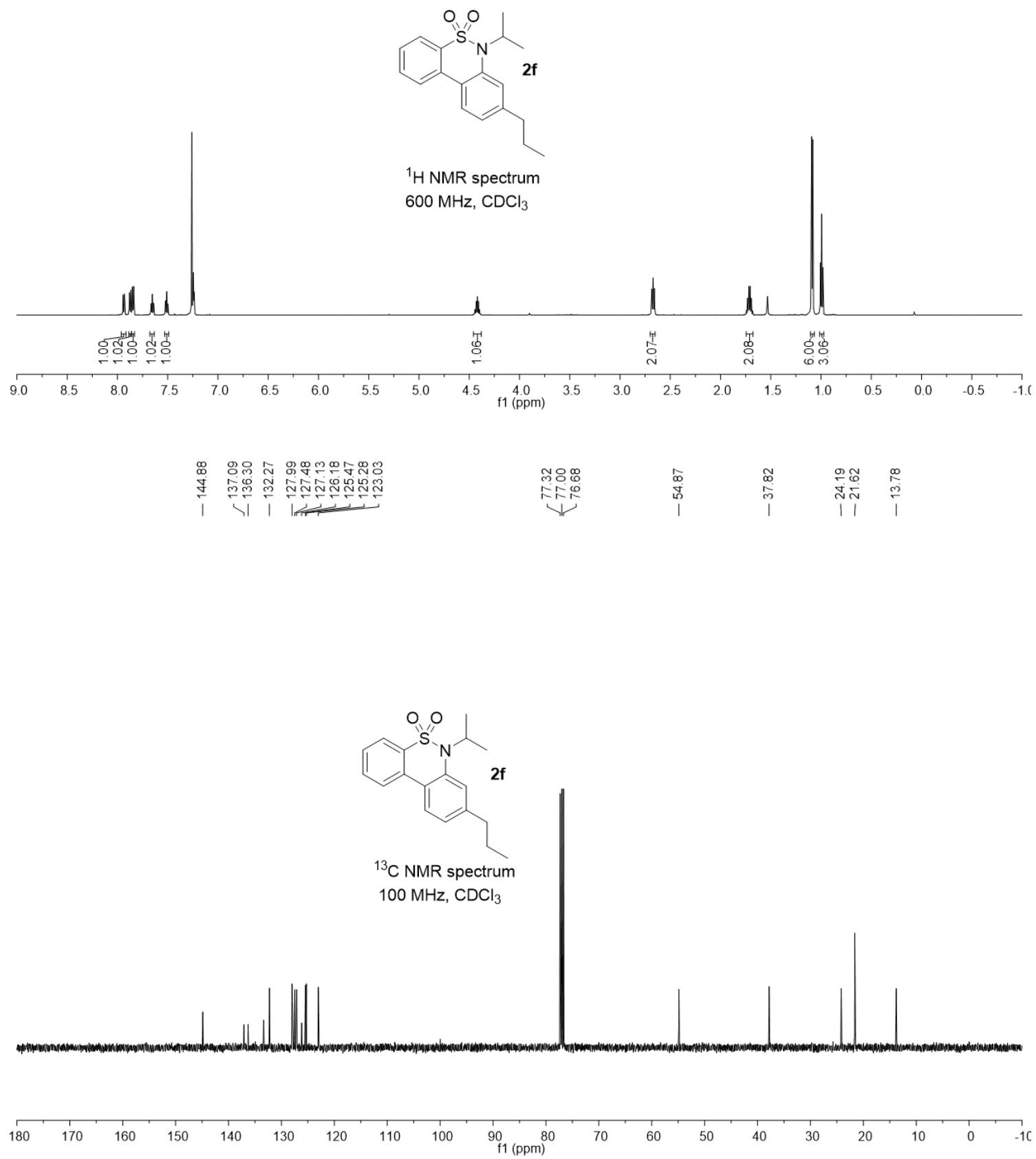



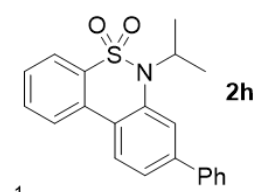

${ }^{1} \mathrm{H}$ NMR spectrum $600 \mathrm{MHz}, \mathrm{CDCl}_{3}$
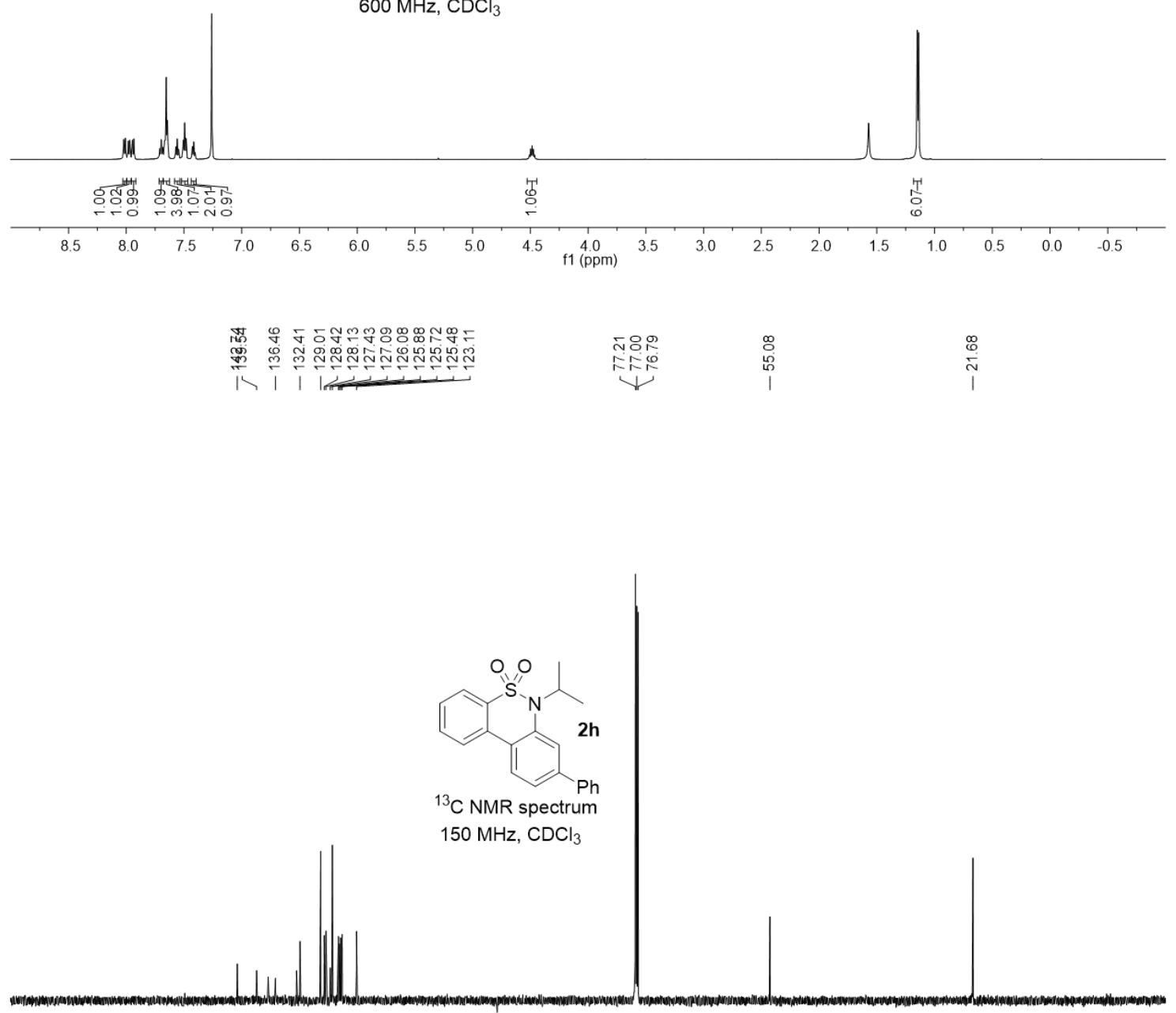

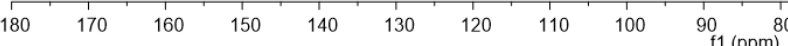




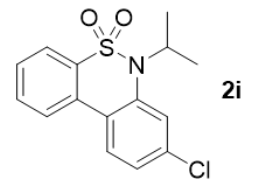

${ }^{1} \mathrm{H}$ NMR spectrum $400 \mathrm{MHz}, \mathrm{CDCl}_{3}$
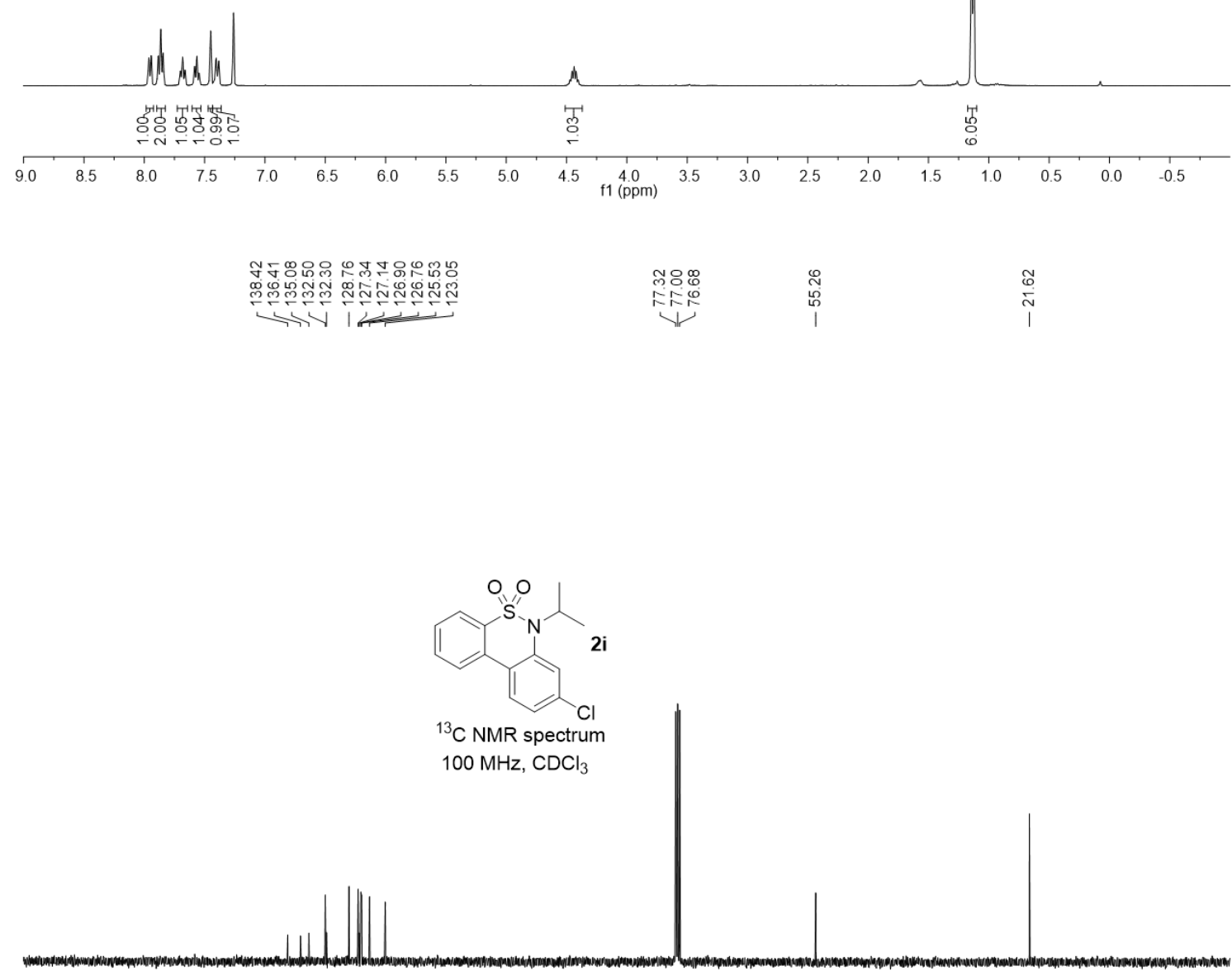

180

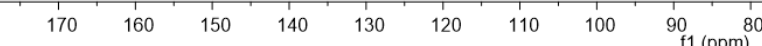




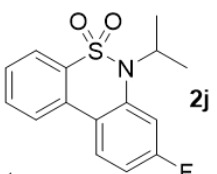

${ }^{1} \mathrm{H}$ NMR spectrum

$400 \mathrm{MHz}, \mathrm{CDCl}_{3}$
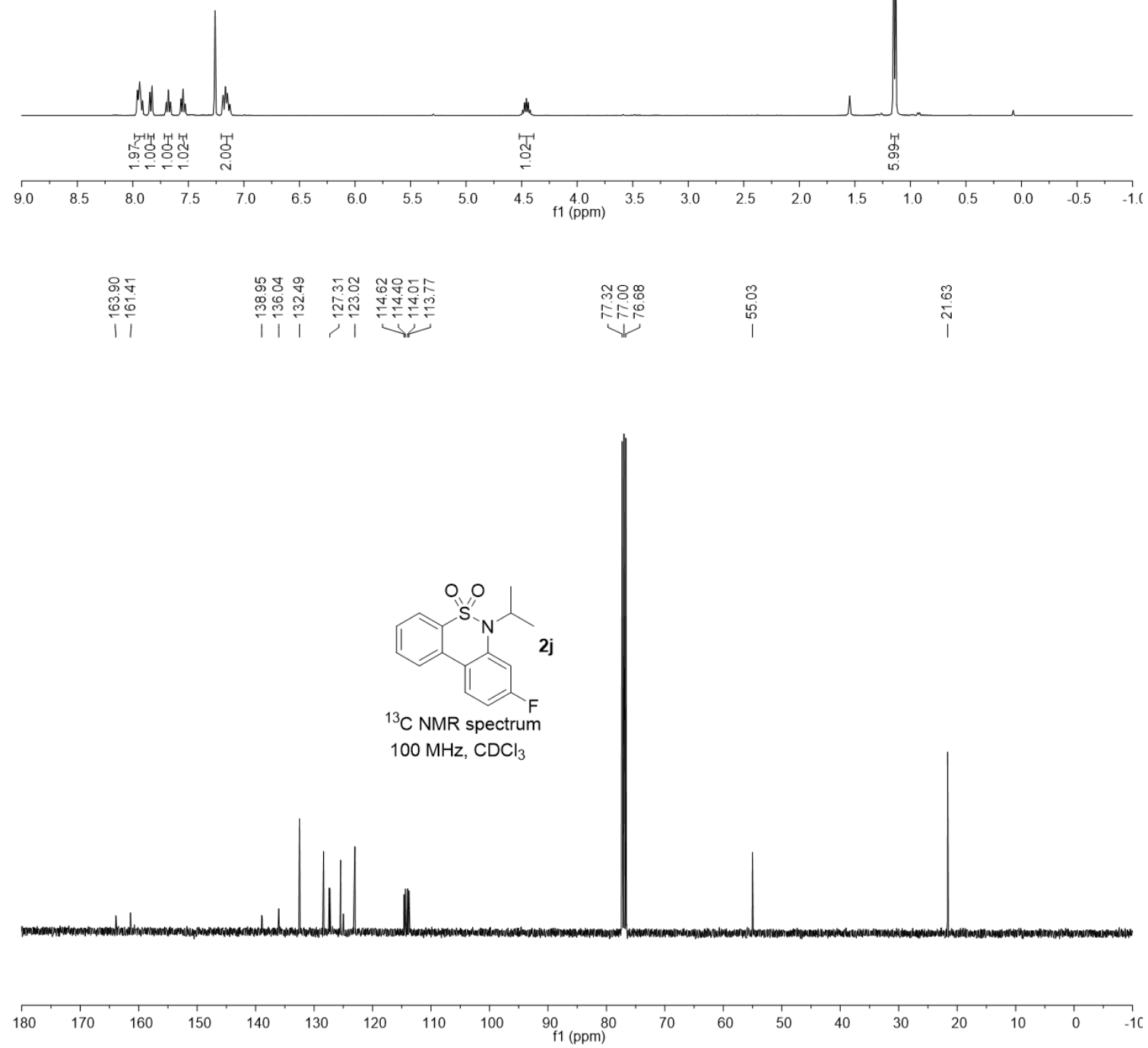


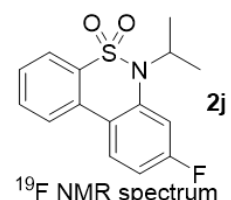

$375 \mathrm{MHz}, \mathrm{CDCl}_{3}$

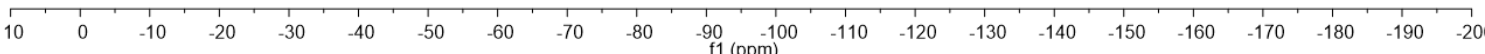

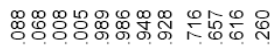

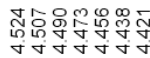

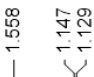

$\underbrace{\infty}$

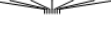

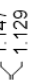

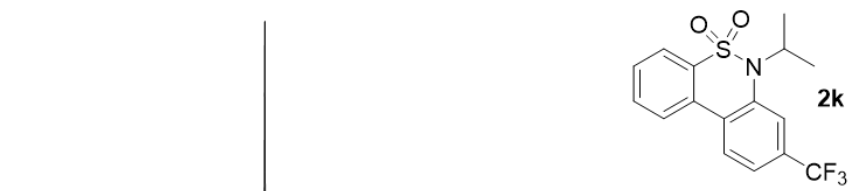

${ }^{1} \mathrm{H}$ NMR spectrum

$400 \mathrm{MHz}, \mathrm{CDCl}_{3}$

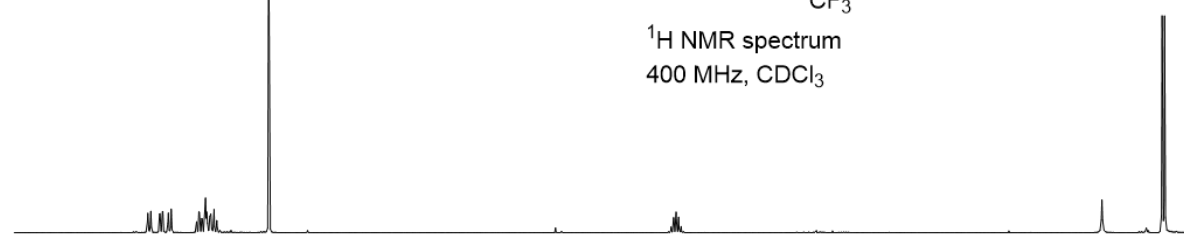

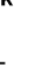

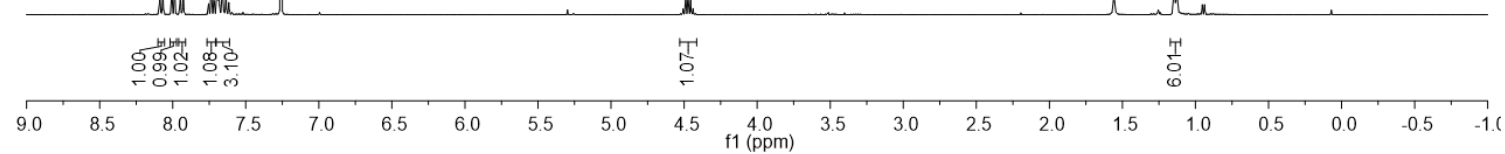



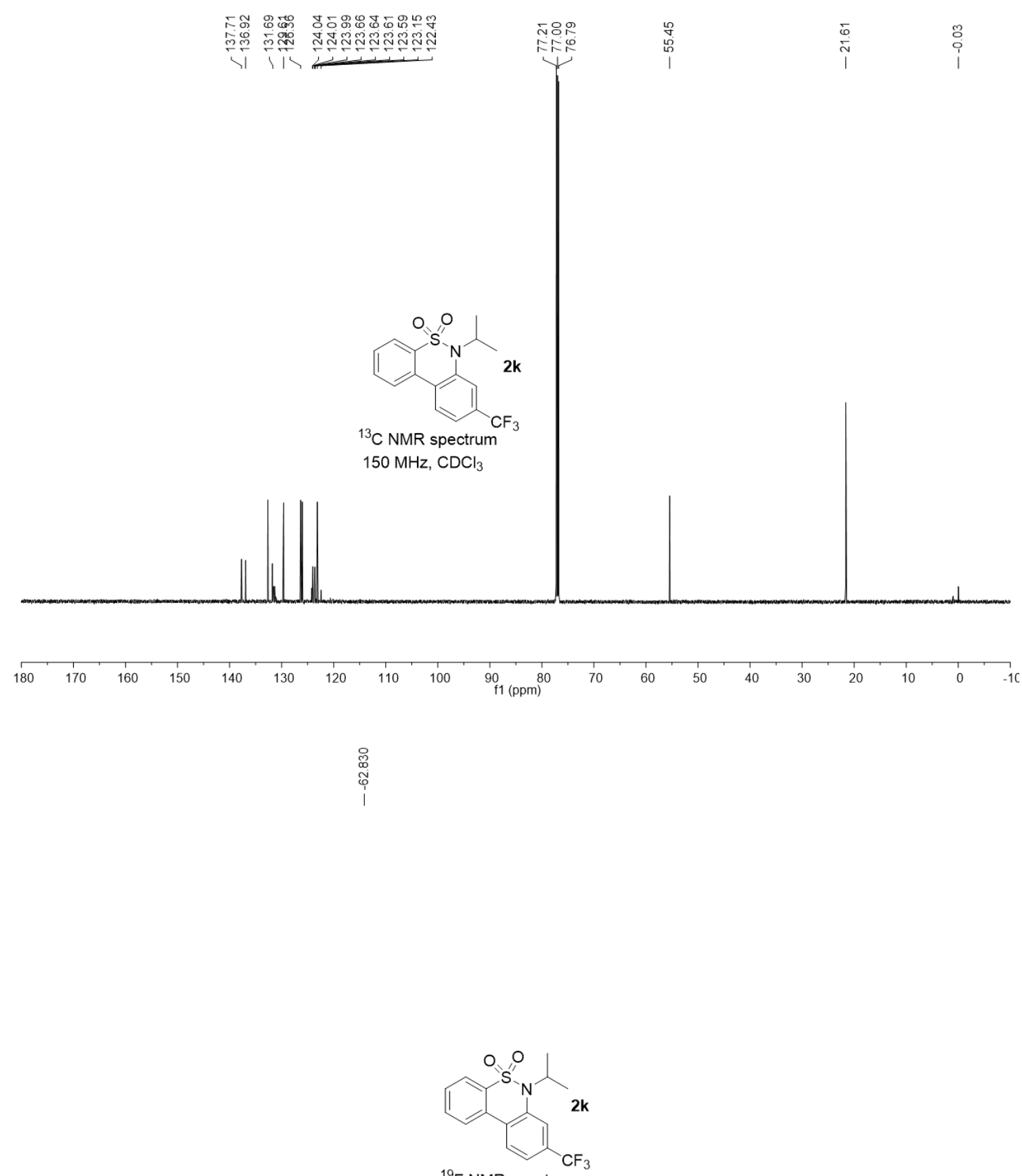

${ }^{19} \mathrm{~F}$ NMR spectrum

$375 \mathrm{MHz}^{\mathrm{CDCl}} \mathrm{CD}_{3}$

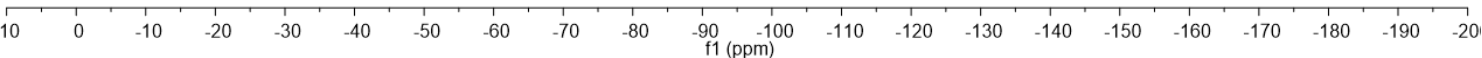




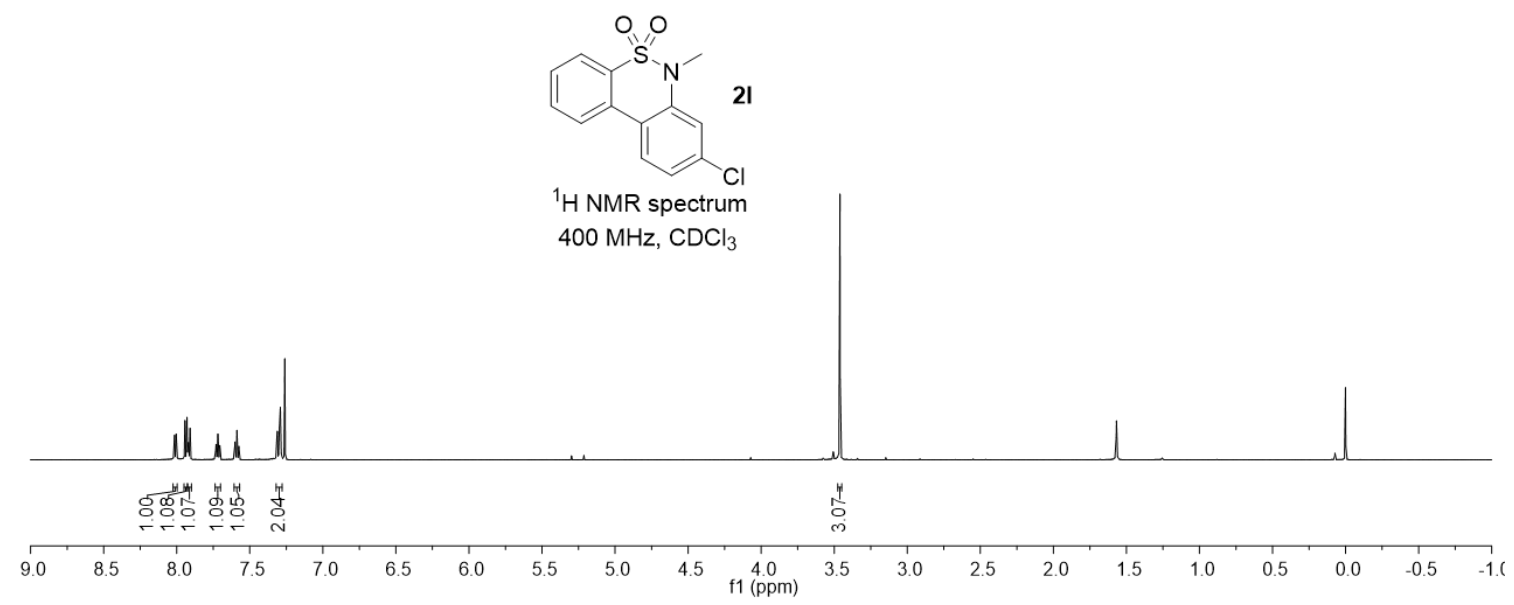

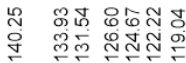

i

ل

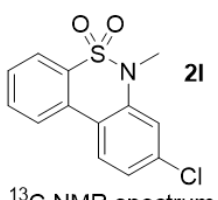

${ }^{13} \mathrm{C}$ NMR spectrum

$100 \mathrm{MHz} \mathrm{CDCl}_{3}$

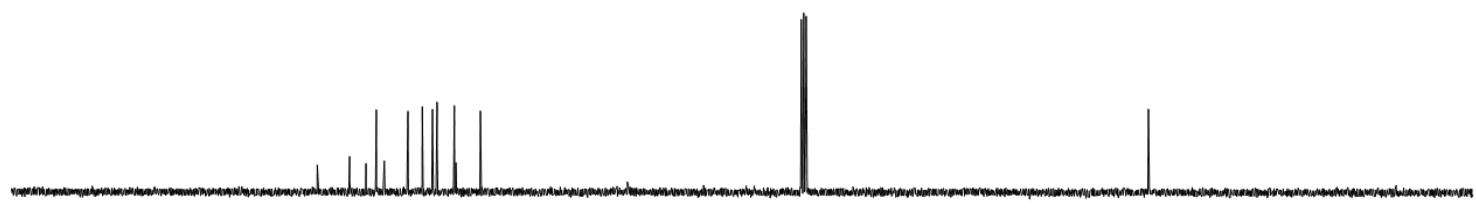

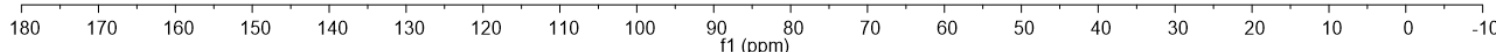




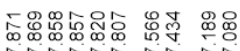

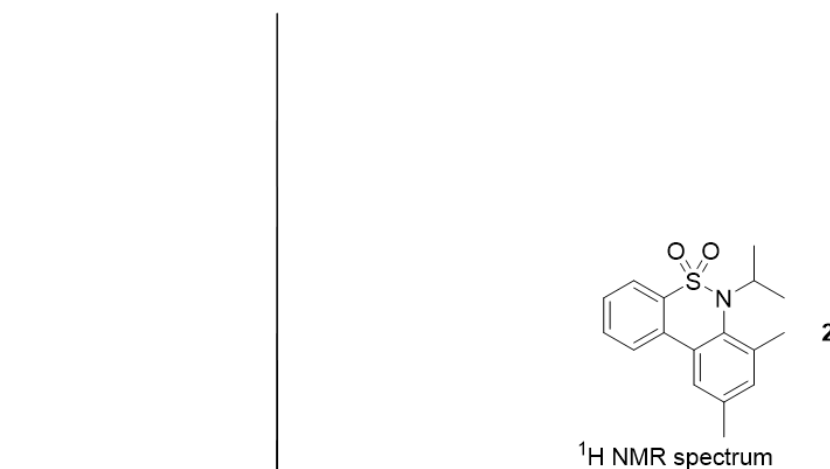

$2 m$

$600 \mathrm{MHz}, \mathrm{CDCl}_{3}$
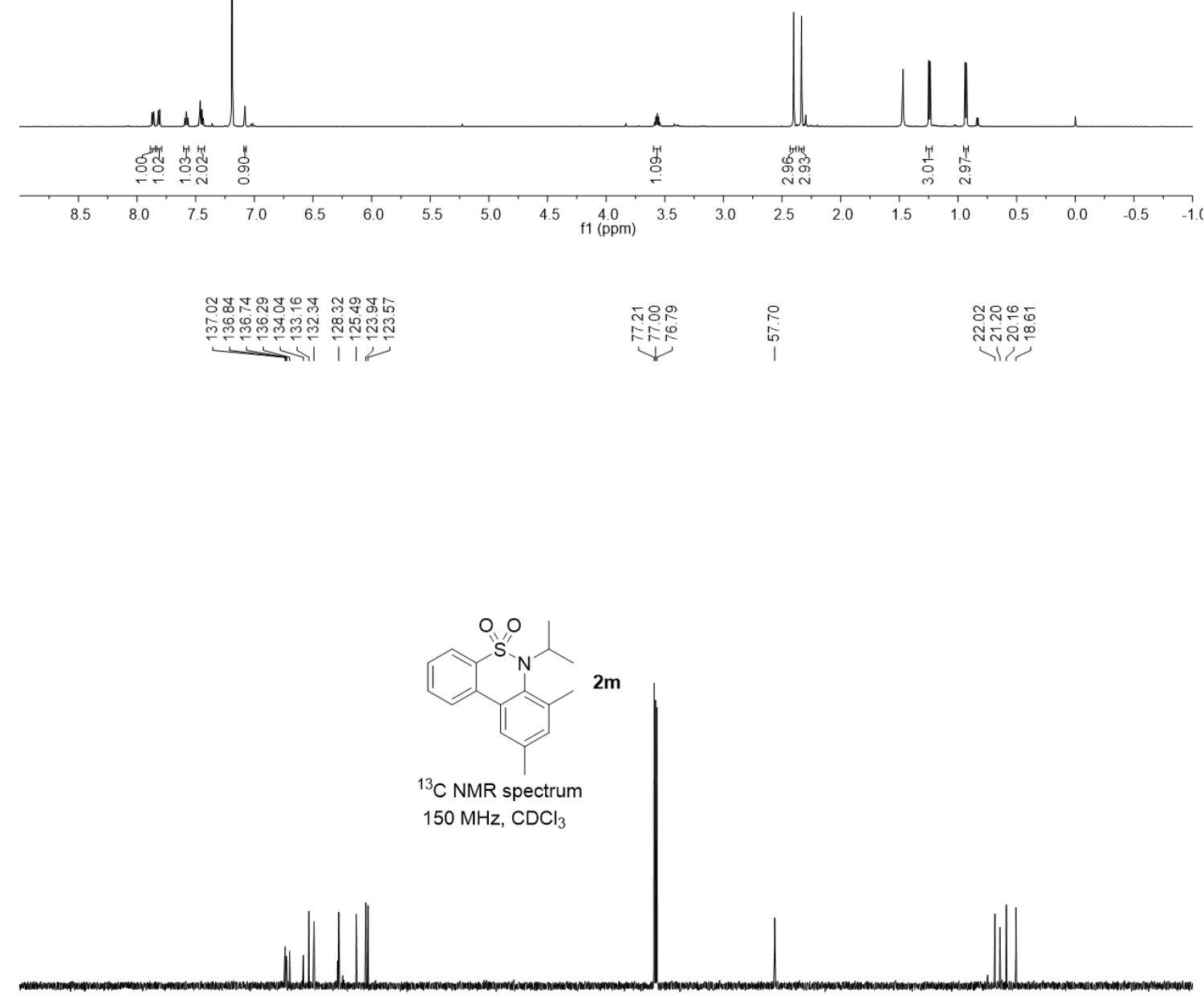

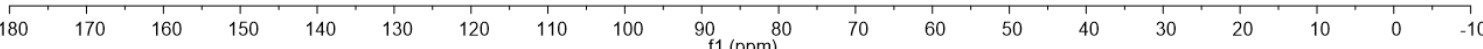




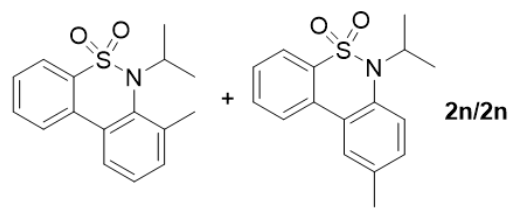

${ }^{1} \mathrm{H}$ NMR spectrum $400 \mathrm{MHz}$, Acetone
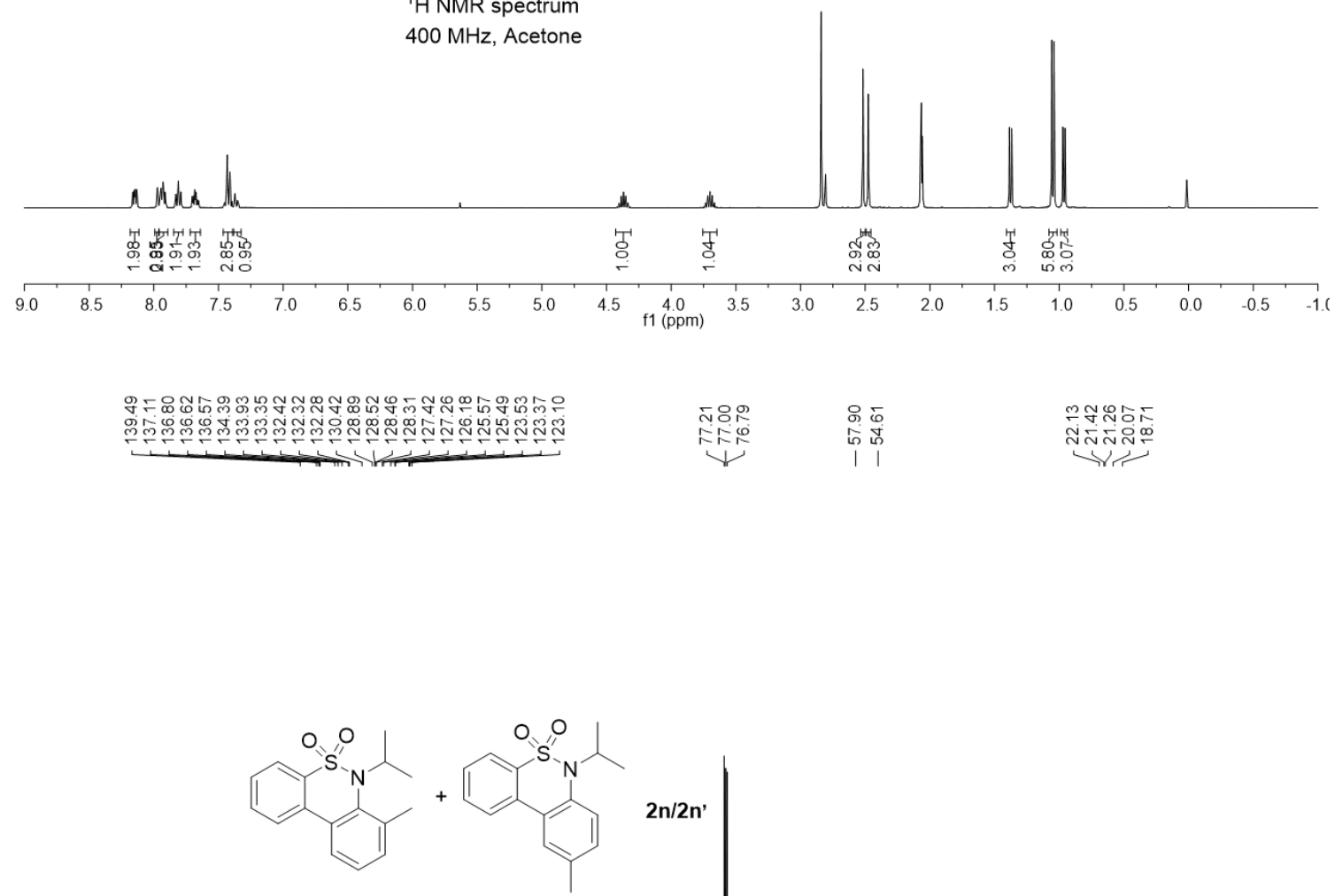

$2 n / 2 n$

${ }^{13} \mathrm{C}$ NMR spectrum

$150 \mathrm{MHz}, \mathrm{CDCl}_{3}$

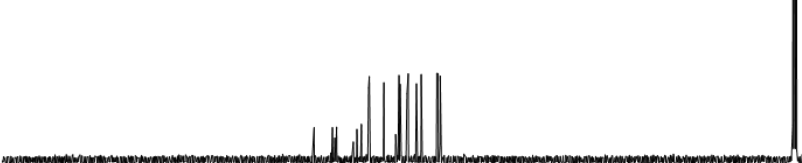

\begin{tabular}{llllllllll}
180 & 170 & 160 & 150 & 140 & 130 & 120 & 110 & 100 & 90 \\
\hline $\mathrm{f} 1(\mathrm{ppm})$ & 1 & 1 & 1
\end{tabular} 

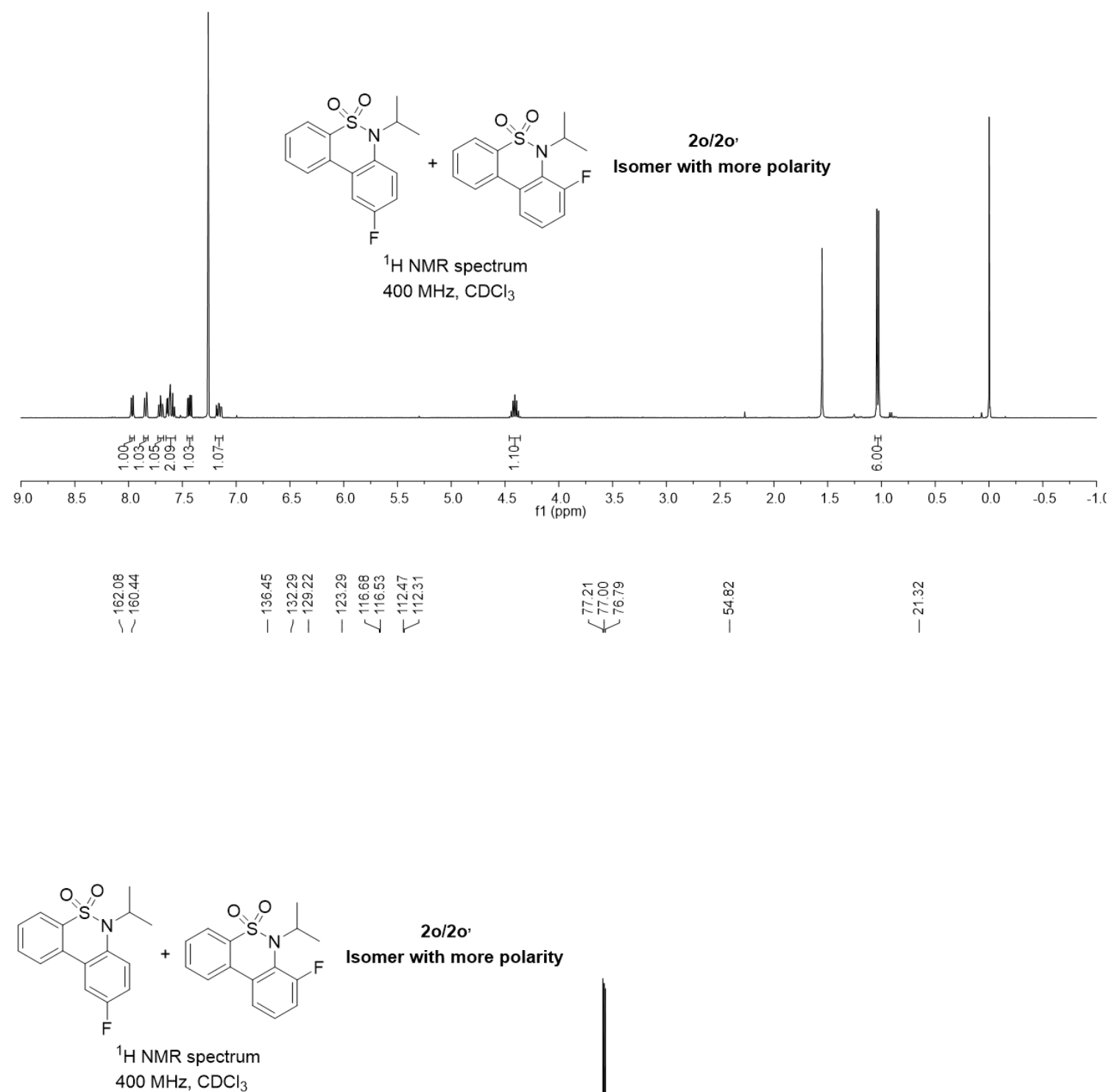

2o/2o'

Isomer with more polarity $400 \mathrm{MHz}, \mathrm{CDCl}_{3}$

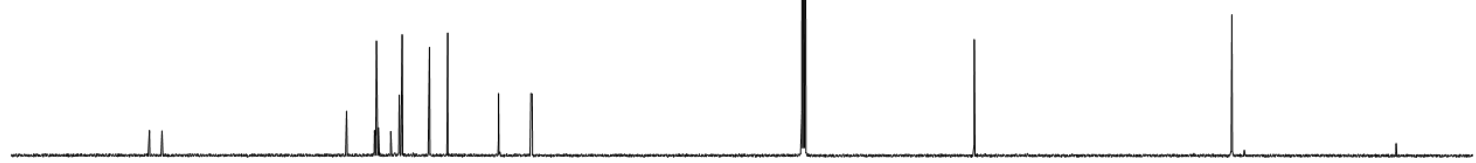

180

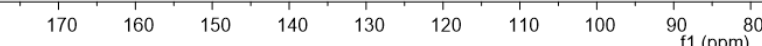
${ }^{80}$ 

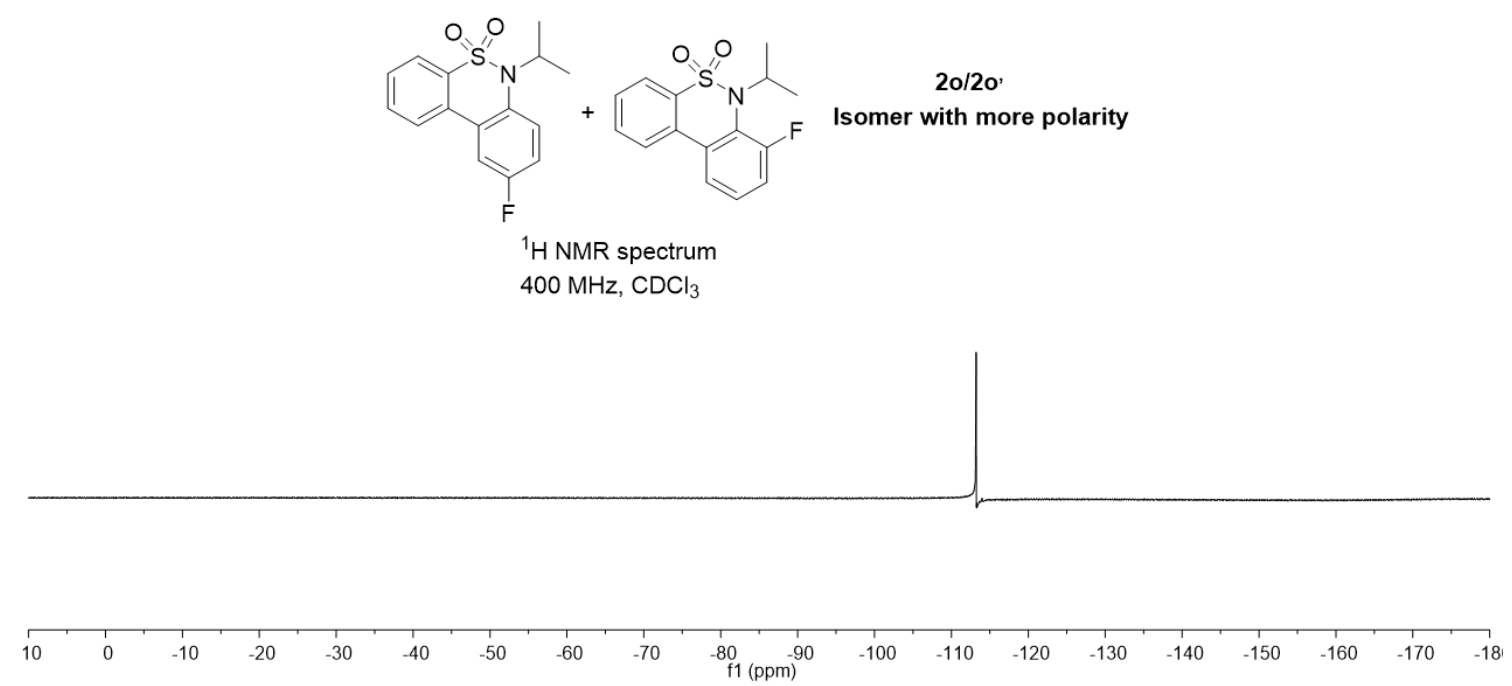

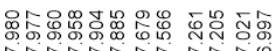

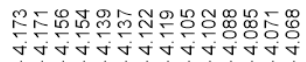

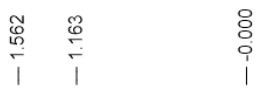

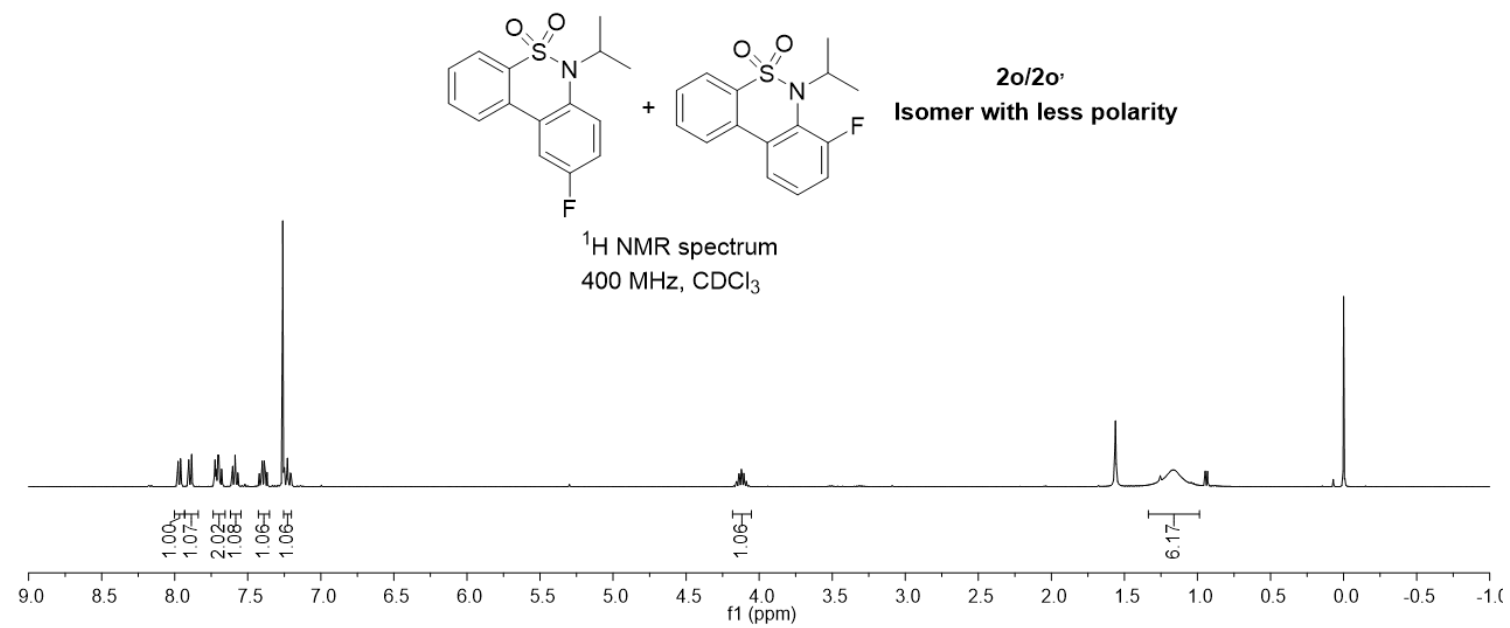




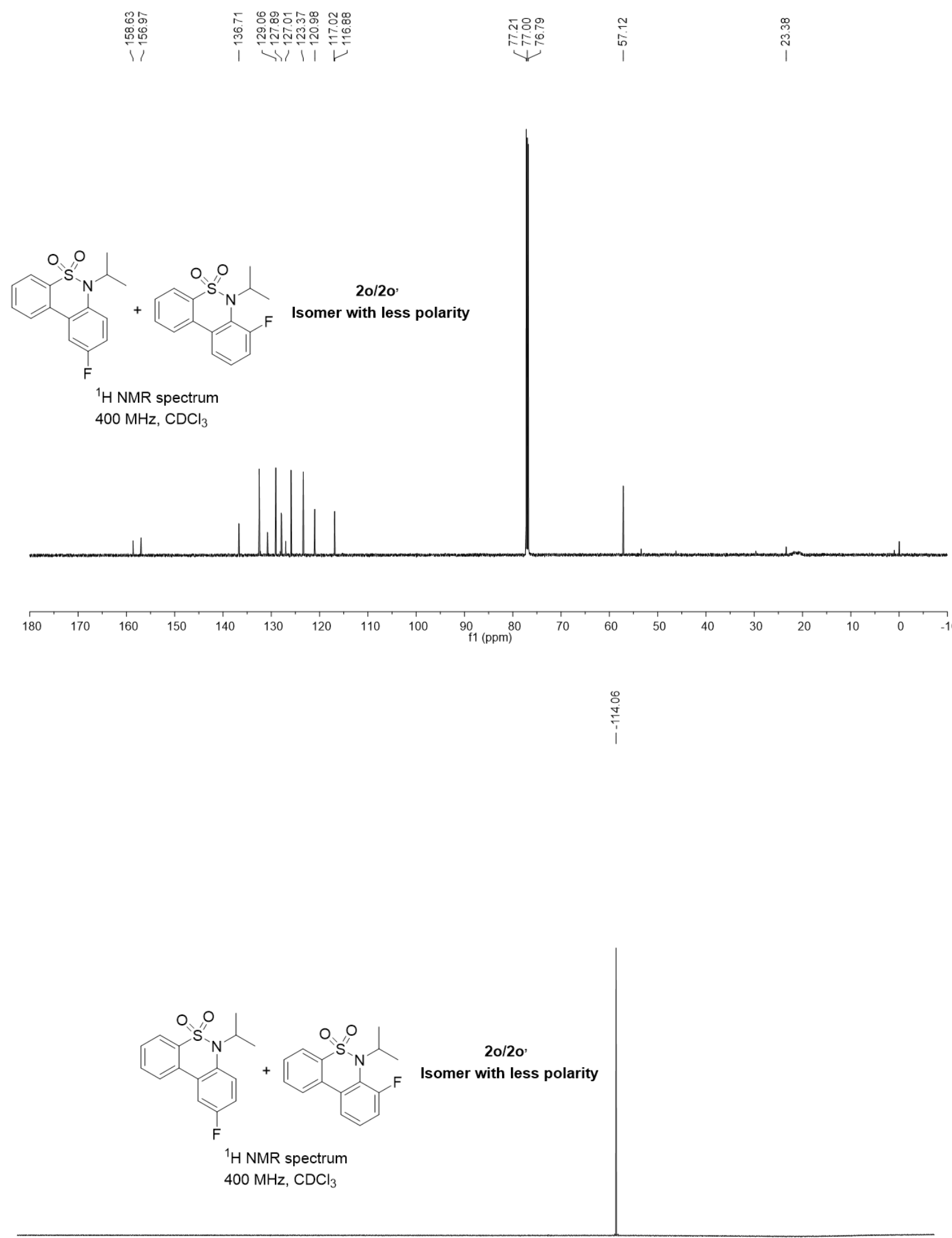

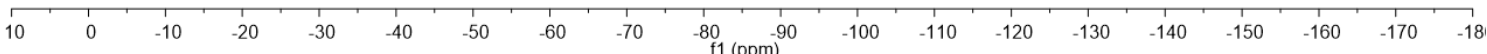



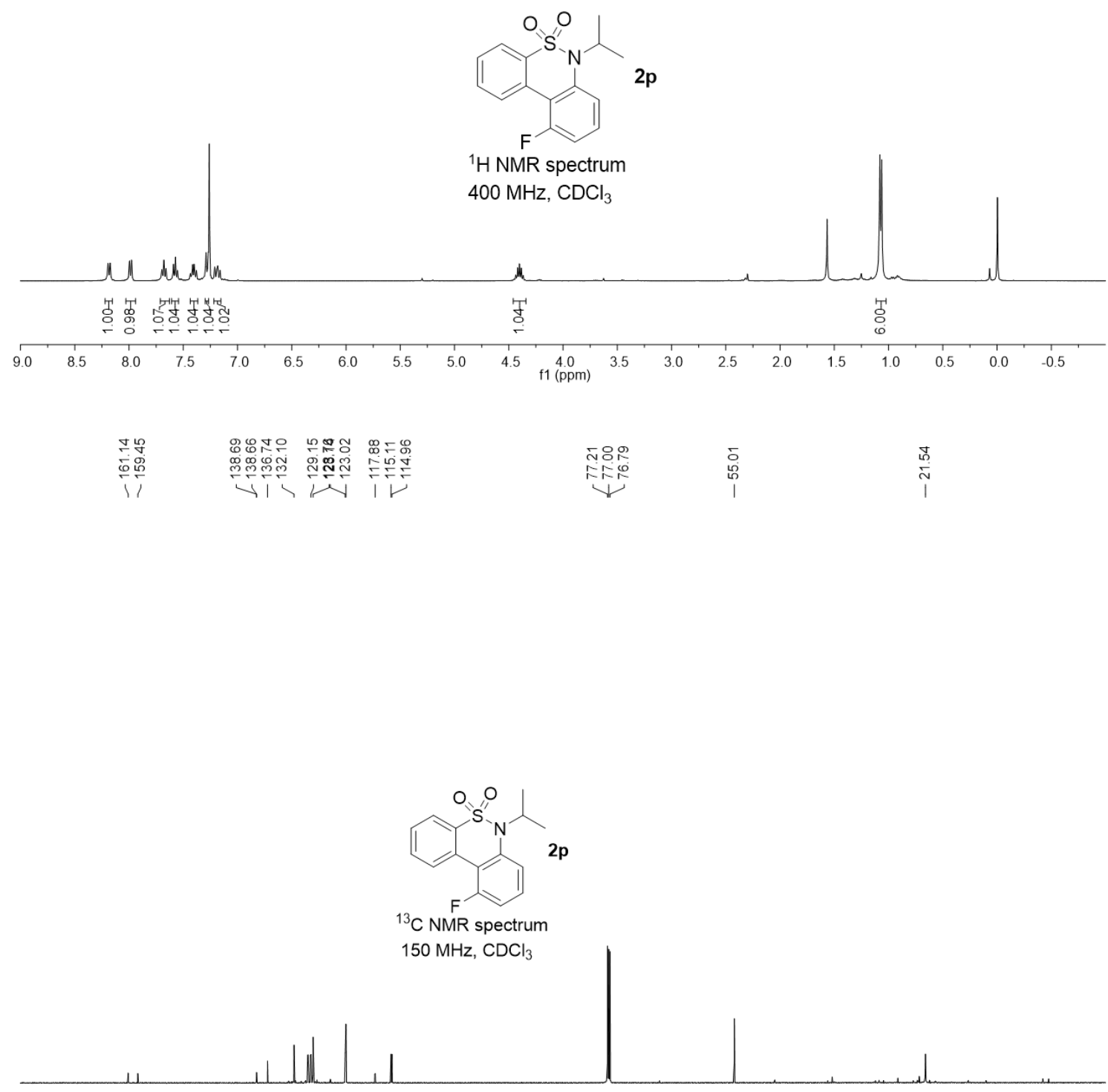

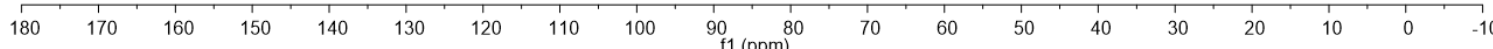




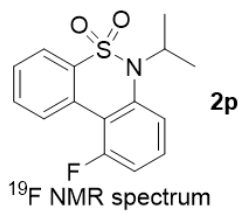

$375 \mathrm{MHz}, \mathrm{CDCl}_{3}$

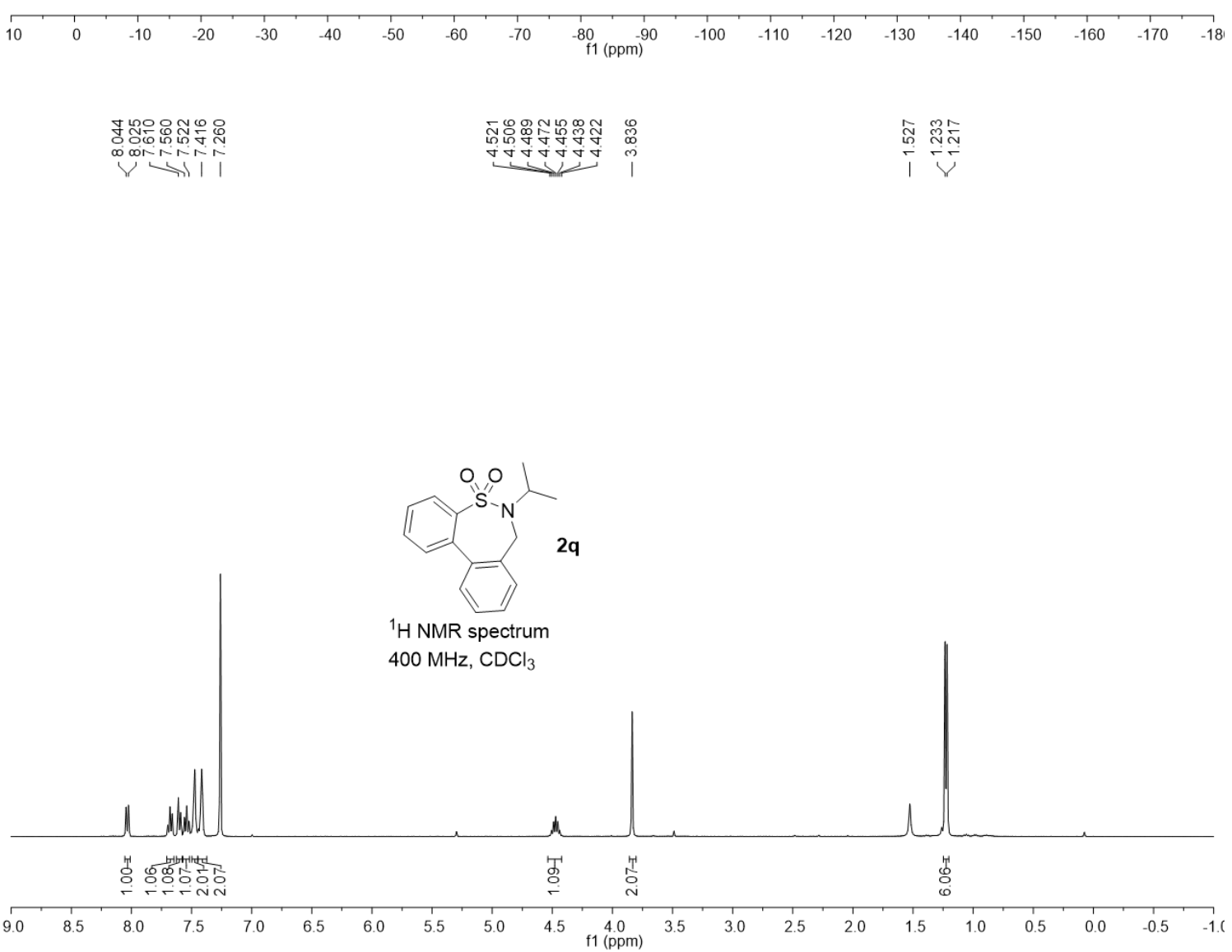



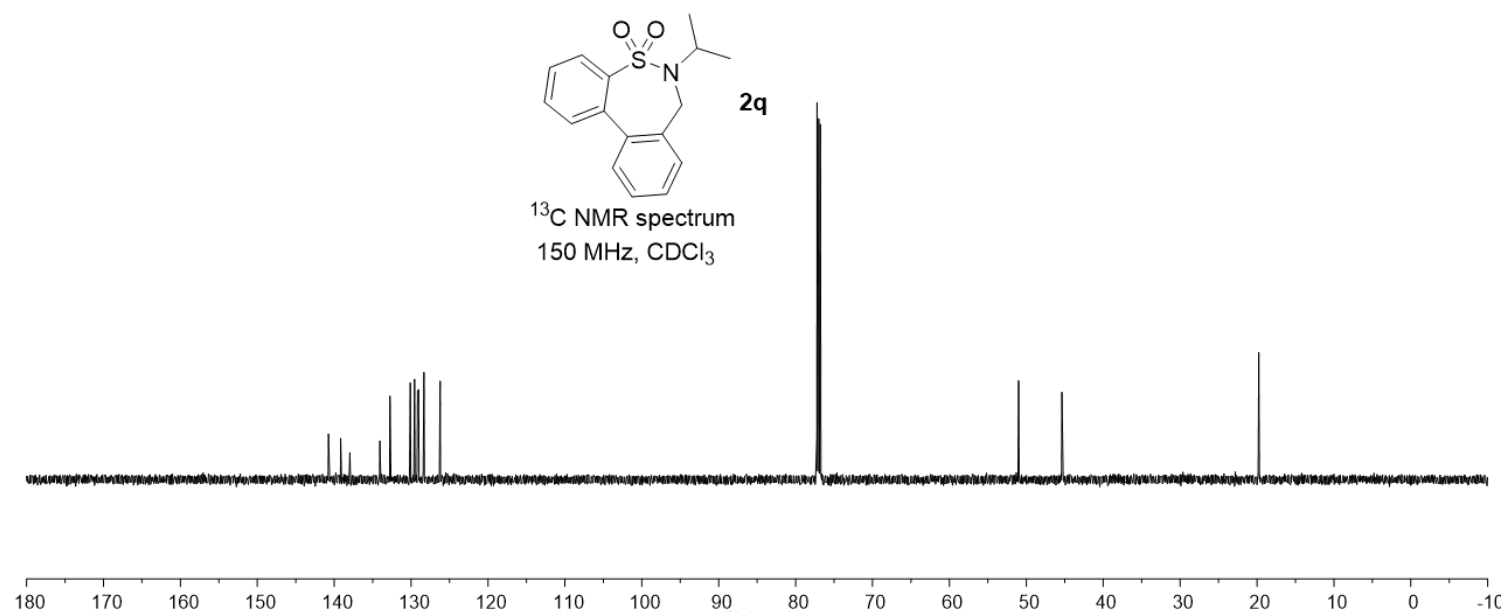

$90 \quad 80$

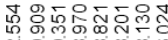

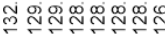

1 I

$\begin{array}{llll}8 & \infty & 0 & 0 \\ 0 & 0 & 0 & 5 \\ 1 & 00 & 0 & 0 \\ 1 & 1 & 1 & j\end{array}$

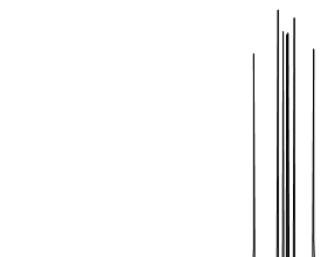

0,0

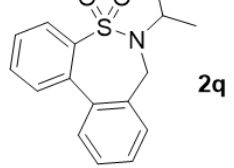

$2 q$

DEPT spectrum

$150 \mathrm{MHz}, \mathrm{CDCl}_{3}$

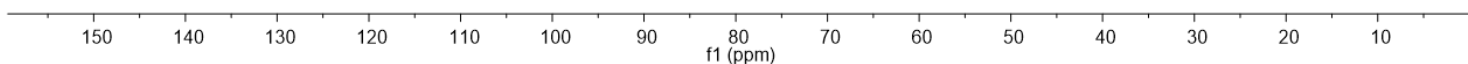



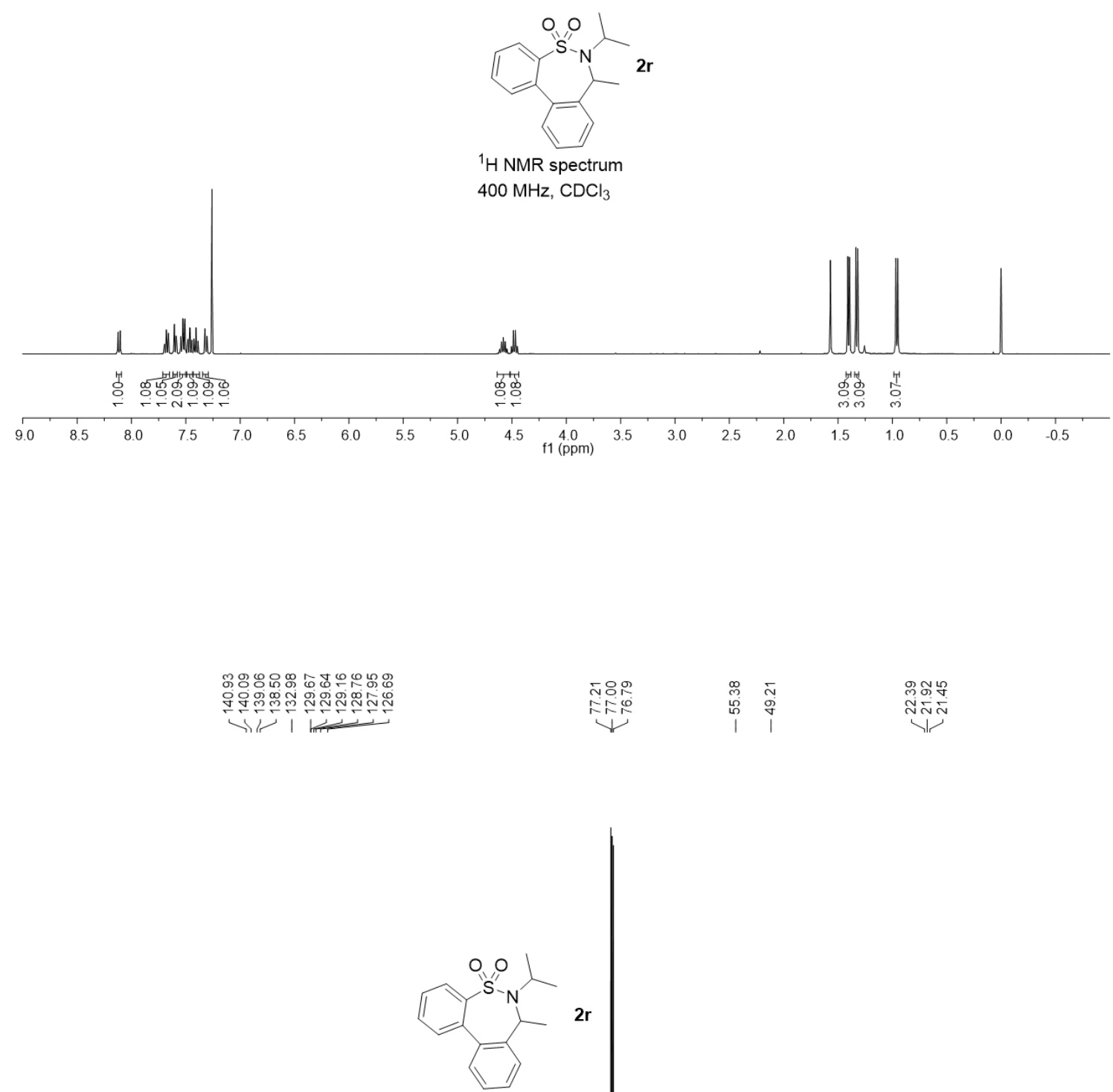

${ }^{13} \mathrm{C}$ NMR spectrum $150 \mathrm{MHz}, \mathrm{CDCl}_{3}$

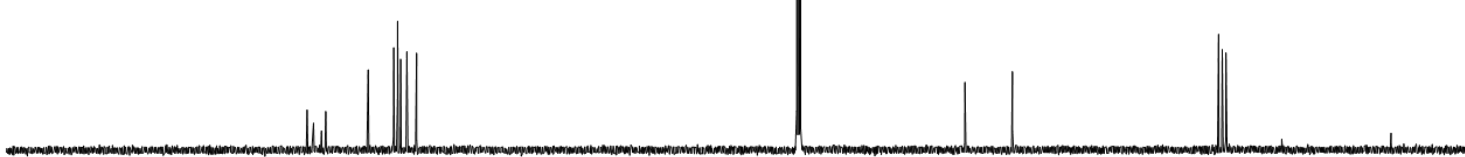




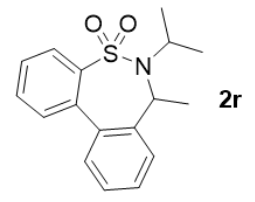

DEPT spectrum

$100 \mathrm{MHz}, \mathrm{CDCl} 3$

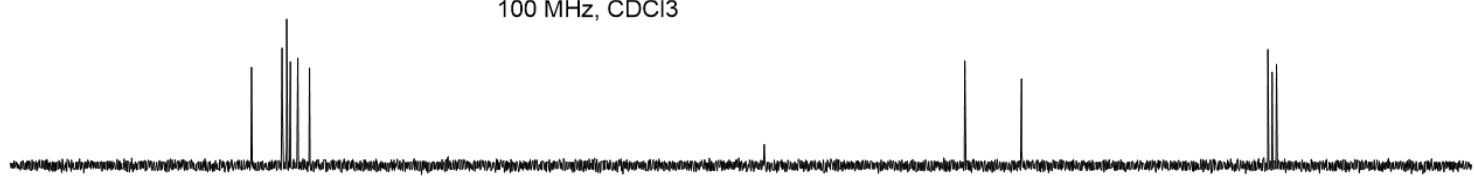

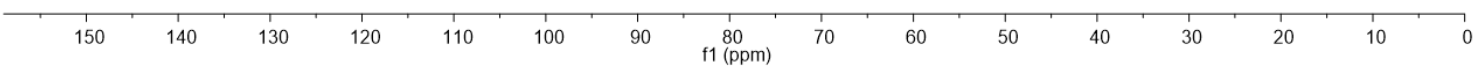

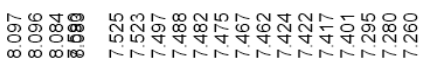

票

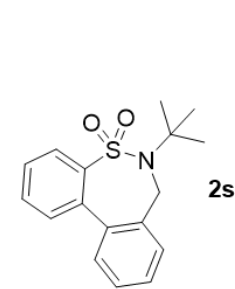

${ }^{1} \mathrm{H}$ NMR spectrum

$600 \mathrm{MHz}, \mathrm{CDCl}_{3}$
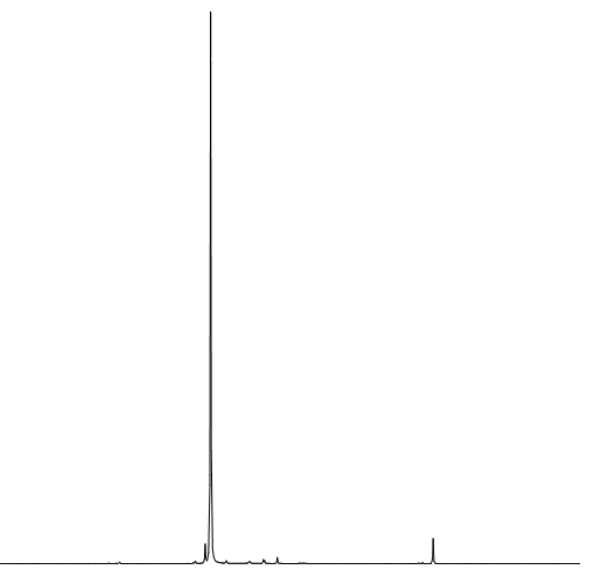

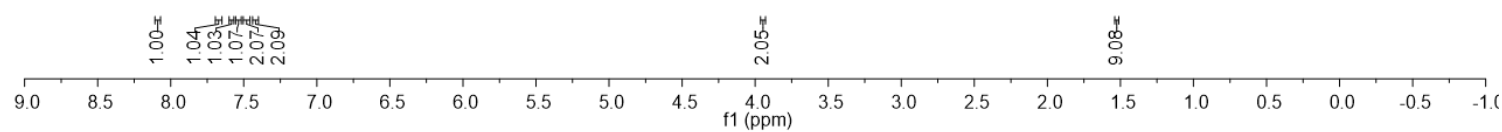



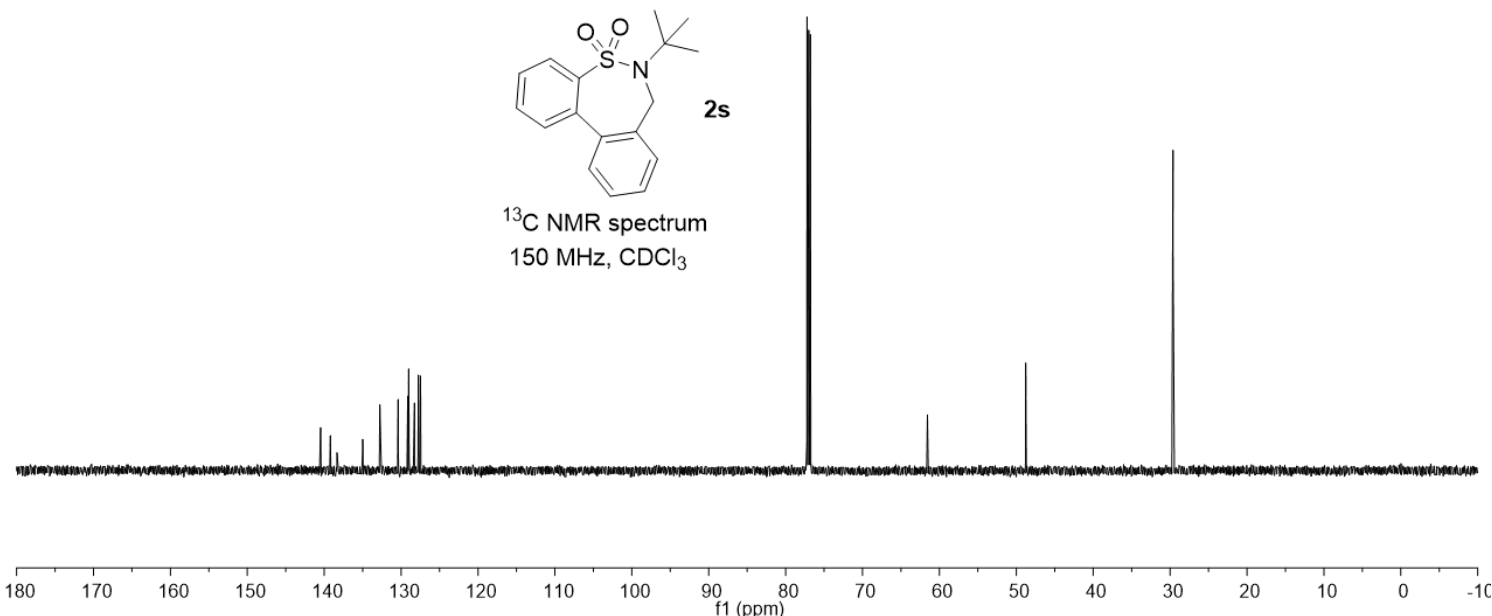

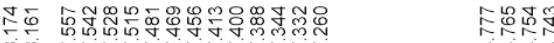

$\sqrt{\infty}$

㹂

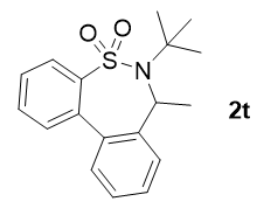

${ }^{1} \mathrm{H}$ NMR spectrum

$600 \mathrm{MHz} \mathrm{CDCl}_{3}$

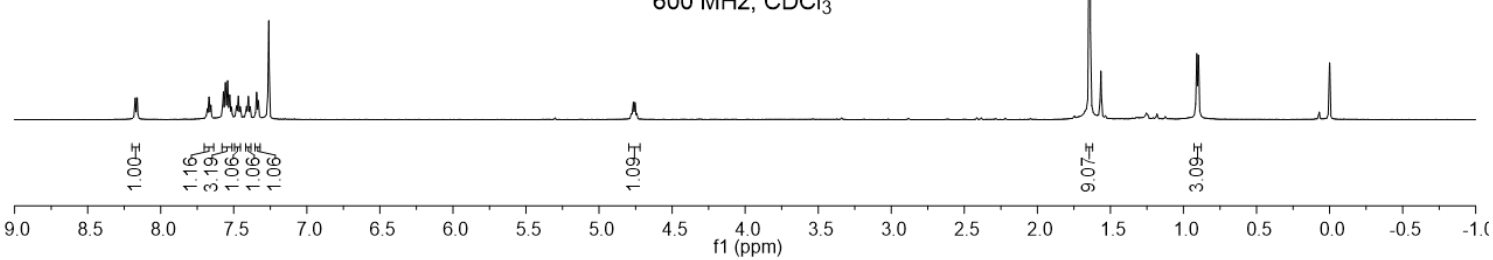



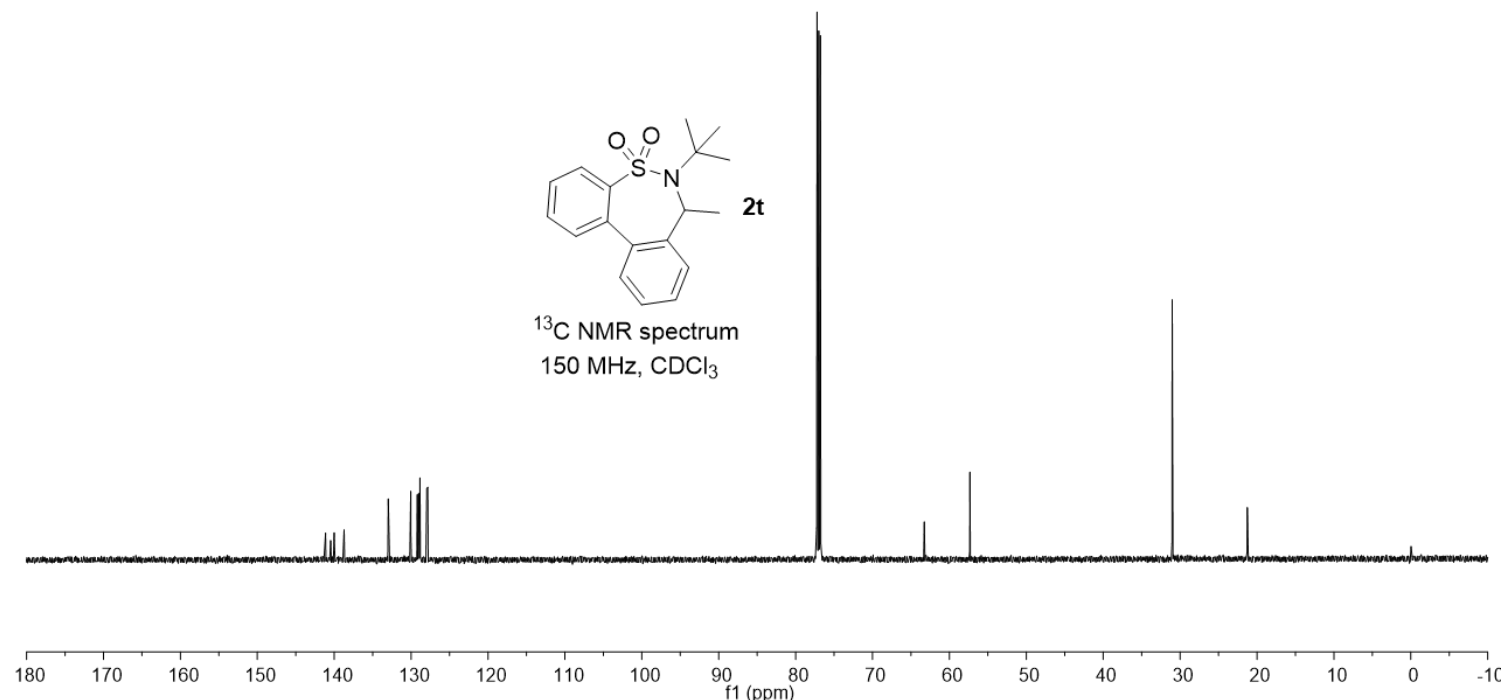

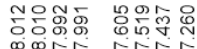
皮每

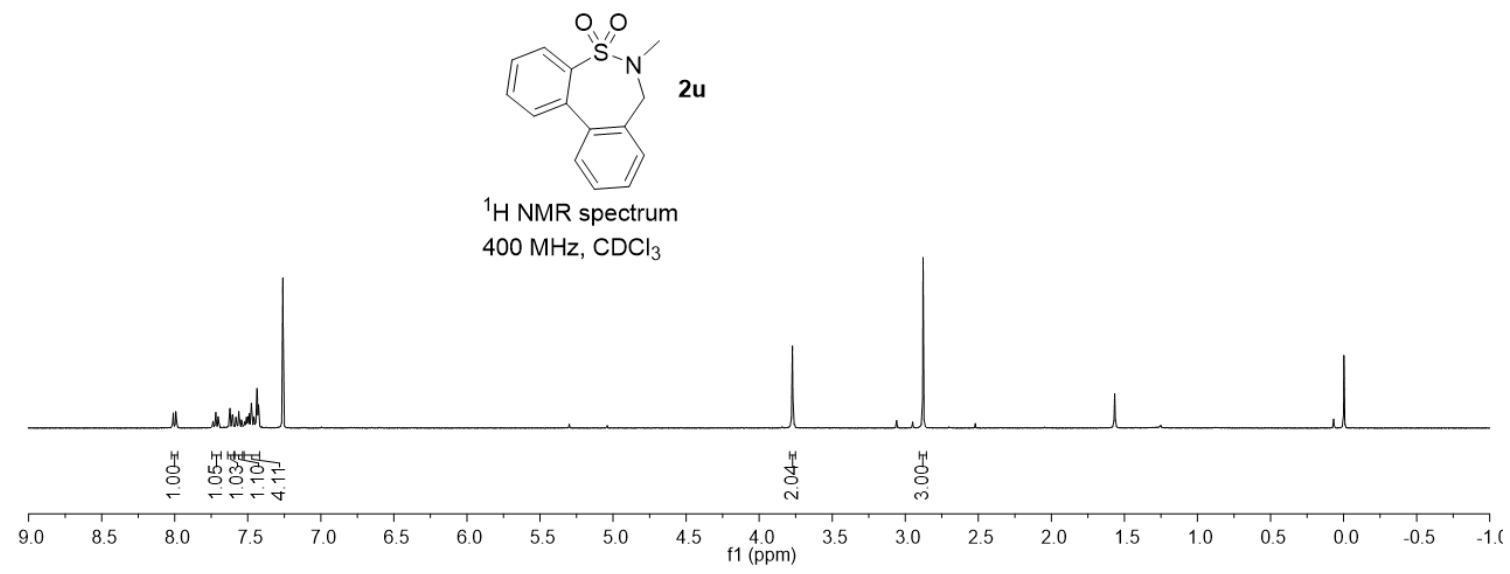



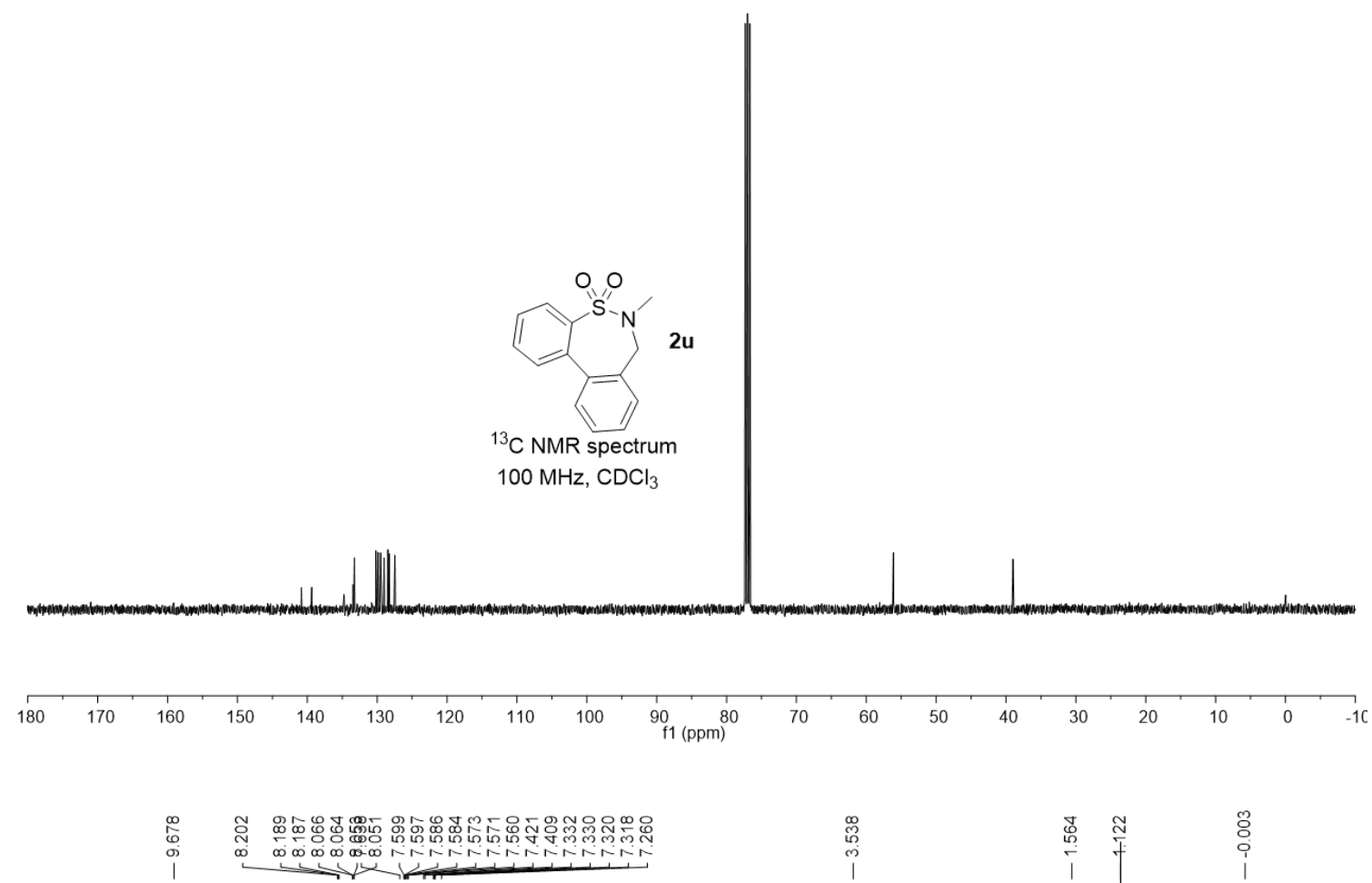



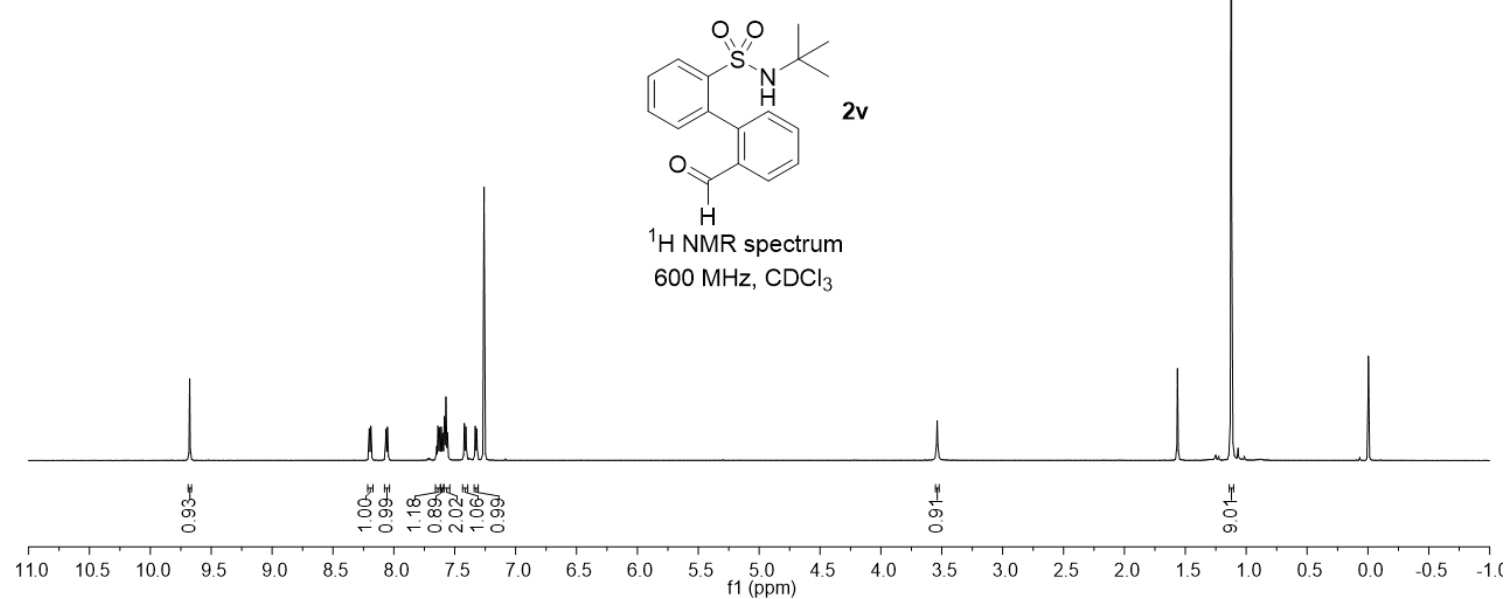




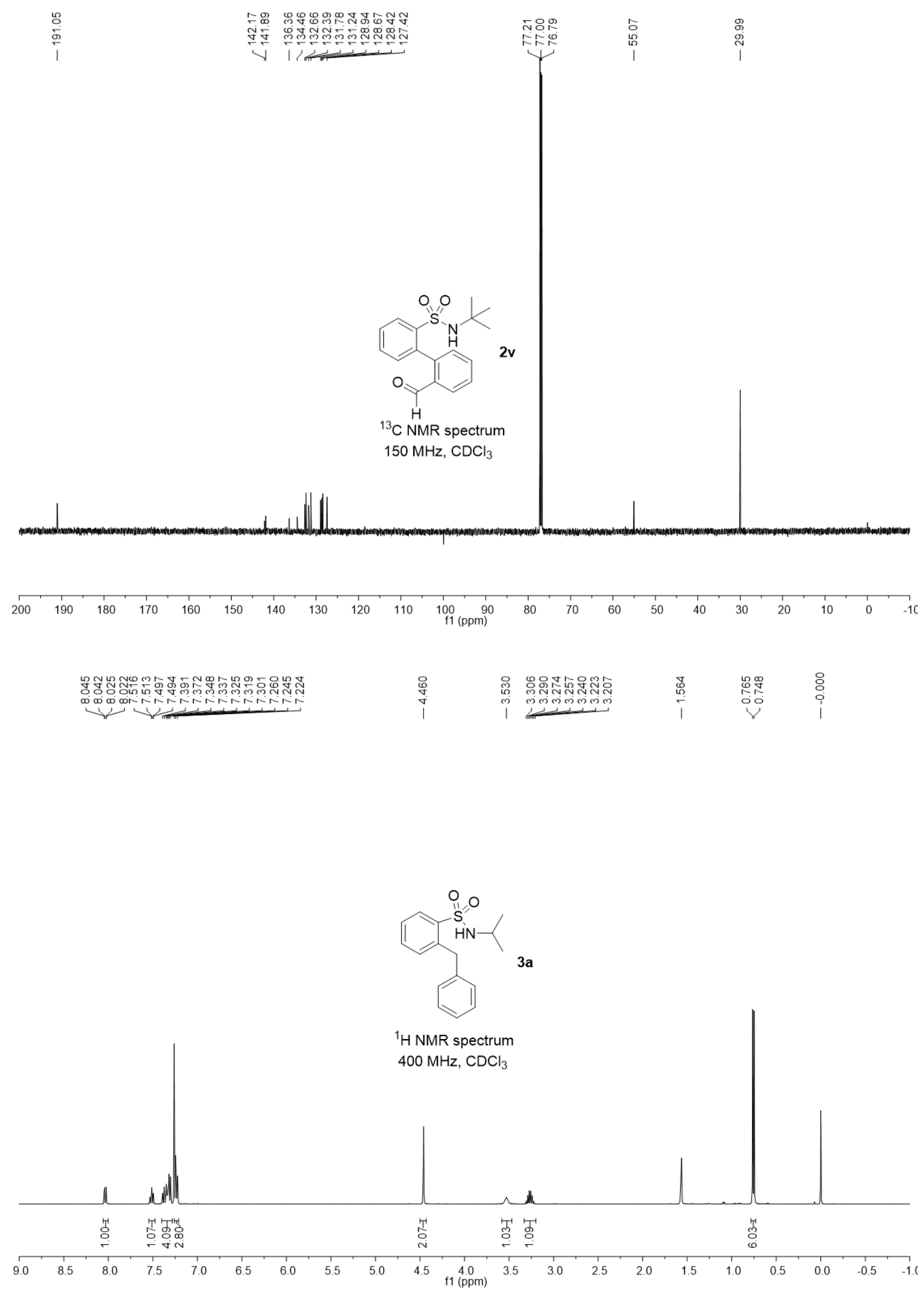



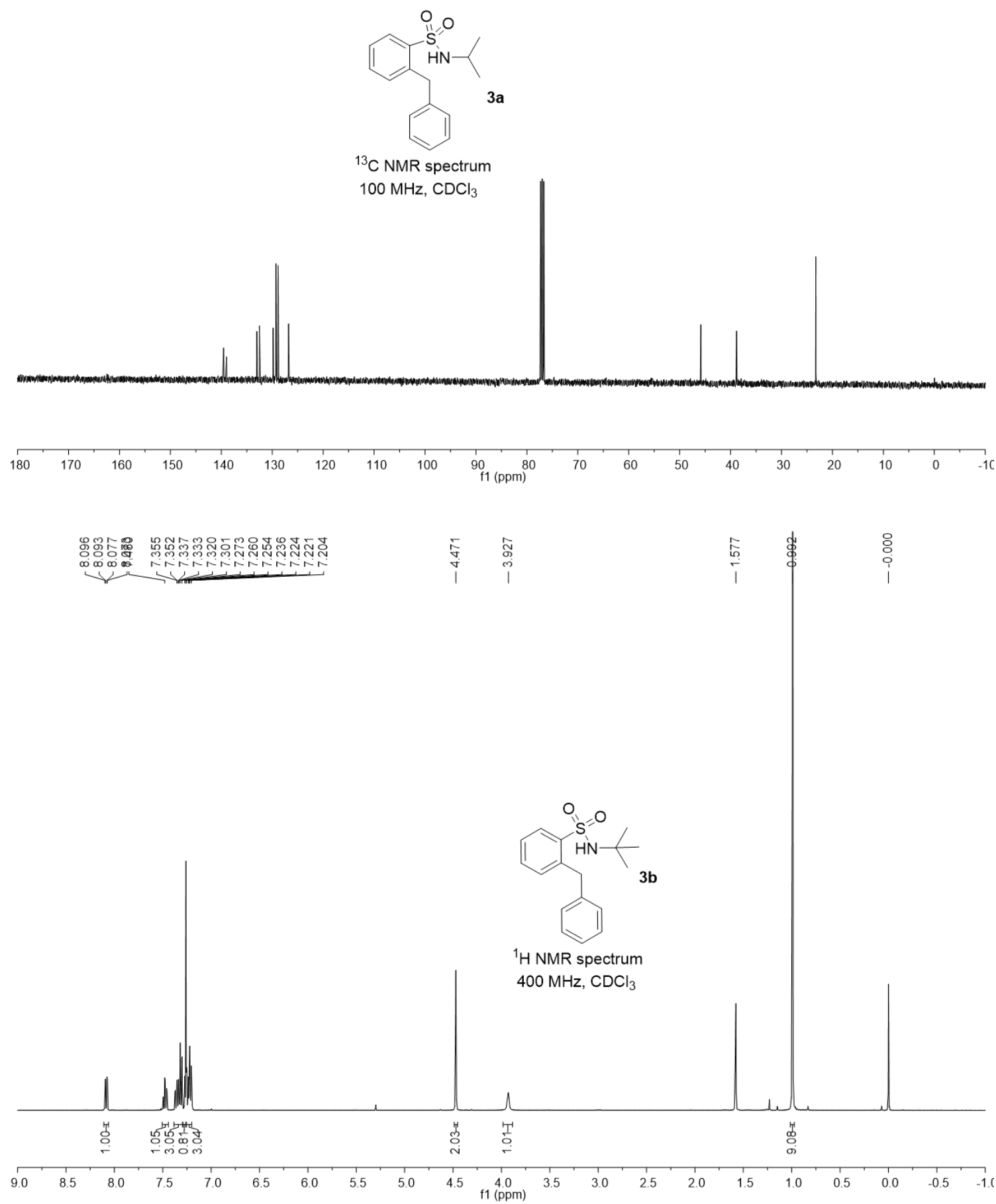


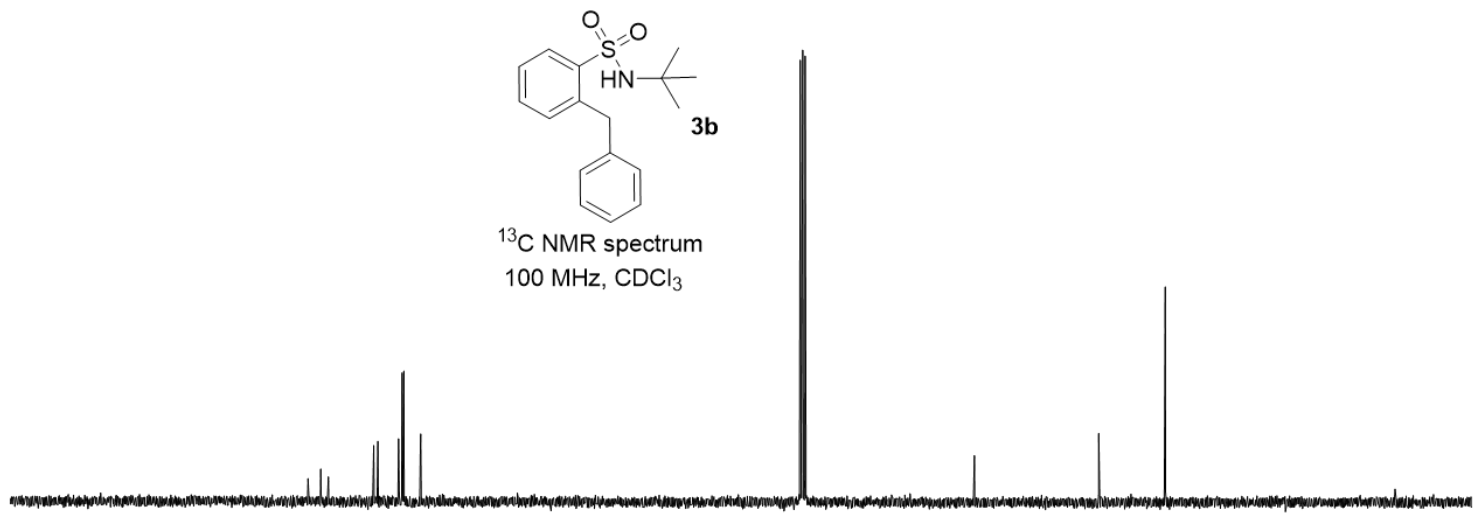

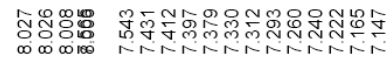

ป

o̊

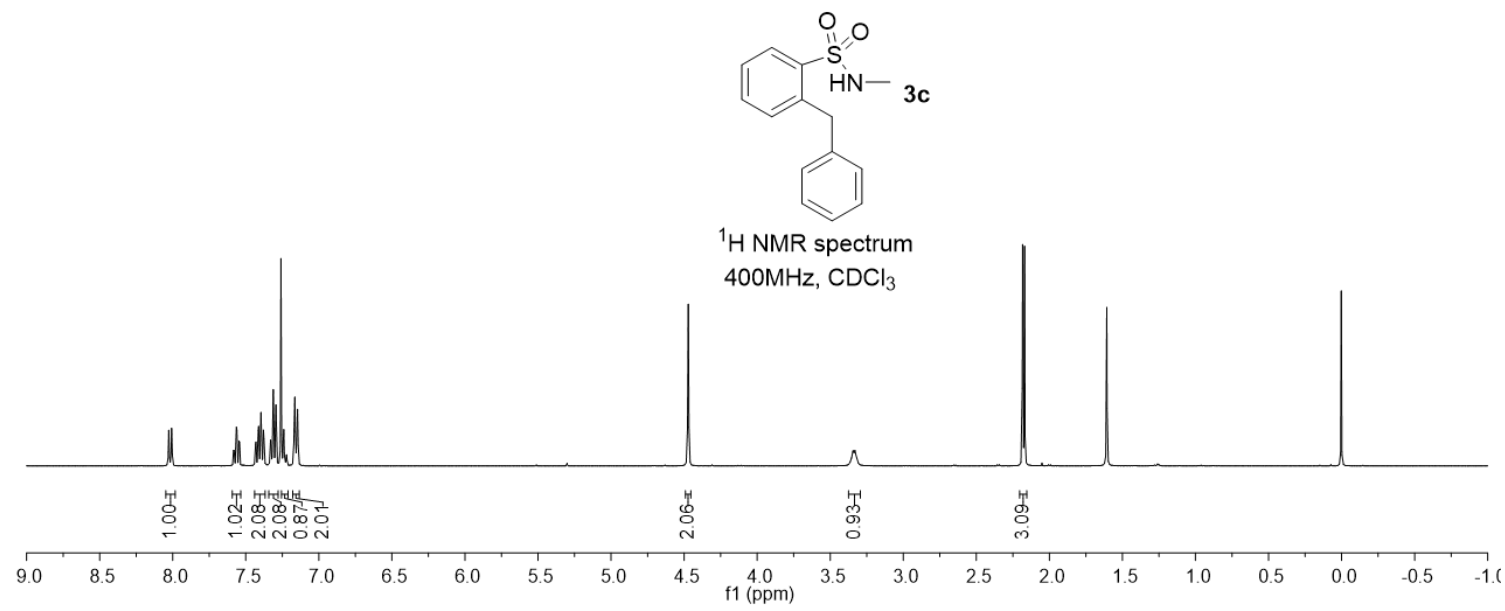



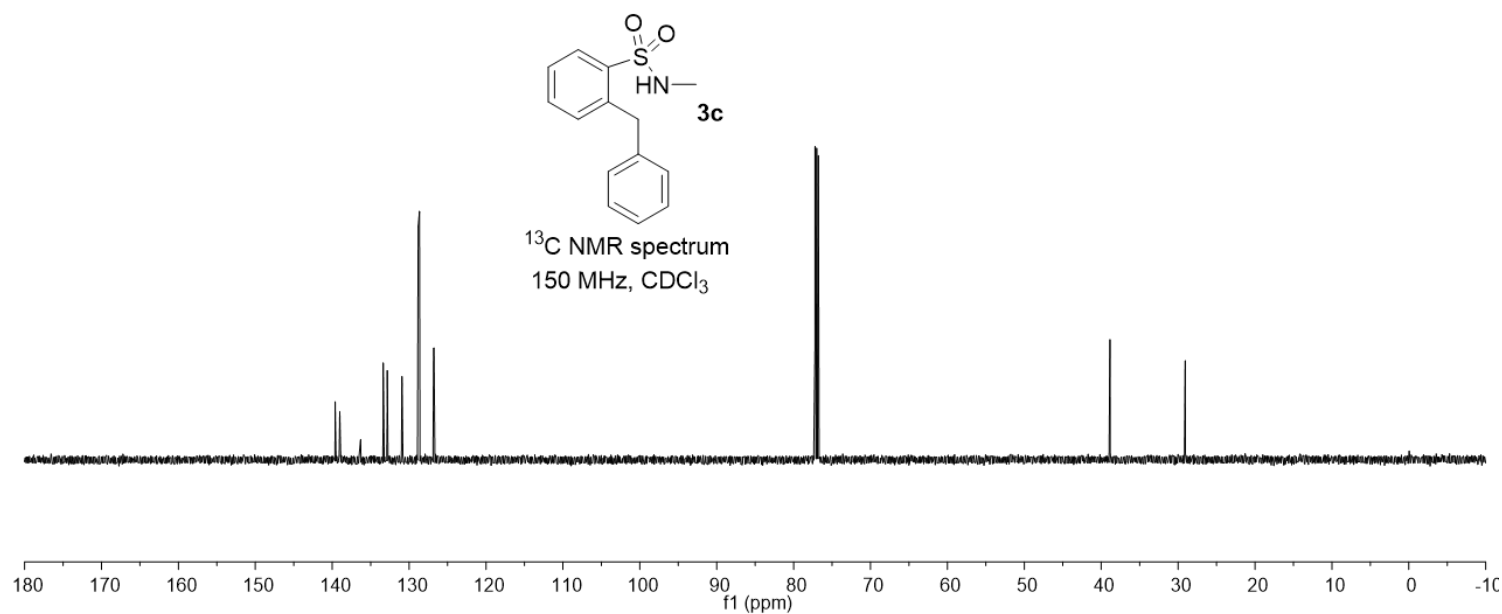

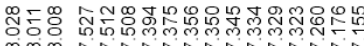

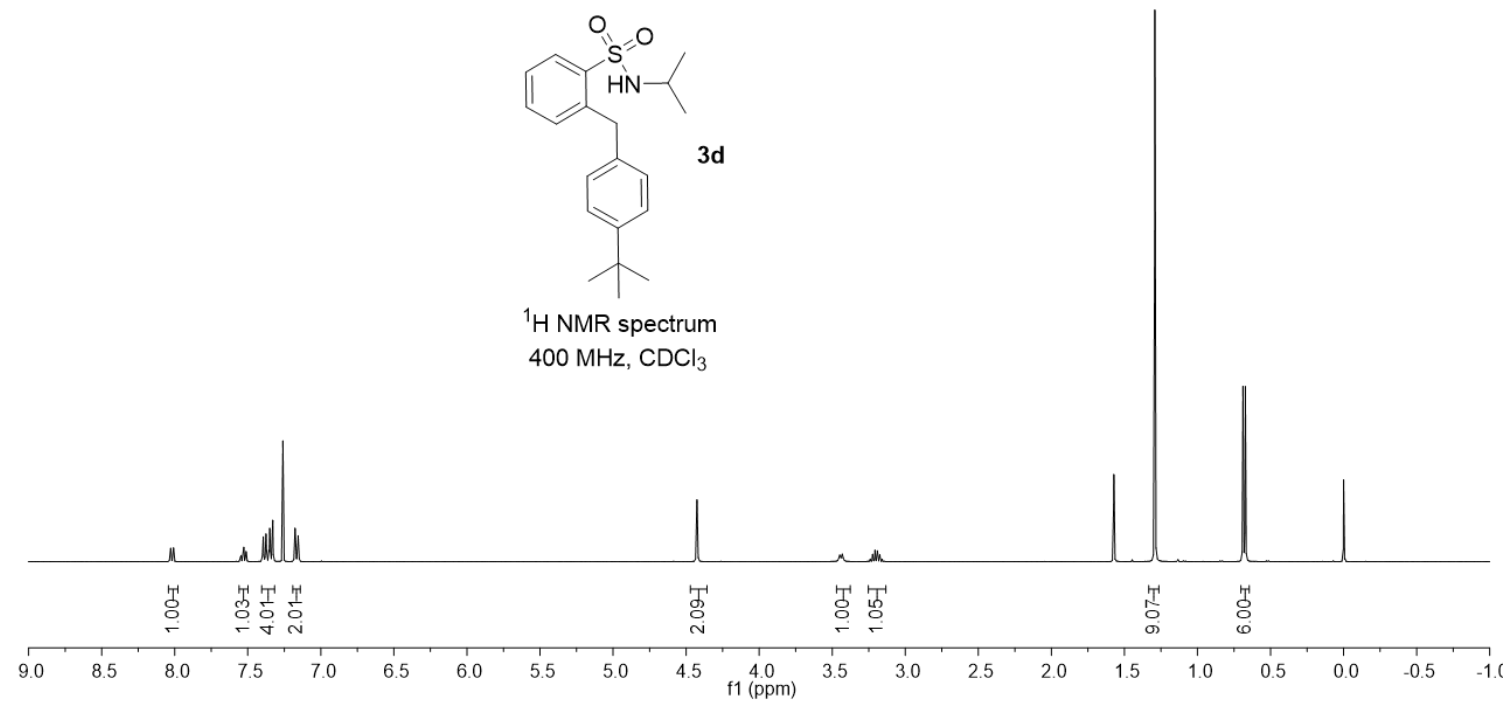



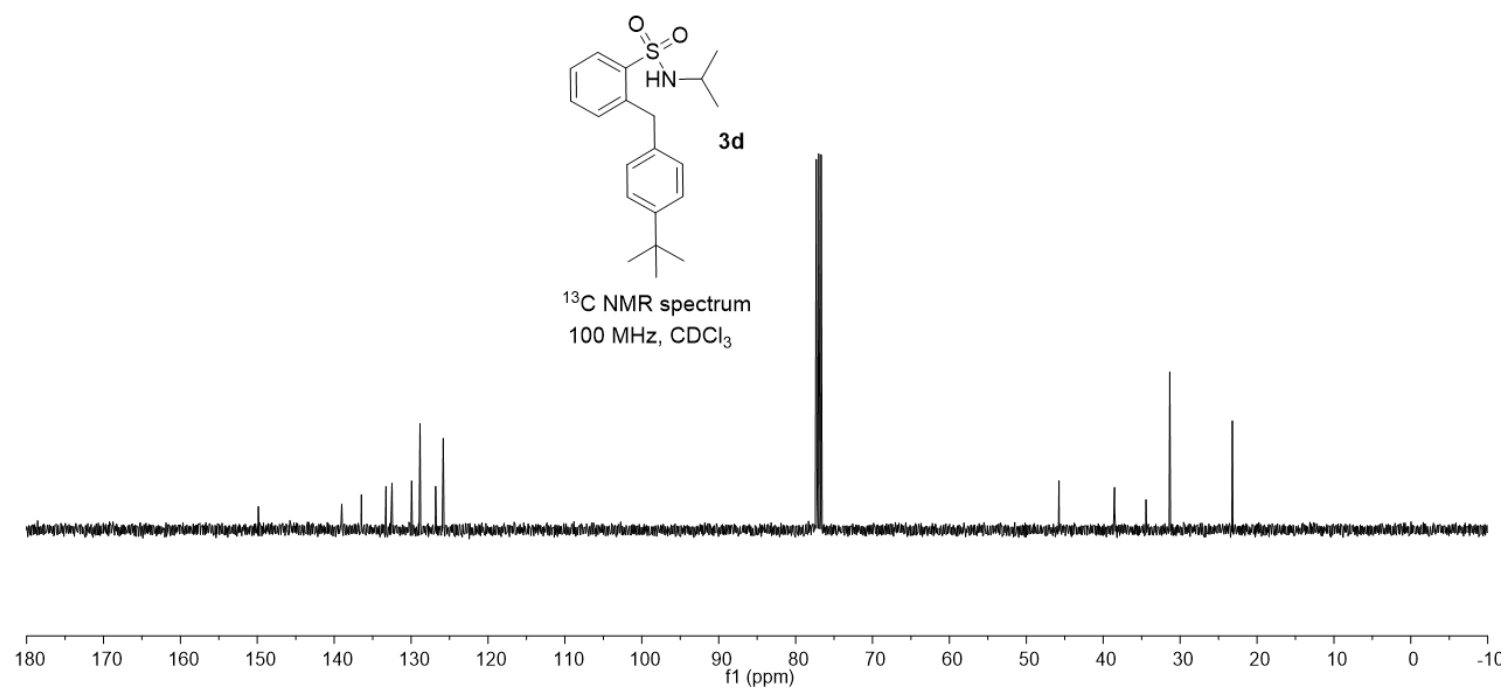

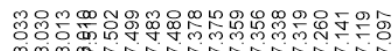

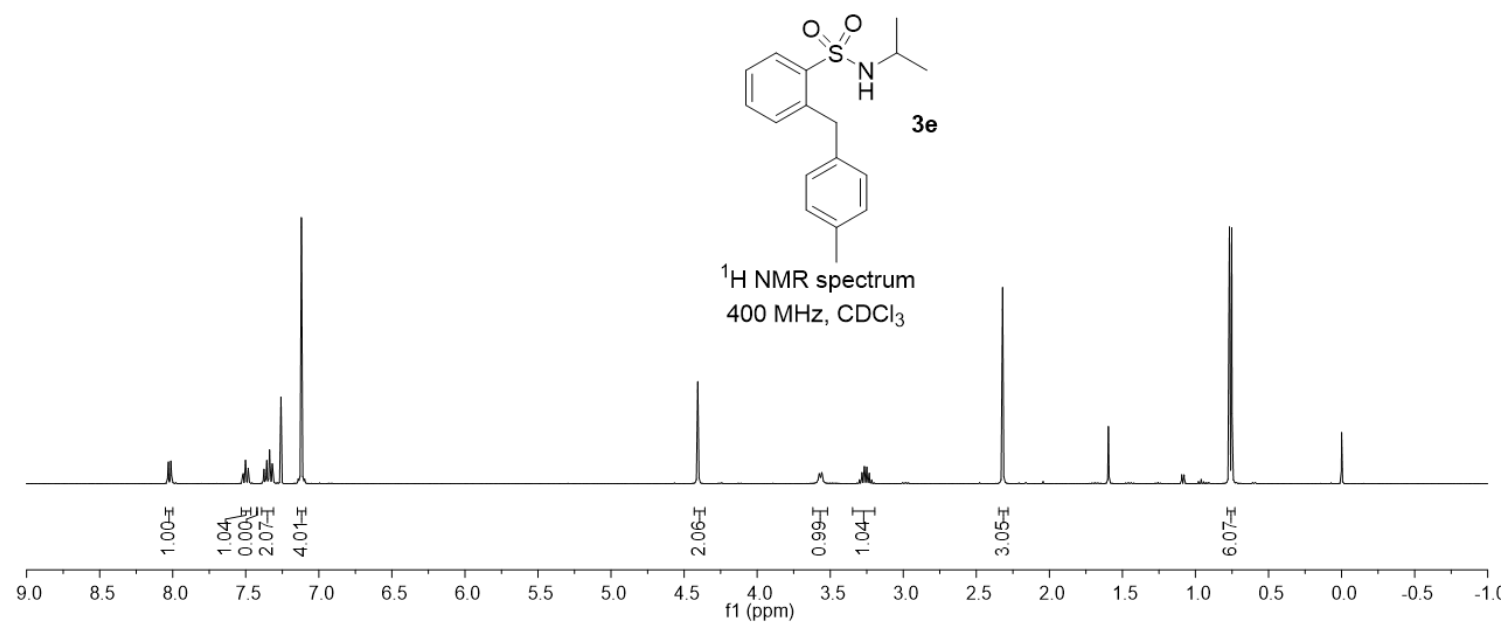




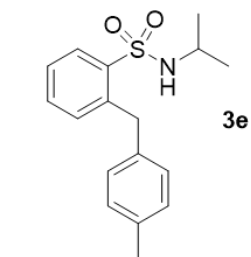

$3 e$ $100 \mathrm{MHz} \mathrm{CDCl}_{3}$
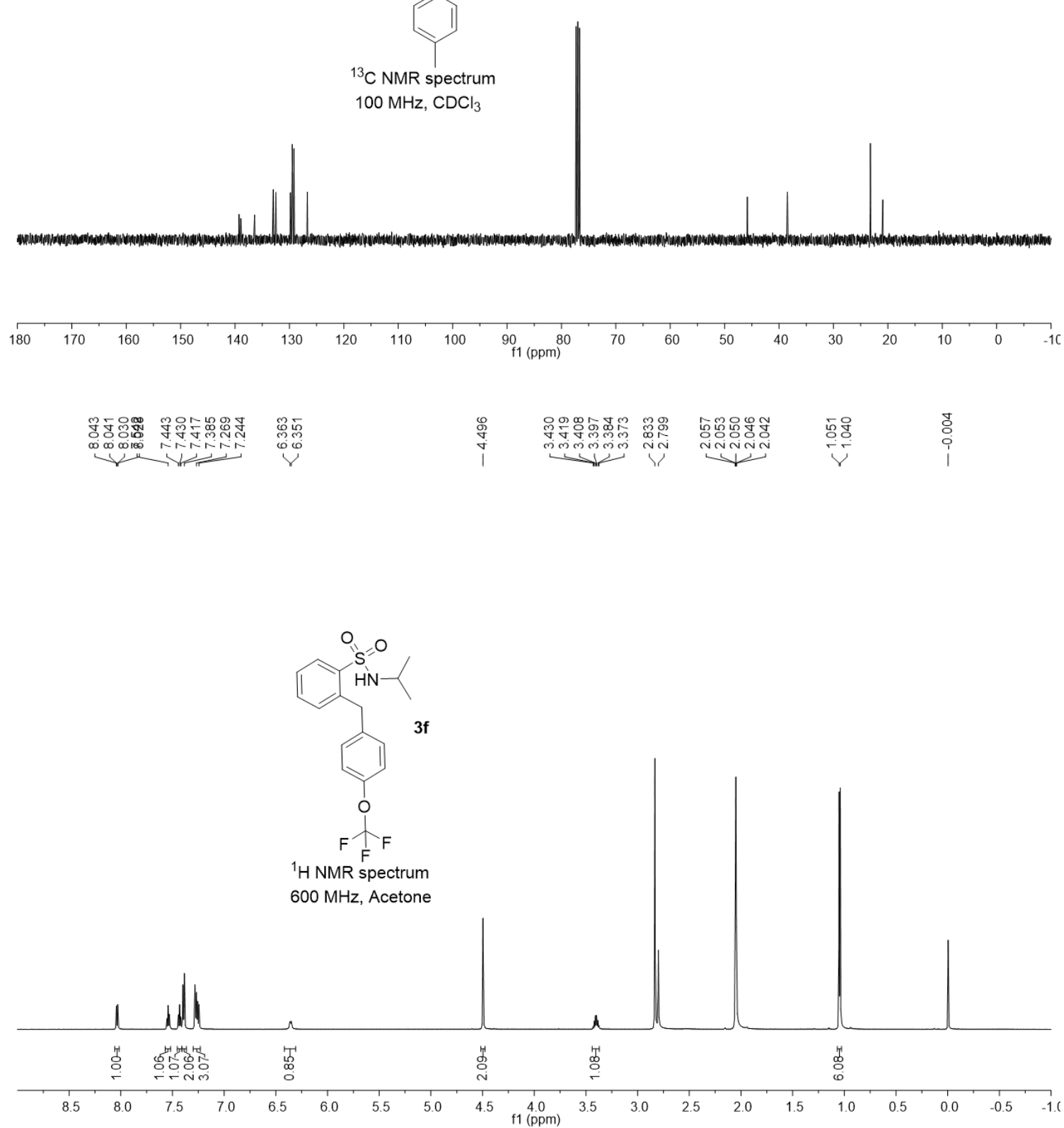

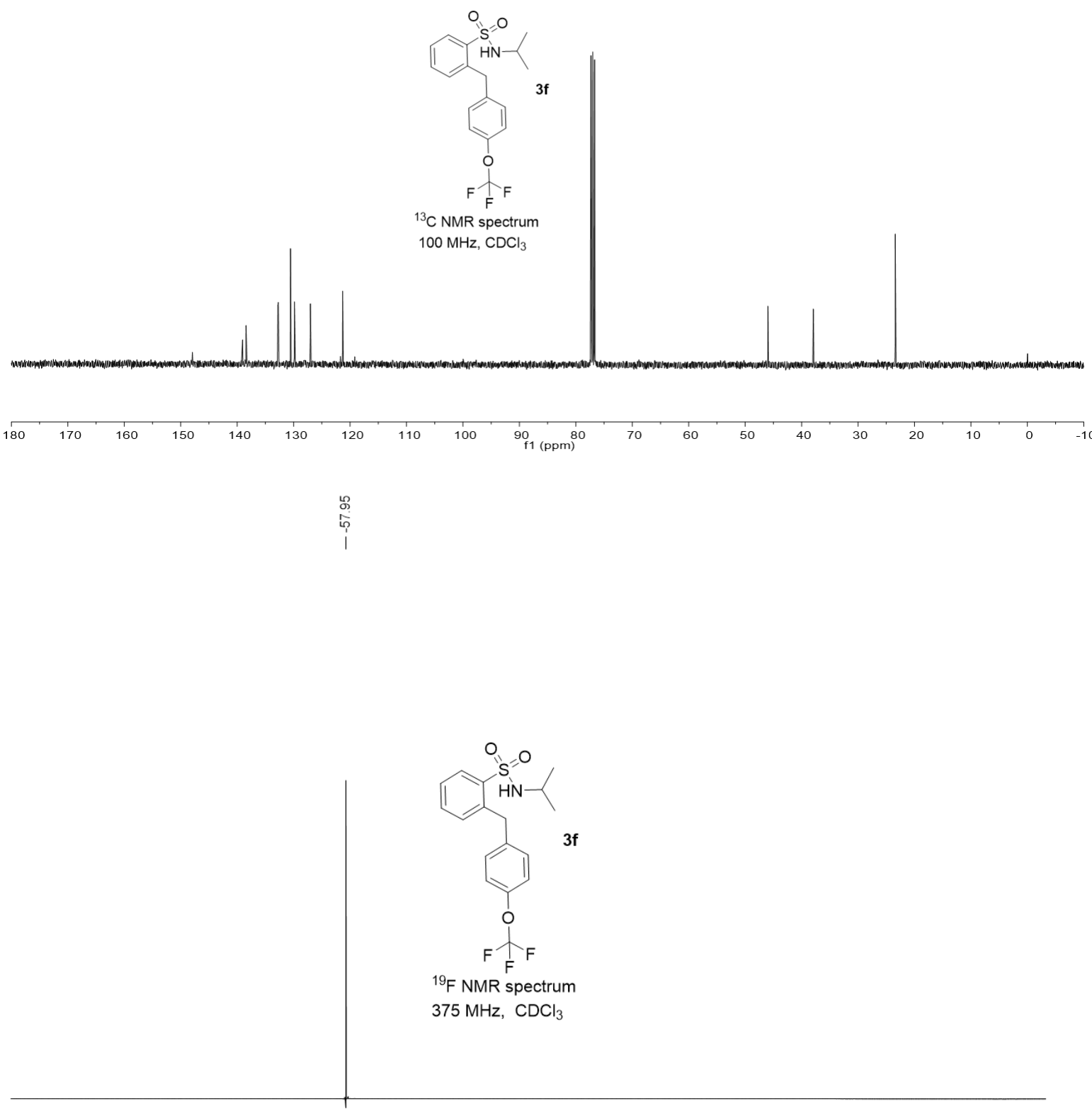

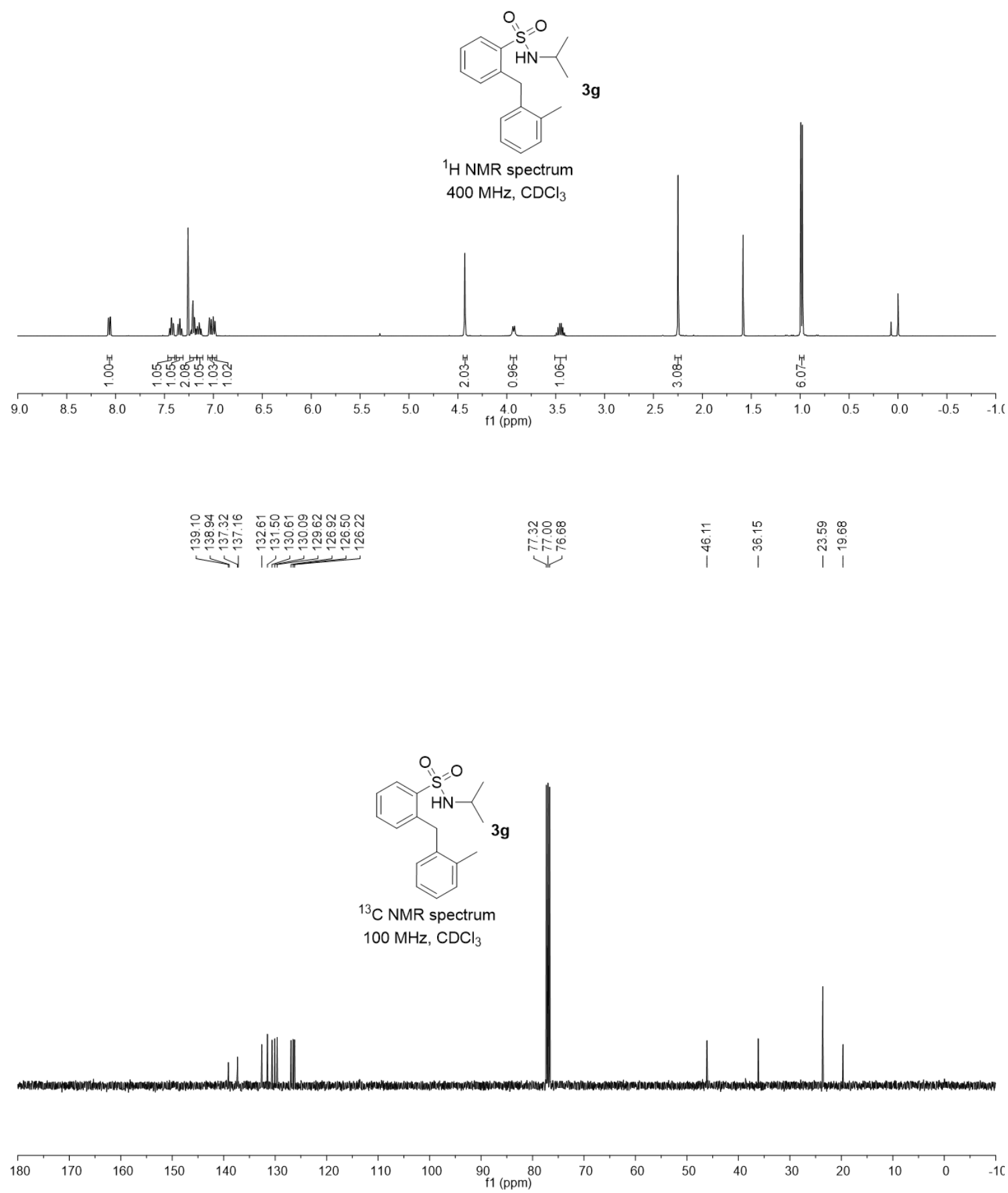

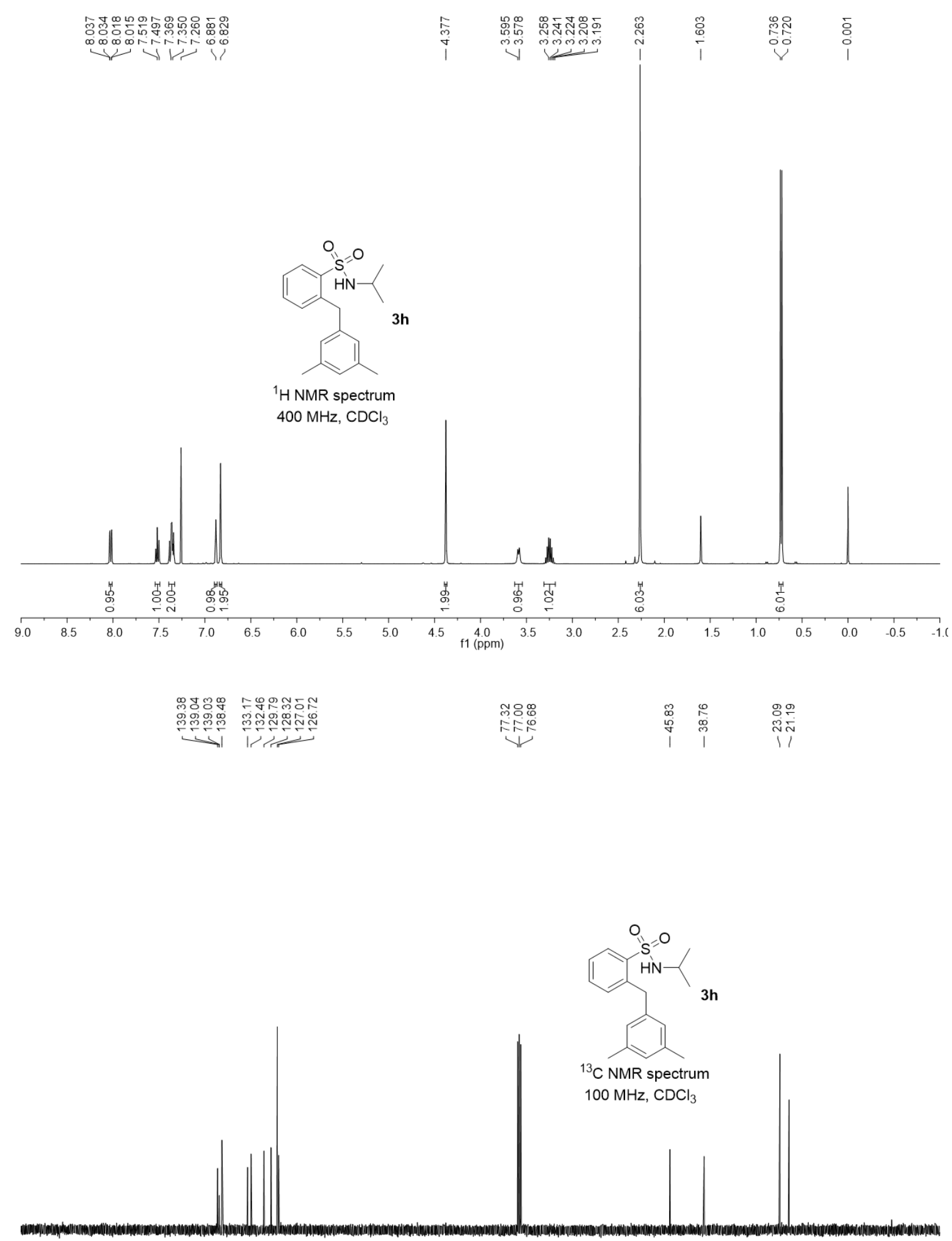

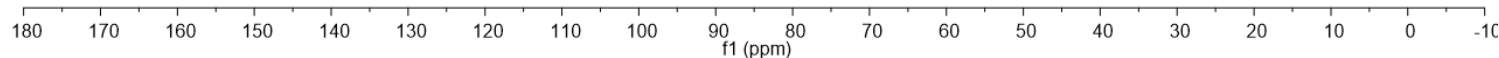



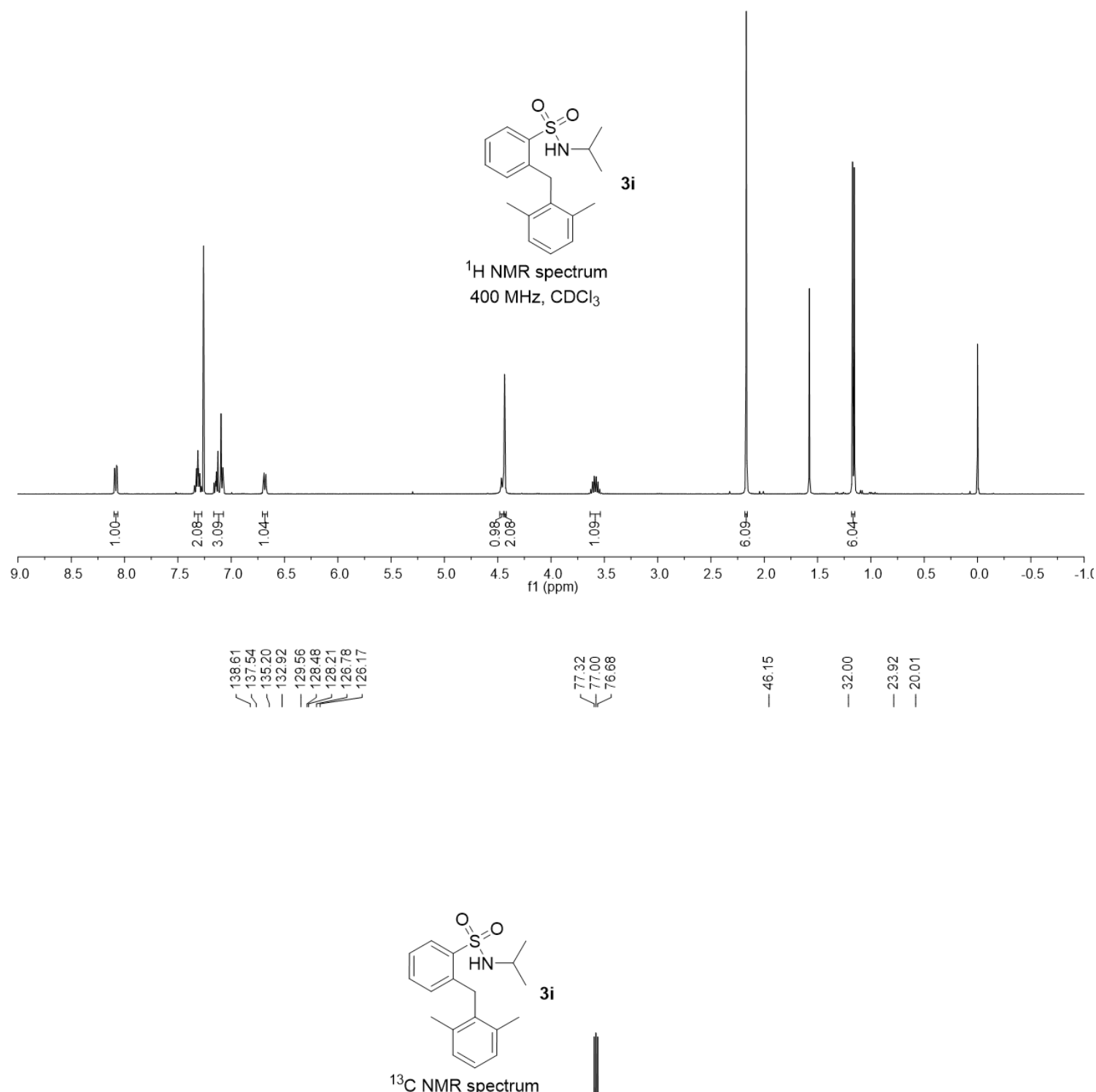

$100 \mathrm{MHz} \mathrm{CDCl}_{3}$ 


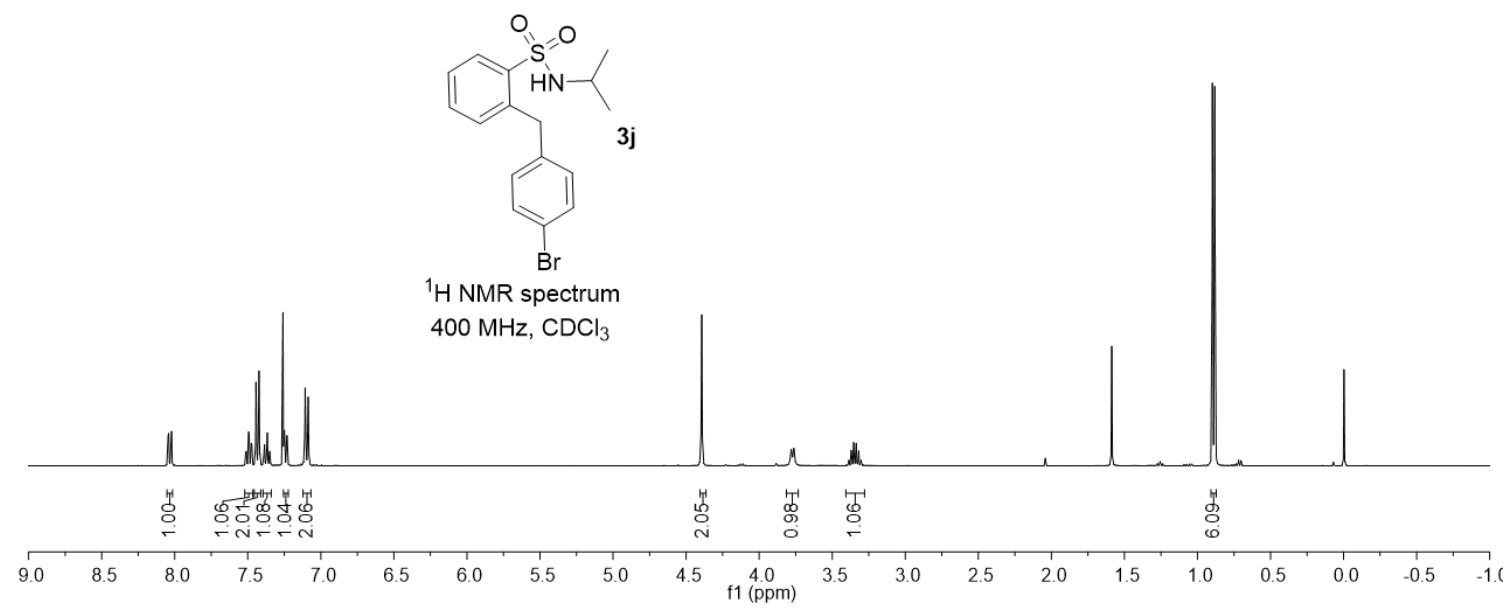

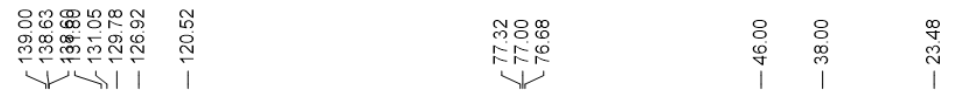

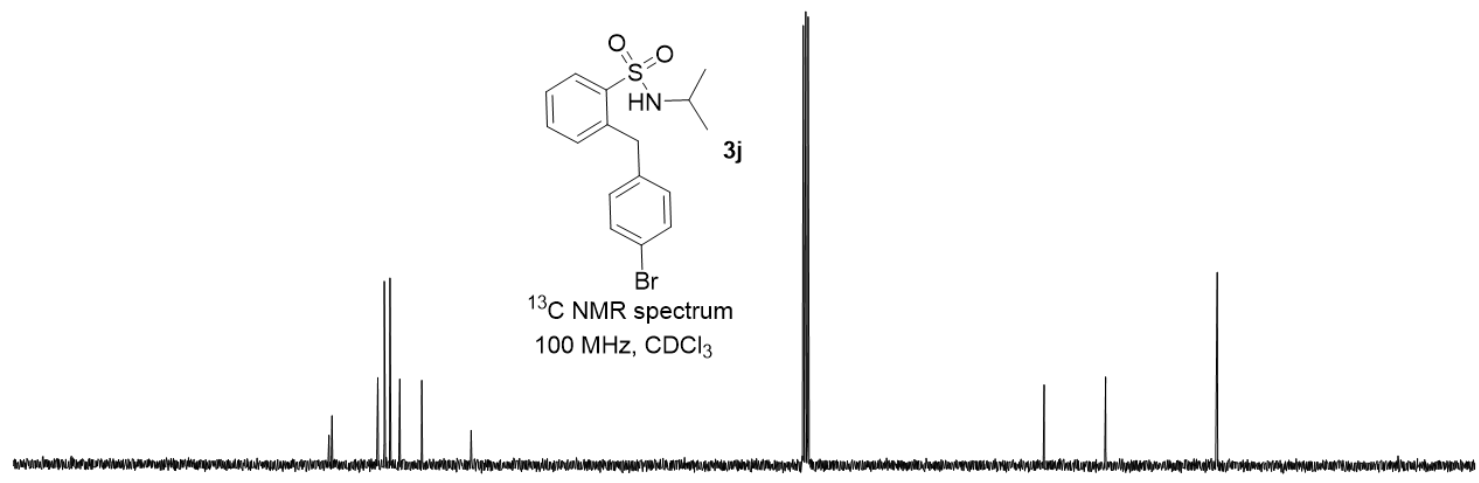

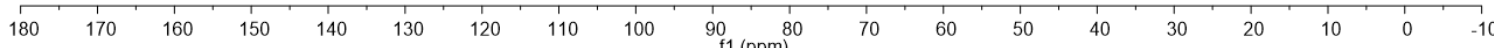




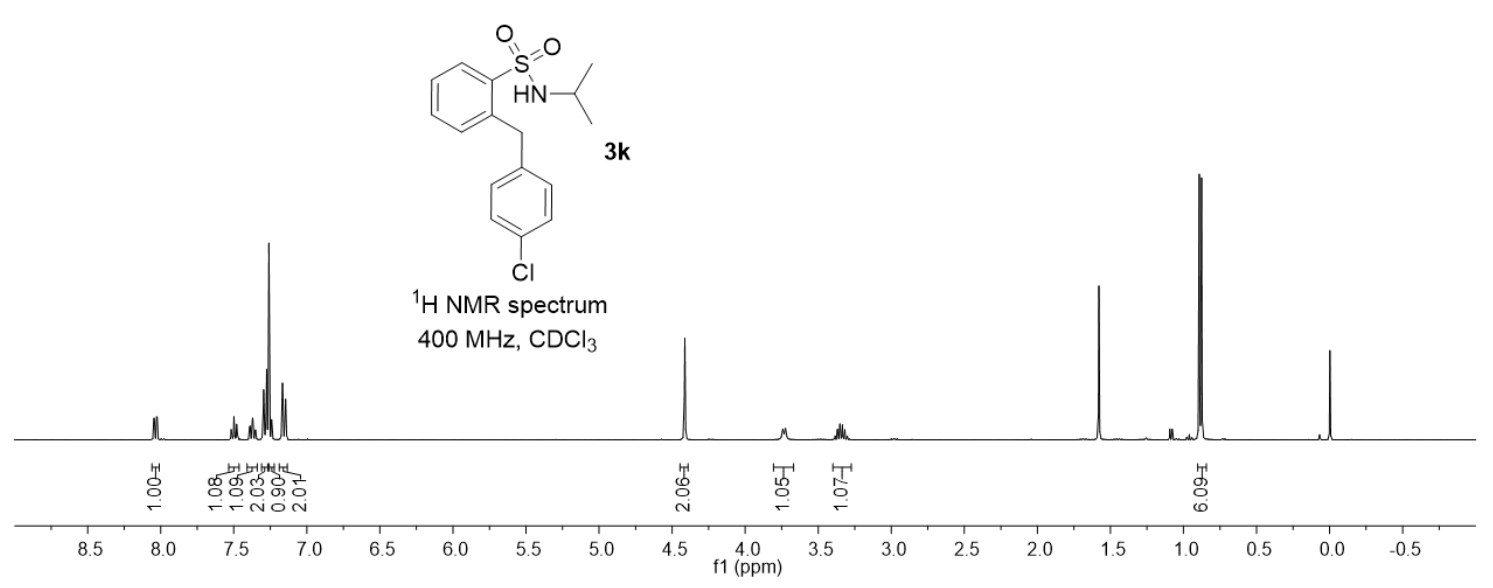

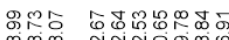

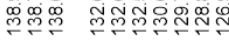

인

$\sqrt{1+1}$

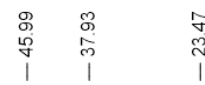

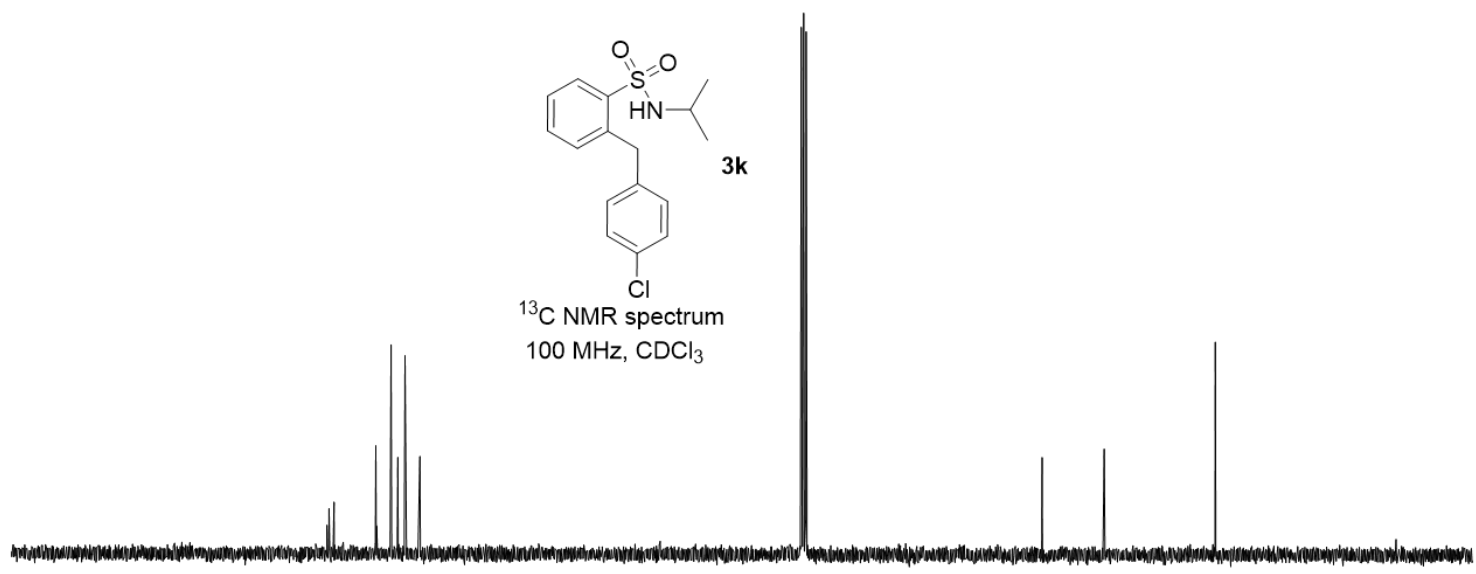

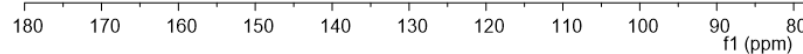




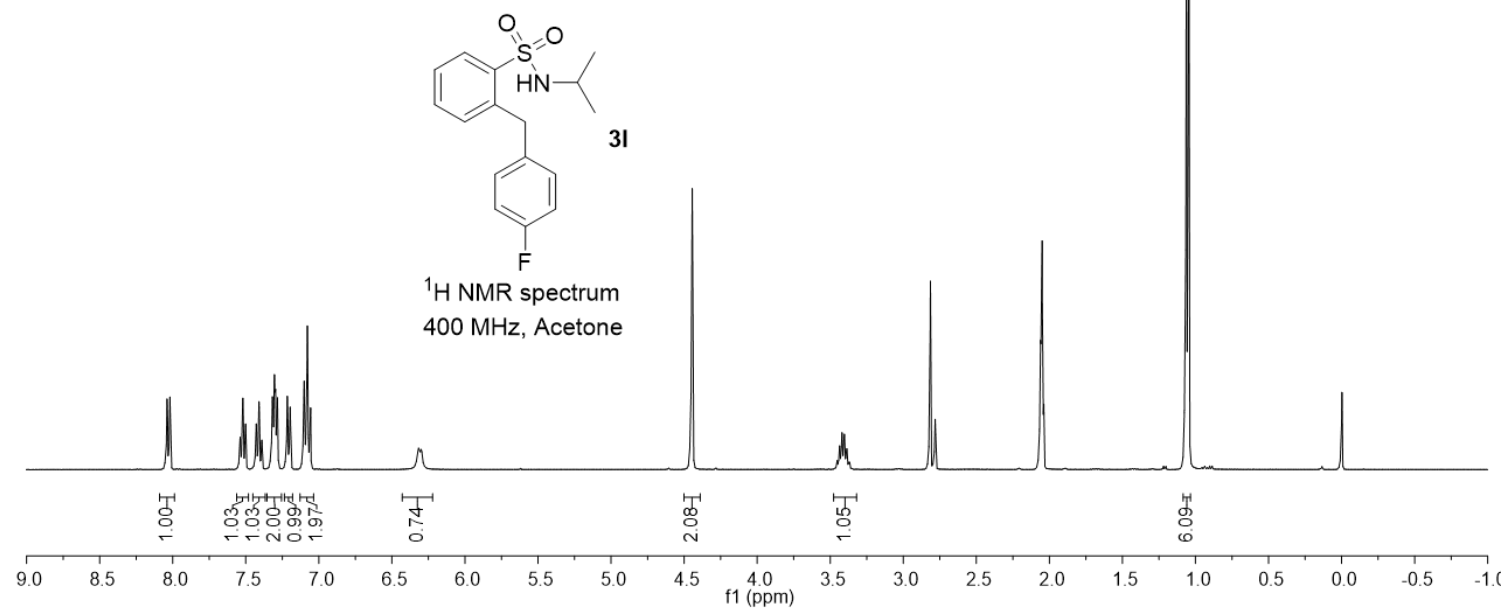

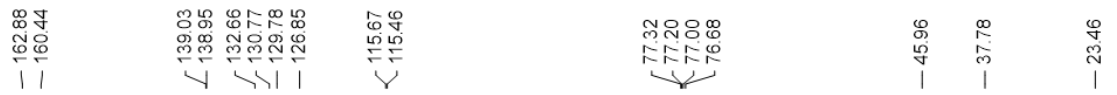

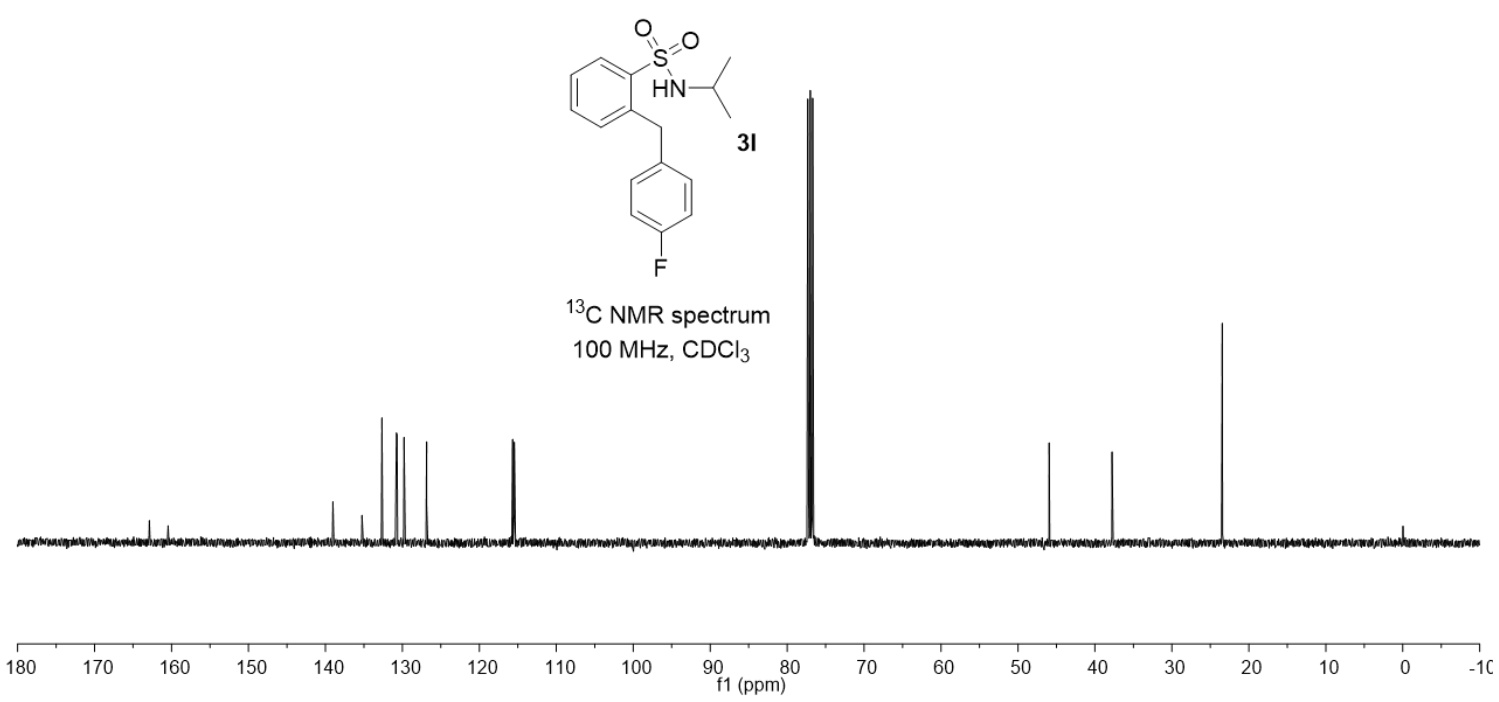




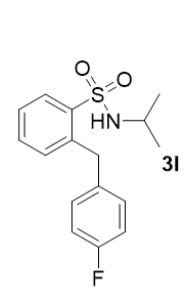

${ }^{19} \mathrm{~F}$ NMR spectrum $375 \mathrm{MHz}$, Acetone

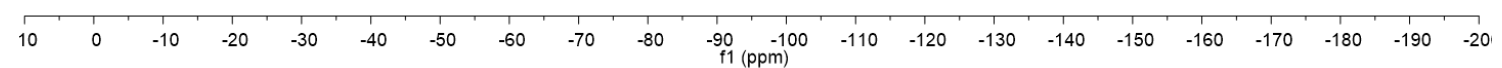

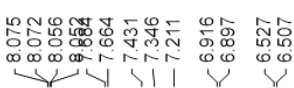

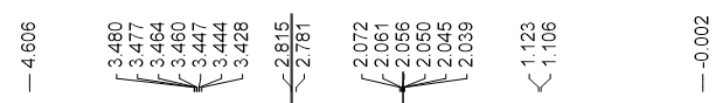

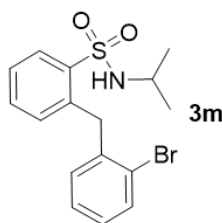

${ }^{1} \mathrm{H}$ NMR spectrum $400 \mathrm{MHz}$, Acetone
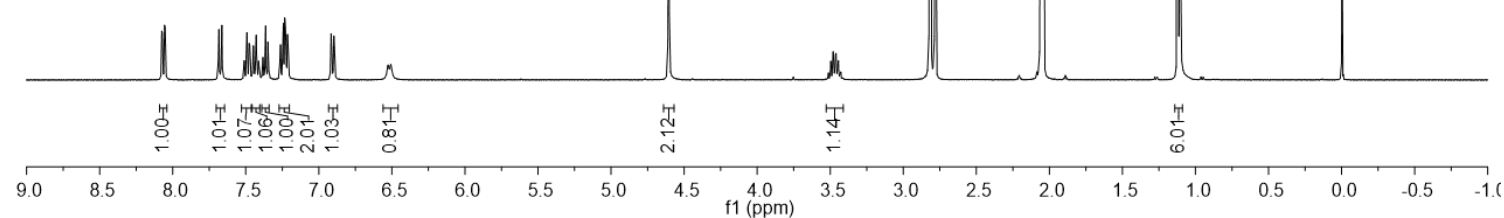

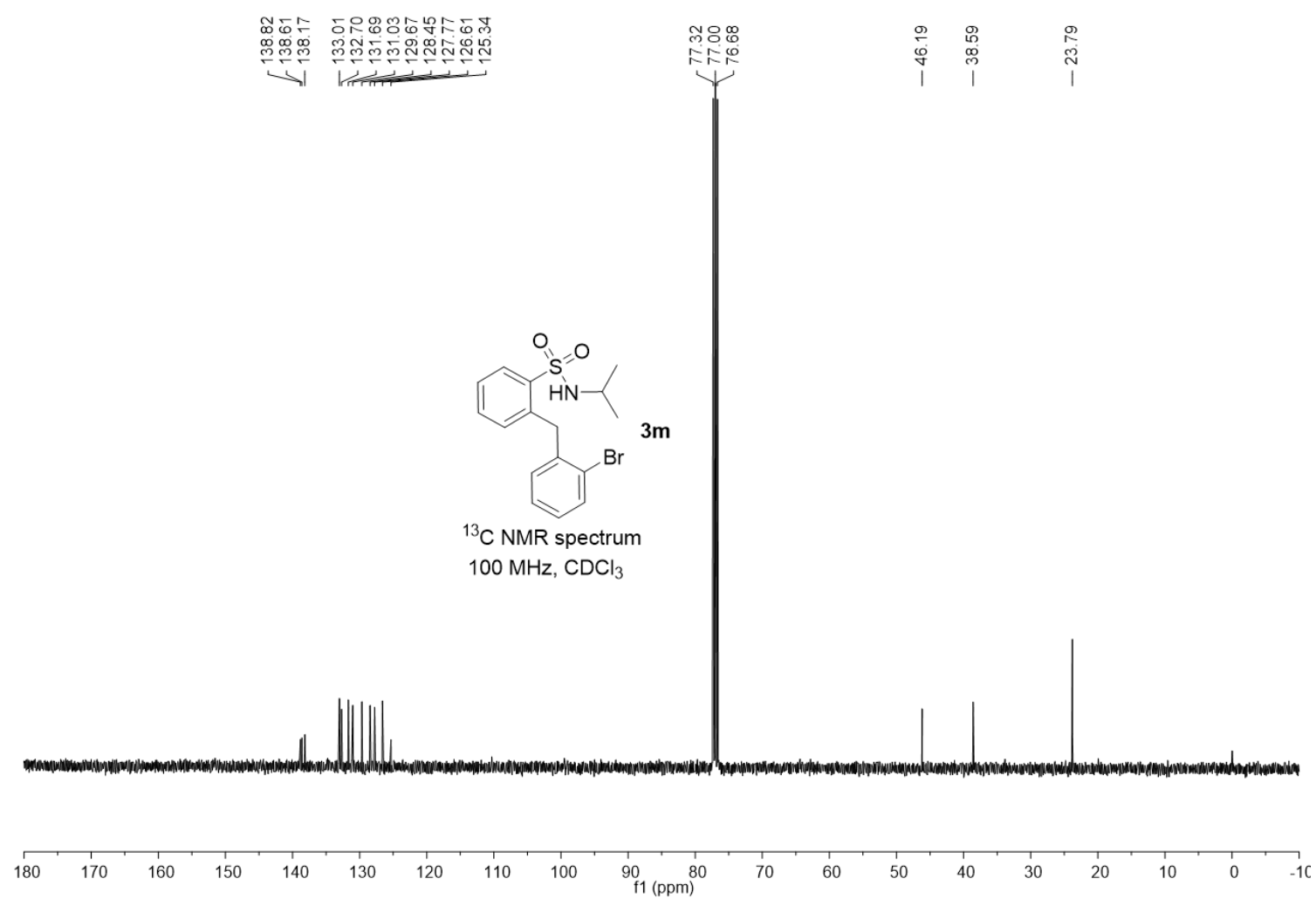

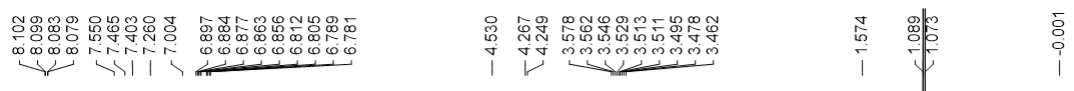

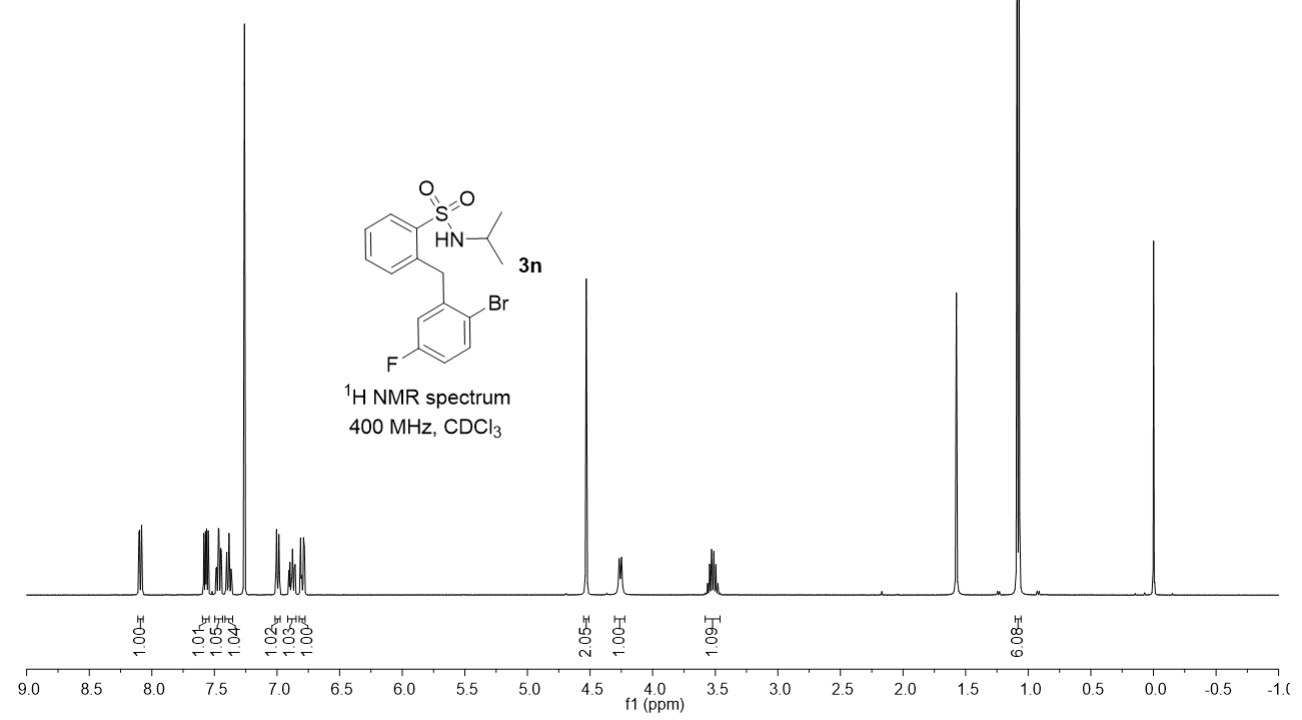



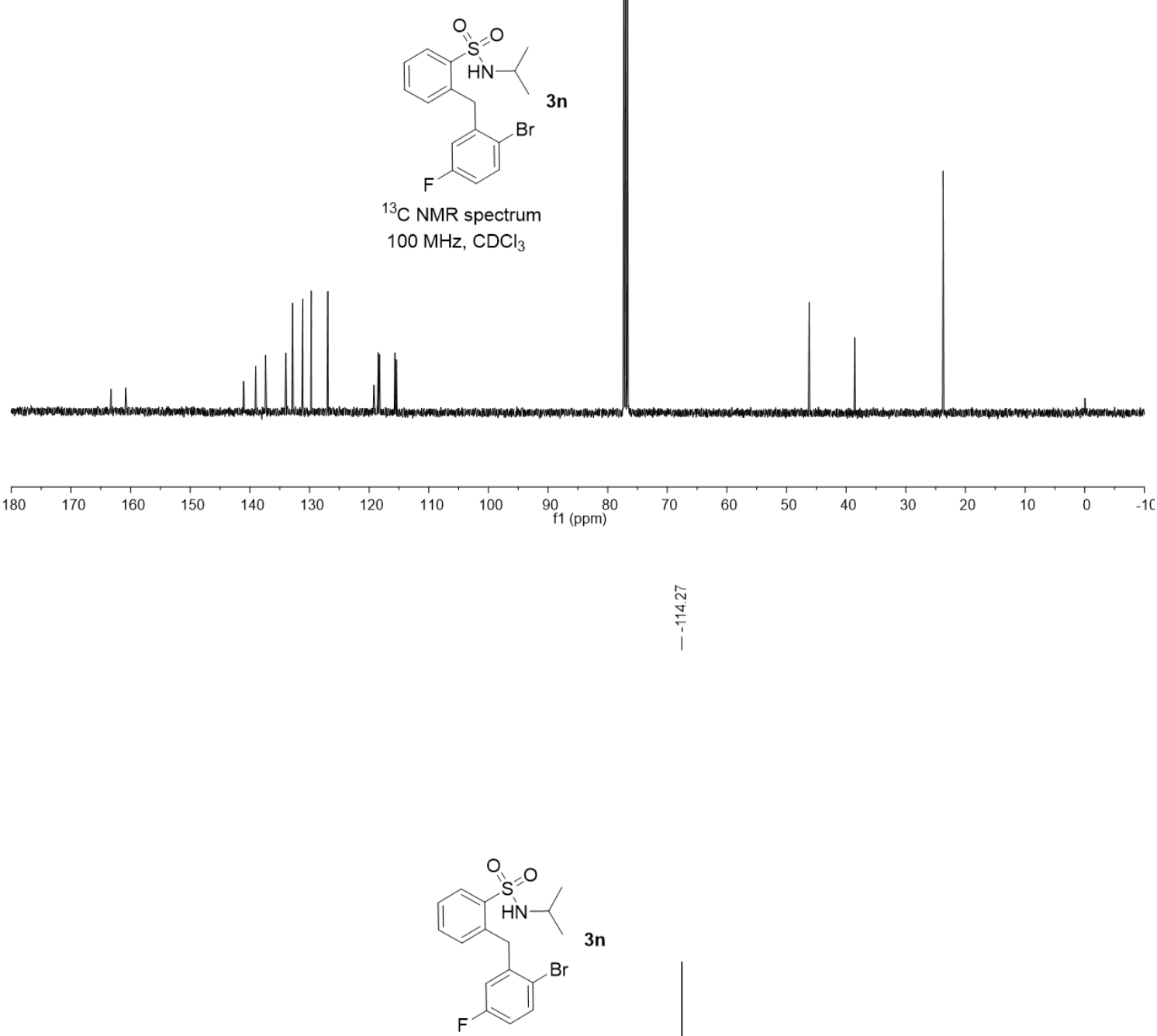

${ }^{19} \mathrm{~F}$ NMR spectrum

$375 \mathrm{MHz}, \mathrm{CDCl}_{3}$

\begin{tabular}{rllllllllllllllllllllll}
\hline & 10 & 0 & -10 & -20 & -30 & -40 & -50 & -60 & -70 & -80 & -90 & -100 & -110 & -120 & -130 & -140 & -150 & -160 & -170 & -180 & -190 & -20
\end{tabular} 


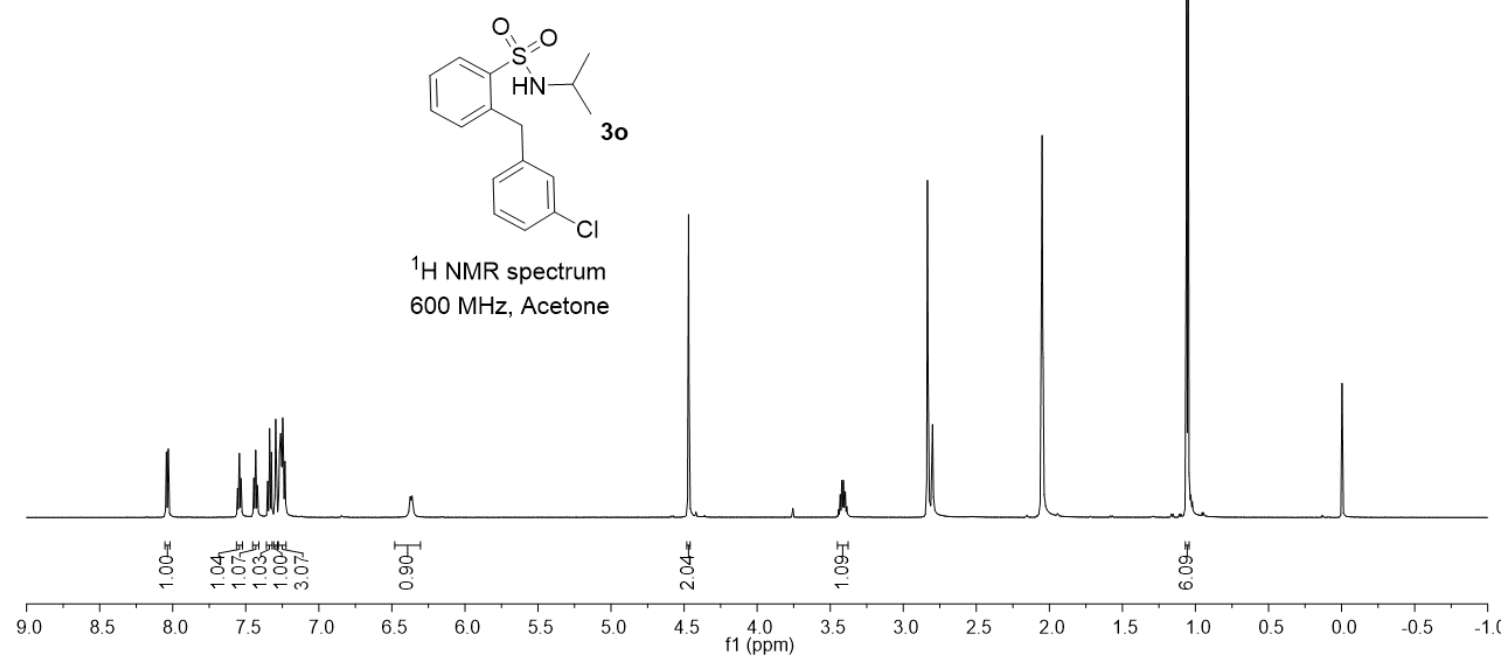

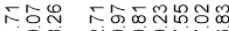

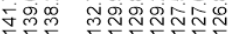

i.

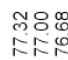

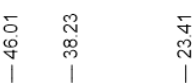

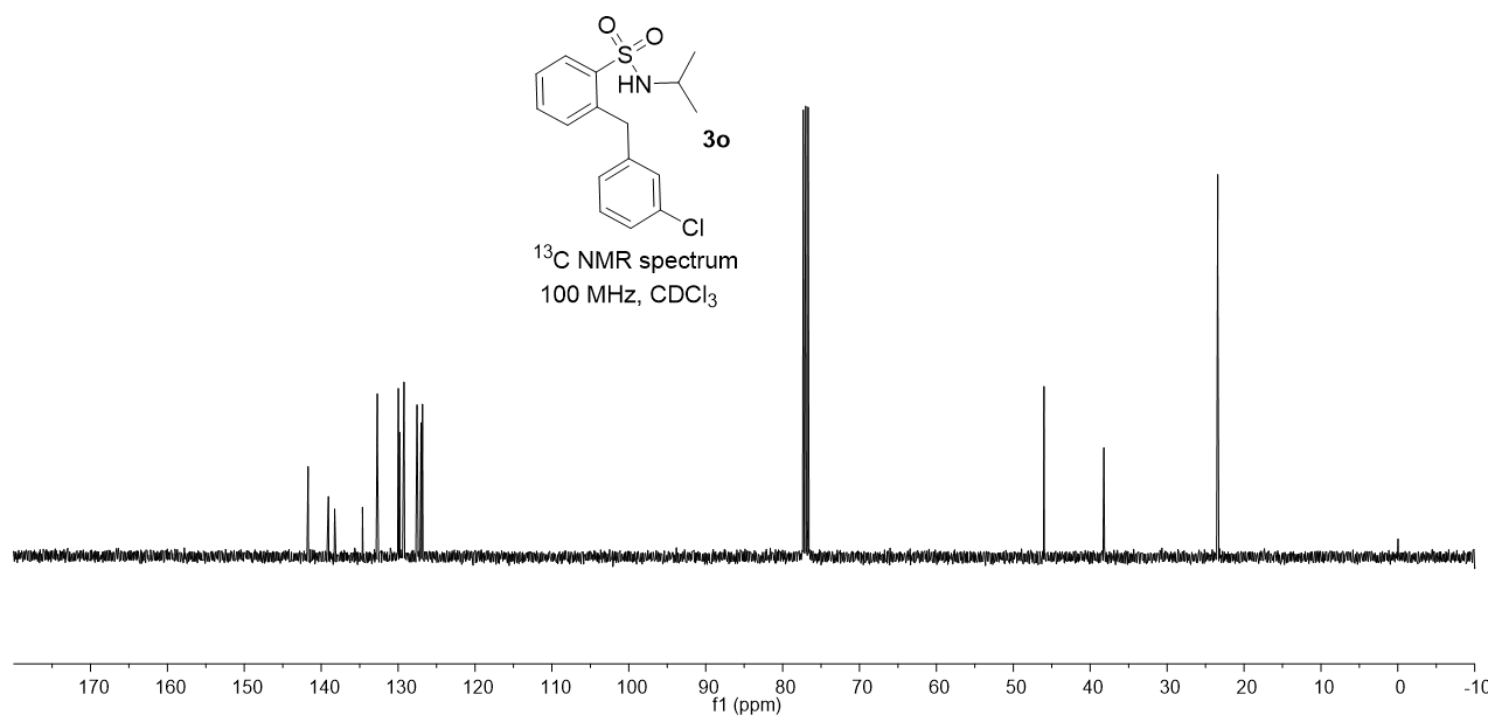



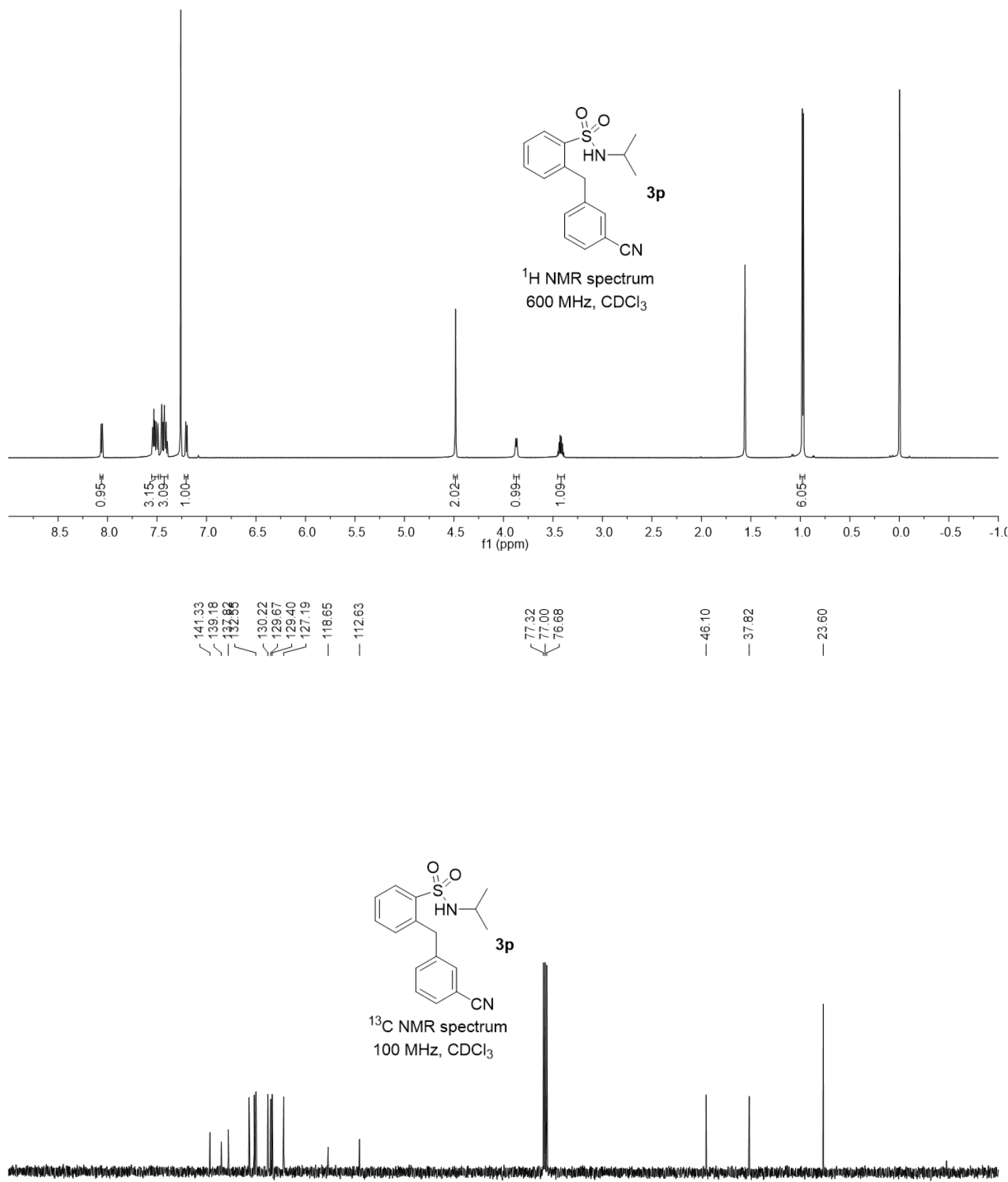

$\begin{array}{lllllllllll}180 & 170 & 160 & 150 & 140 & 130 & 120 & 110 & 100 & 90 & 1\end{array}$ 

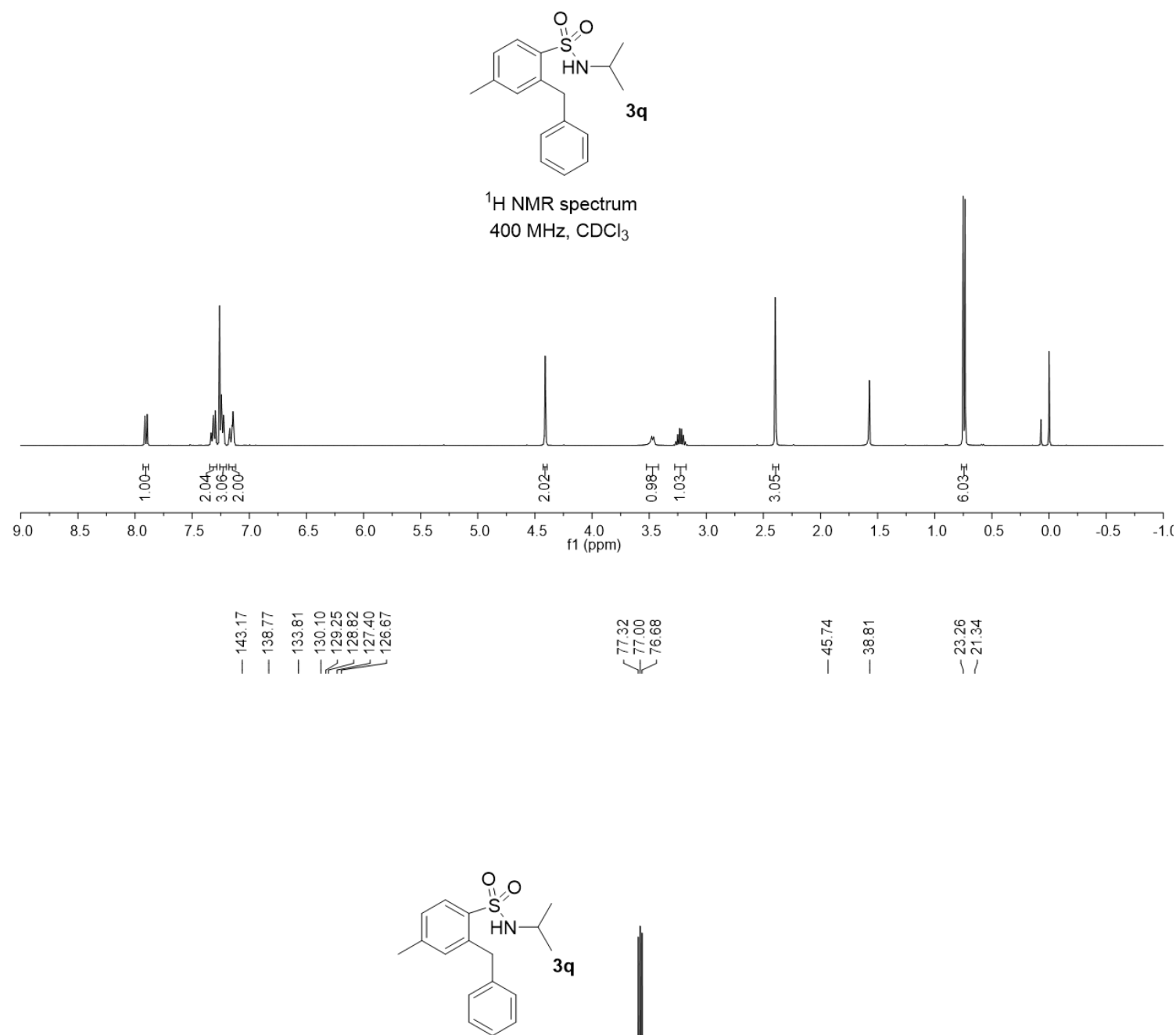

${ }^{13} \mathrm{C}$ NMR spectrum $100 \mathrm{MHz}_{\mathrm{CDCl}}$ 


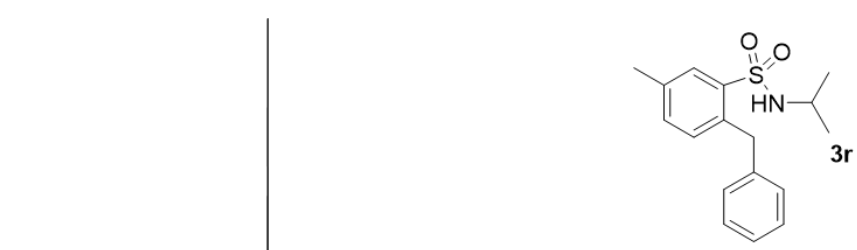

H NMR spectrum $400 \mathrm{MHz} \mathrm{CDCl}_{3}$

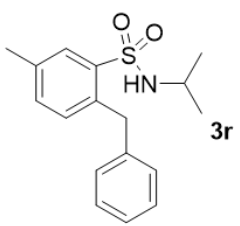

${ }^{13} \mathrm{C}$ NMR spectrum $100 \mathrm{MHz}, \mathrm{CDCl}_{3}$

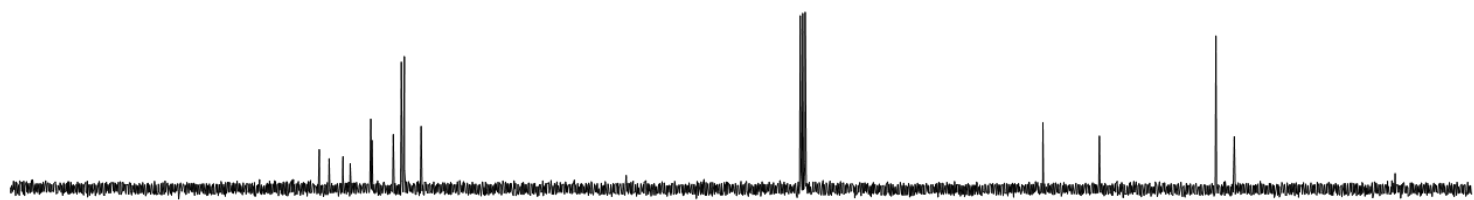

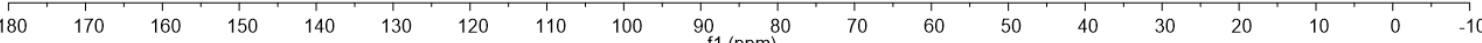




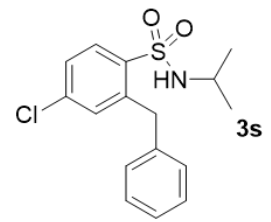

${ }^{1} \mathrm{H}$ NMR spectrum

$400 \mathrm{MHz}, \mathrm{CDCl}_{3}$

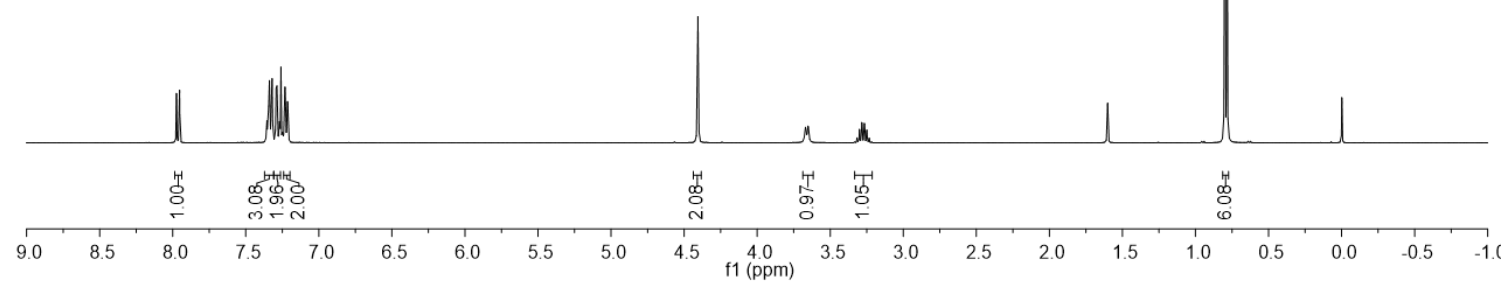

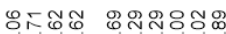

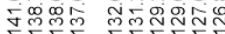

స్ㅇำ

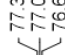

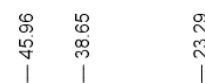

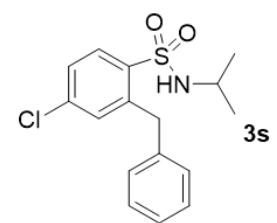

${ }^{13} \mathrm{C}$ NMR spectrum

$100 \mathrm{MHz}, \mathrm{CDCl}_{3}$

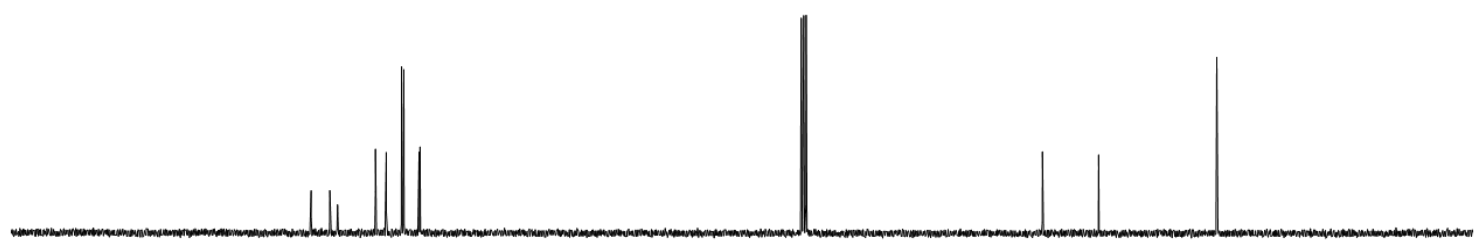

\begin{tabular}{rl|llllllllllllllllllll}
\hline 180 & 170 & 160 & 150 & 140 & 130 & 120 & 110 & 100 & 90 & 80 & 70 & 60 & 50 & 40 & 30 & 20 & 10 & 0 & -10
\end{tabular} 


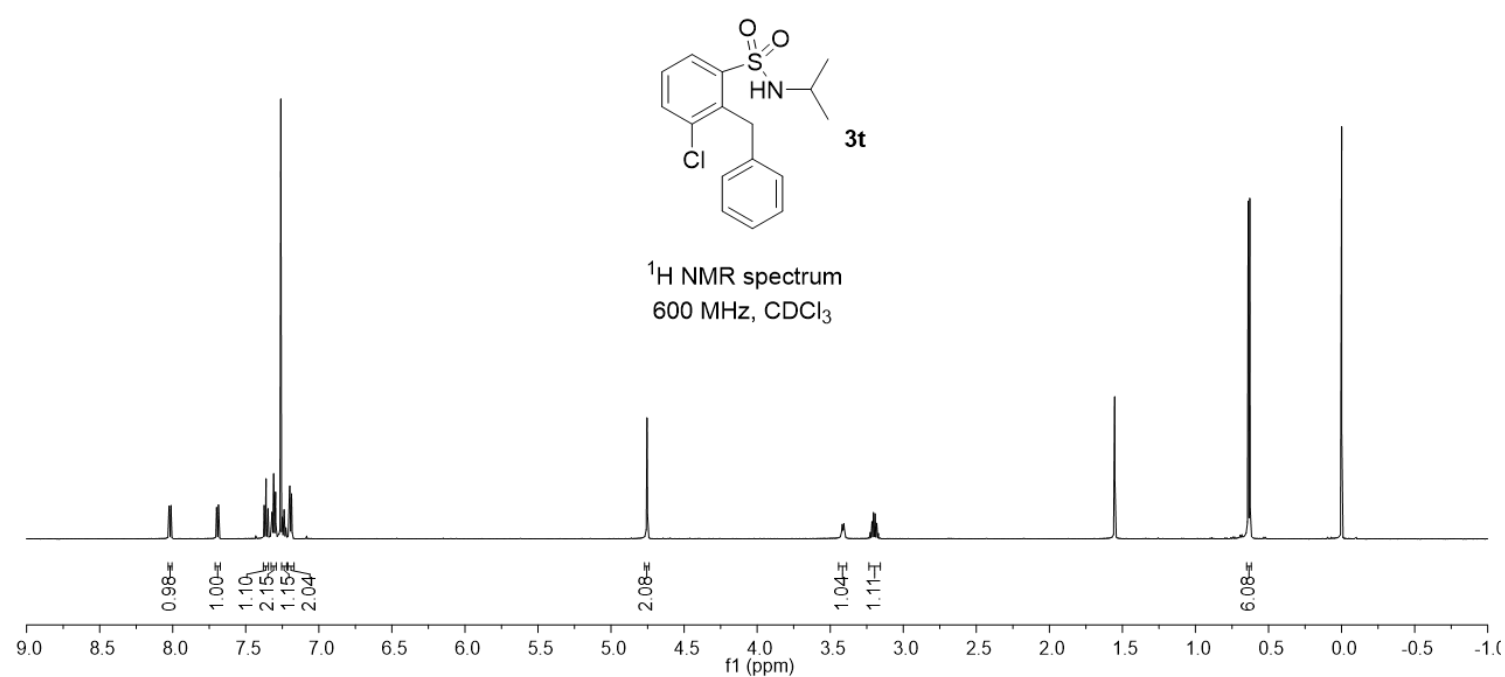

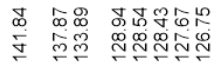

i

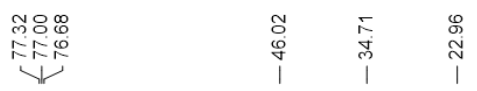

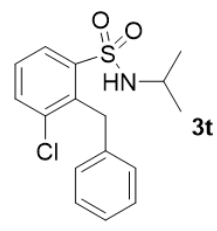

${ }^{13} \mathrm{C}$ NMR spectrum

$100 \mathrm{MHz}, \mathrm{CDCl}_{3}$

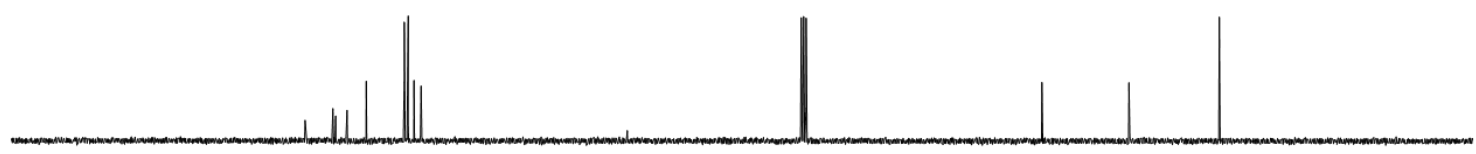

180

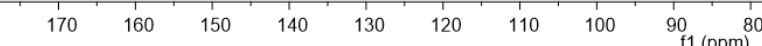



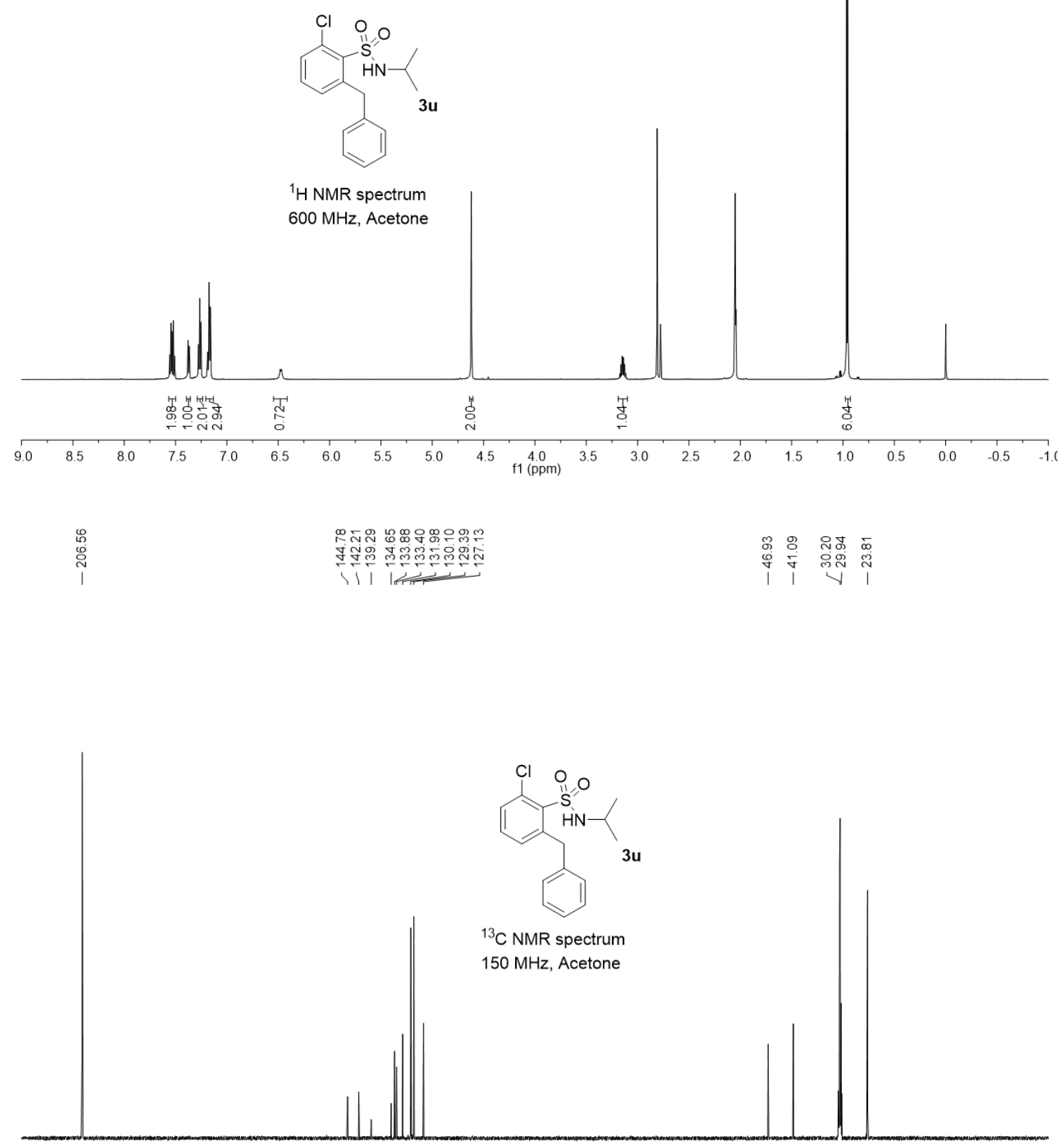

$\begin{array}{llllllllllllllllllllllllllll}1 & 220 & 210 & 200 & 190 & 180 & 170 & 160 & 150 & 140 & 130 & 120 & 110 & 100 & 90 & 80 & 70 & 60 & 50 & 40 & 30 & 20 & 10 & 0 & -10\end{array}$ 

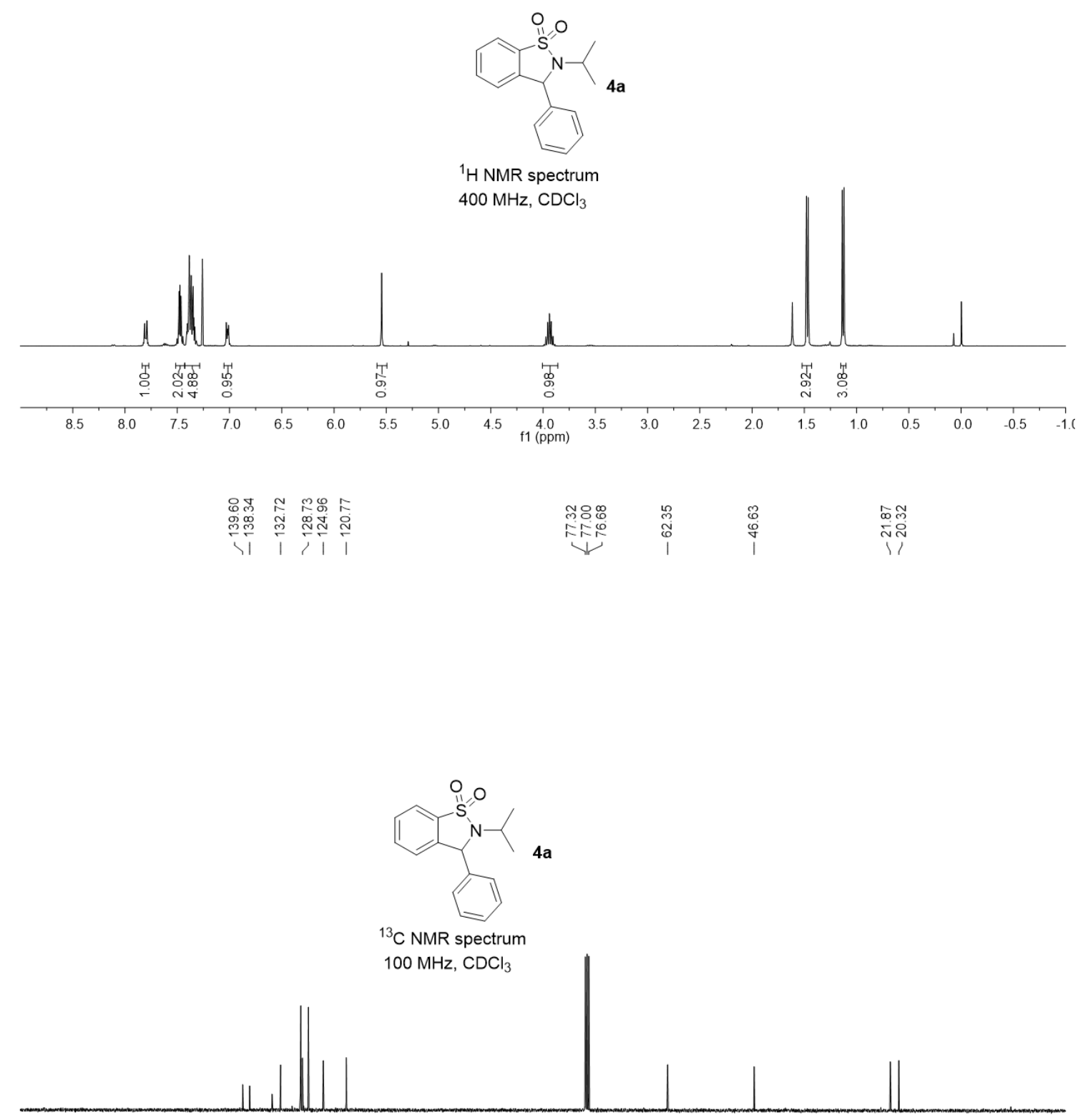

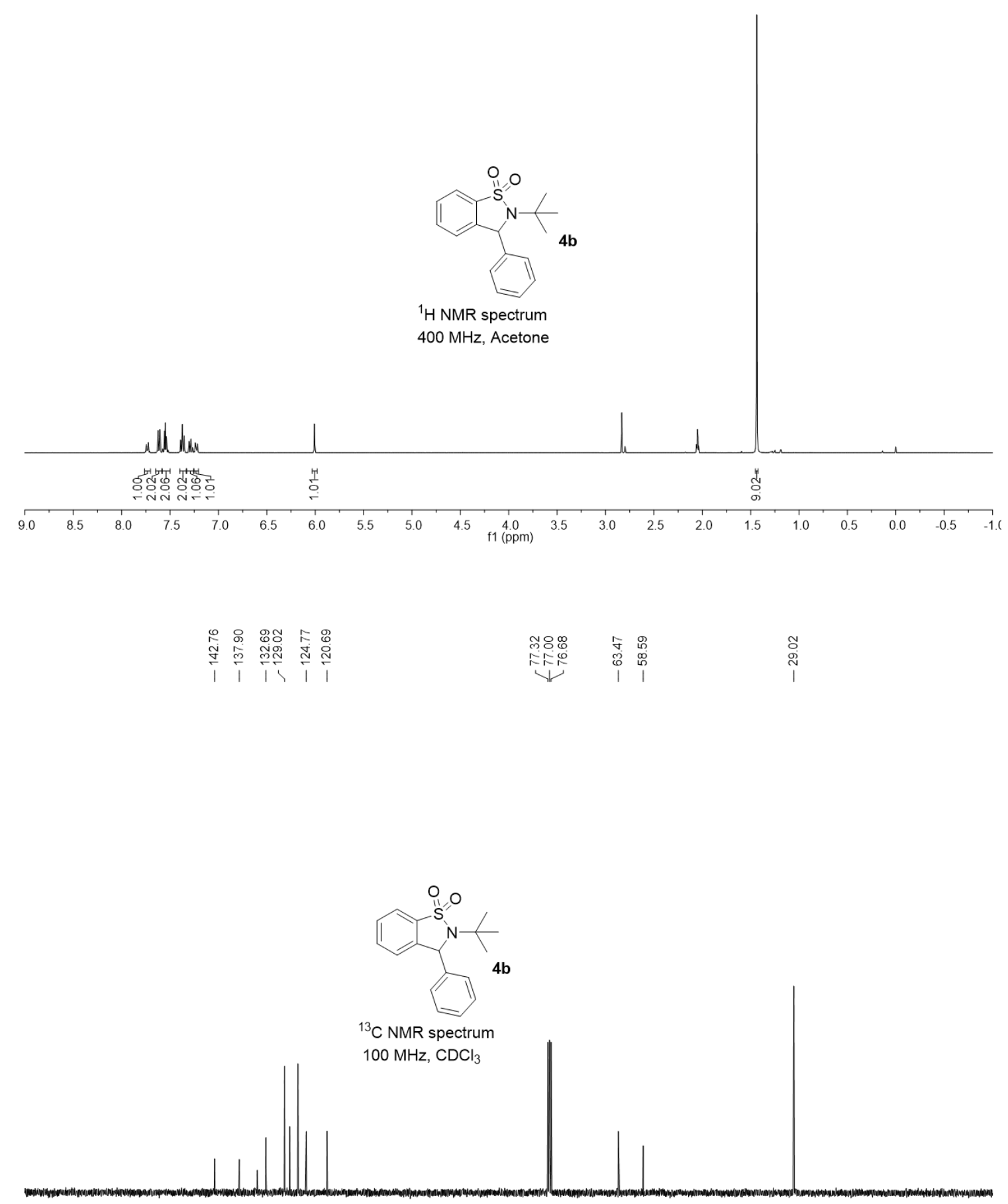

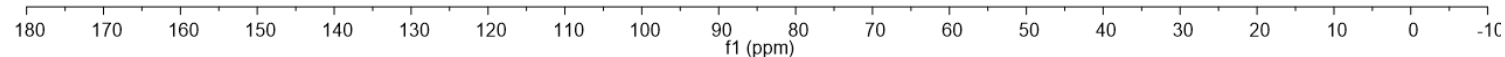




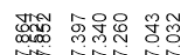

niminin

$\underset{\text { in }}{\stackrel{0}{\infty}}$

$\stackrel{\substack{R \\ N}}{\substack{\infty \\ i}}$

@o

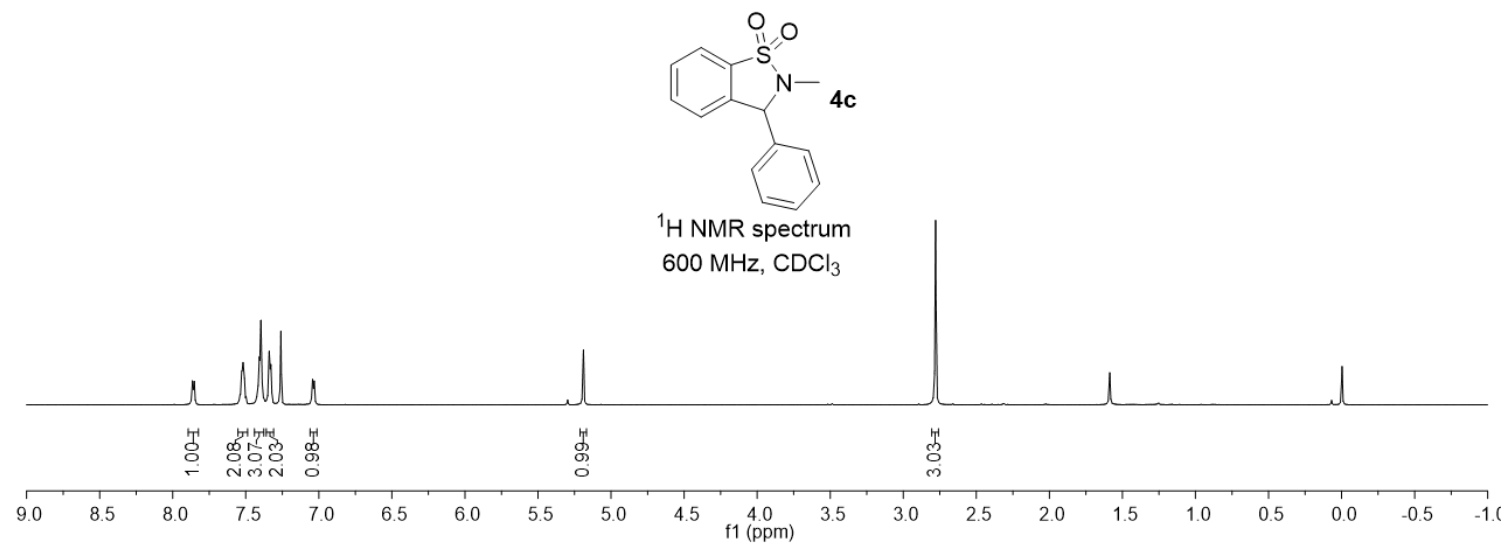

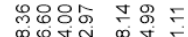

mల్లాల్ల

परिए।

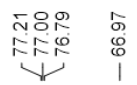

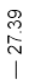

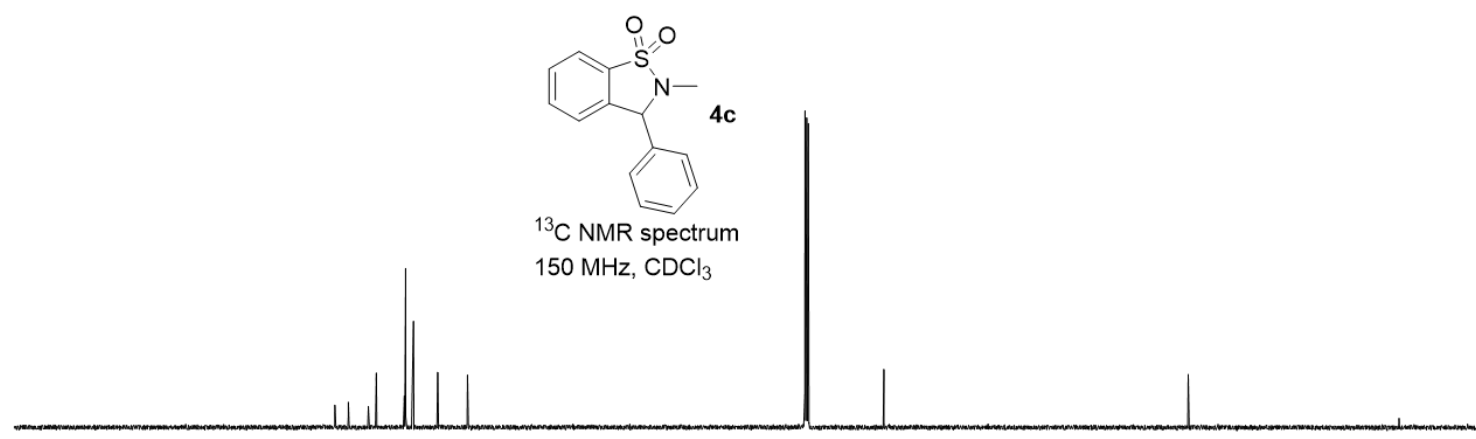

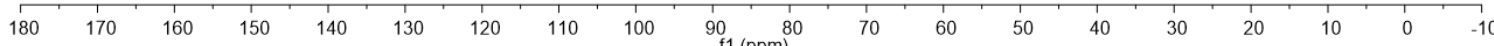




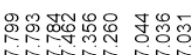

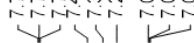

$\underset{\substack{\text { N } \\ \text { I } \\ \text { I }}}{1}$

急

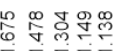

江

@ั

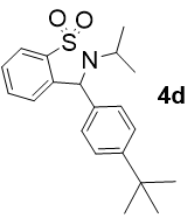

${ }^{1} \mathrm{H}$ NMR spectrum

$600 \mathrm{MHz}, \mathrm{CDCl}_{3}$

4d

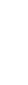
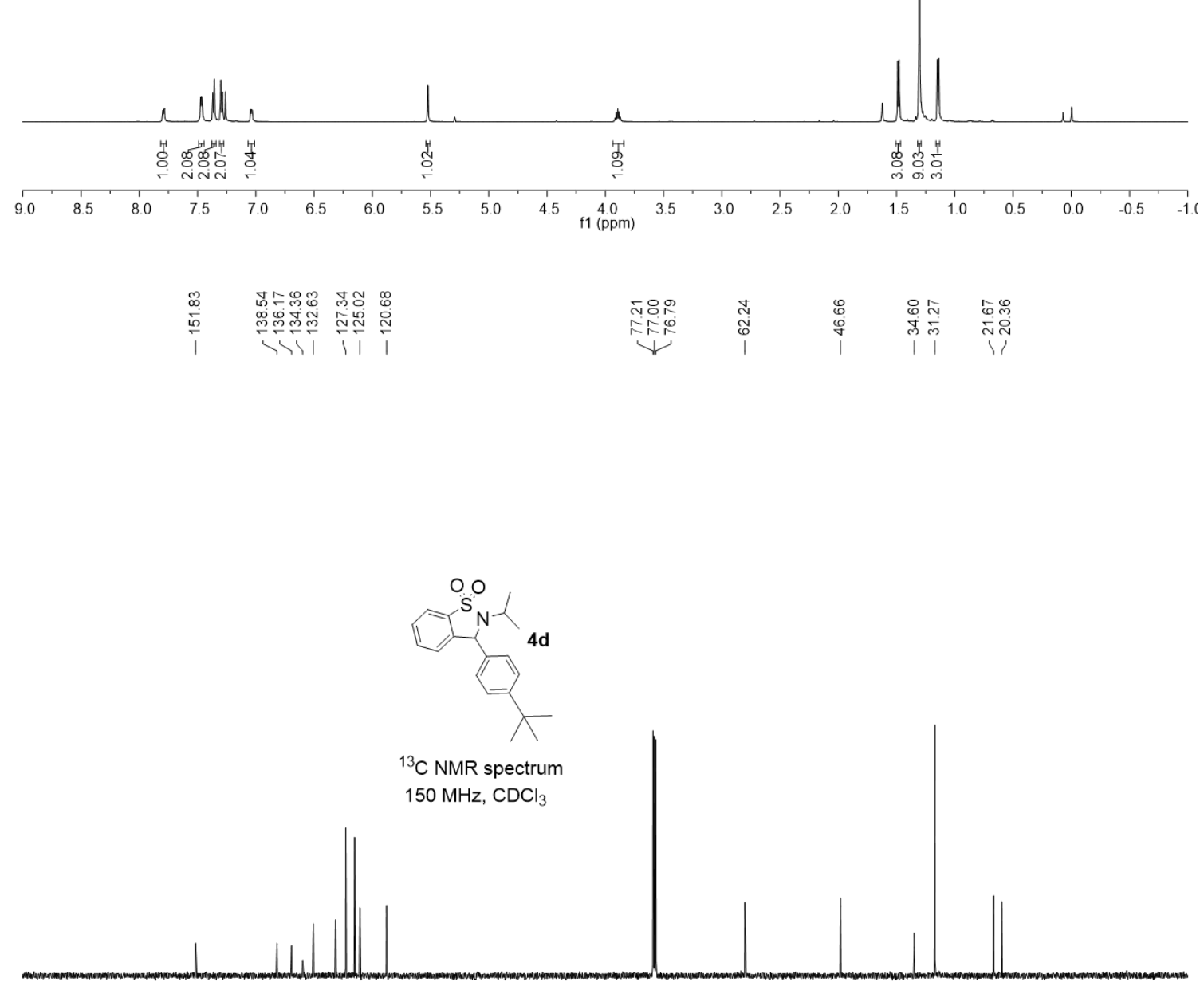

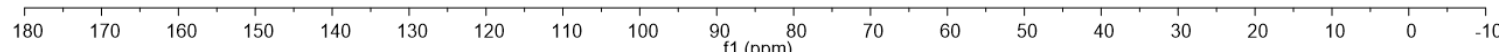




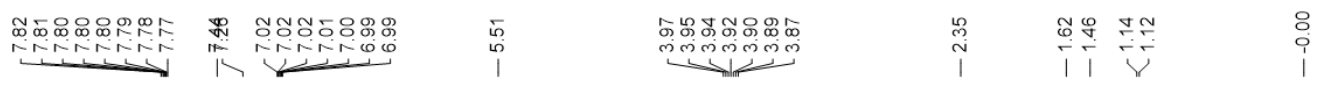

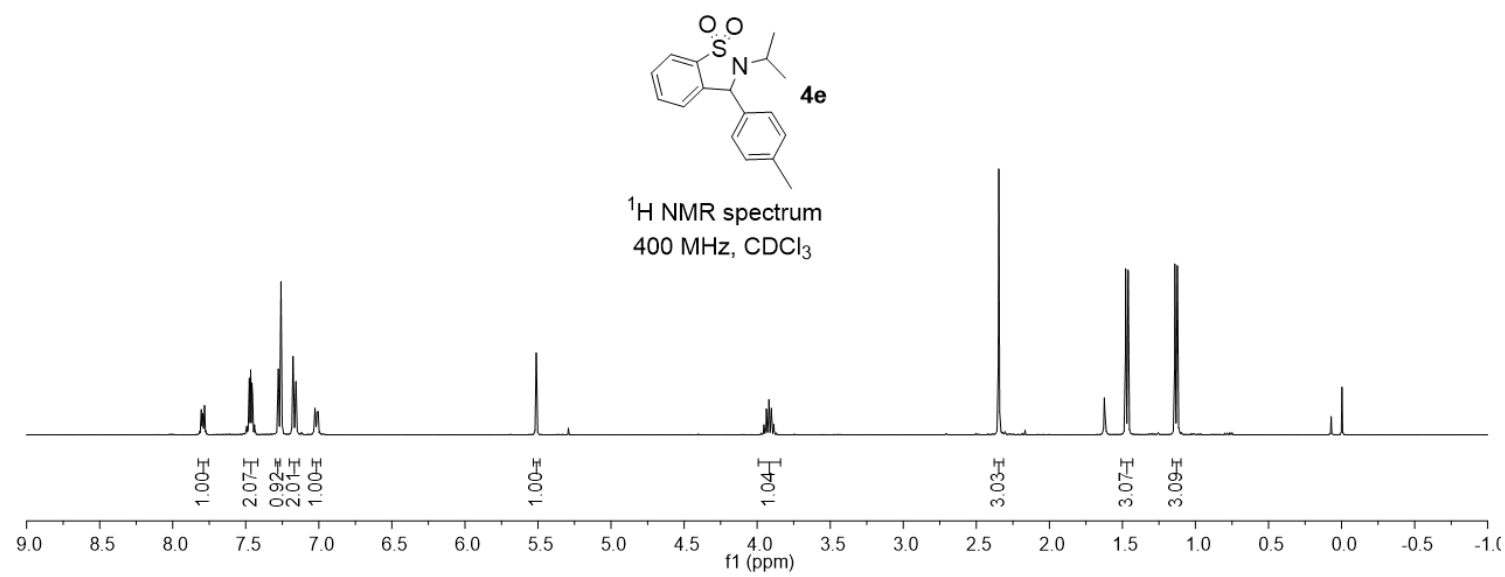

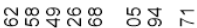

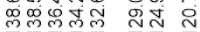

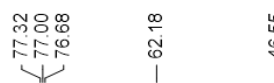

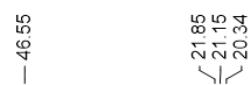

们;

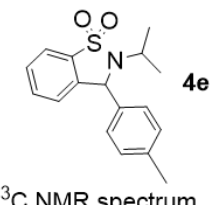

${ }^{13} \mathrm{C}$ NMR spectrum

$100 \mathrm{MHz} \mathrm{CDCl}_{3}$

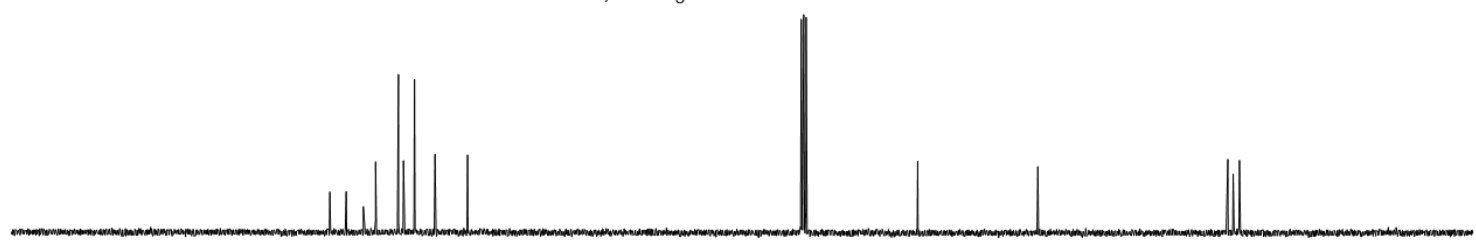

180

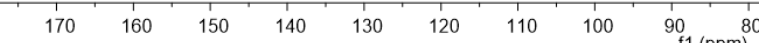




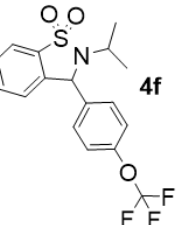

${ }^{1} \mathrm{H}$ NMR spectrum

$400 \mathrm{MHz}, \mathrm{CDCl}_{3}$
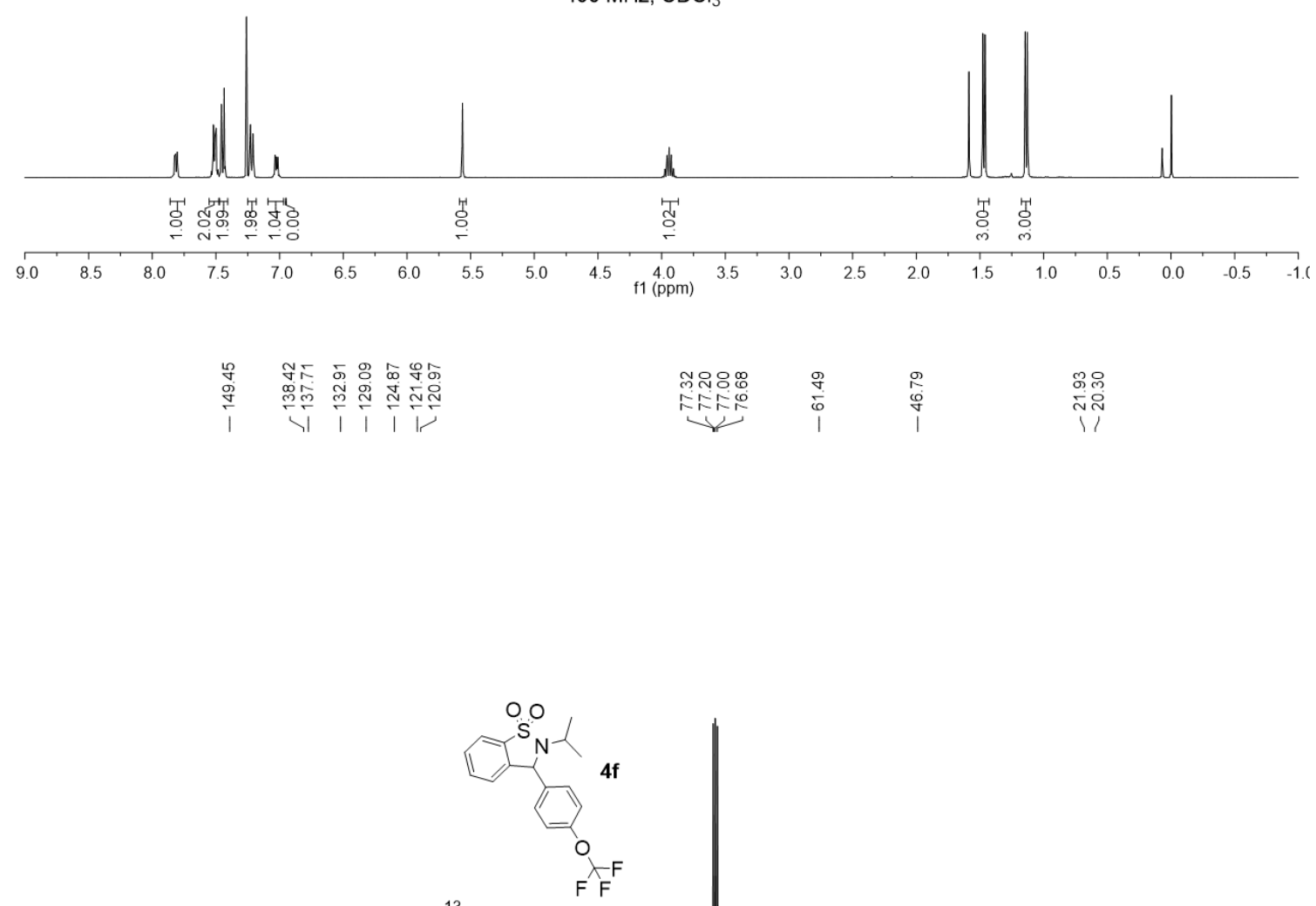

${ }^{13} \mathrm{C}$ NMR spectrum

$100 \mathrm{MHz}, \mathrm{CDCl}_{3}$

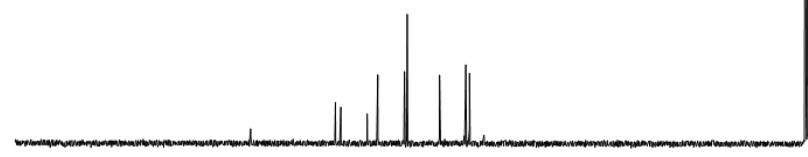

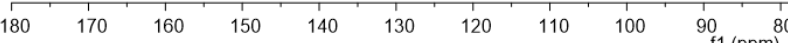




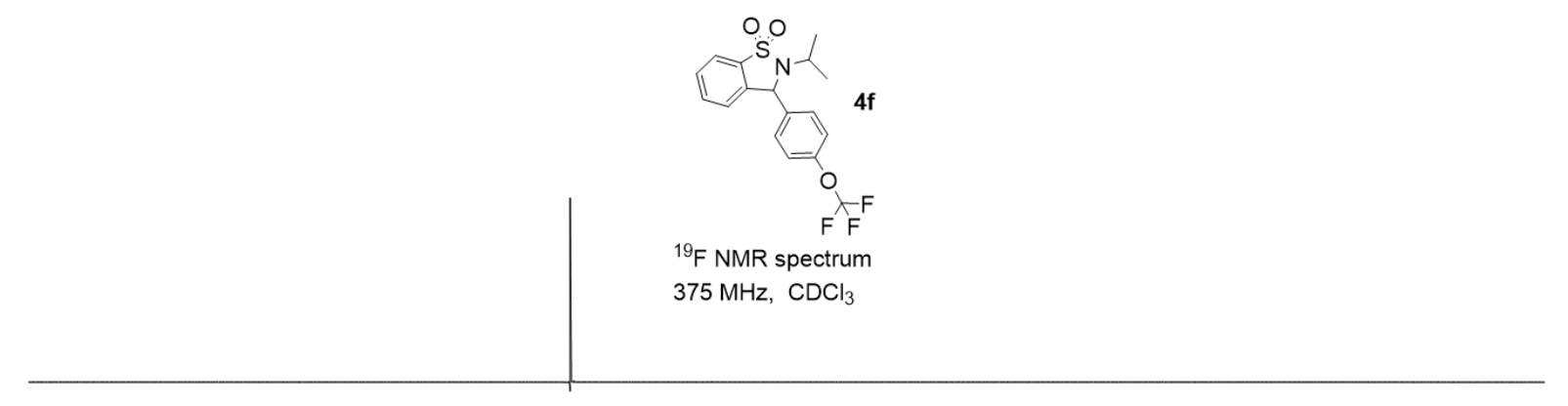

\begin{tabular}{|c|c|c|c|c|c|c|c|c|c|c|c|c|c|c|c|c|c|c|c|}
\hline 10 & 0 & -10 & -20 & -30 & -40 & -50 & -60 & -70 & $\begin{array}{c}-80 \\
\mathrm{f} 1\end{array}$ & m) & -100 & -110 & -120 & -130 & -140 & -150 & -160 & -170 & -18 \\
\hline
\end{tabular}

\begin{tabular}{|c|c|c|c|c|c|c|c|}
\hline 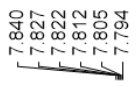 & 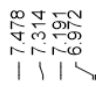 & 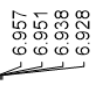 & $\underset{\substack { N \\
\infty \\
\infty \\
\begin{subarray}{c}{N \\
1{ N \\
\infty \\
\infty \\
\begin{subarray} { c } { N \\
1 } }\end{subarray}}{1}$ & 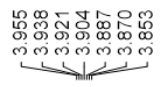 & $\stackrel{\substack{\text { N } \\
\text { I }}}{1}$ & $\underbrace{-10}$ & $\frac{N ָ}{\stackrel{N}{\leftarrow}}$ \\
\hline
\end{tabular}

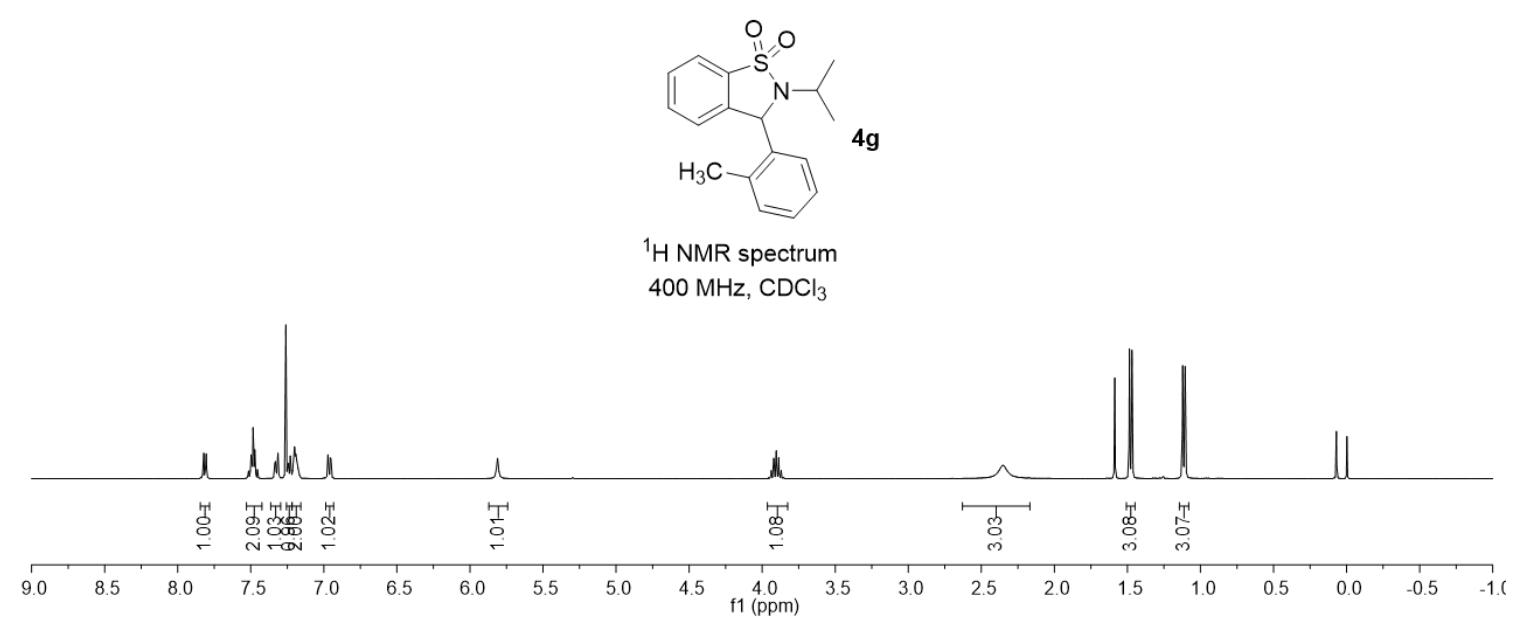



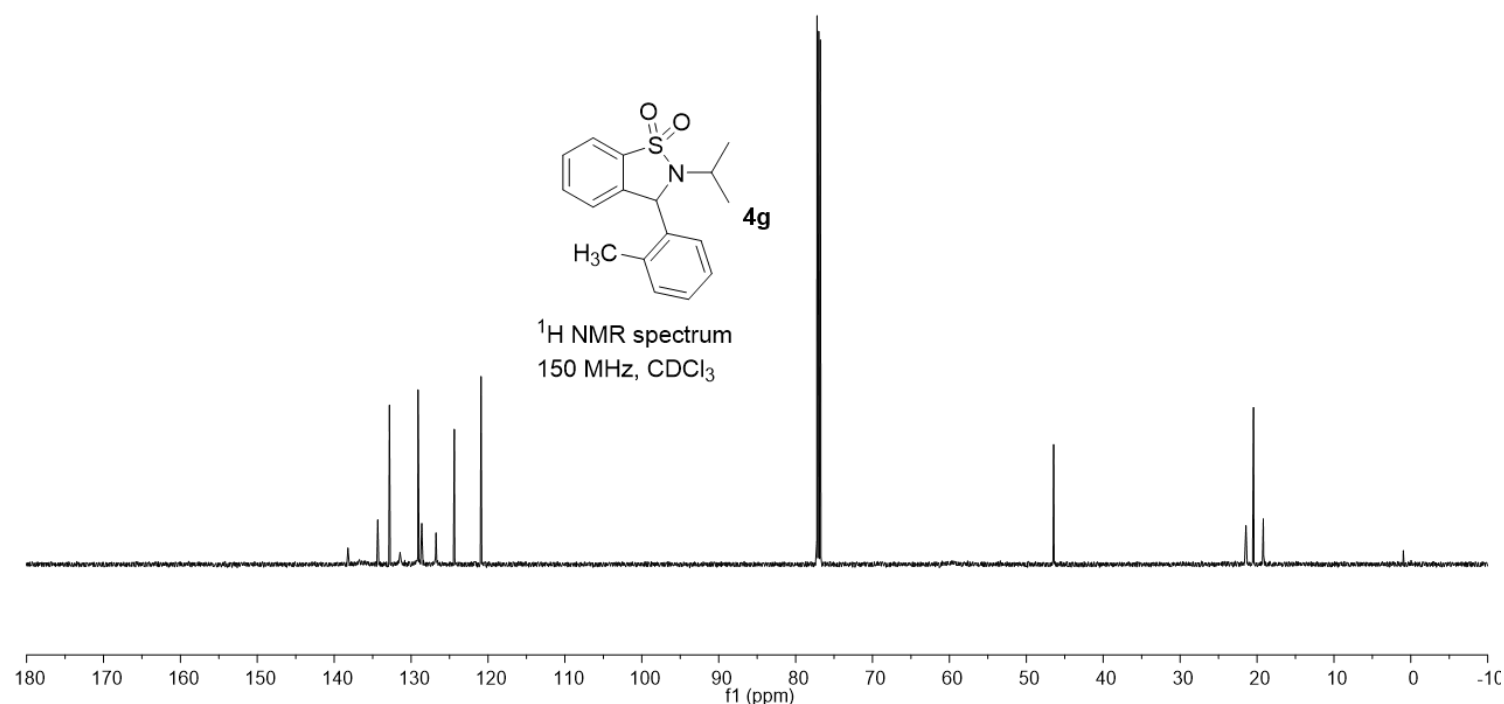

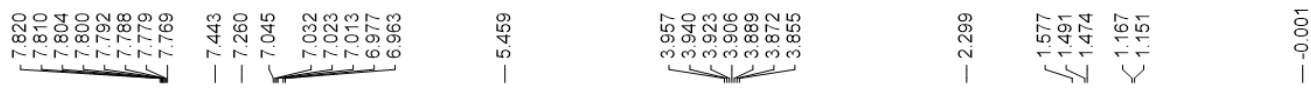

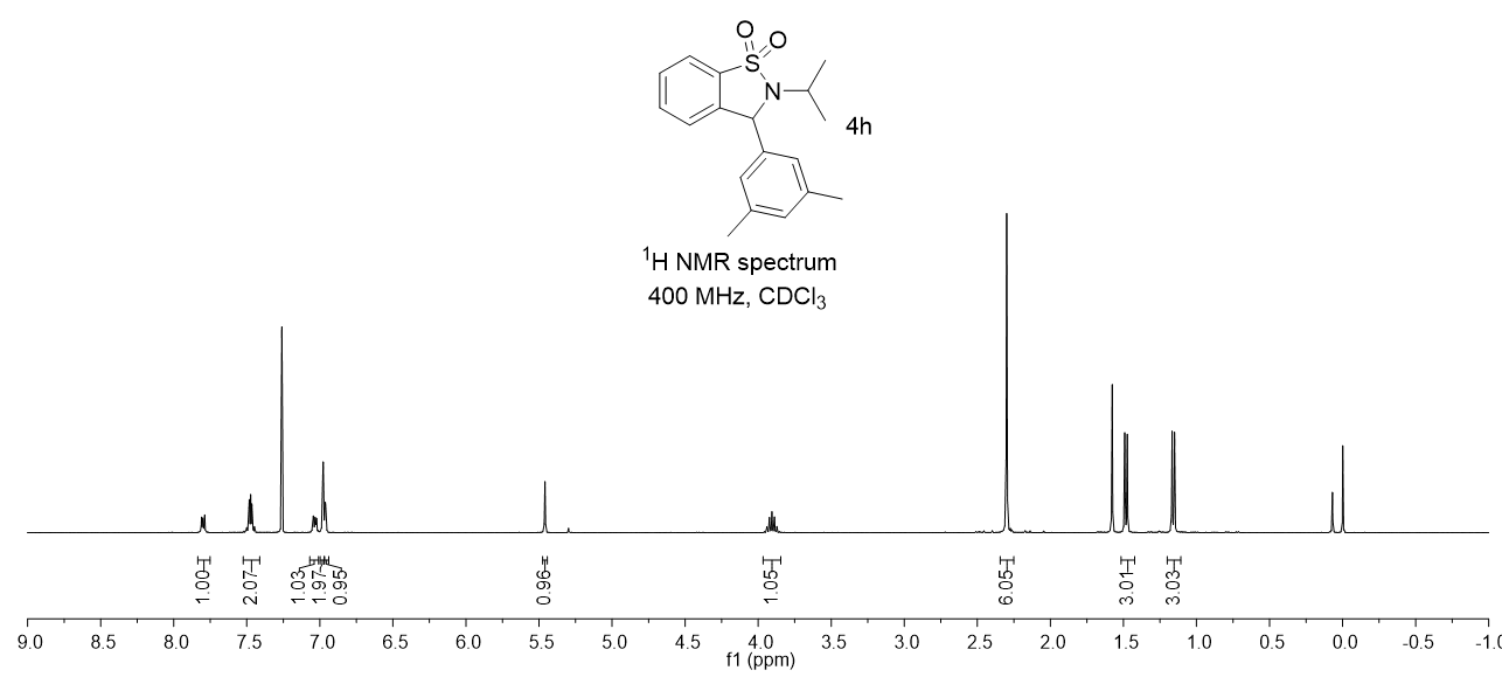




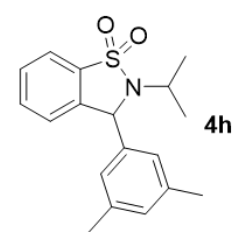

$4 h$

${ }^{13} \mathrm{C}$ NMR spectrum

$100 \mathrm{MHz}, \mathrm{CDCl}_{3}$
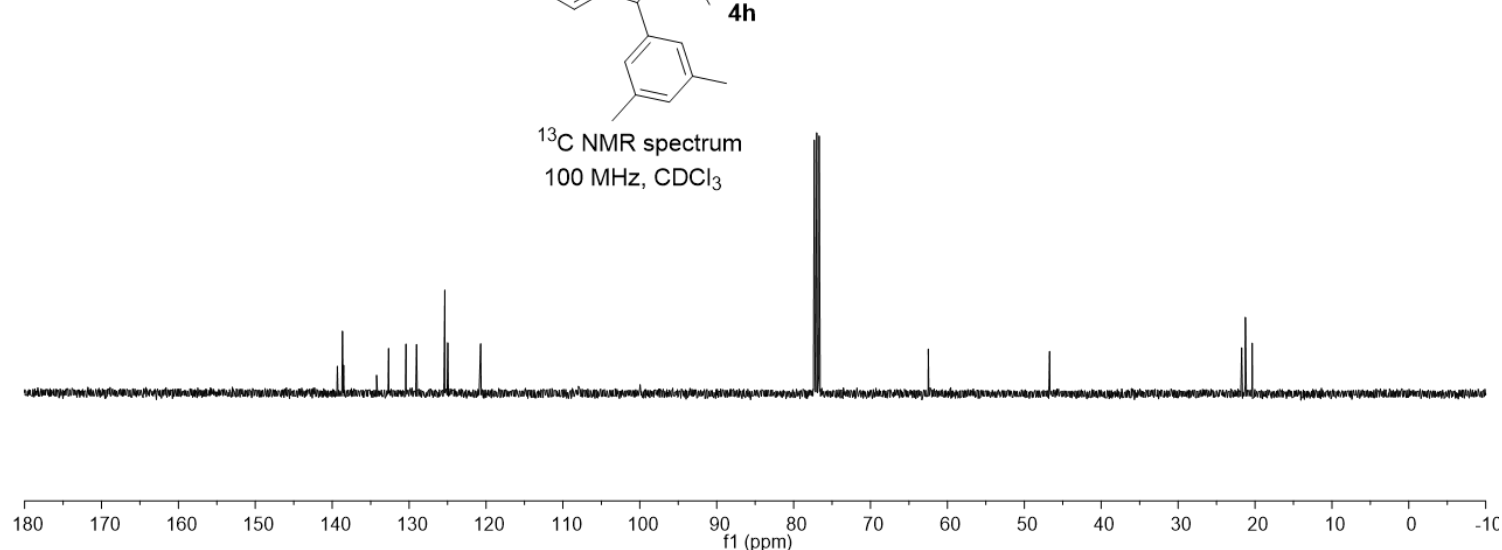

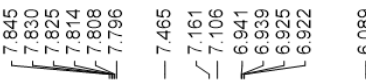

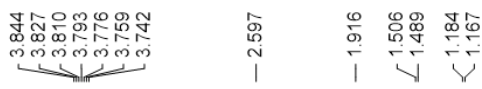

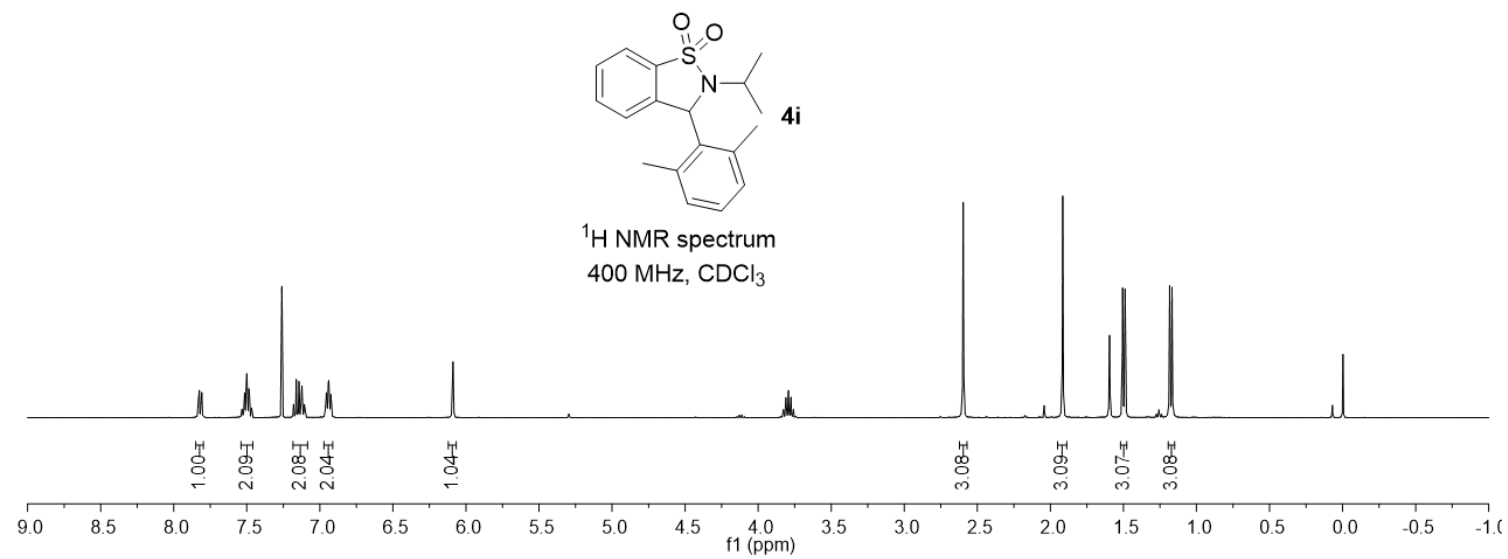




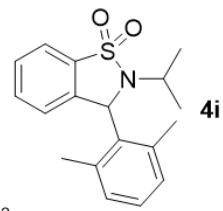

$4 i$

${ }^{13} \mathrm{C}$ NMR spectrum

$100 \mathrm{MHz} \mathrm{CDCl}_{3}$
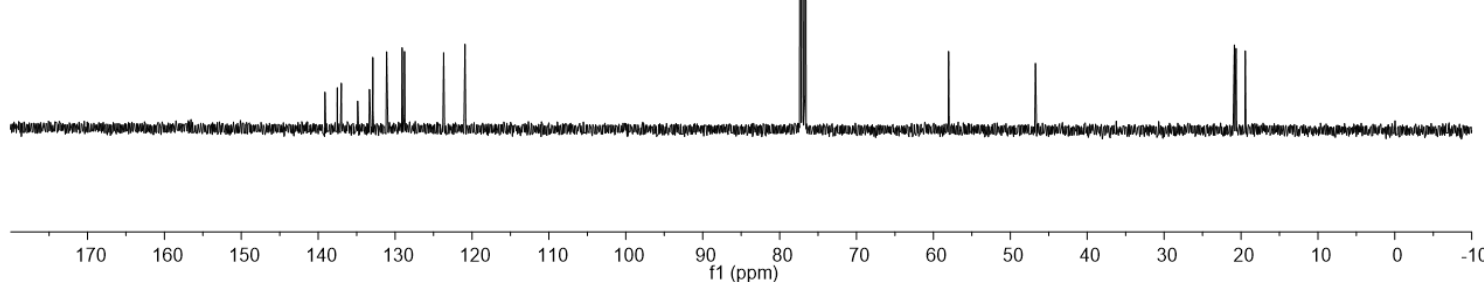

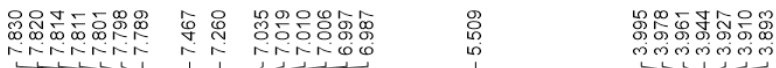

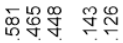

车

0
8
0
1

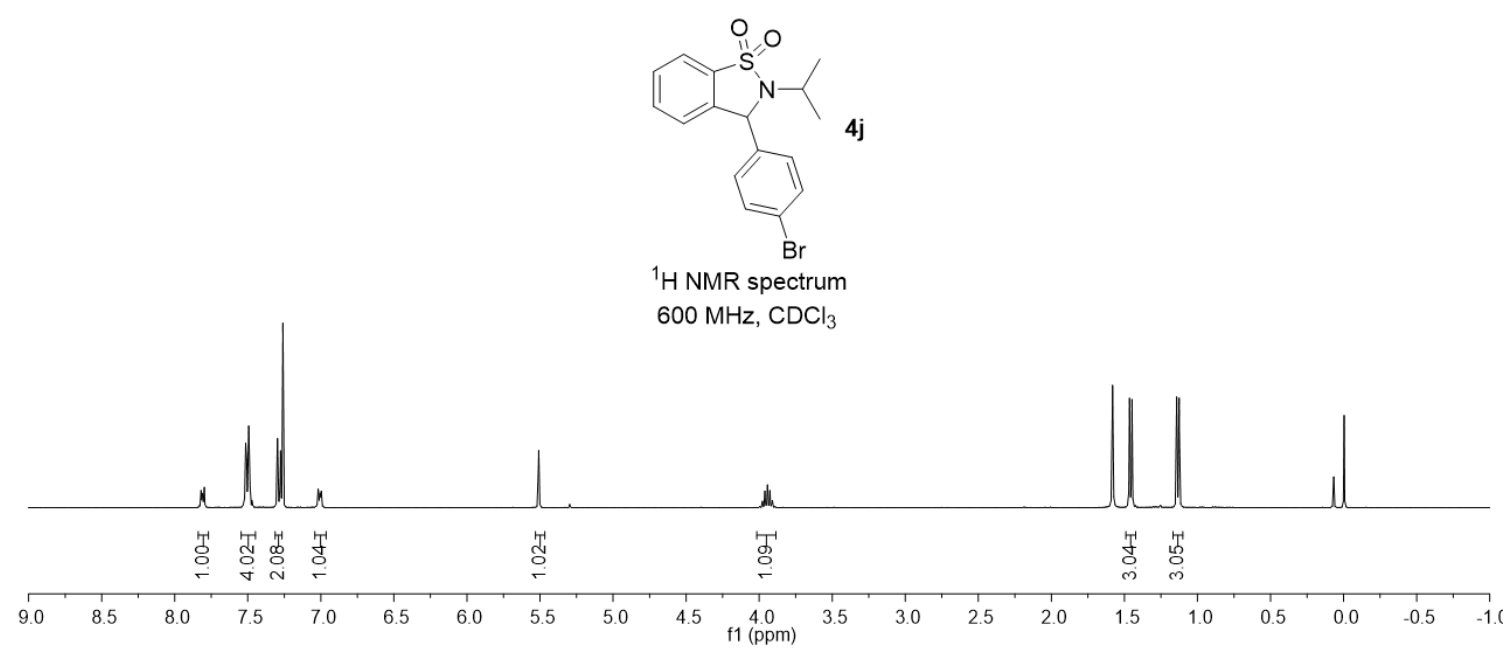




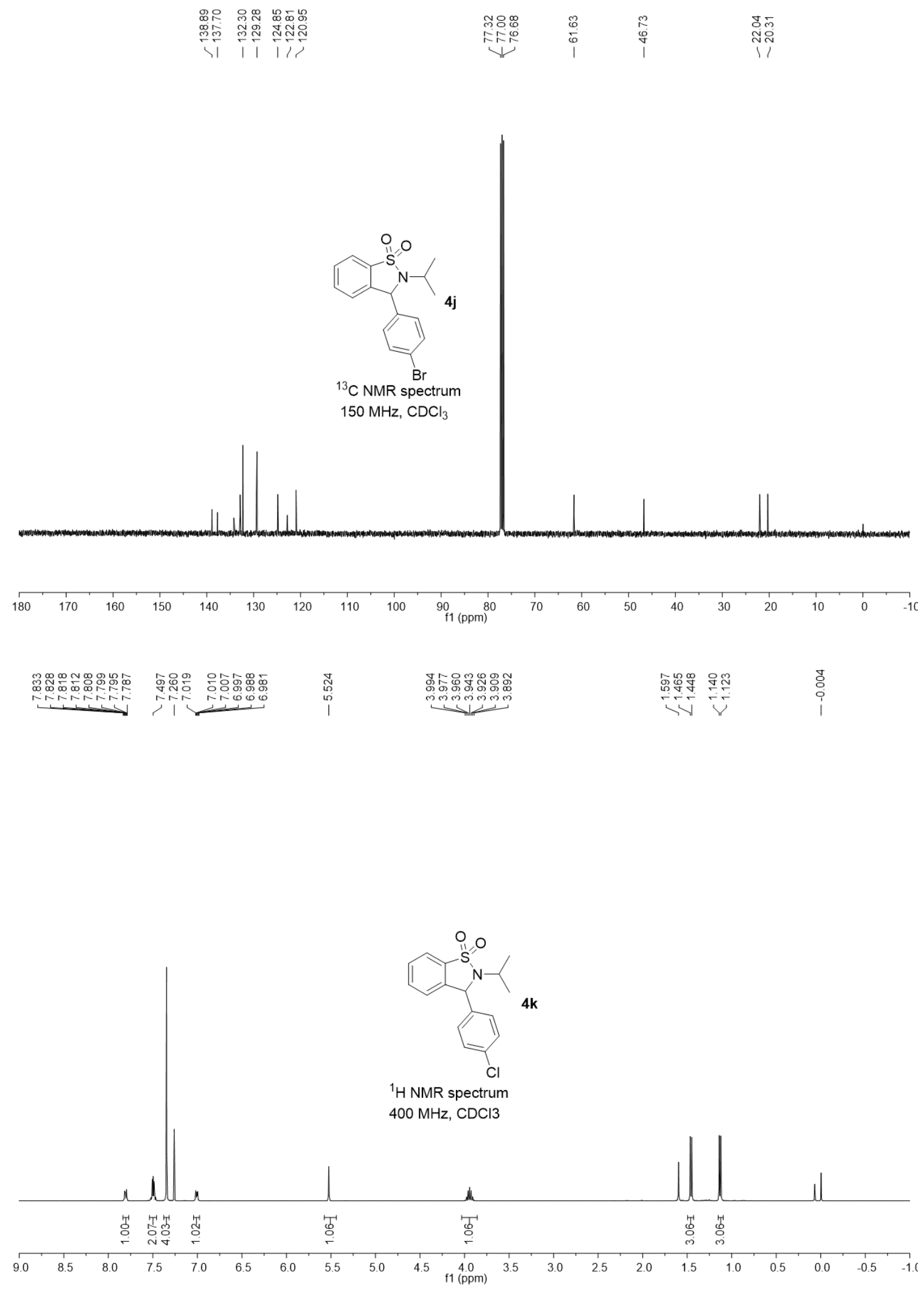




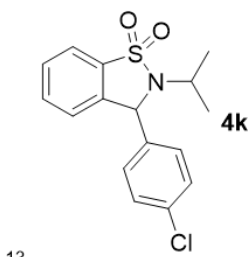

${ }^{13} \mathrm{C}$ NMR spectrum $100 \mathrm{MHz} \mathrm{CDCl}_{3}$
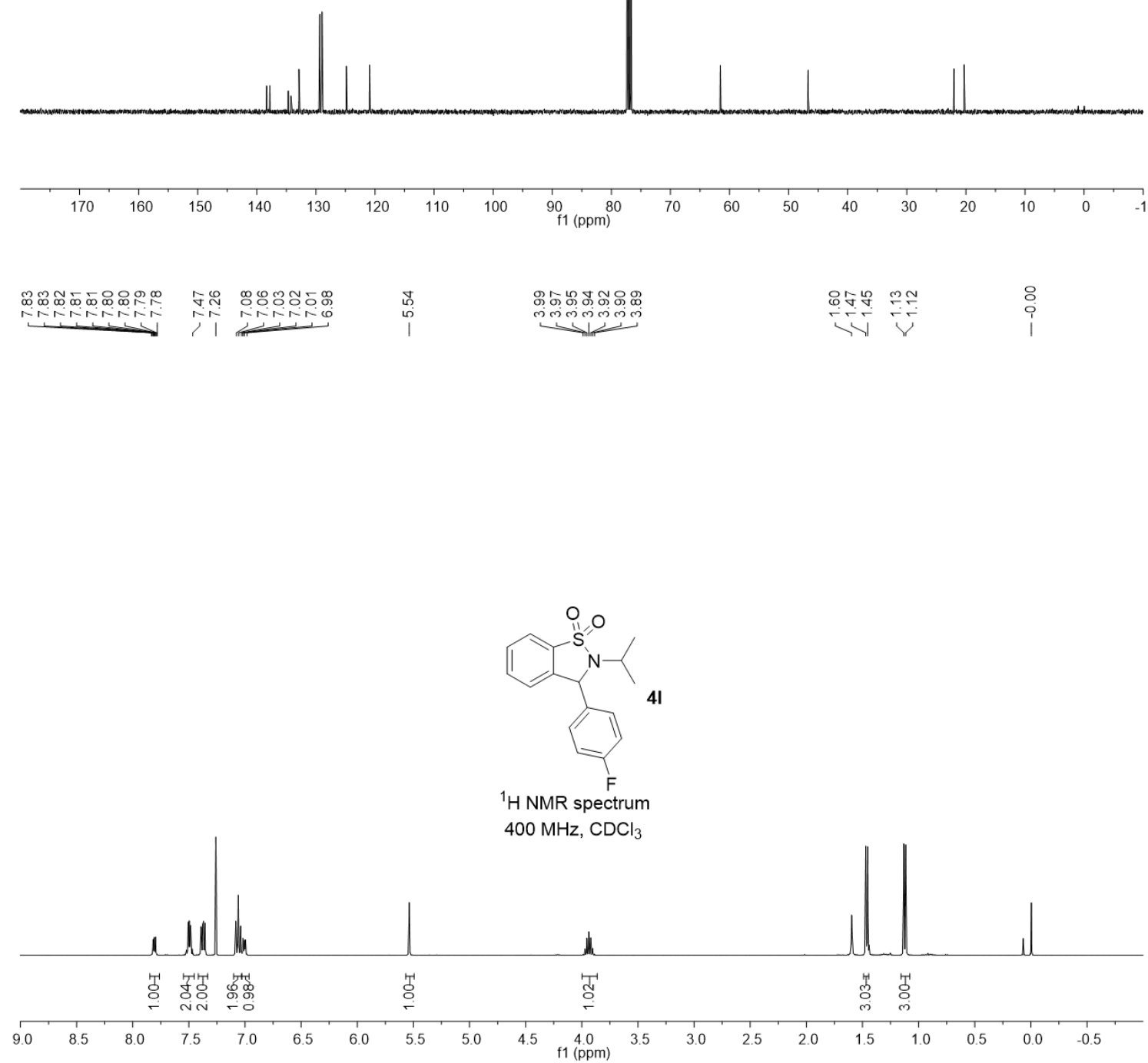


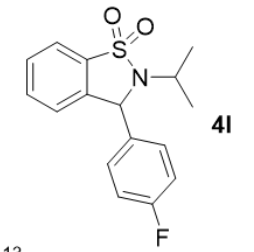

${ }^{13} \mathrm{C}$ NMR spectrum

$100 \mathrm{MHz}, \mathrm{CDCl}_{3}$

41
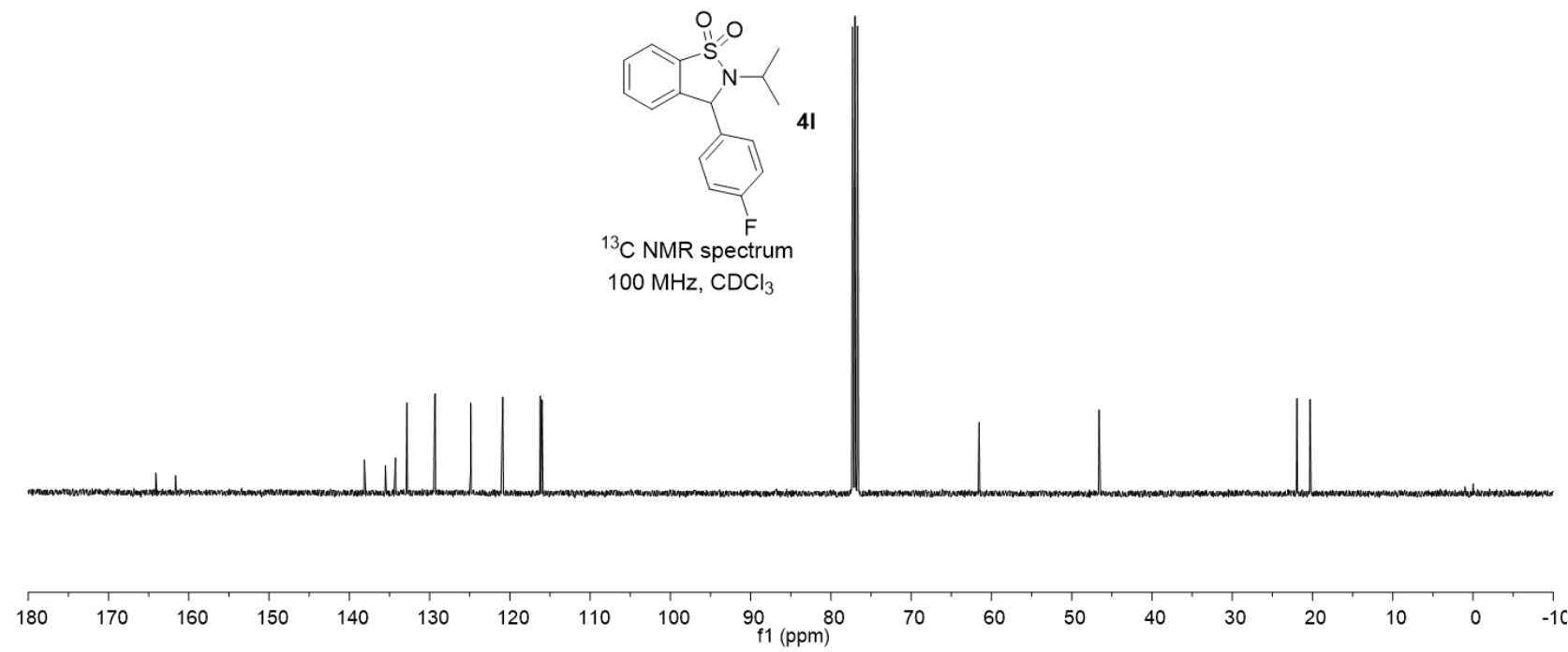
=ப

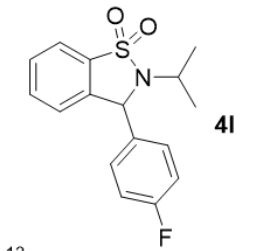

${ }^{13} \mathrm{C}$ NMR spectrum

$100 \mathrm{MHz} \mathrm{CDCl}_{3}$
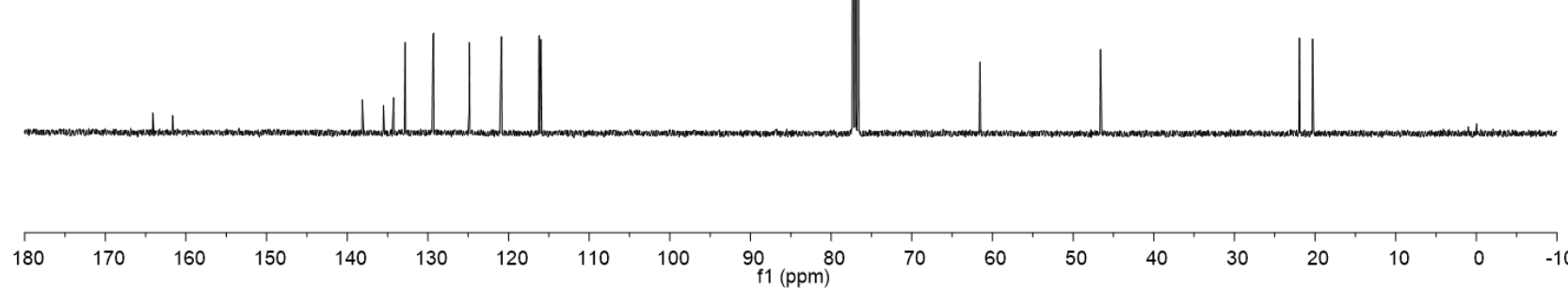

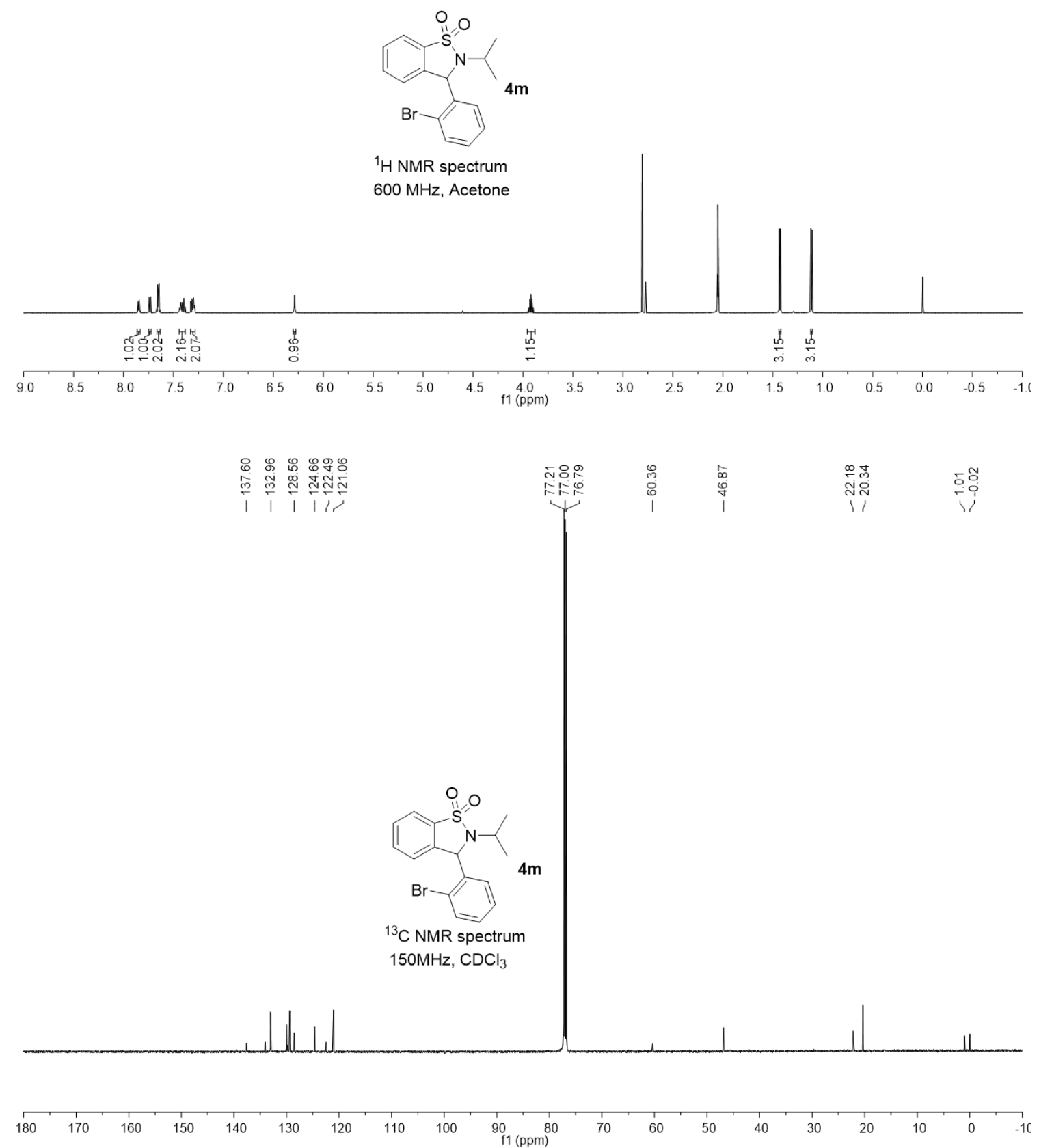

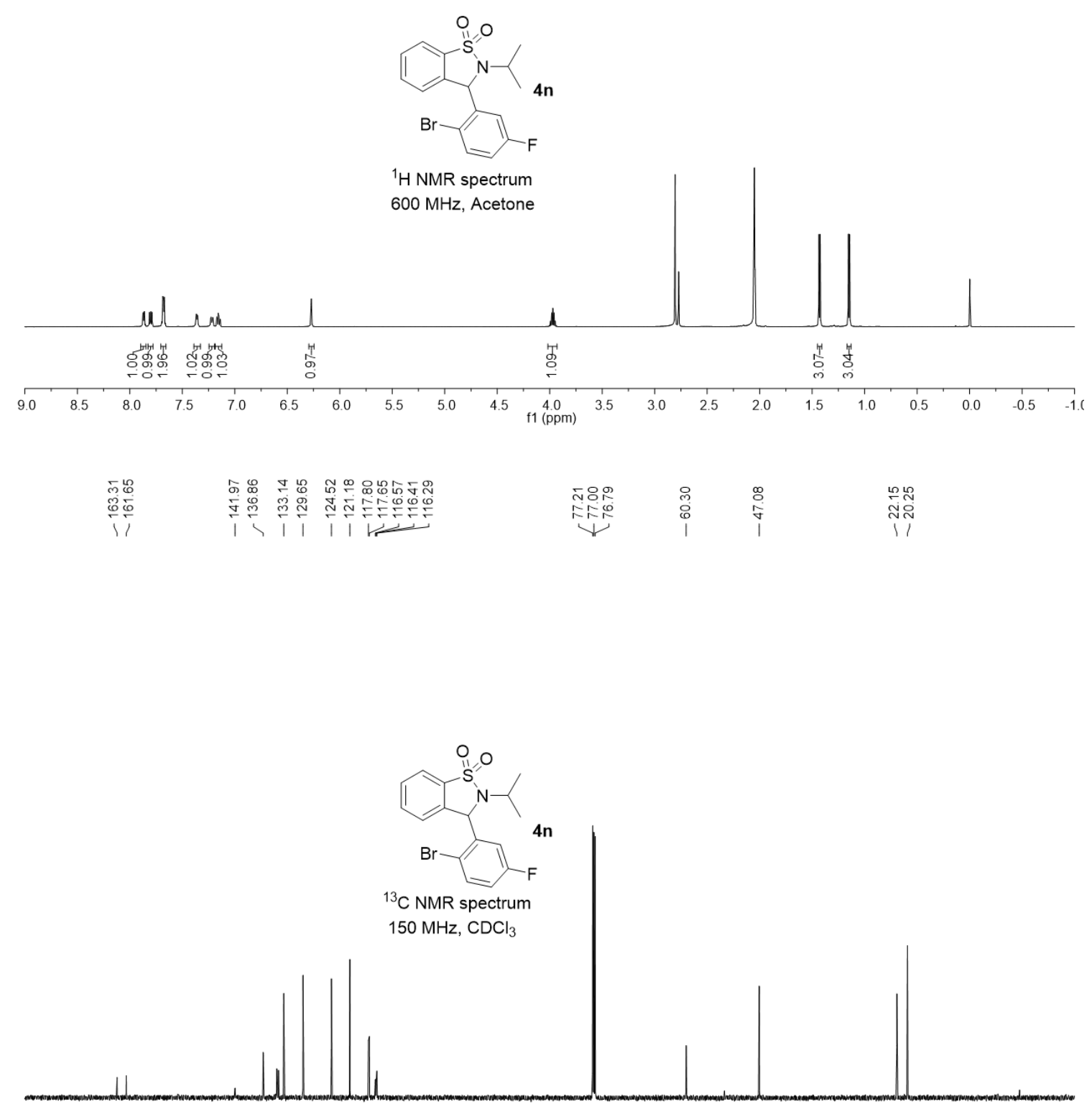

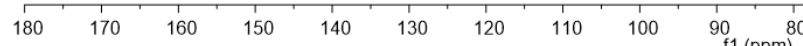




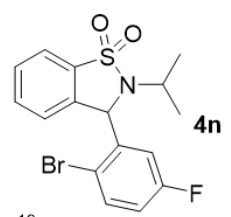

${ }^{19} \mathrm{~F}$ NMR spectrum

$375 \mathrm{MHz}, \mathrm{CDCl}_{3}$
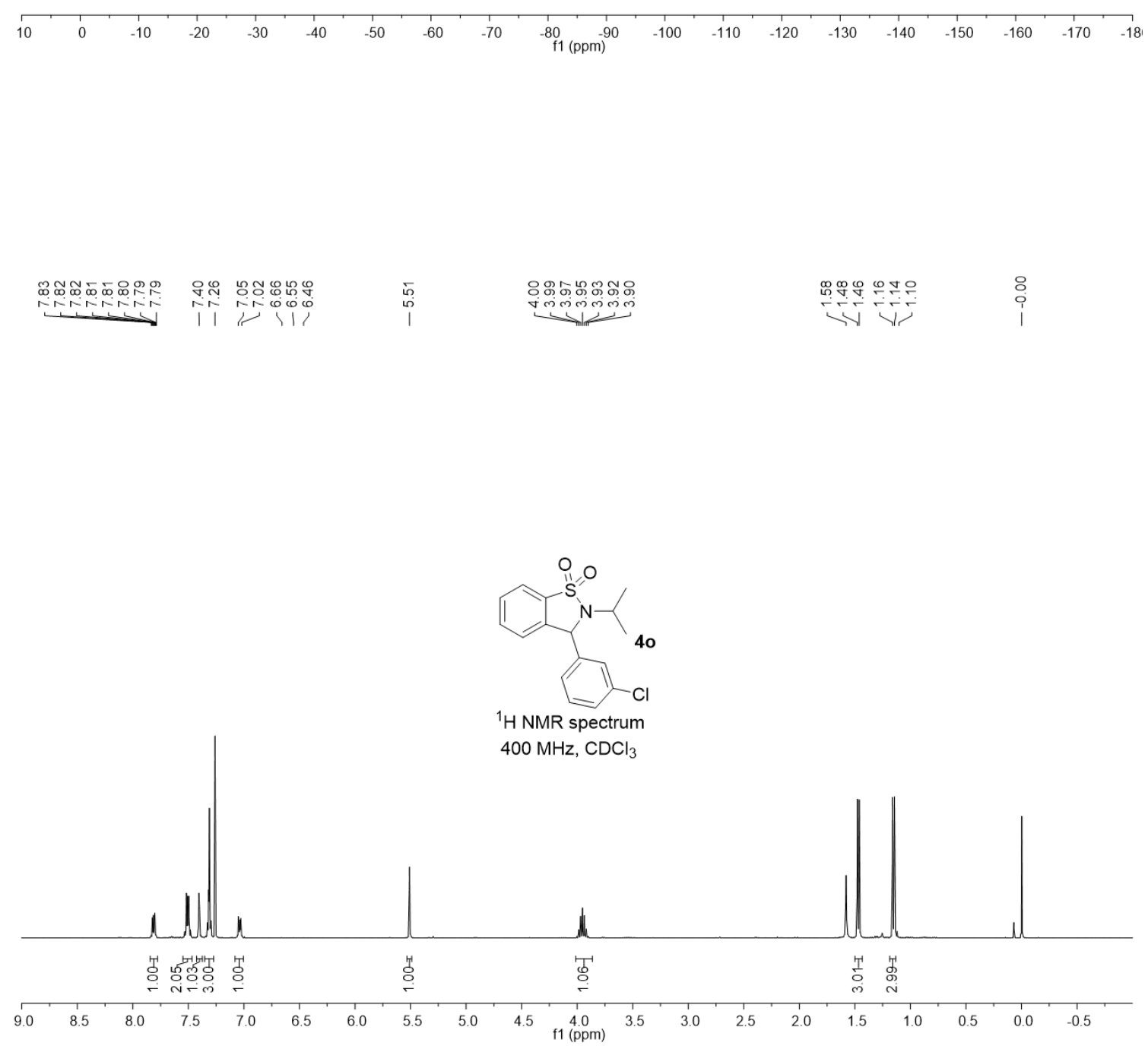


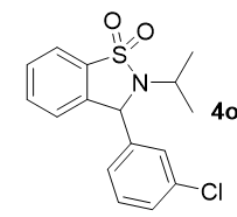

${ }^{13} \mathrm{C}$ NMR spectrum $100 \mathrm{MHz}, \mathrm{CDCl}_{3}$
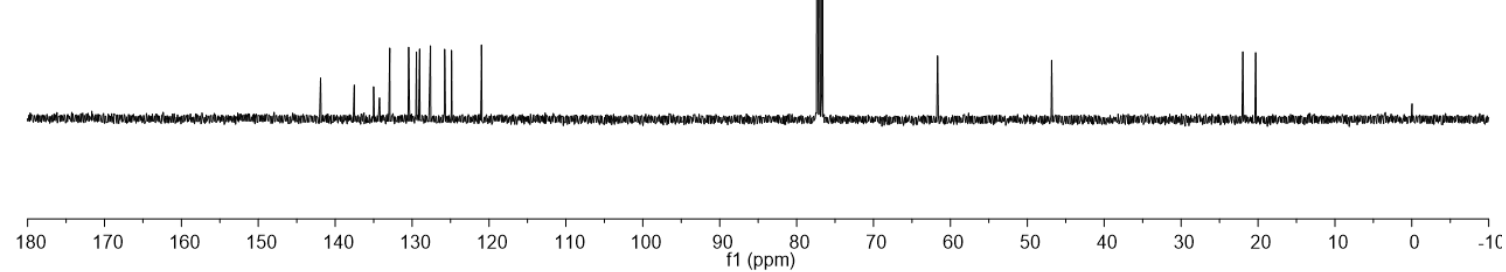

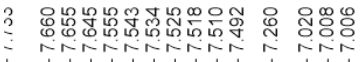

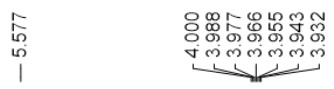

نํำ

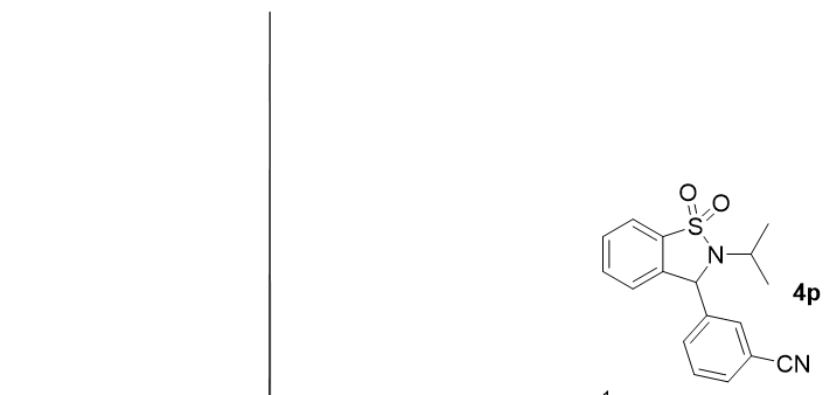

${ }^{1} \mathrm{H}$ NMR spectrum

$600 \mathrm{MHz} \mathrm{CDCl}_{3}$

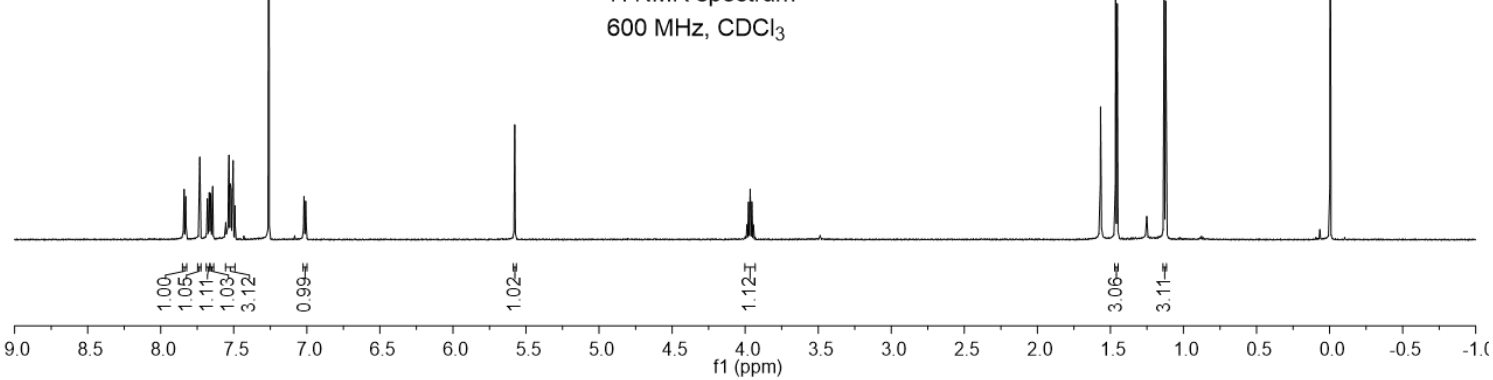




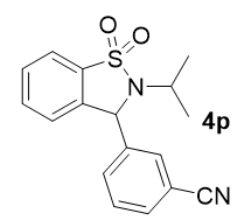

${ }^{13} \mathrm{C}$ NMR spectrum $150 \mathrm{MHz}, \mathrm{CDCl}_{3}$

\begin{tabular}{|c|c|c|c|c|c|c|c|c|c|c|c|c|c|c|c|c|}
\hline 180 & 170 & 160 & 150 & 140 & 130 & 120 & 110 & 100 & $\underset{\mathrm{f} 1(\mathrm{ppm})}{80}$ & 70 & 60 & 50 & 40 & 30 & 20 & 10 \\
\hline & & 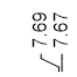 & in & \begin{tabular}{cc}
0 \\
\multirow{2}{*}{} & 9 \\
0 & 0 \\
1 & 1
\end{tabular} & & & 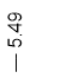 & & 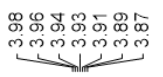 & & & $\underset{\sim}{\sim}$ & $\underbrace{8006}$ & $\frac{\substack{ㅇ \\
ㄷ}}{\check{r}}$ & & $\begin{array}{l}8 \\
0 \\
i \\
1\end{array}$ \\
\hline
\end{tabular}

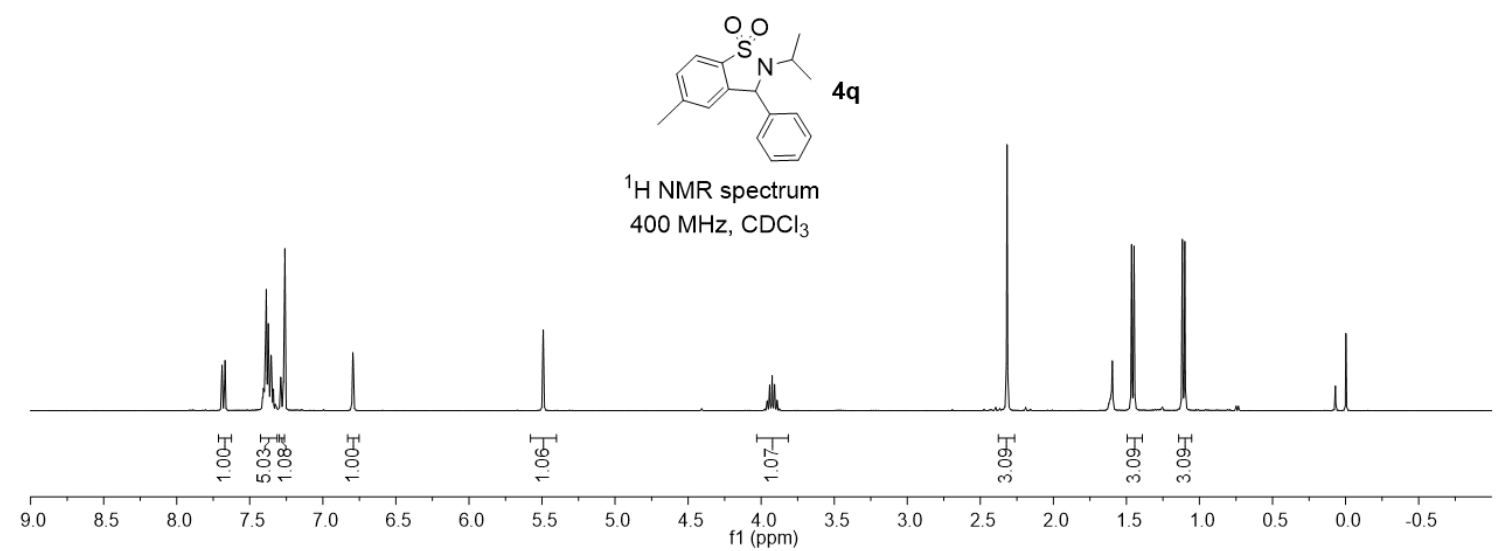




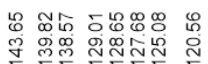

| । पा ।
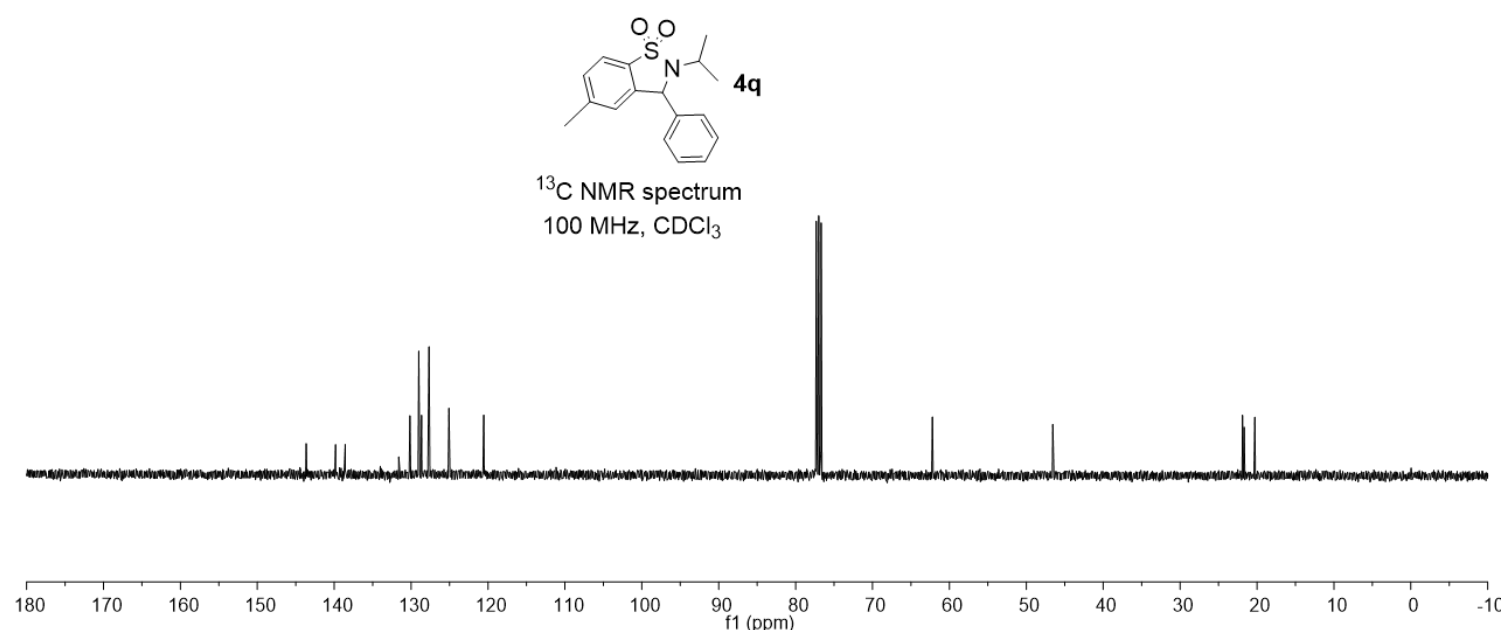

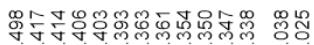

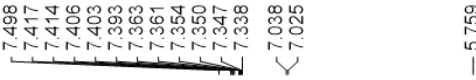

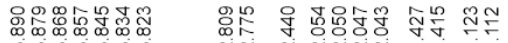

¿̄

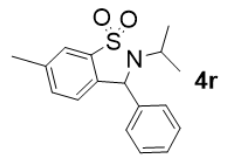

${ }^{1} \mathrm{H}$ NMR spectrum

$600 \mathrm{MHz}$, Acetone

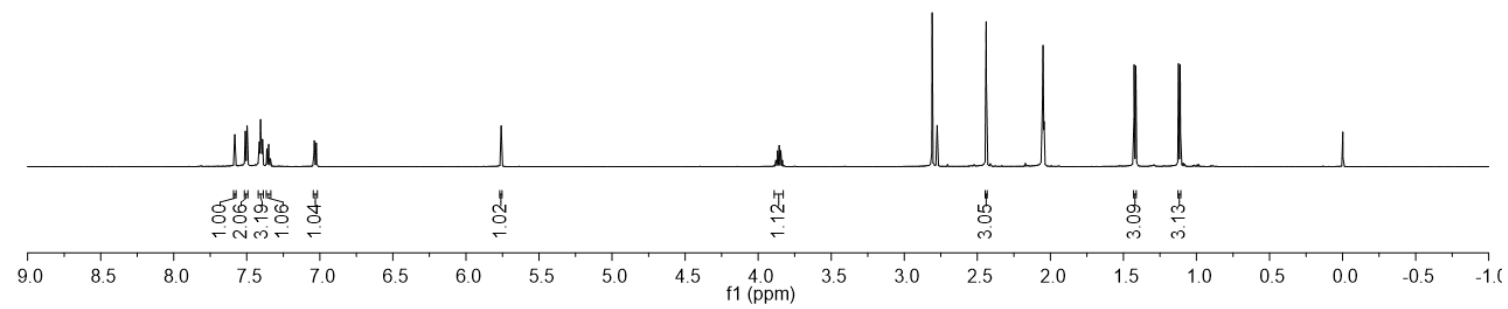



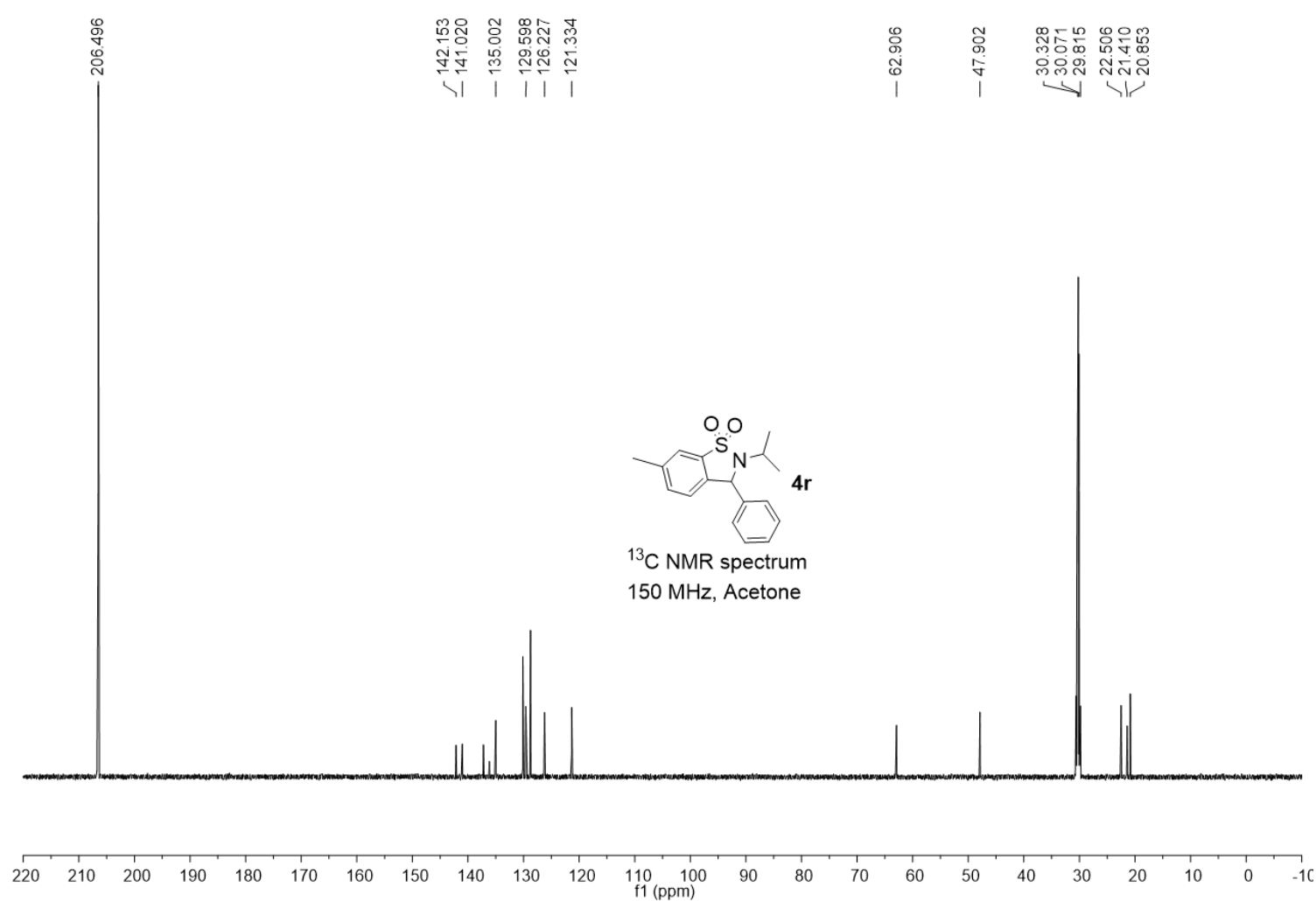

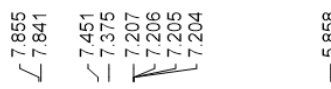
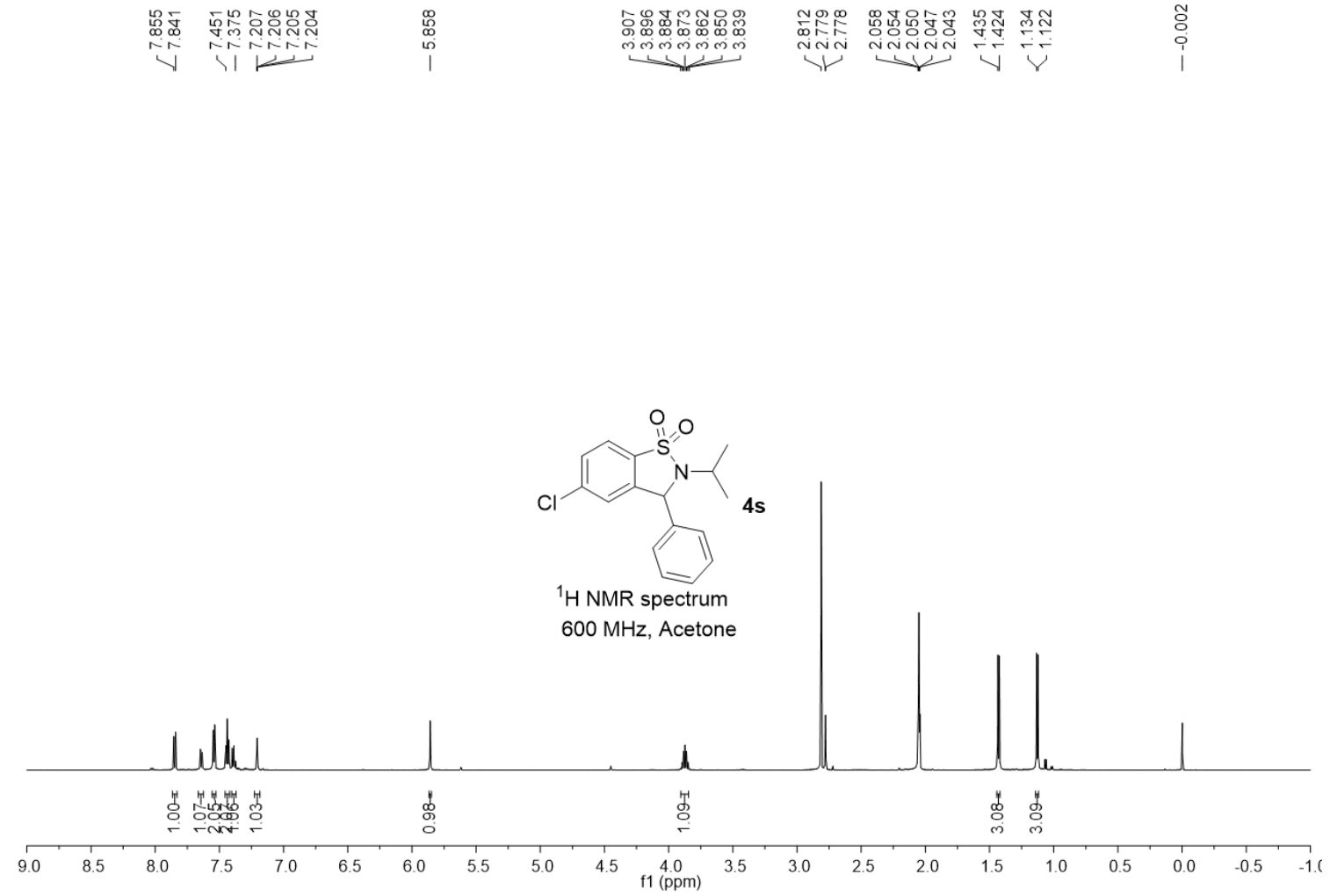


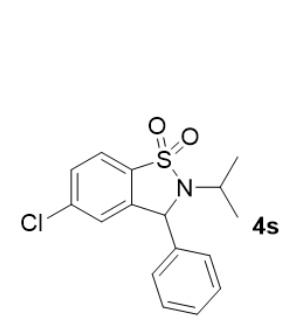

${ }^{13} \mathrm{C}$ NMR spectrum

$150 \mathrm{MHz}, \mathrm{CDCl}_{3}$
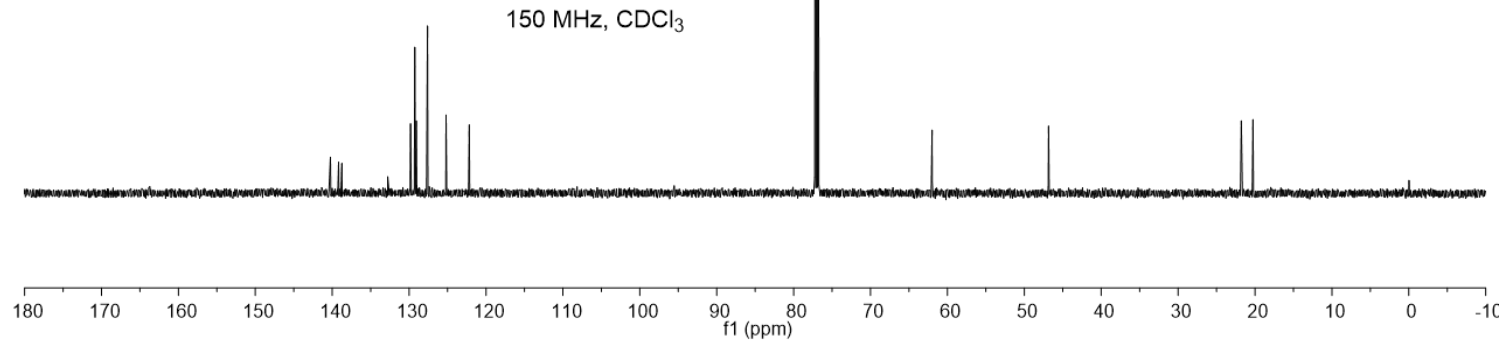

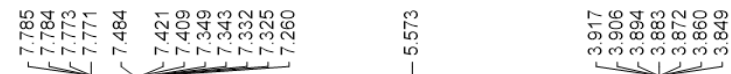

نั

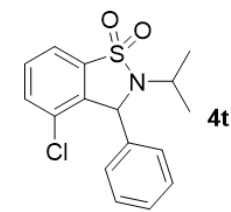

${ }^{1} \mathrm{H}$ NMR spectrum $600 \mathrm{MHz}, \mathrm{CDCl}_{3}$

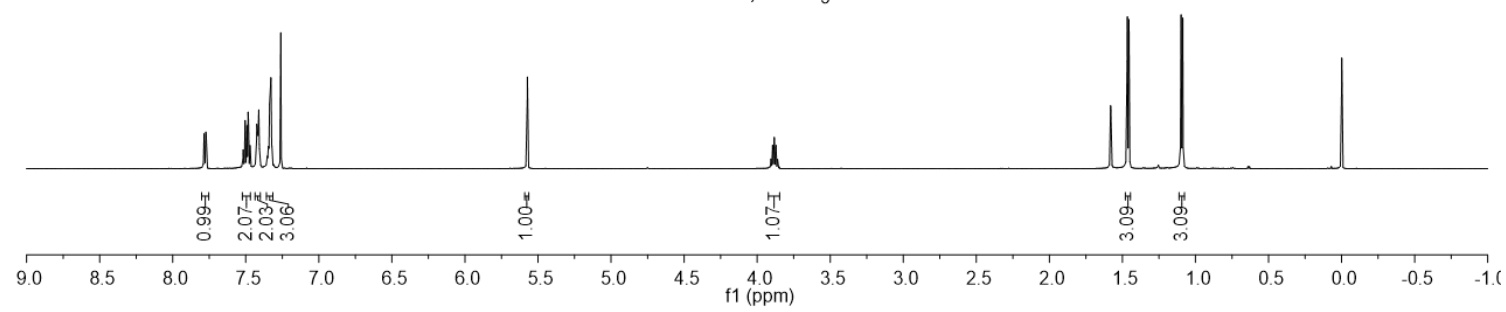




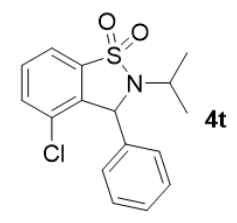

${ }^{13} \mathrm{C}$ NMR spectrum $100 \mathrm{MHz}, \mathrm{CDCl}_{3}$
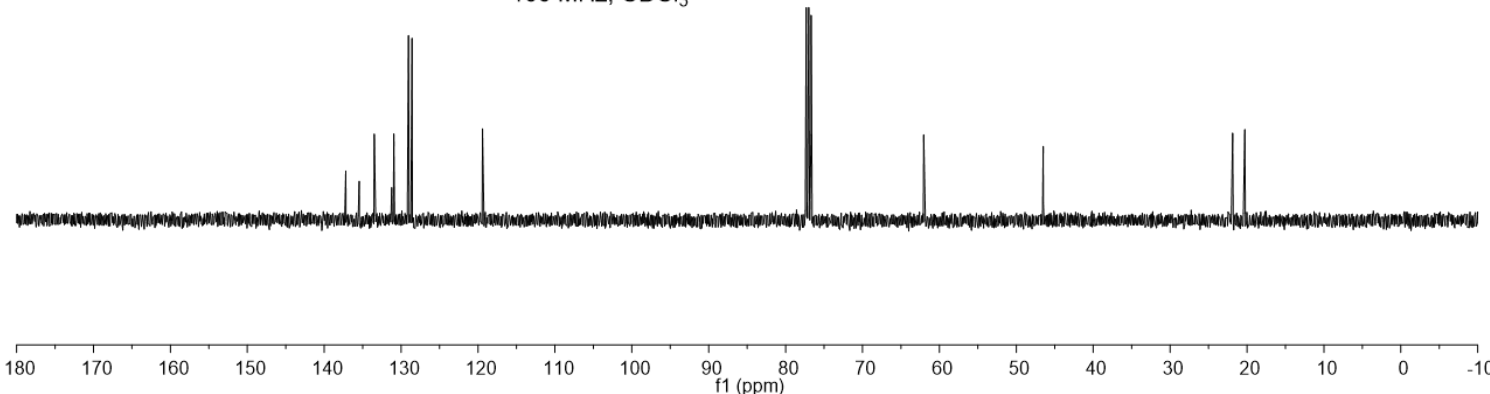

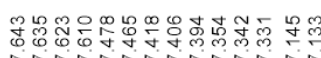

nNanNañNan

4u

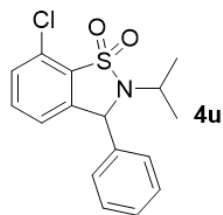

${ }^{13} \mathrm{H}$ NMR spectrum $600 \mathrm{MHz}$, DMSO
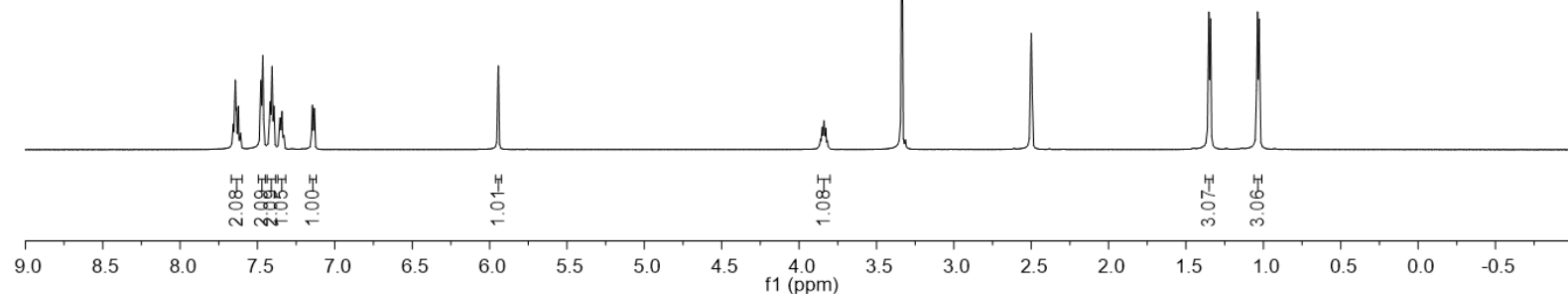


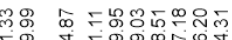

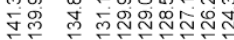
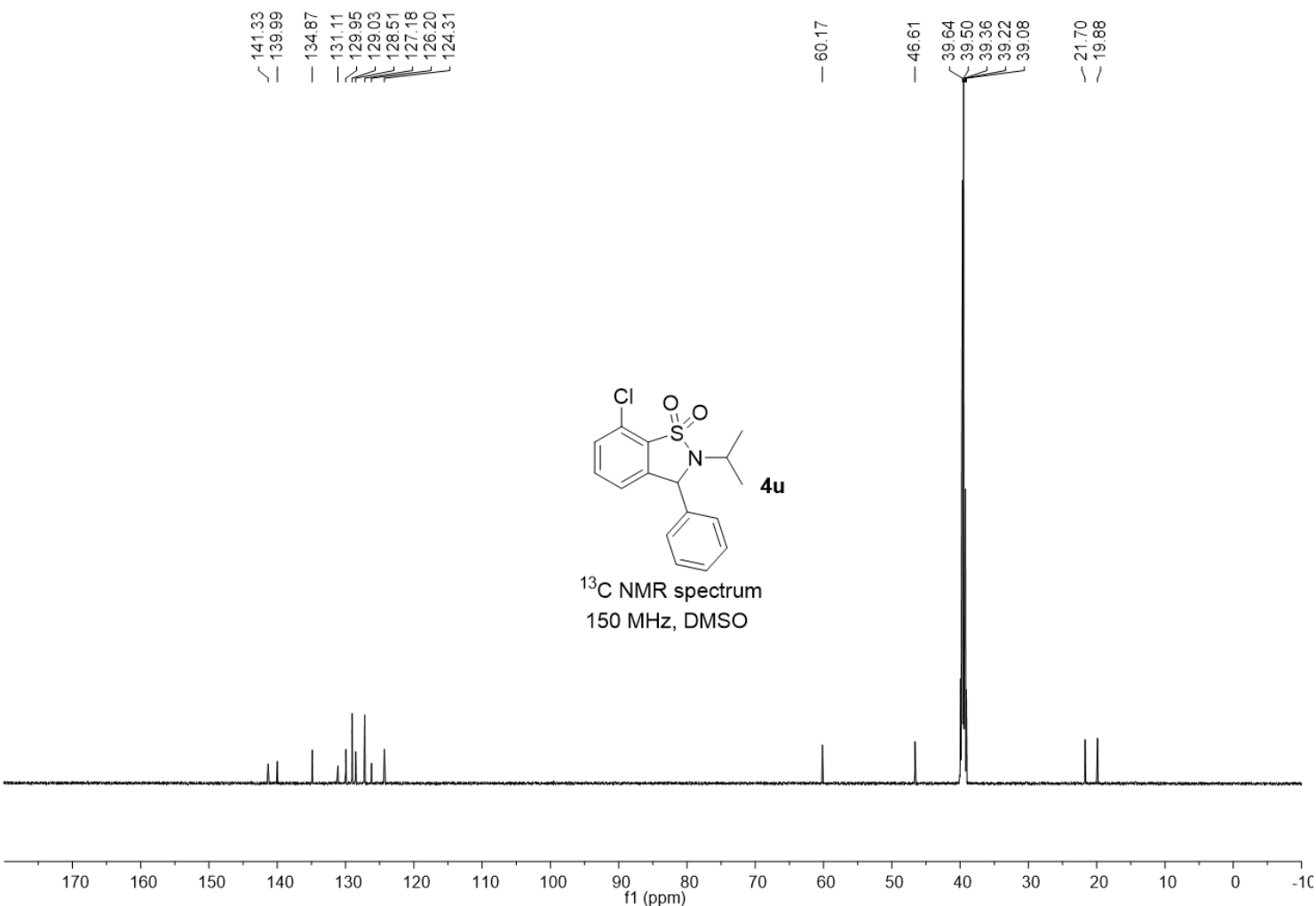